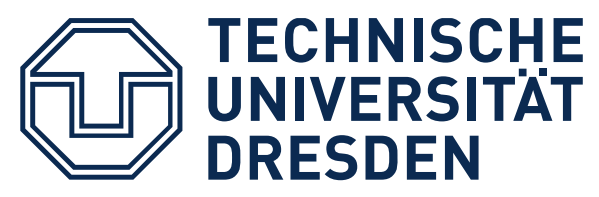

Fakultät Verkehrswissenschaften „Friedrich List“ Institut für Automobiltechnik Dresden Professur für Fahrzeugmechatronik

\title{
Simulationsbasierte Systemauslegung von Elektrobuslinien
}

Dipl.-Ing. Martin Ufert

geboren am 19. Februar 1988 in Dresden

\section{Dissertation}

zur Erlangung des akademischen Grades

Doktoringenieur (Dr.-Ing.)

Erstgutachter

Prof. Dr.-Ing. Bernard Bäker

Zweitgutachter

Prof. Dr.-Ing. Arnd Stephan

eingereicht am: 02.12.2019

verteidigt am: $\quad 07.07 .2020$ 



\section{Danksagung}

Die vorliegende Arbeit entstand während meiner Tätigkeit als wissenschaftlicher Mitarbeiter an der Professur für Fahrzeugmechatronik der Technischen Universität Dresden. Mein Dank gilt Prof. Dr.-Ing. Bernard Bäker, welcher den Rahmen zur Bearbeitung des Forschungsprojektes gegeben und die Betreuung meiner Dissertation übernommen hat. Des Weiteren danke ich Prof. Dr.-Ing. Arnd Stephan für die Übernahme des Zweitgutachtens.

Weiterhin danke ich meinen ehemaligen Kolleginnen und Kollegen der Professur für Fahrzeugmechatronik für das stets kollegiale und freundschaftliche Miteinander. Besonders möchte ich Alexander Bunzel für die sehr gute Zusammenarbeit im Forschungsprojekt und Lutz Morawietz für die stets kritischen, jedoch immer konstruktiven und fachlich anspruchsvollen Diskussionen danken. Für die Unterstützung während der Phase des Korrekturlesens gilt mein Dank Georg Beierlein, Lutz Morawietz, Christoph Pache und Enrico Brandes sowie meiner Familie.

Zusätzlich bedanke ich mich bei der Dresdner Verkehrsbetriebe AG, die im Forschungsprojekt als Projektpartner fungiert hat. Stellvertretend für das ganze Unternehmen danke ich Robert Roch für die Unterstützung und vielen Diskussionsrunden bei der Einordnung zahlreicher wissenschaftlicher Ideen und Ergebnisse in den betrieblichen Alltag eines Verkehrsunternehmens.

Ich bedanke mich außerdem bei allen Studenten, die über Studien- und Diplomarbeiten sowie als studentische Hilfskräfte zum Gelingen dieser Arbeit beigetragen haben.

Besonderer Dank gilt meiner Familie, die mich in meinem Werdegang stets unterstützt hat und ohne die ich nicht zu diesem Punkt gelangt wäre. Abschließend gilt mein größter Dank meiner Frau Sophie für das Verständnis sowie die nahezu grenzenlose Geduld und Unterstützung, welche notwendig war, um diese Arbeit anzufertigen. 



\section{Kurzfassung}

Die anhaltende Diskussion um die Einhaltung gesetzlicher Vorgaben und Grenzwerte von Emissionen in Großstädten veranlassen kommunale Verkehrsunternehmen zunehmend zur Umstellung ihrer Flotten von konventionellen Dieselbussen hin zu Elektrobussen. Aufgrund des geringeren Energieinhalts, der begrenzten Lebensdauer und der oftmals zeitaufwändigen Nachladung der Traktionsbatterien erfordert die Einführung von Elektrobussen eine komplexe Systemauslegung, welche das Zusammenspiel aus Fahrzeug, Betrieb und Ladeinfrastruktur berücksichtigt.

Der Fokus bisheriger Forschungsarbeiten zu diesem Thema liegt mehrheitlich auf einem grundlegenden Technologievergleich sowie der Berechnung der Total-Cost-ofOwnership. Dabei wird für die Systemauslegung oftmals ein heuristisches Vorgehen mit empirischen Energieverbrauchswerten auf Basis linearisierter Modellansätze angewendet. Besonders die Traktionsbatterien der Fahrzeuge weisen jedoch ein stark nichtlineares Betriebsverhalten auf.

In der vorliegenden Arbeit wird eine Simulationsumgebung für einzelne Elektrobuslinien entwickelt. Diese ermöglicht es, das Betriebsverhalten unterschiedlicher Systemkonfigurationen aus Fahrzeug, Traktionsbatterie und Nachladestrategien zu berechnen und zu vergleichen. Kern der Simulation ist ein zweistufiges Batteriemodell. Eine innere Schleife berechnet auf Basis von Mikrozeitschritten die elektro-thermische Belastung der Batterie, auf deren Basis in der äußeren Schleife die resultierende Schädigung und Lebensdauererwartung ermittelt werden. Zusätzlich wird eine Optimierungsfunktion mit dem Ziel implementiert, die elektrobusspezifischen Systemkosten mittels einer wirtschaftlichen Systemauslegung und einer angepassten Nachladestrategie zu reduzieren. Dadurch wird die Ermittlung eines wirtschaftlich optimalen Systems ermöglicht, wobei die Minimierung der Systemkosten als Optimierungsziel dient. Der Modellansatz basiert auf einer Differenzkostenanalyse.

In einem demonstrierten Anwendungsbeispiel beträgt das maximale Optimierungspotential mehr als $13 \%$, während das mittlere Potential mehr als $5 \%$ beträgt. Aus den absoluten Ergebnissen der Optimierungsrechnungen werden Sensitivitäten einzelner Kostenparameter abgeleitet. Daraus geht hervor, dass Energiekosten aktuell den größten Kostenbestandteil des Systems darstellen. Gleichzeitig weisen sie auch die höchste Sensitivität auf und bieten somit ein hohes Potential zur Kostenreduzierung von Elektrobussystemen. 



\section{Abstract}

The ongoing discussion about compliance with legal requirements and emission limits in major cities is increasingly encouraging public transport companies to convert their fleets from conventional diesel buses to electric buses. Due to the lower energy content, the limited lifetime and the often time-consuming process of recharging of the traction batteries, the introduction of electric buses requires a complex system design that takes into account the interaction of vehicle, operation and recharging infrastructure.

The focus of previous work in this field has mainly focused on a fundamental technology comparison and the calculation of the total cost of ownership. For the system design, a heuristic procedure with empirical energy consumption values based on linearized model approaches is often applied. However, especially the traction batteries of the vehicles show a strongly non-linear operating behaviour.

In this thesis a simulation framework for single electric bus lines is developed. This allows to calculate and compare the operating behaviour of different system configurations of vehicle, traction battery and recharging strategies. The core of the simulation is a two-stage battery model. An inner loop calculates the electro-thermal load on the battery on the basis of micro time steps. The resulting damage and life expectancy are then determined in the outer loop. In addition, an optimization function is implemented with the aim of reducing the electro-bus-specific costs by means of an efficient system design and an adapted recharging strategy. This enables the determination of an economically optimal system, whereby the minimization of system costs serves as the optimization goal. The model approach is based on a differential cost analysis.

In a demonstrated application example, the maximum optimization potential is higher than $13 \%$, while the average potential is higher than $5 \%$. Sensitivities of individual cost parameters are derived from the absolute results of the optimization calculations. These show that energy costs currently represent the largest cost component of the system. At the same time, they also show the highest sensitivity and thus offer a high potential for cost reduction of electrical bus systems. 



\section{Inhalt}

1 Einleitung .............................................................................................................. 1

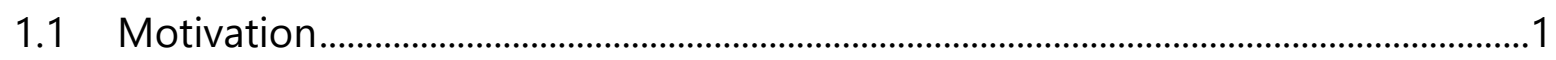

1.2 Ziele der Arbeit.............................................................................................................

1.3 Aufbau der Arbeit............................................................................................................

2 Das System Elektrobus - Bestandteile und Auslegungsmethodik ...................... 7

2.1 Anforderungen, Systembestandteile und Nachladung ..............................................7

2.2 Stand der Forschung ...................................................................................................... 11

2.3 Ableitung des Forschungsbedarfs............................................................................. 16

3 Konzept zur simulationsbasierten Systemauslegung einer Elektrobuslinie .. 19

3.1 Herleitung wesentlicher Freiheitsgrade in der Systemauslegung ........................ 19

3.2 Entwicklung des Simulationskerns der Systemsimulation .......................................22

3.3 Definition wesentlicher Randbedingungen zur Systemsimulation ......................24

3.3.1 Traktionsbatterie ………………………………………………………………... 25

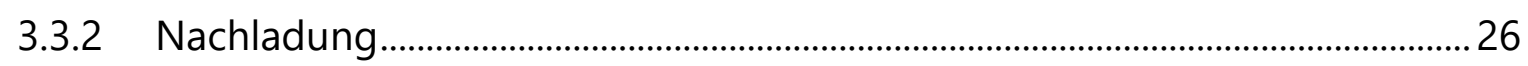

3.4 Konzept einer mehrdimensionalen Optimierung mittels

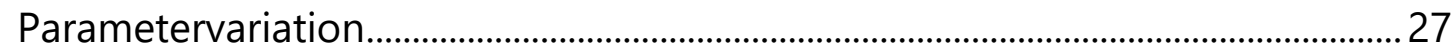

3.5 Entwicklung des Frameworks zur Systemsimulation und Optimierung ..............31

4 Modellbildung und Modellvalidierung ................................................................35

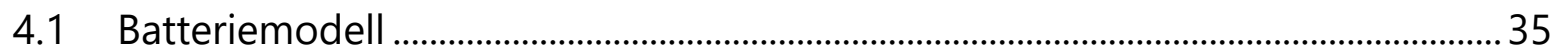

4.1.1 Definition der Modellierungs- und Betrachtungsebenen ..................................35

4.1.2 Modellbildung, Funktionsprinzip und Kenngrößen von Li-lonen Zellen....36

4.1.3 Elektrisches Modell......................................................................................................4

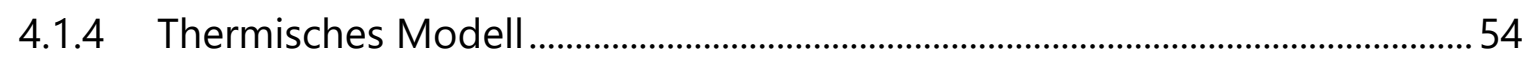

4.1.5 Alterungsmodell ............................................................................................................. 67

4.2 Modell der Nachladepunkte............................................................................................. 86

4.3 Kostenmodell ................................................................................................................ 88 
5 Systemsimulation und Optimierung ……........................................................95

5.1 Entwicklung verschiedener Einsatzszenarien .........................................................95

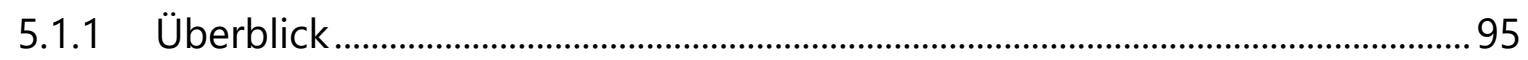

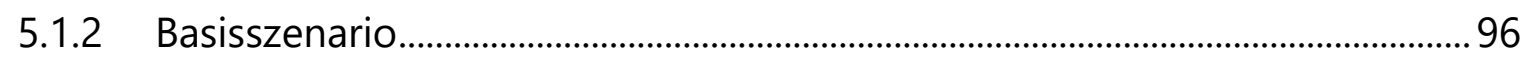

5.1.3 Betriebs- und Ruhetage ......................................................................................

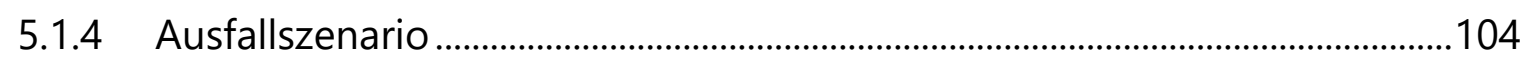

5.1.5 Temperaturverteilung..........................................................................................107

5.1.6 Fahrzeugbetrieb mit Batterieheizung ……………………………………………....111

5.2 Detailuntersuchungen zur Alterung der Traktionsbatterie ...................................113

5.2.1 Sensitivitätsanalyse der Makrozeitschrittweite ..................................................113

5.2.2 Sensitivitätsanalyse der Schädigungsfaktoren ...................................................116

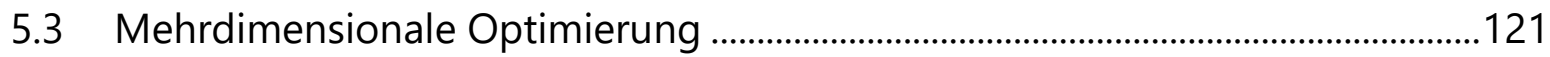

5.3.1 Optimierung auf Grundlage des Basisszenarios .................................................121

5.3.2 Optimierung mit verteilten Temperaturen, Ruhetagen und Ausfallszenarien .......................................................................................................128

5.3.3 Vergleich und Bewertung der Optimierungsrechnungen ...............................131

5.4 Sensitivitätsanalyse betrieblicher und ökonomischer Parameter ........................135

5.4.1 Einfluss des Fahrplantaktes auf die optimale Systemkonfiguration............135

5.4.2 Sensitivitätsanalyse der einzelnen Kostenbestandteile....................................136

6 Zusammenfassung, Fazit und Ausblick ........................................................... 139

6.1 Zusammenfassung und Fazit der Arbeit ...................................................................139

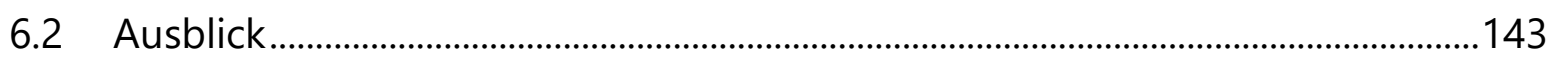

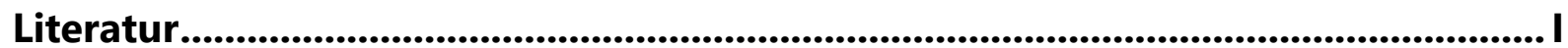

Abkürzungs- und Symbolverzeichnis ........................................................................ VII

Abbildungsverzeichnis ...................................................................................................XI

Tabellenverzeichnis..................................................................................... XV

Anhang ........................................................................................................................... XVII 


\section{$1 \quad$ Einleitung}

\subsection{Motivation}

Getrieben durch die anhaltende Diskussion um saubere Luft in deutschen Innenstädten und dem damit verbundenen Trend hin zu nachhaltigen Verkehrskonzepten forcieren derzeit viele kommunale Verkehrsunternehmen die Umstellung ihrer Busflotten von Dieselbussen auf Elektrobusse. Diese ist in vielen Städten ein elementarer Baustein der Maßnahmenpakete zur Reduzierung von Emissionen und Einhaltung gesetzlicher Grenzwerte. In den vergangenen Jahren wurden im Rahmen verschiedener Förderprogramme viele Pilotprojekte gestartet und abgeschlossen, beispielsweise in Berlin, Dresden, Hamburg oder Braunschweig (BMVI, 2016). In Dresden wurde z.B. ein zwölfMeter Elektrobus mit einem konduktiven Ladesystem im Rahmen des Projekts "Elektrobuslinie 79" ausgiebig getestet (Bunzel et al., 2016). Ziel aller Projekte war, die unterschiedlichen am Markt verfügbaren Systeme hinsichtlich Einsatzreife zu testen sowie erste Erfahrungen im Umgang mit Elektrobussen und deren Einsatz im regulären Fahrgastbetrieb zu sammeln. Eine Übersicht der Pilotprojekte in Deutschland und Europa ist in (UITP, 2017) gegeben. In vielen dieser Projekte wurden hauptsächlich Fahrzeuge beschafft und in Betrieb genommen, welche auf speziell ausgewählten Strecken zum Einsatz kamen - oftmals mit geringer täglicher Fahrleistung, um die bestehende Reichweitenproblematik zu umgehen. Nur in wenigen Projekten war die Anzahl der beschafften Fahrzeuge ausreichend, um beispielsweise eine ganze Linie mit Elektrobussen zu betreiben.

In den kommenden Jahren soll nun der nächste Schritt hin zur Umstellung von Busflotten auf Elektromobilität vollzogen werden. Dabei planen viele Verkehrsbetriebe die Beschaffung einer größeren Anzahl an Fahrzeugen, mit denen dann beispielsweise ganze Linien vollständig mit Elektrobussen betrieben werden können. Für eine möglichst hohe Wahrnehmbarkeit in der Öffentlichkeit besteht häufig der Wunsch, so genannte Volumenlinien zu wählen. Dabei handelt es sich um Linien, welche ein hohes Fahrgastaufkommen und oftmals auch eine hohe tägliche Fahrleistung aufweisen. Damit ergeben sich jedoch entsprechende Anforderungen an die Gefäßgröße und die Reichweite der Fahrzeuge.

In Abbildung 1-1 ist eine Roadmap für die Einführung von Elektrobussen gegeben. Nach erfolgreichem Abschluss der (o.g.) Pilotprojekte wird nun eine Teilumstellung der Flotte angestrebt. Eine sofortige vollständige Flottenumstellung ist unter den gegebenen Bedingungen (hohe Investkosten, Marktverfügbarkeit der Fahrzeuge, Aufbau und Ausbau der Ladeinfrastruktur) in Deutschland zurzeit nur schwer realisierbar. Trotz 
teilweise hoher Subventionen stehen die Verkehrsunternehmen unter starkem wirtschaftlichem Druck, sodass für viele Kommunen nur eine schrittweise Umstellung in Frage kommt. Demnach folgt auf ein Pilotprojekt eine Teilumstellung der Flotte, was in vielen Anwendungsfällen einer Linienumstellung entspricht. Die abgebildete Roadmap zeigt jedoch eine idealisierte Vorgehensweise zur Einführung von Elektrobussen. In vielen Städten folgte nach der Planungsphase sowie dem Pilotbetrieb eine kritische Evaluierung der Ergebnisse, die zu einer erneuten Planungsphase mit angepassten Eingangsparametern und Randbedingungen geführt hat. Dies hatte beispielsweise zur Folge, dass eine deutsche Großstadt für die Teilumstellung der Flotte eine andere Ladetechnologie verwendet als im Pilotprojekt getestet wurde (Neuendorff, 2018). Als eine weitere mögliche Ausgestaltung der Roadmap ist vermehrt zu beobachten, dass Verkehrsunternehmen die Pilotbetriebsphase überspringen und direkt eine Teilumstellung vollziehen, wobei die Erfahrungen aus diversen Pilotprojekten anderer Verkehrsunternehmen herangezogen werden.

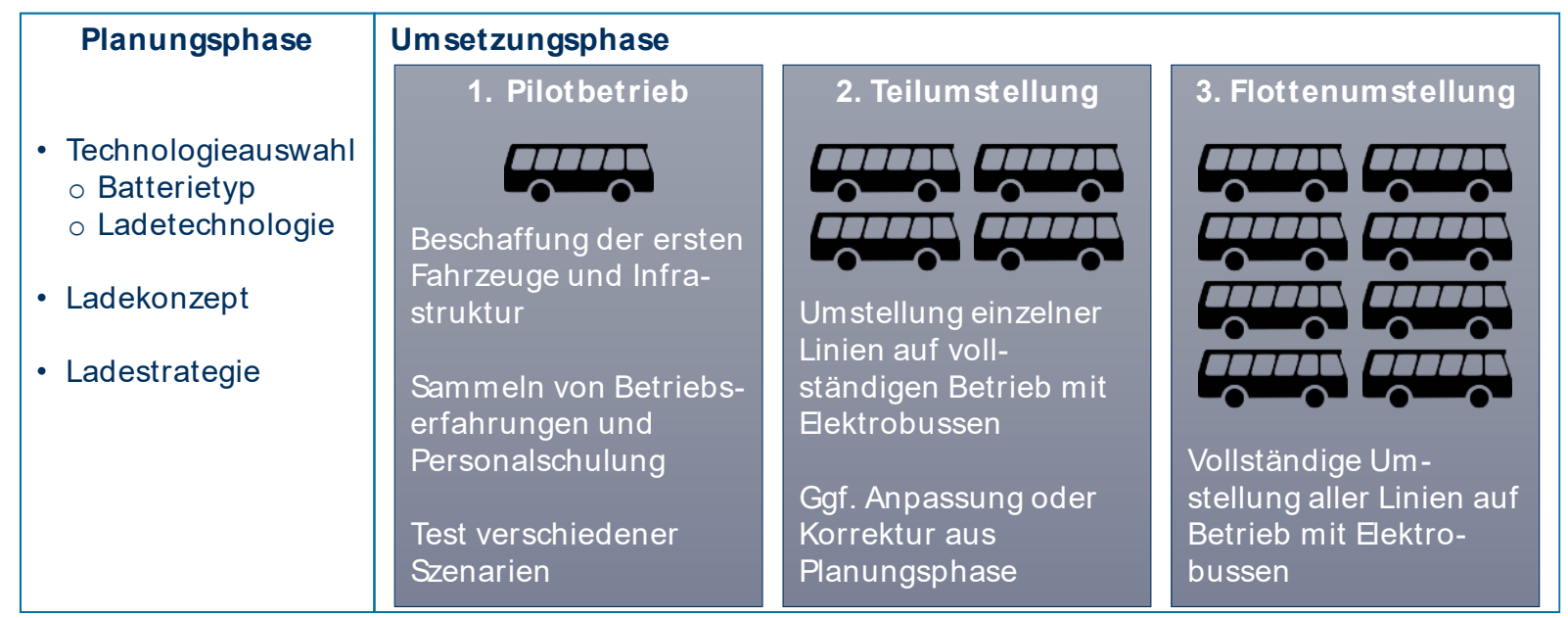

Abbildung 1-1: Roadmap zur Einführung von Elektrobussen, nach (Corneille, 2018)

Unabhängig davon, welchen der aufgezeigten Wege ein Unternehmen wählt, ist in vielen Fällen der nächste nun anstehende Schritt auf dem Weg zur Elektromobilität im ÖPNV eine Teilumstellung der Flotte. Hier bietet es sich an, die Elektrobusse nicht im Mischbetrieb mit Dieselbussen auf einer Linie zu betreiben, sondern "reine" Elektrobuslinien aufzubauen. Dadurch können weitere und detailliertere Erfahrungen in den Bereichen Fahrzeugzuverlässigkeit und -verfügbarkeit sowie Pünktlichkeit im Betrieb gewonnen werden, welche dann auf dem Weg zur letzten Realisierungsphase, einer vollständigen Flottenumstellung, von entscheidender Bedeutung sind.

Eines der wichtigsten Kriterien bei der Wahl einer geeigneten Linie für den Betrieb von Elektrobussen ist die realisierbare Reichweite der Fahrzeuge. Die derzeit am Markt 
verfügbaren Elektrobusse weisen jedoch sehr unterschiedliche Reichweiten auf. Die Herstellerangaben gehen dabei von ca. $150 \mathrm{~km}$ für einen Solobus $(12 \mathrm{~m})$ bis hin zu über $300 \mathrm{~km}$ für einen Gelenkbus (18 m) (Bloomberg, 2018). Je nach Wahl der Linie kann diese Reichweite jedoch zu gering sein, um einen zuverlässigen Betrieb unter allen Umständen zu gewährleisten. In solchen Fällen ist die Nachladung des Fahrzeugenergiespeichers während des Betriebs notwendig, um die tägliche Fahrleistung der Linie erbringen zu können. Die investiven Kosten (Anfangsaufwendungen) von Elektrobussen liegen aktuell noch deutlich über denen von Dieselbussen (Bloomberg, 2018). Als wesentliche Gründe dafür werden in der Quelle die hohen Kosten der Batterie ${ }^{1}$ sowie die geringe Stückzahl gefertigter Fahrzeuge genannt. Zusätzlich ist, unabhängig vom Ladekonzept und der Ladetechnologie, eine Investition in die Ladeinfrastruktur erforderlich. In Summe ergeben sich damit Zusatzkosten für die Einführung von Elektrobussen im Vergleich zu Dieselbussen. Deren Höhe ist sehr stark von der jeweils konkreten Umsetzung abhängig und kann grundsätzlich nicht verallgemeinert werden.

Als Stand der Technik werden in Elektrobussen heute nahezu ausschließlich Traktionsbatterien auf Li-Ionen Basis eingesetzt. Wie im weiteren Verlauf der Arbeit noch gezeigt werden wird, unterliegen diese einem ständigen Alterungsprozess, wodurch sich deren Leistungsfähigkeit kontinuierlich reduziert und diese letztendlich einen Punkt erreicht, wo sie den Anforderungen in einem Elektrobus nicht mehr genügt. Zum gegenwärtigen Zeitpunkt ist nicht absehbar, ob die Batterien bereits innerhalb des Fahrzeuglebenszyklus ersetzt werden müssen. Für die Betrachtung der Wirtschaftlichkeit sind neben den anfänglich investiven Kosten somit auch die nachfolgenden Kosten, beispielsweise für einen Ersatz der Traktionsbatterien, zu berücksichtigen.

\subsection{Ziele der Arbeit}

Mit der vorliegenden Arbeit soll ein Beitrag zur Reduzierung der elektrobusspezifischen Zusatzkosten geleistet werden. Öffentlicher Personennahverkehr (ÖPNV) ist in Deutschland nur selten kostendeckend und wird deshalb oftmals von kommunalen Aufgabenträgern subventioniert. ${ }^{2}$ Aus diesem Grund ist ein sensibler Umgang mit den dafür eingesetzten Finanzmitteln erstrebenswert. Zurzeit existieren nahezu keine Fahrzeuge am Markt, welche die Anforderungen an linienbetriebenen ÖPNV, resultierend aus dem jahrzehntelangen Einsatz von Dieselbussen, vollständig abdecken können. Deshalb ist ein eins-zu-eins Ersatz der Fahrzeuge (vollständige Flottenumstellung) nicht

\footnotetext{
${ }^{1}$ Mit dem Begriff Batterie wird im Kontext der Elektromobilität ein wiederaufladbarer, elektrochemischer Speicher beschrieben. Die korrekte Bezeichnung eines solchen Sekundärspeichers (gekennzeichnet durch die Wiederaufladbarkeit) stellt der Begriff Akkumulator dar. In dieser Arbeit wird dennoch der Begriff Batterie synonym für einen Akkumulator verwendet.

${ }^{2}$ Die Dresdner Verkehrsbetriebe AG erreichte im Jahr 2018 einen Kostendeckungsgrad von 80\% (DVB, 2019).
} 
bzw. nur in wenigen Anwendungsfällen möglich. Um dennoch bereits heute Elektrobusse einsetzen zu können, bietet eine linienbezogene Beschaffung einen guten Kompromiss. In der vorliegenden Arbeit soll eine Methodik entwickelt werden, welche unter Beachtung spezifischer Rand- und Einsatzbedingungen eine kosteneffiziente Auslegung und Dimensionierung einzelner Elektrobuslinien ermöglicht.

Eine valide Ermittlung der technischen Machbarkeit ist eine notwendige Bedingung zur Beurteilung der (wirtschaftlichen) Effizienz einer Elektrobuslinie. Im weiteren Verlauf der Arbeit wird gezeigt, dass dafür eine simulationsbasierte Betrachtung erforderlich ist. Dafür sind wiederum valide physikalische Modelle der betroffenen Bereiche erforderlich, um bereits im Planungs- und Dimensionierungsprozess notwendige Entscheidungen für einen effizienten Betrieb treffen zu können. Im Fall der Linienauslegung von Elektrobussen beinhaltet dies u.a. ein Fahrzeugmodell, ein Batteriemodell und ein Modell der eingesetzten Ladeinfrastruktur sowie deren Interaktion untereinander und mit dem geplanten Betriebsprogramm.

In der vorliegenden Arbeit wird eine Simulationsumgebung entwickelt, welche eine Simulation von Elektrobuslinien ermöglicht. Der Fokus liegt neben der validen Modellierung der genannten Teilbereiche auch auf deren Interaktion. Für die simulationsbasierte Auslegung und Dimensionierung werden deshalb in dieser Arbeit die Begriffe Systemauslegung und Systemsimulation verwendet. Zusätzlich wird eine Optimierungsfunktion in die Simulationsumgebung integriert, um für einen konkreten Anwendungsfall ein optimales Systemdesign zu ermitteln.

\subsection{Aufbau der Arbeit}

In Kapitel 2 wird zunächst eine allgemeine Darstellung des Systems Elektrobus vorgenommen sowie die im Rahmen dieser Arbeit zu betrachtenden Systembestandteile abgegrenzt. Anschließend werden verschiedene Konzepte und Technologien zur Nachladung vorgestellt. Der mittlere Teil des Kapitels enthält die Einordnung in den aktuellen Stand der Forschung bezüglich unterschiedlicher Methoden, Herangehensweisen und Granularitäten der Systemauslegung. Es werden die allgemein notwendigen Schritte dafür aufgezeigt und daraus der Forschungsbedarf abgeleitet, welcher die Basis der vorliegenden Arbeit bildet. Das Kapitel schließt mit der Formulierung der Forschungsziele und Forschungsfragen.

In Kapitel 3 wird ein Konzept zur simulationsbasierten Systemauslegung mit einer integrierten Optimierungsfunktion erarbeitet. Es werden zunächst die im Rahmen dieser Arbeit betrachteten Freiheitsgrade bei der Systemauslegung hergeleitet. Der mittlere Teil des Kapitels enthält die Entwicklung des Simulationskerns. Daran anschließend werden notwendige Randbedingungen der Teilsysteme Traktionsbatterie und Nachladung definiert. Im letzten Teil des Kapitels wird die Optimierungsfunktion 
auf Basis einer Parametervariation entwickelt sowie die Einbettung in das Simulationsframework vorgenommen.

In Kapitel 4 werden die einzelnen Bestandteile modelliert, welche für die Systemsimulation erforderlich sind. Diese umfassen im Wesentlichen ein zweistufiges Batteriemodell, bestehend aus einem elektrischen, einem thermischen und einem Alterungsmodell, welche auf unterschiedlichen Zeitebenen arbeiten. Des Weiteren werden ein Modell der Ladeinfrastruktur und ein Kostenmodell entwickelt. Diese bilden die Grundlage für die im folgenden Kapitel durchzuführenden Simulations- und Optimierungsrechnungen.

In Kapitel 5 werden verschiedene technische und betriebliche Einsatzszenarien definiert, welche anschließend mittels der Simulationsumgebung und entwickelten Modelle berechnet und bewertet werden. Dies beinhaltet sowohl die Berechnung einzelner Szenarien als auch diverse Optimierungsrechnungen mit unterschiedlichen Prämissen. Des Weiteren werden Sensitivitäten betrieblicher und ökonomischer Faktoren ermittelt. Aus den Sensitivitäten einzelner Bestandteile der Gesamtkostenfunktion werden Kostensenkungspotentiale für den Einsatz von Elektrobussen aufgezeigt.

Kapitel 6 fasst die Arbeit und deren Ergebnisse zusammen. Die Arbeit schließt mit einem Ausblick auf künftige Forschungsthemen. 



\section{Das System Elektrobus - Bestandteile und Auslegungs- methodik}

In diesem Kapitel werden einleitend die im Rahmen dieser Arbeit relevanten Bestandteile des Systems Elektrobus benannt und beschrieben. Anschließend folgen eine Darstellung des aktuellen Stands der Forschung zum Thema Systemauslegung sowie die Ableitung des Forschungsbedarfs.

\subsection{Anforderungen, Systembestandteile und Nachladung}

Die für die Fahraufgabe notwendige Energie wird bei konventionellen Dieselbussen aus dem Dieselkraftstoff bezogen. Batterie-elektrische Busse besitzen dagegen einen Hochvolt-Traktionsenergiespeicher. Dieser ist die einzige Energiequelle auf dem Fahrzeug, welche zur Bereitstellung der notwendigen Energie für die Fahraufgabe zur Verfügung steht. Der wohl wichtigste Unterschied von Elektrobussen im Vergleich zu Dieselbussen im täglichen Betrieb ist die deutlich limitierte Reichweite. Die in der Batterie speicherbare Energiemenge ist deutlich geringer als bei einem volumen- und gewichtsmäßig vergleichbaren Kraftstofftank. Die folgende Beispielrechnung in Tabelle 2-1 verdeutlicht diese Aussage.

Tabelle 2-1: Überschlägiger Reichweitenvergleich von Dieselbus und Elektrobus ${ }^{3}$

\begin{tabular}{|c|c|c|c|c|}
\hline \multirow[b]{2}{*}{ Fahrzeugtyp } & \multicolumn{2}{|c|}{ Dieselbus } & \multicolumn{2}{|c|}{ Elektrobus } \\
\hline & $12 \mathrm{~m}$ & $18 \mathrm{~m}$ & $12 \mathrm{~m}$ & $18 \mathrm{~m}$ \\
\hline Tankvolumen & 2151 & $3001^{4}$ & -- & -- \\
\hline Energieinhalt ${ }^{5}$ & 2086 kWh & 2910 kWh & 250 kWh & 350 kWh \\
\hline Energieverbrauch ${ }^{6}$ & $45 \mathrm{l} / 100 \mathrm{~km}$ & $60 \mathrm{I} / 100 \mathrm{~km}^{4}$ & $2 \mathrm{kWh} / \mathrm{km}$ & $3 \mathrm{kWh} / \mathrm{km}$ \\
\hline Reichweite & $478 \mathrm{~km}$ & $500 \mathrm{~km}$ & $125 \mathrm{~km}$ & $117 \mathrm{~km}$ \\
\hline
\end{tabular}

\footnotetext{
${ }^{3}$ Soweit nicht anders angegeben, handelt es sich bei den Werten um beispielhafte Annahmen zur Darstellung des grundlegenden Zusammenhangs.

${ }^{4}$ Tschakert (2014).

${ }^{5}$ Die Berechnung des Energieinhalts des Dieselkraftstoffes basiert auf einem Heizwert von 9,7 kWh/l.

${ }^{6}$ Der Energieverbrauch von Elektrobussen ist von verschiedenen Parametern abhängig und wird in Kapitel 2.2 behandelt.
} 
Es ist zu beachten, dass es sich hierbei lediglich um eine Abschätzung handelt mit dem Ziel, eine generelle Tendenz aufzuzeigen. Sowohl für Dieselbusse als auch für Elektrobusse ist der Verbrauch von multiplen Parametern abhängig und kann für gewöhnlich nicht verallgemeinert werden. Es kann jedoch geschlussfolgert werden, dass die durchschnittliche Reichweite eines Elektrobusses nur etwa einem Viertel der Reichweite eines Dieselbusses entspricht. Die im Berechnungsbeispiel gezeigten Reichweiten sollen nun mit typischen ÖPNV-Anwendungen verglichen werden. In (Knote et al., 2017) wurden insgesamt mehr als 5000 Umlaufpläne aus 16 verschiedenen Verkehrsunternehmen analysiert und ausgewertet. Die Ergebnisse sind in Abbildung 2-1 dargestellt. Daraus geht hervor, dass vor allem bei Gelenkbussen ein Großteil der Umläufe Fahrleistungen von mehr als $200 \mathrm{~km}$ pro Tag erfordert. Bei Solobussen ist der Anteil größer als $200 \mathrm{~km}$ pro Tag etwas niedriger, jedoch beträgt er immer noch ca. 50\%. Daraus kann geschlussfolgert werden, dass für eine flächendeckende Umstellung von Dieselbussen hin zu Elektrobussen deutlich größere Reichweiten erforderlich sind, als die heutigen am Markt verfügbaren Fahrzeuge abdecken können. Zu diesem Schluss kommen auch die Autoren der Studie in (Knote et al., 2017).
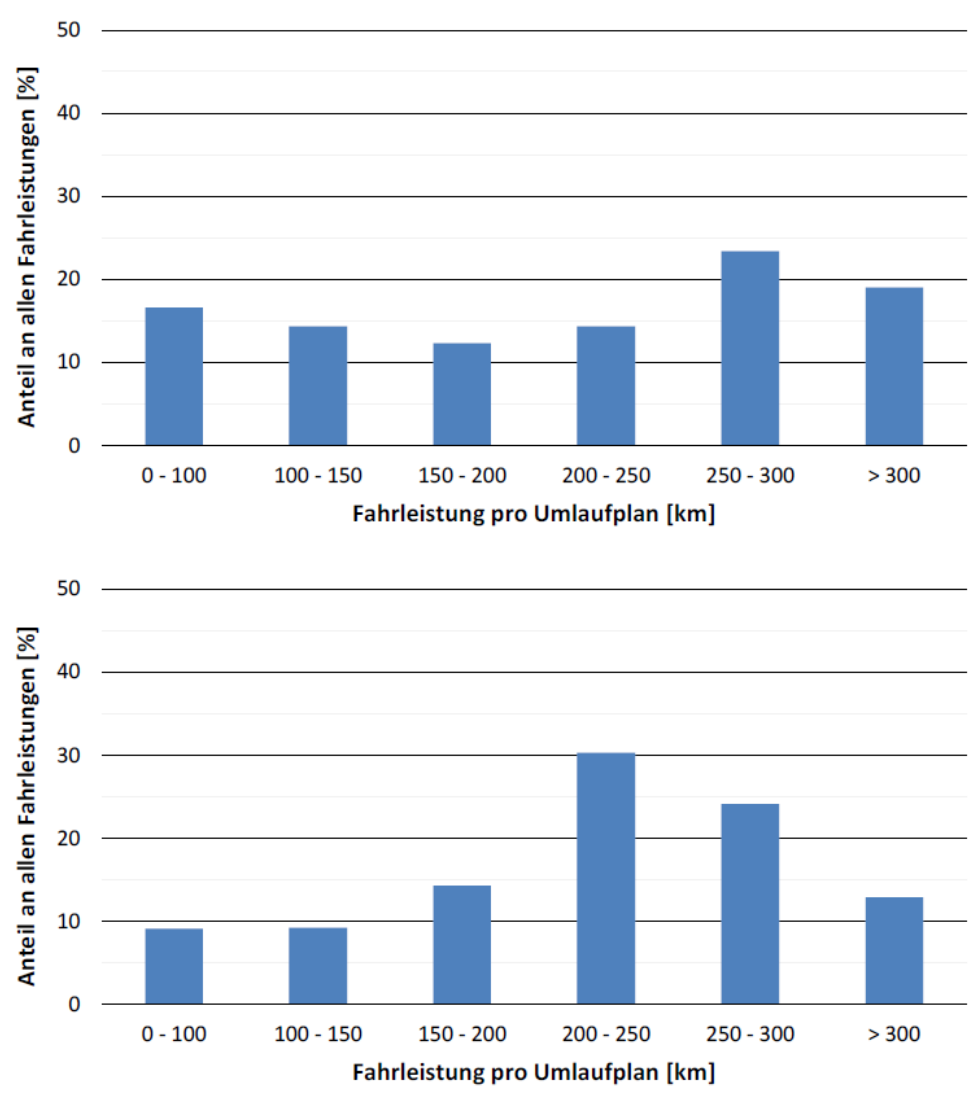

Abbildung 2-1: Typische Fahrleistungen von Bussen im ÖPNV an Werktagen, oben: Solobusse (12 m), unten: Gelenkbusse (18 m), (Knote et al., 2017) 
Daraus resultierend haben Dieselbusse hinsichtlich der täglichen Reichweite eine Verfügbarkeit von $100 \%$, d.h. sie können jede beliebige Strecke einer typischen täglichen ÖPNV-Anwendung abdecken (Vgl. Abbildung 2-1). Elektrobusse haben dagegen eine deutlich reduzierte Verfügbarkeit aus Reichweitensicht, die jedoch mittels Ladeinfrastruktur ausgeglichen werden kann. Diese wird bei Elektrobussen somit betriebsrelevant. Die Infrastruktur zum Betanken der Fahrzeuge hat bei Dieselbussen hingegen keinen nennenswerten Einfluss auf die Gewährleistung eines zuverlässigen Betriebs. Zusätzlich ist die längere Dauer des Nachladevorgangs bei Elektrobussen im Vergleich zur Betankung im Auslegungsprozess zu berücksichtigen.

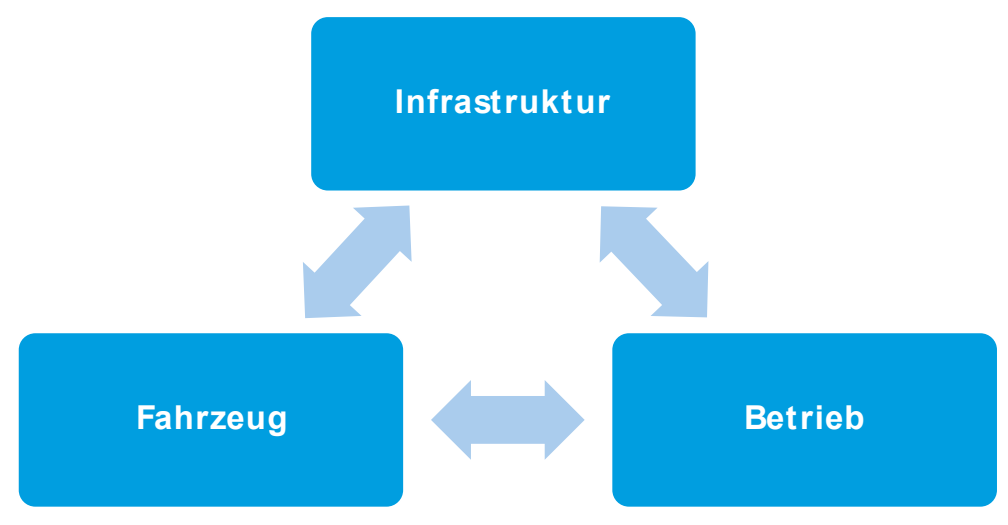

Abbildung 2-2: Hauptbestandteile im System Elektrobus, nach (Rogge et al., 2015a)

Für eine umfassende Analyse eines Elektrobuseinsatzes muss somit stets das Zusammenspiel aus Fahrzeugen, Betrieb und Ladeinfrastruktur betrachtet werden (Abbildung 2-2). Die Auslegung und Dimensionierung von Elektrobuslinien ist somit stets eine Systemauslegung. Dieser Zusammenhang ist bereits von elektrischen Bahnsystemen bekannt, in denen die Fahrzeuge die notwendige Traktionsenergie aus einer Fahrleitungsanlage beziehen.

\section{Konzepte und Technologien der Nachladung}

Als Stand der Technik haben sich zwei verschiedene Konzepte zur Nachladung von Elektrobussen etabliert.

- Übernachtladung (Depot-Charging),

- Gelegenheitsladung (Opportunity-Charging).

Die Eigenschaften beider Konzepte sind in Tabelle 2-2 vergleichend gegenübergestellt. Während die Übernachtladung nur alleinige Anwendung findet, wird die Gelegenheitsladung oftmals in Verbindung mit der Übernachtladung eingesetzt. Zusätzlich 
zum Ladekonzept haben sich mit der konduktiven und induktiven Ladung zwei verschiedene Ladetechnologien entwickelt, wobei erstere in die Ladung mit Stecker und mit Pantographen unterschieden werden kann. Tabelle 2-2 ist eine nicht vollständige Zusammenstellung wesentlicher Eigenschaften der verschiedenen Ladekonzepte und Ladetechnologien.

Tabelle 2-2: Übersicht der zwei etablierten Ladekonzepte

\begin{tabular}{ll}
\hline \multicolumn{1}{c}{ Übernachtladung } & \multicolumn{1}{c}{ Gelegenheitsladung } \\
\hline $\begin{array}{l}\text { Nachladung der Traktionsbatterie } \\
\text { ausschließlich über Nacht im Depot }\end{array}$ & $\begin{array}{l}\text { Nachladung der Traktionsbatterie } \\
\text { während des Tages an diskreten } \\
\text { Ladepunkten und zusätzlich über } \\
\text { Nacht im Depot }\end{array}$ \\
Betrieb wie mit Dieselbussen & nahezu keine Limitierung der \\
Einschränkung der Streckenwahl & Reichweite aufgrund der \\
aufgrund limitierter Reichweite & Nachlademöglichkeit während des \\
& Tages \\
& ggf. Anpassung der Betriebskonzepte \\
& aufgrund der Nachladung erforderlich \\
• konduktiv mit CCS-Stecker & Nachladung: \\
konduktiv mit Pantographen & $\bullet$ konduktiv mit Pantographen \\
\hline
\end{tabular}

Über die dargestellten Ladekonzepte und -technologien können jeweils unterschiedliche Ladeleistungen realisiert werden. Für die Ladung mit einem konventionellen CCSStecker ist die max. Ladeleistung aus thermischen Gründen auf ca. $150 \mathrm{~kW}$ begrenzt. Bei einer Erhöhung der Leistung müssen sowohl Stecker als auch das Kabel aktiv gekühlt werden. Bei konduktiver Nachladung mit einem Pantographen können bereits heute Ladeleistungen von teilweise mehr als $500 \mathrm{~kW}$ erreicht werden. Die leistungsbegrenzende Komponente ist hierbei nicht die Ladeeinrichtung (Leistungselektronik, Kontaktierung, etc.) selbst, sondern die Batterie. Die Begrenzung kann dabei sowohl thermisch als auch elektrisch bedingt sein. Mit induktiven Ladesystemen können Ladeleistungen bis ca. 200 kW realisiert werden (BMVI, 2016; Knote et al., 2017). Die Limitierung resultiert im Wesentlichen aus der kontaktlosen Energieübertragung. Für hohe Ladeleistungen sind dabei entsprechend hohe elektromagnetische Feldstärken erforderlich. Diese sind jedoch in öffentlichen Räumen gesetzlich begrenzt. Eine zusätzliche Limitierung der übertragbaren Ladeleistung resultiert aus dem eingeschränkten Bauraum für die induktiven Komponenten im Fahrzeug. 
Neben den hier präsentierten Konzepten und Technologien zur Nachladung haben sich parallel zur Entwicklung von batterie-elektrischen Bussen Oberleitungsbusse mit partieller Oberleitung entwickelt. Dafür werden u.a. die Bezeichnungen Batterie-Oberleitungsbus (BOB), In-Motion-Charging (IMC) oder Hybrid-Oberleitungsbus verwendet. Dabei handelt es sich um ein System, bei welchem die Fahrzeuge mit zweipoligen Stromabnehmern ausgerüstet sind und zusätzlich über eine (meist kleine) Traktionsbatterie verfügen. Auf Streckenabschnitten mit Oberleitung wird die Traktionsenergie aus der Fahrleitung bezogen, wobei gleichzeitig die Batterie geladen wird. In Streckenabschnitten ohne Oberleitung wird das Fahrzeug dann aus der Batterie versorgt. Derartige Systeme finden in Deutschland bisher nur wenige Anwendungen und sind nicht Betrachtungsgegenstand dieser Arbeit.

\subsection{Stand der Forschung}

Für die Einführung von Elektrobussen müssen die kommunalen Verkehrsunternehmen eine Reihe wichtiger Entscheidungen treffen. Diese sind oftmals monetär motiviert. Es ist deshalb von besonderer Wichtigkeit, dass die zugrunde liegende technische Bewertung der Systemauslegung valide ist. Diese ist ein aktuelles Forschungsthema, worauf die große Anzahl an Publikationen zu diesem Thema hinweist, welche in (Berthold, 2019) ausführlich zusammengestellt ist. Ziel der Systemauslegung ist es, die drei Hauptbestandteile des Systems Elektrobus aus Abbildung 2-2 (Fahrzeug, Ladeinfrastruktur und Betrieb) derart zu adressieren, dass deren Interaktion einen zuverlässigen Betrieb ermöglicht. Dabei haben sich im Laufe der Zeit verschiedene Herangehensweisen entwickelt.

Eine einfache Methode zur überschlägigen Systemauslegung ist die Verwendung spezifischer Verbrauchsmittelwerte. Dabei wird für ein konkretes Fahrzeug ein mittlerer Verbrauch in Form von kWh/km definiert. Über die tägliche geforderte Laufleistung kann der Tagesenergiebedarf ermittelt werden. Die Verbrauchsmittelwerte können je nach Anwendungsfall teilweise stark variieren, wobei vor allem die Fahrzeuggröße, die Topologie der Strecke oder das umgesetzte Heizungs- und Klimatisierungskonzept entscheidend sind (Ufert et al., 2018b). In Tabelle 2-3 sind einige Erfahrungswerte aus diversen Pilotprojekten angegeben.

Bei einer Ausführung als Depot-Charger (Vgl. Tabelle 2-2) resultiert der notwendige Energieinhalt der Batterie aus dem täglichen Energiebedarf der Fahrzeuge. Es besteht jedoch die Möglichkeit, dass eine solche Batteriekonfiguration hinsichtlich Gewicht oder Volumen nicht in das Fahrzeug integrierbar ist. In einem solchen Fall kann geprüft werden, ob eine Ausführung als Opportunity-Charger möglich ist. Dafür müssen sowohl ausreichend große Pausenzeiten (z.B. an den Wendestellen der Linien) als auch die Möglichkeit zur Errichtung einer oder mehrerer Ladesäulen entlang der betrachteten Strecke zur Verfügung stehen. 
Tabelle 2-3: Durchschnittliche Energieverbrauchskennzahlen (BMVI, 2016; Schwarze, 2019)

\begin{tabular}{|c|c|c|c|}
\hline Stadt & Fahrzeugtyp & $\begin{array}{l}\text { Durchschnittlicher } \\
\text { Verbrauch }\end{array}$ & Heizung \\
\hline Dresden, Linie 79 & 12 m Fahrzeug & $1,2-1,9 \mathrm{kWh} / \mathrm{km}$ & elektrisch \\
\hline Dresden, EDDA-Bus & 12 m Fahrzeug & $1,19-1,25 \mathrm{kWh} / \mathrm{km}$ & $\begin{array}{c}\text { fossile } \\
\text { Zusatzheizung }\end{array}$ \\
\hline Köln & 18 m Fahrzeug & $1,4-3,45 \mathrm{kWh} / \mathrm{km}$ & elektrisch \\
\hline Braunschweig & 18 m Fahrzeug & $2,3-3,1 \mathrm{kWh} / \mathrm{km}$ & $\begin{array}{c}\text { fossile } \\
\text { Zusatzheizung }\end{array}$ \\
\hline
\end{tabular}

Eine Beispielrechnung für zwei verschiedene Auslegungsvarianten ist in Tabelle 2-4 gegeben. Darin wird jeweils eine Linie betrachtet, welche in sechs Umläufen eine Tagesfahrleistung von $228 \mathrm{~km}$ aufweist. Der spezifische Energiebedarf sei mit 2,1 kWh/km angenommen. Bei einer Auslegung als Depot-Charger muss definitionsgemäß die komplette Energie in der Batterie speicherbar sein. Damit ergibt sich ein Energiebedarf von $479 \mathrm{kWh}$. Aus Gründen der Batteriebelastung wird jedoch oftmals empfohlen, die verfügbare Batteriekapazität nur zu ca. $80 \%$ zu nutzen. Damit ergibt sich bereits ein zu installierender Batterieenergieinhalt von $599 \mathrm{kWh}$. Unter Berücksichtigung der Tatsache, dass eine Batterie im Laufe ihres Lebens bis zu 20\% an Kapazität verlieren kann, muss diese mit einem Energieinhalt von $748 \mathrm{kWh}$ dimensioniert werden, wenn das Fahrzeug auch am Ende der Batterielebensdauer die geplante Tagesfahrleistung bewältigen können soll.

Bei einer Auslegung als Opportunity-Charger müssen sowohl die Ladehäufigkeiten und die Ladezeiten als auch die Ladeleistung definiert werden. Im Beispiel in Tabelle 2-4 wird die Realisierung eines Ladepunktes untersucht, beispielsweise an einem der Wendepunkte der Linie. Dieser wird am Tag sechsmal angefahren und es stehen jeweils 15 min Ladezeit zur Verfügung. Dabei soll mit $300 \mathrm{~kW}$ nachgeladen werden. Der im Beispiel des Depot-Chargers ermittelte Energieinhalt von 748 kWh kann somit um die Tagesenergiemenge der Nachladung reduziert werden. Damit ergibt sich ein zu installierender Energieinhalt der Batterie von $298 \mathrm{kWh}$. Dies stellt eine große, aber durchaus realisierbare Traktionsbatterie für einen $18 \mathrm{~m}$ langen Gelenkbus dar, wie aktuell am Markt verfügbare Fahrzeuge zeigen (SILEO, 2019).

Aus der Beispielrechnung in Tabelle 2-4 geht hervor, dass grundsätzlich alle Bestandteile des Systems Elektrobus (Vgl. Abbildung 2-2) adressiert wurden. Es wird jedoch auch ersichtlich, dass es sich bei dieser Methode um eine Energiebilanzierung handelt. Das heißt, dass zwar eine Aussage zur grundsätzlichen Bewältigung der täglichen Fahrleistung getroffen werden kann. Jedoch basiert diese Aussage auf der Annahme, 
dass die Batteriebelastung möglichst gleichmäßig auftritt. Aussagen zur elektro-thermischen Belastung und zur Batteriealterung bei dynamischen Fahrszenarien sind damit nicht möglich. Für die Betrachtung solcher Effekte wird nicht nur der Energiebedarf des Fahrzeugs, sondern der Leistungsbedarf benötigt. Dies erfordert ein valides Fahrzeugmodell, welches den Leistungsbedarf für ein konkretes Anwendungsszenario simulativ ermittelt. Dabei sind neben der Topologie der Strecke auch konkrete Wetterdaten erforderlich.

Tabelle 2-4: Beispielrechnung für zwei verschiedene Auslegungsvarianten mittels spezifischer Verbrauchsmittelwerte

\begin{tabular}{|c|c|c|}
\hline & $\begin{array}{l}\text { Auslegung als } \\
\text { Depot-Charger }\end{array}$ & $\begin{array}{c}\text { Auslegung als } \\
\text { Opportunity-Charger }\end{array}$ \\
\hline Tagesfahrleistung & \multicolumn{2}{|c|}{228 km (aufgeteilt auf 6 Umläufe) } \\
\hline $\begin{array}{l}\text { Energieverbrauch des } \\
\text { Fahrzeugs (Gelenkbus, } 18 \mathrm{~m} \text { ) }\end{array}$ & \multicolumn{2}{|c|}{$2,1 \mathrm{kWh} / \mathrm{km}$} \\
\hline Nachladung auf der Strecke & $\begin{array}{l}\text { keine, } \\
\text { nur Depot- } \\
\text { Charging }\end{array}$ & $\begin{array}{l}1 \text { Ladepunkt am Wendepunkt } \\
\text { Ladezeit: } 15 \text { min } \\
\text { Ladehäufigkeit: } 6 \text { mal pro Tag } \\
\text { Ladeleistung: } 300 \mathrm{~kW}\end{array}$ \\
\hline $\begin{array}{l}\text { Gesamtenergiebedarf eines } \\
\text { Fahrzeugs pro Tag }\end{array}$ & \multicolumn{2}{|c|}{$2,1 \frac{\mathrm{kWh}}{\mathrm{km}} \cdot 228 \mathrm{~km}=479 \mathrm{kWh}$} \\
\hline $\begin{array}{l}\text { Limitierung der nutzbaren } \\
\text { Batteriekapazität auf } 80 \% \\
\text { der verfügbaren Kapazität }\end{array}$ & \multicolumn{2}{|c|}{$\frac{479 k W h}{0,8}=599 k W h$} \\
\hline $\begin{array}{l}\text { Berücksichtigung eines } \\
\text { Kapazitätsverlusts von } 20 \% \\
\text { während der Nutzungszeit } \\
\text { der Batterie }\end{array}$ & \multicolumn{2}{|c|}{$\frac{599 k W h}{0,8}=748 k W h$} \\
\hline $\begin{array}{l}\text { Nachgeladene Energie am } \\
\text { Ladepunkt (pro Tag) }\end{array}$ & -- & $6 \cdot 15 \mathrm{~min} \cdot 300 \mathrm{~kW}=450 \mathrm{kWh}$ \\
\hline $\begin{array}{l}\text { Zu installierender } \\
\text { Energieinhalt der Batterie }\end{array}$ & $748 \mathrm{kWh}$ & $748 k W h-450 k W h=\mathbf{2 9 8} \boldsymbol{k W h}$ \\
\hline
\end{tabular}

Ein mögliches Vorgehen zur Leistungsbedarfsermittlung eines Elektrobusses auf einer vorgegebenen Strecke ist in (Bunzel et al., 2018) detailliert beschrieben und demonstriert. Als Ergebnis der Fahrzeugsimulation ergibt sich der elektrische Leistungsbedarf am Zwischenkreis, welcher von der Traktionsbatterie realisiert werden muss. Dieser 
kann nun als Eingangsgröße für eine Batteriesimulation verwendet werden. Die Traktionsbatterie stellt allgemein bei Elektrofahrzeugen zurzeit die teuerste und sensibelste Einzelkomponente dar (Kampker et al., 2018), weshalb eine detaillierte Betrachtung zur Beurteilung der Wirtschaftlichkeit und Effizienz des Gesamtsystems zwingend erforderlich ist. Dazu zählen neben der elektro-thermischen Belastung auch Untersuchungen zum Alterungsverhalten der Traktionsbatterie unter realen Belastungsszenarien.

In Tabelle 2-5 sind ausgewählte Forschungsaktivitäten und deren Kernpunkte zur Systemauslegung und Bewertung von Elektrobuslinien bzw. Elektrobusnetzen gegeben. Die Quellen sind hauptsächlich hinsichtlich der oben beschriebenen Ansätze zur Batteriedimensionierung analysiert.

In (Kunith, 2017) und (Jefferies et al., 2018) wurde eine Methodik entwickelt, mit der verschiedene Elektrifizierungstechnologien für Bussysteme hinsichtlich Kosteneffizienz und Emissionen verglichen und bewertet werden können. Dabei stehen batterieelektrische Bussysteme im Vordergrund, es sind jedoch auch Oberleitungsbusse oder wasserstoffbetriebene Fahrzeuge Bestandteil der Betrachtungen. In (Kunith, 2017) wird ein umfangreiches Modell zur technisch-wirtschaftlichen Bewertung entwickelt. Während der wirtschaftliche Anteil mittels eines TCO-Modells (Total Cost of Ownership) sehr ausführlich dargestellt wird, basiert die technische Bewertung auf der Methode der Energiebilanzierung. Eine Fahrzeug- und Batteriesimulation ist nicht $\mathrm{Be}-$ standteil der Arbeit. So wird beispielsweise die Batterielebensdauer in beiden genannten Quellen für alle betrachteten Szenarien mit 6 Jahren angenommen. Wie im weiteren Verlauf dieser Arbeit noch gezeigt werden wird, führt diese Annahme vor allem beim Vergleich von Depot-Ladern und Gelegenheitsladern zu einer Verzerrung der Ergebnisse.

In (Berthold, 2019) wurde ebenfalls ein Ansatz zur Berechnung der TCO gewählt. Darin werden ausschließlich batterie-elektrische Fahrzeuge untersucht und es findet vorwiegend eine Betrachtung einzelner Linien statt. Am Ende der Arbeit wird ein Ansatz zu netzweiten Untersuchungen skizziert, jedoch nicht vollständig entwickelt. Die energetische Betrachtung basiert auf einer Fahrzeugsimulation und dem daraus resultierenden Leistungsbedarf. Der zugrundeliegende Geschwindigkeitsverlauf wird in einer aufwändigen Verkehrsflusssimulation ermittelt. Die Batteriesimulation basiert hingegen auf einem sehr einfachen Ansatz. Es findet nur eine elektrische, jedoch keine thermische Batteriesimulation statt. Des Weiteren ist kein valides Alterungsmodell integriert. Die Bestimmung der resultierenden Batteriealterung basiert lediglich auf Angaben aus Zelldatenblättern. Dabei wird ausschließlich die Belastung durch den SOC-Hub betrachtet und mittels einer empirischen Korrekturformel an die ermittelte elektrische Belastung angepasst. Wie im weiteren Verlauf dieser Arbeit noch gezeigt werden wird, sind neben dem SOC-Hub eine Reihe weiterer Faktoren zur Bestimmung der Batteriealterung in Elektrofahrzeugen notwendig. Dazu zählt unter anderem die 
Batterietemperatur, was wiederum die Ermittlung der thermischen Belastung erfordert. Die Arbeit beinhaltet eine integrierte Optimierungsfunktion, welche die Parameter Batteriegröße, Standort der Ladepunkte entlang der Strecke und Ladeleistung variiert. Dabei werden stets weniger als 5 Variationen je Parameter zugelassen, sodass insgesamt nur wenige Hundert verschiedene Auslegungsvarianten berechnet, bewertet und verglichen werden können.

Tabelle 2-5: Übersicht Forschungsaktivitäten

\begin{tabular}{|c|c|c|}
\hline Institution & Referenzquelle(n) & Kernpunkte \\
\hline TU Berlin & $\begin{array}{l}\text { (Kunith, 2017) } \\
\text { (Jefferies et al., } \\
\text { 2018) }\end{array}$ & $\begin{array}{l}\text { - Basis: Energiebilanzierung } \\
\text { - keine Fahrzeug- oder Systemsimulation } \\
\text { - keine Alterungsberechnung } \\
\text { - ausführliche TCO-Berechnung }\end{array}$ \\
\hline KIT Karlsruhe & (Berthold, 2019) & $\begin{array}{l}\text { - Basis: detaillierte Fahrzeugsimulation zur } \\
\text { - einergie- und Leistungsbedarfsermittlung } \\
\text { - keine thermische Batteriesimulation } \\
\text { - sehr einfache Alterungsabschätzung } \\
\text { - ausführliche TCO-Berechnung }\end{array}$ \\
\hline $\begin{array}{l}\text { FhG IVI, } \\
\text { Dresden }\end{array}$ & $\begin{array}{l}\text { (Steinert, 2015) } \\
\text { (Fichtl et al., 2016) }\end{array}$ & $\begin{array}{l}\text { - Basis: Fahrzeugsimulation mit elektrischer } \\
\text { Batteriesimulation (Detaillierungsgrad } \\
\text { unbekannt) } \\
\text { - } \text { keine thermische Batteriesimulation } \\
\text { - keine Alterungsberechnung } \\
\text { - Grobanalyse von Busnetzen und } \\
\text { Detailanalyse von Buslinien }\end{array}$ \\
\hline $\begin{array}{l}\text { RWTH } \\
\text { Aachen }\end{array}$ & $\begin{array}{l}\text { (Rogge et al., } \\
2015 \mathrm{a}) \\
\text { (Rogge et al., } \\
2015 \mathrm{~b})\end{array}$ & $\begin{array}{l}\text { - Basis: Energiebilanzierung } \\
\text { - keine Fahrzeugsimulation } \\
\text { - keine Batteriesimulation } \\
\text { - keine Alterungsberechnung } \\
\text { - Analyse kompletter Busnetze }\end{array}$ \\
\hline
\end{tabular}


In (Steinert, 2015) und (Fichtl et al., 2016) wurde eine Simulationsumgebung zur Elektrifizierung von Stadtbussen mittels eines mehrstufigen Verfahrens zur Ermittlung einer konkreten Systemauslegung entwickelt. In einer ersten Grobanalyse werden zunächst alle Strecken eines lokalen Busnetzes hinsichtlich der generellen technischen Machbarkeit einer Umstellung von Dieselbussen auf Elektrobusse analysiert. Die Quelle liefert hier jedoch keine Informationen zur verwendeten Methodik bzw. zum Grad der Automatisierung. Anschließend erfolgt Priorisierung der Strecken, die nun einer detaillierten Analyse unterzogen werden. Darin können neben batterie-elektrischen Fahrzeugen auch weitere Fahrzeugkonzepte (z.B. Hybridbusse oder Batteriebusse mit Range Extender) untersucht werden. Die Quelle gibt keinerlei Informationen über die Methodik oder die Granularität der Fahrzeugsimulation sowie der Batteriesimulation. Die Aussagen der Quelle lassen jedoch vermuten, dass lediglich die elektrische Belastung der Batterie ermittelt wird. Die resultierende thermische Belastung als auch das Alterungsverhalten werden in der Quelle nicht erwähnt.

In (Rogge et al., 2015a) und (Rogge et al., 2015b) wurde eine Methodik entwickelt, mit welcher komplette Busnetze hinsichtlich einer teilweisen bzw. vollständigen Flottenumstellung analysiert werden können. Dafür werden alle Umläufe des zu betrachtenden Busnetzes hinsichtlich der technischen Machbarkeit untersucht. Auf Basis der daraus gewonnenen Erkenntnisse wird eine Roadmap zur angestrebten Flottenumstellung aufgestellt. Die Ermittlung des Energiebedarfs basiert hierbei auf der Methode der Energiebilanzierung. Eine Fahrzeugsimulation und eine Batteriesimulation werden nicht durchgeführt.

\subsection{Ableitung des Forschungsbedarfs}

Aus den aufgeführten Quellen in Kapitel 2.2 kann geschlussfolgert werden, dass im Prozess der Systemauslegung von Elektrobussen eine Vielzahl von Zwischenschritten erforderlich ist. Diese können chronologisch wie folgt zusammengefasst werden:

1) Ermittlung des Energie- und Leistungsbedarfs der Fahrzeuge zur Bewältigung einer oder mehrerer Strecken bzw. Linien, gegebenenfalls mittels Fahrzeugsimulation,

2) ganzheitliche Batteriesimulation inkl. verschiedener Nachladeszenarien und technologien,

3) Berechnung der Total Cost of Ownership,

4) Systemoptimierung.

Aus den Ausführungen in Kapitel 2.2 geht hervor, dass von den aufgezählten Punkten vor allem die Punkte zwei und vier bisher nur unzureichend abgedeckt werden. Es wird 
lediglich in einer Quelle (Berthold, 2019) eine elektrische Simulation der Batterie durchgeführt. Die Modellierung basiert dabei jedoch auf einer Spannungsquelle und einem konstanten ohmschen Innenwiderstand. Wie im weiteren Verlauf der Arbeit noch gezeigt werden wird, sind für eine valide Batteriesimulation sowohl eine tiefere Modellierung als auch eine detailliertere Parametrierung der Batterie erforderlich. Beispielsweise ist die Kapazität der Batterie temperaturabhängig, wodurch die Notwendigkeit einer thermischen Batteriesimulation bereits gegeben ist. Diese wird von keiner der oben aufgeführten Quellen berücksichtigt. Des Weiteren wird im Verlauf der Arbeit gezeigt, dass zur Beurteilung der Wirtschaftlichkeit die Kenntnis über die Batterielebensdauer von elementarer Bedeutung ist. Der Alterungsprozess der Batterie wird von zahlreichen Faktoren beeinflusst, weshalb eine detaillierte Simulation erforderlich ist. Eine ganzheitliche Batteriesimulation umfasst demnach mehrere Teilaspekte, welche stets im Zusammenspiel zu betrachten sind.

Des Weiteren wird der Punkt der Optimierung von den oben aufgeführten Quellen nur unzureichend abgedeckt. Lediglich in (Berthold, 2019) können wenige Hundert verschiedene Auslegungsszenarien automatisiert berechnet und miteinander verglichen werden. Für eine optimale Auslegung einer Elektrobuslinie ist es von Vorteil, wenn möglichst viele Varianten berechnet und miteinander verglichen werden können. Aus Effizienzgründen ist dafür ein automatisierter Prozess erstrebenswert.

Der Fokus dieser Arbeit wird aus den genannten Gründen auf eine detaillierte ganzheitliche Batteriesimulation inkl. verschiedener Nachladeszenarien und einer automatisiert ablaufenden Optimierungsfunktion gelegt. Die übrigen beiden oben genannten Punkte im Prozess der Systemauslegung (Leistungsbedarfsermittlung und TCO-Berechnung) werden, wie dargelegt, bereits in anderen Arbeiten ausführlich behandelt. Mit den Ergebnissen dieser Arbeit soll dennoch ein vollständiger Systemauslegungsprozess einer Elektrobuslinie möglich sein, weshalb die Punkte Ermittlung des Fahrzeugleistungsbedarfs und Kostenberechnung ebenfalls berücksichtigt werden, jedoch mit niedrigerer Granularität. Für die ökonomische Betrachtung wird in Kapitel 4.3 ein einfaches Kostenmodell entwickelt. Im weiteren Verlauf der Arbeit wird noch erläutert, dass dieses Modell nicht zur TCO-Berechnung, sondern lediglich zur Unterscheidbarkeit einzelner Szenarien gedacht ist. Für die Ermittlung des Leistungsbedarfs wird auf ein bereits vorhandenes Fahrzeugmodell zurückgegriffen, welches zwischen 2016 und 2019 im Rahmen des Forschungsprojektes EKE-ÖPNV an der Professur für Fahrzeugmechatronik der Technischen Universität Dresden entstand und in (Bunzel et al., 2018) ausführlich beschrieben ist. Darin wurde eine Methodik entwickelt, welche mittels eines hybriden Fahrzeugmodells die Hauptbestandteile des Leistungsbedarfs eines Elektrobusses (Antrieb, Heizung und Klima, sonstige Nebenverbraucher sowie Beladung durch Fahrgäste) auf einer gegebenen Strecke ermittelt 
und als zeitlichen Verlauf auf Basis von Mikrozeitschritten ${ }^{7}$ ausgibt. Es handelt sich dabei um einen rückwärtsgerichteten Modellansatz. Bei einem Rückwärtsmodell wird der Leistungsbedarf des Fahrzeugs ausgehend von einem vorgegebenen Geschwindigkeitsprofil berechnet. Die Traktionskomponenten sind derart zu konzeptionieren und zu dimensionieren, dass die Anforderungen zu jedem Zeitpunkt erbracht werden können. Der Einfluss eines Fahrers wird bei Rückwärtsmodellen in der Regel nicht berücksichtigt.

In der vorliegenden Arbeit wird eine Umgebung zur Systemsimulation von Elektrobuslinien entwickelt mit dem Ziel, eine möglichst kosteneffiziente Auslegungsvariante zu ermitteln. Dafür wird in die Simulationsumgebung eine Optimierungsfunktion integriert. Die konkreten Ziele der Arbeit sind in Tabelle 2-6 zusammengefasst.

\section{Tabelle 2-6: Zusammenfassung der Forschungsziele}

Entwicklung einer Simulationsumgebung zur Systemauslegung
einzelner Elektrobuslinien
detaillierte Modellierung und Simulation der Traktionsbatterie
mittels eines elektrischen, eines thermischen und eines
Alterungsmodells
automatisierte Berechnung einer Vielzahl an
Auslegungsvarianten
integrierte Optimierungsfunktion zur Ermittlung der optimalen
Auslegungsvariante

Mit Hilfe der zu entwickelnden Simulationsumgebung sollen anschließend folgende Forschungsfragen beantwortet werden können:

- Welche elektro-thermische Belastung erfährt die Traktionsbatterie im Linieneinsatz eines Elektrobusses und welche Lebensdauererwartung resultiert daraus?

- Welcher Energieinhalt der Traktionsbatterie in Kombination mit welcher Nachladestrategie führt zum wirtschaftlichen Optimum?

- Welche Kostenbestandteile unterscheiden verschiedene Systemkonfigurationen und wie wirken sich Änderungen der Kostensätze auf die Systemkosten aus?

- Welche Auswirkungen hat der zu erwartende Betrieb auf die optimale Auslegungsvariante?

\footnotetext{
${ }^{7}$ Für energetische Fahrzeugsimulationen werden oftmals Zeitschrittweiten zwischen $0,1 \mathrm{~s}$ und 1,0 s verwendet.
} 


\section{$3 \quad$ Konzept zur simulationsbasierten Systemauslegung einer Elektrobuslinie}

In diesem Kapitel wird ein Konzept zur Umsetzung der in Kapitel 2.3 definierten Forschungsziele entwickelt. Dafür werden zunächst die im Rahmen dieser Arbeit relevanten Freiheitsgrade in der Systemauslegung einer Elektrobuslinie hergeleitet, auf denen die anschließende Konzeptentwicklung der Simulationsumgebung mit integrierter Optimierungsfunktion basiert.

\subsection{Herleitung wesentlicher Freiheitsgrade in der Systemauslegung}

Für die Systemauslegung einer Elektrobuslinie ergibt sich eine Vielzahl an möglichen Freiheitsgraden, welche innerhalb technischer Grenzen beliebig miteinander kombinierbar sind. In diesem Abschnitt werden die für diese Arbeit relevanten Freiheitsgrade des Systems definiert, welche anschließend in der Simulationsumgebung umgesetzt werden. Grundlegend können diese sowohl im Bereich der technischen Hauptbestandteile (Fahrzeug und Ladeinfrastruktur) als auch im betrieblichen Bestandteil des Systems (Fahrplan, Umlaufplan) liegen (Vgl. Abbildung 2-2).

\section{Fahrzeug}

Fahrzeugseitig liegt der Fokus in dieser Arbeit auf der Simulation und damit auf der Modellierung und Parametrierung der Batterie. Die Wahl des Batterietyps sowie der Aufbau und die Konfiguration bestimmen maßgeblich deren Leistungsfähigkeit. Neben der Batterie bietet der Bestandteil Fahrzeug weitere Teilbereiche, welche im Rahmen einer Systemauslegung betrachtet werden können. In (Liebers et al., 2016) wurde am Beispiel eines Hybridbusses mit seriellem Antriebsstrang gezeigt, dass mittels eines effizienten Heizungs- und Klimatisierungskonzepts der Energieverbrauch des Fahrzeugs reduziert werden kann. Des Weiteren geht aus (Rudat, 2019) und (Boettcher, 2019) hervor, dass sowohl der Fahrstil des Fahrers als auch der Straßenverkehr einen erheblichen Einfluss auf den Energiebedarf haben. Die genannten Teilbereiche werden jedoch in anderen Forschungsarbeiten ausführlich behandelt und sind nicht Bestandteil dieser Arbeit.

Als wesentlicher Freiheitsgrad des Fahrzeugs wird die Dimensionierung der Traktionsbatterie definiert. Die entscheidende Größe ist dabei der verfügbare Energieinhalt, da dieser maßgeblich über die Reichweite und damit über einen sensiblen Auslegungspunkt, vor allem im Vergleich zu Dieselbussen, entscheidet. Je größer diese gewählt 
wird, desto größer ist die Reichweite des Fahrzeugs. Jedoch steigt dabei auch der Energieverbrauch aufgrund des höheren Gewichts bei gleichzeitiger Reduzierung der Anzahl zulässiger Fahrgäste, da das maximal zulässige Fahrzeuggesamtgewicht gesetzlich geregelt ist. Laut $\$ 34$ Absatz 5 der Straßenverkehrs-Zulassungs-Ordnung gilt für Solobusse (12 m) ein Grenzwert von 19,5 t und für Gelenkbusse (18 m) ein Wert von $28 \mathrm{t}$. Wird demnach eine kleinere Batterie verwendet, steigt die Anzahl zulässiger Fahrgäste, jedoch wird nun die Reichweite des Fahrzeugs eingeschränkt. In Kapitel 2.1 wurde gezeigt, das vor allem die geringe Reichweite aktuell einen entscheidenden Nachteil von Elektrobussen gegenüber Dieselbussen darstellt. Dieser Nachteil kann zumindest teilweise mittels zweierlei Maßnahmen ausgeglichen werden. Einerseits kann die Anzahl an Fahrzeugen erhöht werden, welche zur Sicherstellung der geforderten Tagesfahrleistung erforderlich ist. Dies stellt jedoch eine triviale Lösung dar und ist deshalb nicht Bestandteil der Betrachtungen in dieser Arbeit. Zum anderen können die Fahrzeuge während des Tages nachgeladen und somit die tägliche Reichweite entscheidend erhöht werden.

\section{Ladeinfrastruktur und Betrieb}

Die Nachladung stellt demnach den 2. Freiheitsgrad neben der Batteriedimensionierung dar. Die nachzuladende Energiemenge kann über die folgenden Parameter gesteuert werden:

- Ladezeit einer einzelnen Nachladung,

- Häufigkeit der Nachladung je Betriebstag,

- Ladeleistung je Nachladevorgang.

In dieser Arbeit wird nur die Lademöglichkeit im Stillstand betrachtet, eine Ladung während der Fahrt über eine Oberleitung ist nicht Gegenstand der Betrachtungen. Als mögliche Ladestandorte kommen somit nur Haltestellen, Wendepunkte sowie das Busdepot in Frage.

An dieser Stelle müssen die Betriebsabläufe eines Verkehrsunternehmens zwingend beachtet werden. Dies ist vor allem bei einer möglichen Nachladung an Wendepunkten interessant. Die sog. Wendezeit wird oftmals als Pausenzeit für Fahrer genutzt und ist dementsprechend länger als Haltezeiten an regulären Haltestellen. Ist am Linienende keine Pause für den Fahrer notwendig, wird diese aus Kostengründen meist möglichst kurzgehalten. Die Wendezeit, welche auch als potentielle Ladezeit dient, ist somit aus den übergeordneten Betriebsabläufen vorgegeben und sollte aus Sicht von Verkehrsunternehmen nach Möglichkeit nicht verändert werden. Diese Prämisse wird in dieser Arbeit übernommen. Neben den Wendestellen kann eine Nachladung an ausgewählten Haltestellen erfolgen. Dabei wird innerhalb kürzester Zeit eine Pulsladung 
mit teilweise sehr hohen Leistungen realisiert, beispielsweise beim TOSA-System in Genf (Holz et al., 2017). Es ist jedoch zu beachten, dass die Haltezeit an regulären Haltestellen oftmals innerhalb weniger Zehnsekunden liegt. Beim Beispiel des TOSASystems in Genf beträgt die Nachladezeit an einer Haltestelle durchschnittlich $15 \mathrm{~s}$. Aufgrund der dargelegten Gründe wird für diese Arbeit definiert, dass der Betrieb (und damit die Ladezeit) keinen Freiheitsgrad, sondern lediglich eine Randbedingung für die Systemsimulation und die Optimierung darstellt.

Unabhängig davon, ob die Nachladung an einem Wendepunkt oder einer regulären Haltstelle realisiert wird, gilt es zu beachten, dass die jeweilige Haltezeit $t_{\text {Halt }}$ bzw. Wendezeit $t_{\text {Wende }}$ nicht automatisch die verfügbare Ladezeit darstellen. Vielmehr müssen die Rüstzeit $t_{\text {rüst }}$ (Anschluss des Fahrzeugs an den Ladepunkt, sog. Kontaktierung) sowie eventuelle Verspätungen $t_{\text {Verspätung }}$ berücksichtigt werden. Die verfügbare Ladezeit $t_{\text {Laden }}$ an einem Wendepunkt ergibt sich nach Formel (3-1). Für die verfügbare Ladezeit an einer Haltestelle ist statt der Wendezeit die Haltezeit $t_{\text {Halt }}$ einzusetzen.

$$
t_{\text {Laden }}=t_{\text {Wende }}-2 \cdot t_{\text {rüst }}-t_{\text {Verspätung }}
$$

Für die Berücksichtigung von Verspätungen bestehen im Wesentlichen zwei Möglichkeiten. Zum einen kann eine mittlere Verspätung angenommen werden. Dabei wird die durchschnittliche Verspätung eines Tages auf die Wendezeiten umgelegt und die verfügbare Ladezeit bei jedem Ladevorgang entsprechend verkürzt. Die dafür notwendigen Statistiken liegen in der Regel bei den Verkehrsunternehmen vor. Eine weitere Möglichkeit ist die Anwendung eines $(n-1)$ Betriebsszenarios. Dabei ist $n$ die Anzahl der täglichen Ladevorgänge an den Wendepunkten, von denen einer aufgrund einer Verspätung nicht realisiert werden kann. Praktisch bedeutet dies, dass die Verspätung am Ladepunkt derart groß ist, dass nach Abzug der Rüstzeiten keine Ladezeit mehr zur Verfügung stehen würde. Je nach gewünschter Systemverfügbarkeit können mehrere solcher Szenarien pro Tag berücksichtigt werden.

Für den Freiheitsgrad der Nachladung verbleiben von den oben aufgezählten Optionen somit nur noch zwei Möglichkeiten: die Häufigkeit der Nachladung und die Ladeleistung. Die Häufigkeit der Ladung kann über die Anzahl (und die Position) der Ladepunkte im Netz direkt gesteuert werden. Die nachgeladene Energiemenge pro Ladevorgang kann (bei betrieblich feststehender Ladezeit) über die Ladeleistung gesteuert werden.

\section{Zusammenfassung}

Zusammenfassend werden folgende Freiheitsgrade für die Systemauslegung einer Elektrobuslinie definiert, welche im Rahmen dieser Arbeit betrachtet werden. 
- Energieinhalt der Batterie (bzw. Kapazität der Batterie),

- Anzahl und Positionen der Nachladepunkte entlang der Strecke,

- zu installierende Leistung an den Nachladepunkten.

Diese Freiheitsgrade werden im weiteren Verlauf der Arbeit genutzt, um verschiedene Auslegungsvarianten einer Elektrobuslinie zu definieren, zu simulieren und zu bewerten. Eine konkrete Ausführung, also die Zusammensetzung aus einer bestimmten Batteriegröße, definierten Orten der Nachladung sowie einer konkreten Ladeleistung, wird in dieser Arbeit als Systemkonfiguration bezeichnet.

Neben der Nachladung an Ladepunkten auf der Strecke besteht außerdem die Möglichkeit der Ladung im Depot (Vgl. Tabelle 2-2). In dieser Arbeit wird vorausgesetzt, dass die Fahrzeuge stets im Depot geladen werden. Somit wird die Depotladung nicht als Freiheitsgrad betrachtet. Es wird davon ausgegangen, dass die Fahrzeuge unabhängig vom Ladekonzept entlang der Strecke stets im Depot auf den maximalen SOC geladen werden. Dieser kann in der Systemsimulation frei definiert werden.

\subsection{Entwicklung des Simulationskerns der Systemsimulation}

Der in diesem Abschnitt zu entwickelnde Simulationskern soll sowohl für die Systemsimulation als auch für die zu integrierende Optimierungsfunktion anwendbar sein. Dafür sind nacheinander zwei grundlegende Fragestellungen zu beantworten:

1. Mit welchen Systemkonfigurationen kann eine konkrete Linie mit Elektrobussen betrieben werden?

2. Welche dieser Systemkonfigurationen stellt die wirtschaftlich optimale Konfiguration dar?

Die erste Fragestellung adressiert die technische Machbarkeit. Dabei wird zunächst geprüft, ob mit aktuellen Technologien eine technische Realisierbarkeit möglich ist. Hierbei ist vor allem die Ermittlung der verfügbaren Reichweite unter gegebenen betrieblichen Bedingungen von hoher Bedeutung. Nur mittels einer validen Reichweitenprognose kann die technische Machbarkeit zuverlässig bestimmt werden. Die zweite Fragestellung adressiert das effiziente Zusammenspiel der einzelnen Komponenten einer Systemkonfiguration. Für dessen Ermittlung wird gemäß den definierten Forschungszielen eine automatisiert ablaufende Optimierungsfunktion in die Systemsimulation integriert.

Den Kern der Systemsimulation stellt die Batteriesimulation dar. Es wird somit ein detailliertes und valides Batteriemodell benötigt. Es wurde bereits skizziert, dass dies 
sowohl eine elektro-thermische Simulation als auch eine Alterungsberechnung beinhalten muss. Aus der Literatur sind derartige Ansätze bereits bekannt. Beispielsweise wurde in (Fleckenstein, 2013) ein dreistufiges Batteriemodell zur Simulation von ElektroPKW entwickelt. In (Schmalstieg et al., 2014) wird ein Alterungsmodell entwickelt, welches anschließend in eine vollständige Batteriesimulation eingebettet wird. Jedoch ist in beiden genannten Quellen das zeitliche Zusammenspiel der einzelnen Teilmodelle nur unzureichend bzw. nicht erläutert und demonstriert.

Für diese Arbeit wird ebenfalls der Ansatz eines dreistufigen Batteriemodells gewählt. Neben der Modellierung der einzelnen Teilmodelle wird dabei auch das zeitliche Zusammenspiel spezifiziert. Der Aufbau des Simulationskerns ist in Abbildung 3-1 gegeben. Darin sind die drei Einzelmodelle elektrisches Modell, thermisches Modell und Alterungsmodell sichtbar. Aus der Abbildung geht außerdem hervor, dass die Teilmodelle aufeinander wirken und rückwirken. Beispielsweise hat die elektro-thermische Belastung einen Einfluss auf das Alterungsmodell, während das thermische Modell und das Alterungsmodell auf das elektrische Modell zurückwirken. Zusätzlich können die Teilmodelle mit Randbedingungen zum Steuern einzelner Effekte versehen werden, beispielsweise:

- Batteriemanagementfunktionen (BMS-Funktionen) für das elektrische Modell, (In dieser Arbeit wird eine mehrdimensionale Strombegrenzung $I_{\text {Batt,lim }}$ implementiert.)

- verschiedene Kühlungs- oder Heizstrategien im thermischen Modell. (In dieser Arbeit werden Kühlung und Heizung implementiert.)

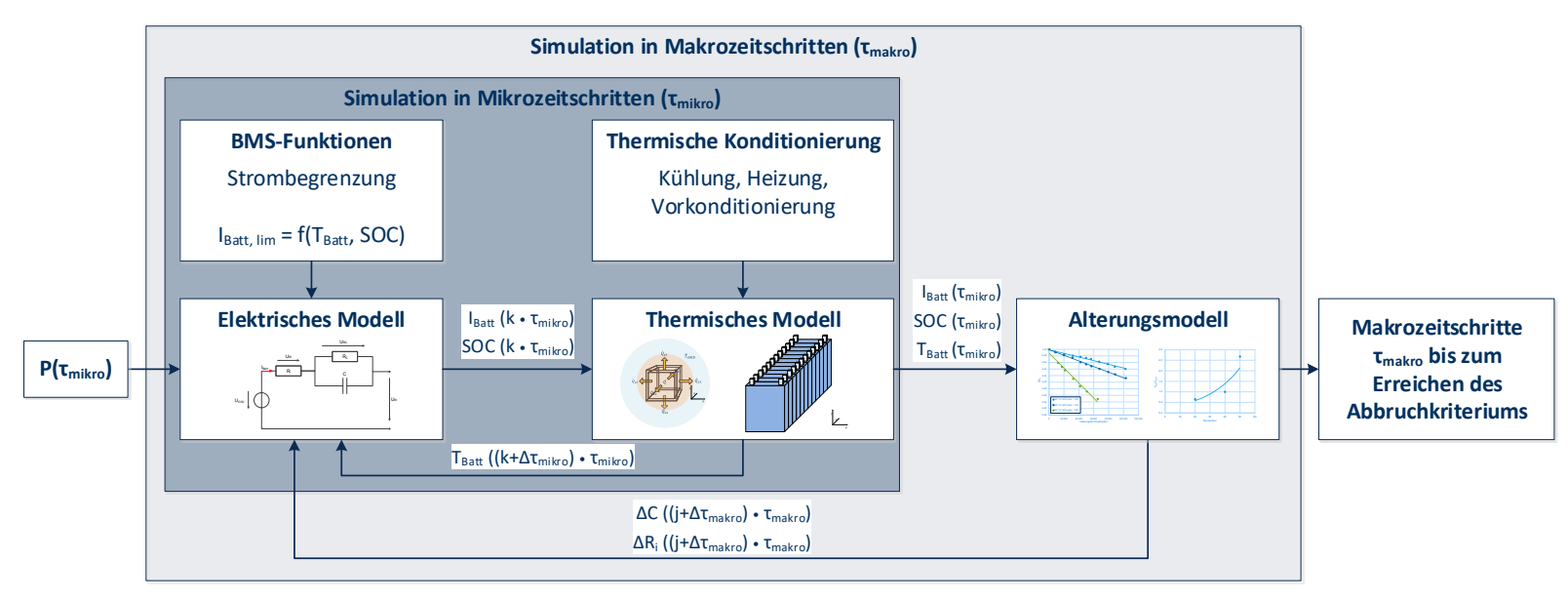

Abbildung 3-1: Batteriemodell als Simulationskern
\[ j, k \in \mathbb{N}\{1,2,3, \ldots\} \] 
Die Batteriesimulation wird im Zeitschrittverfahren durchgeführt. Aufgrund unterschiedlicher Zeitkonstanten der einzelnen Teilmodelle wird die Berechnung im Simulationskern auf zwei verschiedenen Zeitschrittebenen aufgeteilt. Trotz der Tatsache, dass thermische Größen im Allgemeinen größere Zeitkonstanten aufweisen als elektrische Größen, wird für die elektro-thermische Simulation eine Mikrozeitschrittebene gewählt. Dies ermöglicht die Berücksichtigung thermisch bedingter Effekte im elektrischen Modell durch eine Rückführung der Ergebnisse. Der Alterungsprozess weist hingegen eine deutlich größere Zeitkonstante auf als die elektro-thermischen Größen. Deshalb wird für die Alterungssimulation eine Makrozeitschrittebene gewählt. Die Schrittweite $\Delta \tau_{\text {mikro }}$ im Mikrozeitschrittbereich liegt üblicherweise im Bereich von $0,5 \mathrm{~s}$ bis $1,0 \mathrm{~s}$, wobei an dieser Stelle beliebige Schrittweiten gewählt werden können. Für die Makrozeitschrittebene $\tau_{\text {makro }}$ gilt im Rahmen dieser Arbeit $\Delta \tau_{\text {makro }}=1 d$ als kleinste Schrittweite. Zugunsten einer kürzeren Simulationsdauer können auch größere Makrozeitschrittweiten gewählt werden. Jedoch gilt es zu beachten, dass damit die Ergebnisgenauigkeit der Alterungssimulation beeinflusst wird, wie im weiteren Verlauf der Arbeit noch gezeigt wird (Vgl. Kapitel 5.2.1).

Die Eingangsgröße des Batteriemodells ist ein elektrischer Fahrzeugleistungsverlauf in der Auflösung der Mikrozeitschrittweite. Dieser Leistungsverlauf muss von der Batterie umgesetzt werden. Dafür werden im ersten Schritt zunächst die elektrischen Größen Batteriestrom, SOC, Ruhespannung und Klemmenspannung berechnet. Der Batteriestrom und der SOC werden an das thermische Modell übergeben und auf deren Basis die aktuelle Batterietemperatur berechnet. Einige Parameter des elektrischen Modells weisen eine Temperaturabhängigkeit auf (Vgl. Kapitel 4.1.3). Es ist somit erforderlich, dass die berechnete Batterietemperatur in das elektrische Modell zurückgeführt wird, sodass der folgende Zeitschritt mit angepassten elektrischen Parametern berechnet werden kann. Nach Abschluss der elektro-thermischen Simulation werden der resultierende SOC-, Temperatur- und Stromverlauf an das Alterungsmodell übergeben. Alle drei Größen bewirken in unterschiedlichem Maße eine Schädigung der Batterie, welche sich in Form einer Verringerung der nutzbaren Kapazität und eines erhöhten Innenwiderstands ausdrückt (Vgl. Kapitel 4.1.5). Diese Schädigung wird an das elektrische Modell zurückgegeben und die elektro-thermische Simulation mit angepasster Parametrierung des elektrischen Modells erneut durchgeführt. Die gesamte Simulation wird beendet, wenn die Schädigung der Batterie einen definierten Wert übersteigt und damit das Abbruchkriterium erreicht ist.

\subsection{Definition wesentlicher Randbedingungen zur Systemsimulation}

Zusätzlich zu den definierten Freiheitsgraden werden für die Systemsimulation und Optimierung einer Elektrobuslinie verschiedene Randbedingungen benötigt. Grundsätzlich werden diese für einen vollständigen Simulations- bzw. Optimierungsdurchlauf 
definiert. Geänderte Randbedingungen erfordern stets einen erneuten Berechnungsdurchlauf. Im Folgenden werden die für diese Arbeit wesentlichen Randbedingungen für die Systembestandteile Traktionsbatterie und Nachladung erläutert.

\subsubsection{Traktionsbatterie}

Für die Dimensionierung einer Traktionsbatterie sind neben der Kapazität, welche in Kapitel 3.1 als Freiheitsgrad definiert wurde, weitere Parameter zu berücksichtigen. Die wichtigsten sind im Folgenden aufgelistet und werden anschließend erläutert:

- Nennspannung und Betriebsspannungsbereich (bisher als Spannungslage bezeichnet),

- Zellchemie (z.B. NCA, NMC, $\left.\mathrm{LiFePO}_{4}\right)^{8}$,

- Bauform der Zellen (Rundzelle, prismatische Zelle, Pouchzelle),

- Geometrie der Zellanordnung in einem Batteriemodul bzw. einem Batteriesystem,

- Thermische (Vor-)Konditionierung (Heizen und/oder Kühlen).

Zur Ermittlung des Batterieenergieinhalts sind neben dem Freiheitsgrad der Kapazität die Nennspannung und der Betriebsspannungsbereich zu definieren. In den vergangenen Jahren hat sich bei Elektrobussen ein Betriebsspannungsbereich zwischen $600 \mathrm{~V}$ und $800 \mathrm{~V}$ als Quasistandard etabliert. ${ }^{9}$ Bei niedrigeren Spannungen verringert sich die Energieeffizienz aufgrund der steigenden Strombelastung unter Annahme einer konstanten Leistungsanforderung. Zusätzlich müssen bei hohen Strömen die betroffenen Betriebsmittel aufgrund der thermischen Beanspruchung entsprechend robuster und damit größer dimensioniert werden. Bei Wahl einer höheren Spannungslage (über 1000 V) müssen erhöhte Sicherheitsstandards hinsichtlich der Isolation angewendet werden, was aus Kostengründen oftmals vermieden wird. Die Nennspannung der Batterie resultiert aus der Wahl der Zellchemie und der Anzahl in Reihe geschalteter Zellen. Damit ergibt sich auch der Betriebsspannungsbereich, da alle Zellen einen definierten Spannungsbereich haben, in dem diese sicher und zuverlässig betrieben werden können. Dieser wird vom Zellhersteller im Datenblatt angegeben. Bei der Modellierung der Batterie ist der Betriebsspannungsbereich der Einzelzellen somit von entscheidender Bedeutung. In der Systemsimulation können beliebige Zellchemien mit ihren jeweiligen Spannungsbereichen parametriert und zu einem Batteriesystem konfiguriert werden.

\footnotetext{
${ }^{8}$ Die verschiedene Zellchemien sind in Abbildung 4-2 auf Seite 30 vergleichend dargestellt.

${ }^{9}$ Diese Aussage kann aus den Ausführungen in (Knote et al., 2017) und (UITP, 2017) abgeleitet werden.
} 
Die Bauform der Einzelzellen ist vor allem aus thermischer wichtig. So hat beispielsweise eine Pouchzelle eine andere thermische Charakteristik als eine prismatische Zelle oder eine Rundzelle (Fleckenstein, 2013). Die Bauform der Zellen ist als Randbedingung in der Systemsimulation zu berücksichtigen. Zur Bestimmung der Batterietemperatur ist neben den thermischen Eigenschaften der Einzelzelle auch der Aufbau der Batteriemodule entscheidend, welche später zu einem Batteriesystem konfiguriert werden. Je nach Aufbau können die einzelnen Module unterschiedliche thermische Charakteristiken aufweisen, welche in der Parametrierung der Systemsimulation entsprechend berücksichtigt werden.

Im realen Betrieb wird die Traktionsbatterie oftmals thermisch konditioniert. Dies kann sowohl Kühlen bei hohen Temperaturen als auch Heizen bei niedrigen Temperaturen bedeuten. Im weiteren Verlauf der Arbeit wird noch gezeigt, dass das Ziel der thermischen Konditionierung stets die Maximierung der Leistungsfähigkeit und die Minimierung der Schädigung der Batterie ist. Es kommen grundsätzlich drei verschiedene Kühlarten in Frage, welche in die Kategorien passive und aktive Kühlung unterschieden werden können:

- passive Luftkühlung (natürliche Konvektion),

- aktive Luftkühlung (forcierte Konvektion),

- aktive Flüssigkeitskühlung.

Zusätzlich zur thermischen Konditionierung im Fahrzeugbetrieb kann die Batterie bereits im Depot vorkonditioniert werden. Auch hier sind je nach Anwendungsfall eine Heizung bzw. Kühlung möglich. Wie im weiteren Verlauf der Arbeit noch gezeigt wird, sind die Kapazität und der Innenwiderstand von Li-Ionen Batterien und damit auch die verfügbare Reichweite und deren Leistungsfähigkeit temperaturabhängig. Zur Steigerung der Reichweite setzen viele Hersteller auf eine Heizung der Traktionsbatterie an kalten Tagen. Die dafür notwendige Energie wird aus der Batterie selbst bereitgestellt und hat somit wiederum eine negative Auswirkung auf die Reichweite. Dagegen muss die Batterie an heißen Tagen gekühlt werden, um eine überproportionale Schädigung aufgrund zu hoher Temperaturen zu vermeiden. Ziel der Vorkonditionierung ist es, die jeweilige Zieltemperatur der Batterie bereits vor Fahrtantritt einzustellen. Dabei wird die dafür notwendige Energie aus dem Netz und nicht aus der Batterie bezogen, was wiederum eine positive Auswirkung auf die Reichweite hat (Freitag, 2018b).

\subsubsection{Nachladung}

Neben den Freiheitsgraden Ladeleistung sowie Anzahl und Position der Ladepunkte sind die Technologie der Nachladung (konduktiv oder induktiv) sowie die Ladeleistung 
im Depot zu berücksichtigen. In Kapitel 2.1 wurden die zwei Ladetechnologien erläutert, welche für die Ladung von Elektrobussen in Frage kommen. Die unterschiedlichen Charakteristika der Energieübertragung können in der Systemsimulation berücksichtigt werden. Dies betrifft vor allem die maximal realisierbare Ladeleistung sowie den Wirkungsgrad der Übertragung. Eine detaillierte Erläuterung folgt in Kapitel 4.2.

Neben der Ladetechnologie ist die Nachladung im Depot eine Randbedingung in der Systemsimulation, wobei die Ladeleistung parametriert werden kann. Dabei ist stets nur eine feste Leistung wählbar, mit welcher alle Fahrzeuge gleichermaßen geladen werden. Im Rahmen dieser Arbeit wird lediglich untersucht, ob die parametrierte Ladeleistung ausreichend ist, um die Fahrzeuge auf den gewünschten Start-SOC zu laden. Eine gleichzeitige Ladung vieler Fahrzeuge in einem Depot kann dabei zu großen Leistungsanforderungen und Lastspitzen im speisenden Energienetz führen. Zur Reduzierung werden sog. Lastmanagementsysteme eingesetzt, welche über verschiedene Laderegime eine zeitliche Vergleichmäßigung der Leistungsanforderung bewirken können. Ein solches Lastmanagementsystem wurde beispielsweise in (Freitag, 2018a) entwickelt und in (Saidani, 2019) optimiert.

\subsection{Konzept einer mehrdimensionalen Optimierung mittels Parametervariation}

Gemäß den Forschungszielen dieser Arbeit soll die zu entwickelnde Simulationsumgebung eine integrierte Optimierungsfunktion beinhalten. Dafür werden die in Kapitel 3.1 hergeleiteten Freiheitsgrade des Systems als Variationsparameter definiert, welche innerhalb ihres festgelegten Wertebereiches beliebig miteinander kombinierbar sind. Es stellen jedoch nicht alle Kombinationen gültige Lösungen unter den gegebenen Bedingungen dar. Von den drei Hauptbestandteilen des Systems (Vgl. Abbildung 2-2) wird der Betrieb nicht als Variationsgröße, sondern geht als Randbedingung in der Optimierung ein. In Abbildung 3-1 ist das Alterungsmodell als eines von drei Teilen des Batteriemodells aufgeführt. Die Alterung der Batterie stellt in der Optimierung nur eine Berechnungsgröße dar, welche jedoch über das in Kapitel 4.3 zu entwickelnde Kostenmodell indirekt in der Systemoptimierung berücksichtigt wird. Das grundsätzliche Prinzip der Optimierung ist in Abbildung 3-2 gegeben.

Sowohl für die drei Variationsparameter als auch für die Berechnungsgröße der Batteriealterung kann jeweils ein separates Optimierungsziel formuliert werden. Diese sind in Tabelle 3-1 zusammengefasst. Daraus geht hervor, dass die Optimierung mehrere Dimensionen und Kriterien sowie konkurrierende Ziele aufweist. Allen gemein ist dabei die Minimierung der jeweils anfallenden Kosten. Die mehrkriterielle Optimierung wird über ein zu entwickelndes Kostenmodell auf ein Kriterium reduziert. Das Optimierungsziel dieser Arbeit ist die Minimierung der resultierenden Gesamtkosten der einzelnen Variationsparameter und Berechnungsgrößen. 


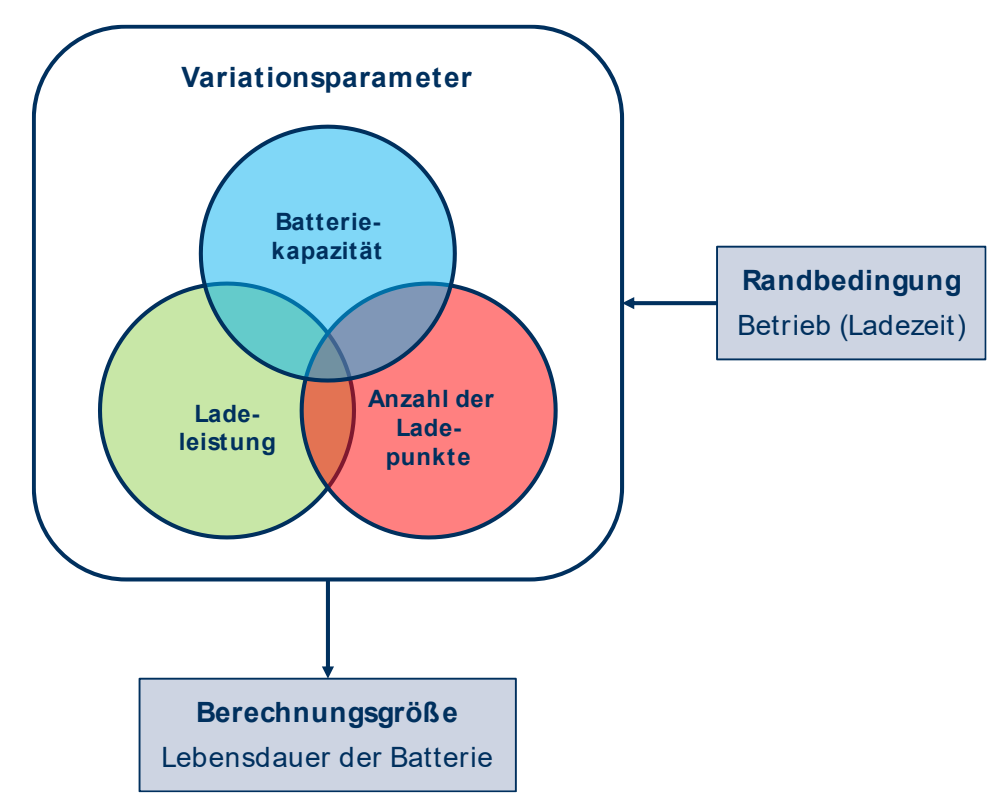

Abbildung 3-2: Prinzipdarstellung der Optimierung

Tabelle 3-1: Optimierungsziele einzelner Systemparameter und Berechnungsgrößen

\begin{tabular}{|c|c|c|}
\hline & Optimierungsziel & Begründung \\
\hline $\begin{array}{l}\text { Energieinhalt der } \\
\text { Batterie }\end{array}$ & Minimierung & $\begin{array}{l}\text { - geringes Gewicht und damit hohe } \\
\text { Energieeffizienz und Fahrgast- } \\
\text { kapazität } \\
\text { - geringe investive Kosten }\end{array}$ \\
\hline $\begin{array}{l}\text { Anzahl der } \\
\text { Ladepunkte }\end{array}$ & Minimierung & $\begin{array}{l}\text { - geringe investive Kosten } \\
\text { - geringer Planungsaufwand }\end{array}$ \\
\hline Ladeleistung & Minimierung & $\begin{array}{l}\text { - geringe investive Kosten } \\
\text { - niedrige energetische Verluste }\end{array}$ \\
\hline $\begin{array}{l}\text { Lebensdauer der } \\
\text { Batterie }\end{array}$ & Maximierung & $\begin{array}{l}\text { - geringe Folgekosten für Batterie- } \\
\text { tausch }\end{array}$ \\
\hline
\end{tabular}

In der Optimierung werden die einzelnen Parameterkombinationen gemäß Abbildung 3-2 automatisiert erstellt, simuliert und anschließend hinsichtlich wesentlicher Kostenbestandteile bewertet. Zur Bewertung und zum Vergleich der ermittelten Systemkonfigurationen wird ein Kostenmodell entwickelt. Die resultierenden Kosten dienen damit als Optimierungsziel, wobei minimale Kosten das Optimum darstellen. Das 
Modell zielt nicht darauf ab, eine klassische Total-Cost-of-Ownership-Berechnung durchzuführen, wie es beispielsweise in (Berthold, 2019; Kunith, 2017) vorgenommen wird (Vgl. Stand der Forschung, Kapitel 2.2). Vielmehr sollen an dieser Stelle lediglich die einzelnen technischen Konfigurationen auf Basis einer Differenzkostenbetrachtung untereinander vergleichbar gemacht werden. Demzufolge wird in dieser Arbeit ein einfaches Kostenmodell erstellt, welches lediglich die Hauptkostenbestandteile eines Elektrobussystems beinhaltet, in denen sich die betrachteten Systemkonfigurationen unterscheiden. Es wird an dieser Stelle die Bezeichnung Systemkosten eingeführt und im weiteren Verlauf der Arbeit verwendet.

Der Nennenergieinhalt einer Batterie ergibt sich aus der Nennspannung sowie der Kapazität. Beide Größen sind über eine Reihen- bzw. Parallelschaltung skalierbar. In Kapitel 3.3 wurde die Spannungslage der Batterie als Randbedingung definiert. Somit steht als variable Größe für den Energieinhalt der Batterie noch die Kapazität zur Verfügung. Diese wird im Rahmen dieser Arbeit als Variationsparameter ausgeführt. Um die Berechnungsergebnisse der Optimierung möglichst realitätsnah darzustellen, werden nur realistische Abstufungen der Variationsparameter betrachtet. Zur Variation der Kapazität der Batterie wird aufgrund der festen Spannungslage die Anzahl an parallelen Zellen variiert. Dafür kommen technisch bedingt nur ganzzahlige Werte in Frage. Unter Verwendung der in dieser Arbeit verwendeten Zellen (Vgl. Kapitel 4.1.3) ergibt sich demnach eine Schrittweite von ca. $15 \mathrm{kWh}$. Für die Betrachtung der Anzahl und Position der Ladepunkte muss auch deren Kombination berücksichtigt werden. Für jeden möglichen Ladepunktstandort können genau zwei Zustände definiert werden: Ladepunkt existiert (1) und Ladepunkt existiert nicht (0). Die Kombinationsmöglichkeiten $Z$ ergeben sich in Abhängigkeit der Anzahl $n$ potentieller Ladepunkte wie folgt:

$$
Z=2^{n}
$$

Tabelle 3-2: Ladepunktkombinationen für 2 Ladepunkte

\begin{tabular}{cccc}
\hline ID & Depot & Ladepunkt 1 & Ladepunkt 2 \\
\hline$(0 / 0)$ & 1 & 0 & 0 \\
$(0 / 1)$ & 1 & 0 & 1 \\
$(1 / 0)$ & 1 & 1 & 0 \\
$(1 / 1)$ & 1 & 1 & 1 \\
\hline
\end{tabular}

Für eine Linie mit $n=2$ möglichen Ladepunkten entlang der Strecke (beispielweise an den Wendepunkten) ergeben sich demnach $Z=4$ mögliche Kombinationen gemäß Formel (3-2), welche in Tabelle 3-2 gegeben sind. Im weiteren Verlauf dieser Arbeit wird stets eine ID in der Form aus Spalte 1 für die Beschreibung einer konkreten 
Ladepunktkombination verwendet. Die Depotladung wird dabei nicht variiert, da im Rahmen dieser Arbeit gilt, dass die Fahrzeuge unabhängig von den Ladepunkten entlang der Strecke immer im Depot geladen werden. Die erste Kombination (0/0) entspricht demnach einer Ausführung als Depot-Charger, während die übrigen Varianten Opportunity-Charger darstellen. Es wird deutlich, dass analog zu den anderen Variationsparametern auch die Kombination der Ladepunkte nur eine sehr geringe Variationsbreite hat.

Für die Ladeleistung ergibt sich im Unterschied zu den anderen Variationsparametern keine Einschränkung in der Schrittweite, d.h. es können beliebig kleine Abstufungen gewählt werden. Es wird dennoch auch hier eine möglichst realitätsnahe Anwendung bevorzugt, da Hersteller und Lieferanten von Ladesystemen meist fertige und skalierbare Produkte anbieten. In dieser Arbeit wird deshalb eine Schrittweite von $10 \mathrm{~kW}$ gewählt.

Aus diesen Einschränkungen der Schrittweite der einzelnen Variationsparameter ergibt sich bei deren Kombination eine vergleichsweise kleine Anzahl an möglichen Kombinationen. In Tabelle 3-3 sind beispielhaft realitätsnahe Wertebereiche inkl. der definierten Schrittweiten angegeben. Es handelt sich hierbei um einen vergleichsweise kleinen Parameterraum, wenn dieser in Relation zu anderen Optimierungsproblemen gestellt wird. Als Beispiel sei die Ermittlung einer Strategie zur energieeffizienten Längsführung von Hybridfahrzeugen herangezogen, bei der wie in (Uebel, 2018), bedingt durch eine sehr große Anzahl an Variationsparametern und einem zeit- bzw. wegkontinuierlichem Prozess, nahezu unendlich viele Kombinationsmöglichkeiten existieren.

Tabelle 3-3: Beispiel des Wertebereichs und Schrittweiten der Variationsparameter

\begin{tabular}{lccc}
\hline Variationsparameter & Wertebereich & Schrittweite & $\begin{array}{c}\text { Anzahl } \\
\text { Schritte }\end{array}$ \\
\hline $\begin{array}{l}\text { Energieinhalt Batterie } \\
\begin{array}{l}\text { Anzahl Ladepunkt- } \\
\text { kombination }\end{array}\end{array}$ & $40 \mathrm{kWh}-400 \mathrm{kWh}$ & $15 \mathrm{kWh}$ & 24 \\
Ladeleistung & $50 \mathrm{~kW}-500 \mathrm{~kW}$ & $10 \mathrm{~kW}$ & 46 \\
\hline
\end{tabular}

Im gezeigten Beispiel in Tabelle 3-3 ergeben sich 4416 Kombinationsmöglichkeiten. Es wird in dieser Arbeit der Ansatz gewählt, alle Kombinationen des Parameterraums zu berechnen und auf einen Einsatz eines speziellen Optimierungsalgorithmus zu verzichten. Diese haben generell das Ziel, die Anzahl zu berechnender Kombinationen zugunsten einen kürzeren Berechnungszeit zu verringern und dabei dennoch die optimale Lösung zu finden. Aufgrund der kleinen Anzahl an Kombinationen wird die 
Rechendauer jedoch als unkritisch betrachtet. Die Berechnung aller möglichen Parameterkombinationen eines Optimierungsraums wird auch als Brute-ForceMethode bezeichnet.

\subsection{Entwicklung des Frameworks zur Systemsimulation und Optimierung}

Auf Basis der bisherigen Ausführungen in diesem Kapitel wird nun ein detaillierter Programmablaufplan entwickelt. Dieser ist in Abbildung 3-3 dargestellt. Aus dem Wertebereich der Variationsparameter wird zunächst eine konkrete Systemkonfiguration definiert. Für diese wird unter Annahme einer maximal gealterten Batterie die technische Machbarkeit für die gegebene Strecke ermittelt. Eine maximal gealterte Batterie wird im Rahmen dieser Arbeit wie folgt definiert ${ }^{10}$ :

- $80 \%$ Restkapazität (in Bezug auf 100\% Nennkapazität),

- $200 \%$ Innenwiderstand (in Bezug auf $100 \%$ des initialen Wertes).

Basis der Berechnung ist stets ein elektrischer Fahrzeugleistungsverlauf, welcher als Eingang in den Berechnungskern definiert wurde (Vgl. Kapitel 3.2, Abbildung 3-1) ${ }^{11}$. Für die Berechnung mit maximal gealterter Batterie wird zunächst die elektro-thermische Belastung der Batterie ermittelt. Anschließend wird überprüft, ob die als Randbedingung definierte Depotladeleistung ausreichend ist, um die Fahrzeuge innerhalb der zur Verfügung stehenden Depotladezeit wieder auf den definierten Start-SOC aufzuladen. Die Depotladezeit ergibt sich aus der Differenz der täglichen Betriebszeit und 24 Stunden eines Tages. Sowohl die Überschreitung der elektro-thermischen Belastung als auch eine nicht ausreichende Nachladung führen dazu, dass die gewählte Konfiguration als technisch nicht realisierbar eingestuft und damit ausgeschlossen wird. In diesem Fall wird die Berechnung abgebrochen und eine neue Systemkonfiguration definiert.

Ist der betrachtete Leistungsverlauf mit der gewählten Systemkonfiguration technisch realisierbar, so wird anschließend die Batteriealterung berechnet. Dabei wird zunächst für eine neue Batterie die elektro-thermische Belastung auf Mikrozeitschrittebene berechnet. Anschließend wird die Ladung im Depot simuliert. Dies dient der Vervollständigung der Batteriebelastung, da anschließend in den Funktionen Zyklusanalyse und Alterungsmodell (Vgl. Abbildung 3-3) die resultierende Schädigung der Batterie auf Basis von Makrozeitschritten berechnet wird. Diese wird an das elektrische Modell

\footnotetext{
${ }^{10}$ Diese Werte werden typischerweise für Elektrofahrzeuge und Hybridfahrzeuge verwendet (Kampker et al., 2018).

${ }^{11}$ Die Ermittlung des Fahrzeugleistungsbedarfs ist nicht Betrachtungsgegenstand dieser Arbeit (Vgl. Kapitel 2.3).
} 
zurückgegeben und die elektro-thermische Belastung erneut berechnet. Der elektrische Leistungsverlauf des Fahrzeugs bleibt dabei jedoch unverändert, da dieser unabhängig von der Batterieschädigung umgesetzt werden muss. Dadurch bedingt steigt die elektro-thermische Belastung der Batterie mit zunehmender Alterung an. Diese Schleife wird so lange durchlaufen, bis eines der oben definierten Abbruchkriterien einer maximal gealterten Batterie erreicht wird. Abschließend erfolgt die ökonomische Bewertung der Systemkonfiguration anhand eines Kostenmodells. Der Programmablauf wird nun für die nächste Konfiguration wiederholt, bis alle Kombinationsmöglichkeiten der Variationsparameter abgearbeitet sind. Die Konfiguration mit den minimalen Kosten stellt für die betrachtete Linie das Optimum dar. 
3 | Konzept zur simulationsbasierten Systemauslegung einer Elektrobuslinie

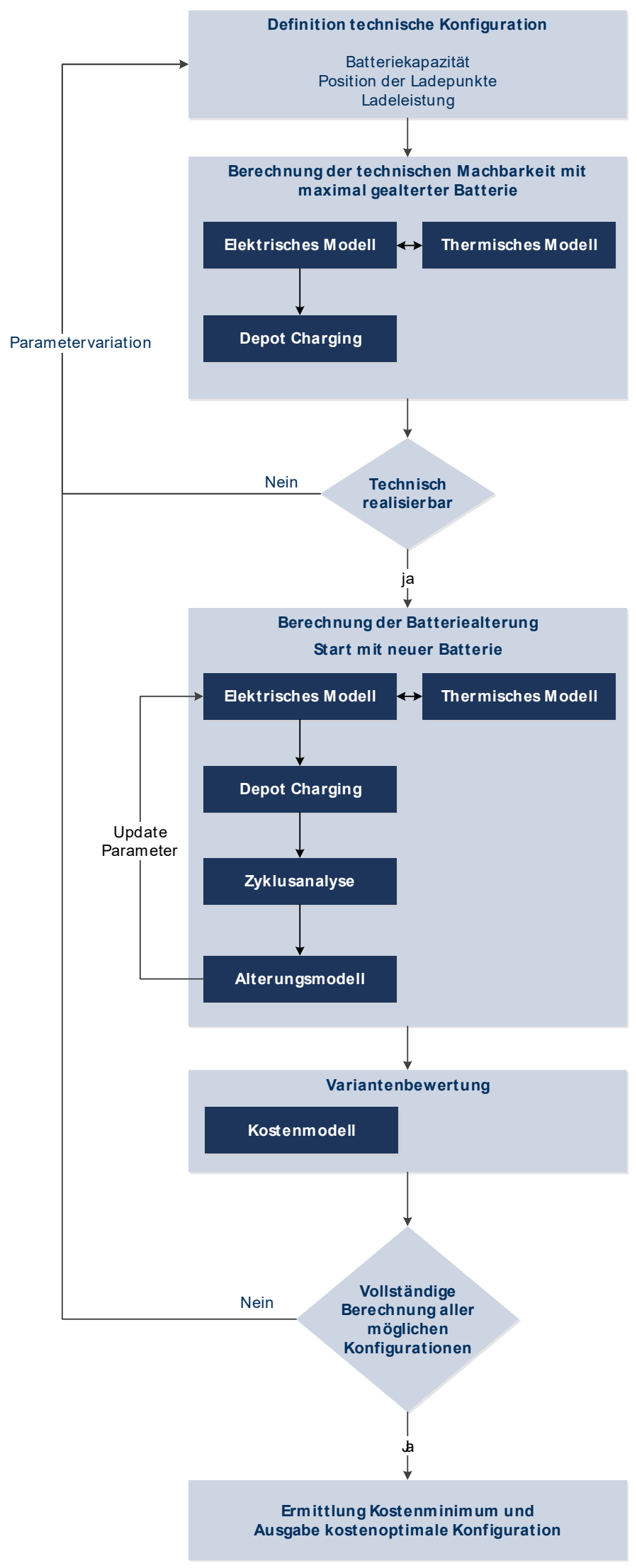

Abbildung 3-3: Globaler Programmablaufplan 



\section{$4 \quad$ Modellbildung und Modellvalidierung}

In diesem Kapitel werden die einzelnen Modellbestandteile und Funktionen des Simulationskerns gemäß Abbildung 3-1 und Abbildung 3-3 modelliert, parametriert und validiert. Dies umfasst die folgenden Modelle:

- Batteriemodell mit den Teilmodellen

- Elektrisches Modell,

- Thermisches Modell,

- Alterungsmodell,

- Modell der Ladepunkte,

- Kostenmodell.

\subsection{Batteriemodell}

\subsubsection{Definition der Modellierungs- und Betrachtungsebenen}

Traktionsbatterien sind gewöhnlich mehrschichtig aufgebaut. Die kleinste Einheit stellen Einzelzellen dar, welche dann zu Modulen zusammengesetzt werden. Ein vollständiges Batteriesystem besteht aus einer Konfiguration mehrerer Module, welche beliebig seriell und parallel geschaltet werden können. Der grundsätzliche Aufbau eines solchen Systems ist beispielhaft in Anhang D.1 dargestellt und beschrieben.

Tabelle 4-1: $\quad$ Übersicht Batteriemodellbildung und Validierung in Kapitel 4.1

\begin{tabular}{|c|c|c|c|}
\hline & $\begin{array}{c}\text { elektrisches } \\
\text { Modell } \\
\text { Kap. 4.1.3 }\end{array}$ & $\begin{array}{c}\text { thermisches } \\
\text { Modell } \\
\text { Kap. 4.1.4 }\end{array}$ & $\begin{array}{c}\text { Alterungs- } \\
\text { modell } \\
\text { Kap. 4.1.5 }\end{array}$ \\
\hline $\begin{array}{l}\text { Ebene der Modell- } \\
\text { bildung und } \\
\text { Parametrierung }\end{array}$ & Zellebene & Zellebene & Zellebene \\
\hline $\begin{array}{l}\text { Skalierung zur } \\
\text { Systemsimulation }\end{array}$ & Batterieebene & $\begin{array}{l}\text { Modulebene } \\
\text { (Batterieebene) }\end{array}$ & -- \\
\hline Ebene der Validierung & Zellebene & $\begin{array}{l}\text { Zellebene und } \\
\text { Batterieebene }\end{array}$ & Zellebene \\
\hline
\end{tabular}


Alle Teilmodelle werden zunächst auf Zellebene entwickelt und parametriert. Anschließende findet eine Skalierung einiger Modell statt, um diese in der Systemsimulation möglichst realitätsnah zu berücksichtigen. Demzufolge können die Modellierungsebene und die Validierungsebene unterschiedlich sein. Die folgenden Aussagen sind in Tabelle 4-1 zusammengefasst.

- Das elektrische Teilmodell wird über die bekannten Zusammenhänge zur Parallel- und Reihenschaltung elektrischer Größen auf Batterieebene skaliert. Das elektrische Verhalten der Batterie wird jedoch hauptsächlich von den Einzelzellen charakterisiert, weshalb die Validierung auf Zellebene stattfindet.

- Das thermische Teilmodell wird von der Zellebene auf die Modulebene skaliert. Hierbei ist die räumliche Anordnung der Zellen zueinander von entscheidender Bedeutung und somit Bestandteil der Modellbildung und Skalierung. Es wird im Rahmen dieser Arbeit vereinfachend davon ausgegangen, dass zwischen den einzelnen Modulen eines Batteriesystems keine thermische Interaktion besteht, sodass aus thermischer Sicht die Modulebene der Batterieebene entspricht. Die Validierung erfolgt deshalb sowohl auf Zellebene als auch auf Batterieebene.

- Das Alterungsmodell wird auf Zellebene entwickelt und validiert. Im Gegensatz zu den vorherigen Teilmodellen ist zur Beurteilung der Ergebnisse keine Skalierung auf Modul- oder Batterieebene erforderlich.

\subsubsection{Modellbildung, Funktionsprinzip und Kenngrößen von Li-Ionen Zellen}

Es bestehen verschiedene Möglichkeiten zur Modellbildung von Batteriesystemen, welche sich hinsichtlich Modellierungsaufwand und Ergebnisgranularität unterscheiden. In (Jossen, 2016) wird unterschieden in:

- Bilanzierende Modelle (BM),

- Ersatzschaltbildmodelle (ESB),

- Fundamentale Modelle (FM).

In Tabelle 4-2 sind die genannten Modelle miteinander verglichen. Die Wahl des Modells, welches im Rahmen dieser Arbeit implementiert wird, kann aus den Anforderungen aus Kapitel 2.2 abgeleitet werden. Demnach sollen eine Simulation des elektro-thermischen Verhaltens der Batterie und der resultierenden Alterung durchgeführt werden. Dafür muss u.a. die Batterietemperatur bekannt sein, wofür wiederum der Batteriestrom eine notwendige Eingangsgröße ist. Daraus resultiert, dass bilanzierende Modelle an dieser Stelle nicht verwendet werden können. Bei der Wahl zwischen ESB und FM soll vor allem die Rechenzeit als Entscheidungsgrundlage berücksichtigt werden. Aufgrund der langen Rechenzeiten sowie der hohen Komplexität 
wird auf die Anwendung eines FM verzichtet. Die Batterie wird in dieser Arbeit auf Basis eines ESB modelliert. Im Folgenden werden die Funktionsweise von Li-Ionen Batterien kurz erläutert sowie wichtige Kenngrößen eingeführt und definiert.

Tabelle 4-2: Vergleich von Modellen für Batteriesysteme, nach (Jossen, 2016)

\begin{tabular}{lccc}
\hline & BM & ESB & FM \\
\hline Komplexität & gering & mittel & hoch \\
Parametrierungsaufwand & gering & hoch & theor. gering \\
Dynamik & nur SOC & sehr detailliert & sehr detailliert \\
Änderung & sehr schnell & schnell & sehr langsam \\
Spannung/Strom für Zelle & nein & ja & ja \\
Spannung/Strom Ver- & nein & nein & ja \\
teilung innerhalb der Zelle & & & \\
\hline
\end{tabular}

Neben den meisten mobilen Consumer-Produkten wie Handys oder Notebooks werden Li-lonen Batterien bereits heute im großen Stil für die Elektrifizierung von Fahrzeugen eingesetzt. Dabei ist es unerheblich, ob es sich um rein batterie-elektrische Fahrzeuge oder solche mit hybriden Antriebssystemen handelt. Li-lonen Batterien haben im Vergleich zu anderen Batterietypen (wie beispielsweise NiMH oder Bleibatterien) eine deutlich höhere Energiedichte und eignen sich deshalb besonders für den Einsatz in mobilen Anwendungen. Des Weiteren zeichnen sich Li-Ionen Batterien durch eine vergleichsweise gute Langlebigkeit aus (Reif, 2012; Kurzweil et al., 2018).

Der Energiespeichervorgang in einer Li-lonen Zelle beruht auf einer reversiblen Einlagerung und Auslagerung von Li-lonen in die Wirtsgitter der Elektroden. Das Prinzip ist in Abbildung 4-1 veranschaulicht. Die Elektroden sind mit Hilfe eines Separators voneinander getrennt. Hauptaufgabe des Separators ist es, einen Kurzschluss der Zelle durch Kontakt der Elektroden zu verhindern. Gleichzeitig muss der Separator jedoch eine hinreichende Durchlässigkeit besitzen, damit die Li-lonen weitestgehend unbehindert passieren können (Richter, 2017). An der Grenze zwischen Anode und Elektrolyt bildet sich eine passive Schicht aus, die sog. "Solid Electrolyte Interphase" (SEI). Diese Schicht ist vor allem für das Verständnis der Alterung relevant und wird in Kapitel 4.1.5 erläutert. 


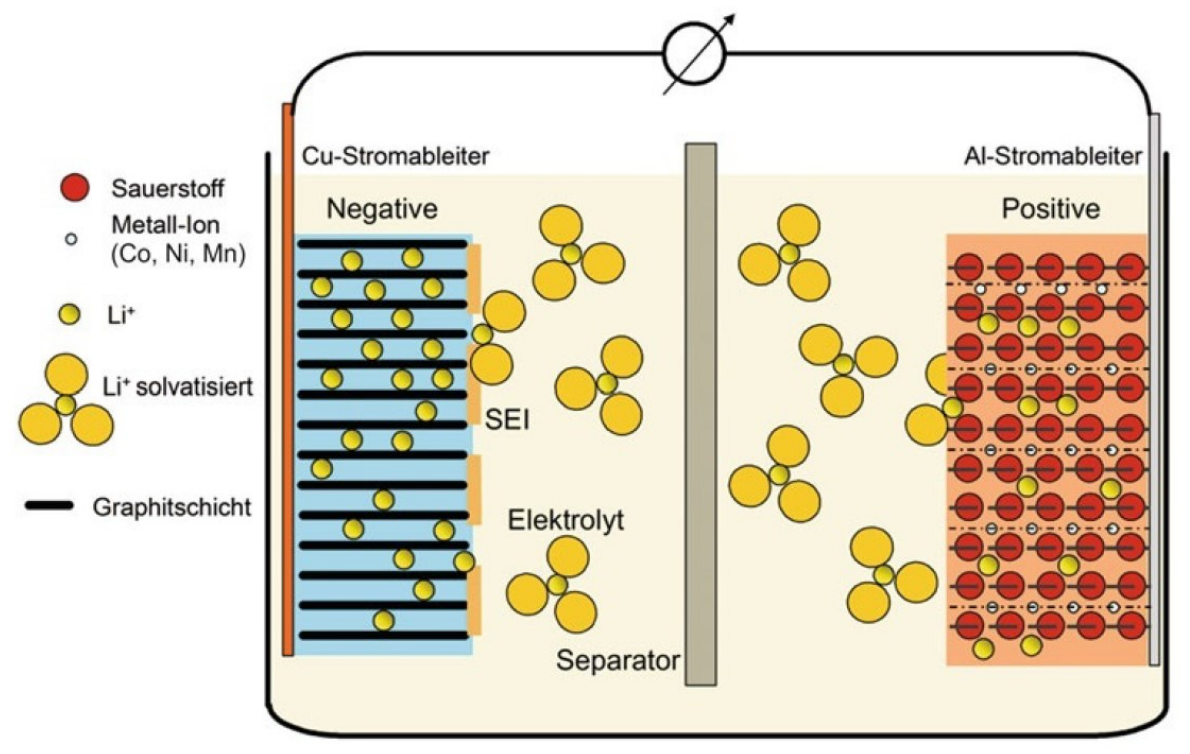

Abbildung 4-1: Funktionsprinzip einer Li-lonen Zelle (Reif, 2012)

Es existiert eine Vielzahl verschiedener Li-Ionen Batterietypen, welche meist nach den verwendeten Aktivmaterialien der Kathode benannt werden. Im PKW-Bereich wird zurzeit hauptsächlich auf die Kombination NMC (Nickel-Mangan-Kobalt) gesetzt, wobei einige Hersteller auch NCA-Kathoden einsetzen (Nickel-Kobalt-Aluminium) (Thielmann et al., 2015). Für Anwendungen in elektrifizierten Nutzfahrzeugen und Bussen wird oftmals auch Lithium-Eisenphosphat (LiFePO ${ }_{4}$ oder LFP) verwendet (Bloomberg, 2018). Eine Übersicht der aktuell in Fahrzeuganwendungen eingesetzten Kathodenmaterialien ist in Abbildung 4-2 gegeben. Daraus geht hervor, dass $\mathrm{LiFePO}_{4}$ Zellen eine vergleichsweise niedrige Energiedichte aufweisen, im Vergleich zu NCA oder NMC jedoch als sehr sicher eingestuft werden können. Ihr besonderer Vorteil liegt in der hohen Leistungsfähigkeit bei gleichzeitiger Langlebigkeit, weshalb sie für Busanwendungen mit hohen Leistungs- und Sicherheitsanforderungen besonders geeignet erscheinen. Im Folgenden werden wichtige Kenngrößen für den elektrischen Betrieb von Li-Ionen Batterien eingeführt und erläutert.

\section{Ruhespannung}

Die Ruhespannung $U_{\mathrm{OCV}}$ (Open Circuit Voltage - OCV) ist die Spannung einer Zelle, welche im unbelasteten Zustand und nach Abklingen aller internen Ausgleichsprozesse an den Klemmen gemessen werden kann. Bis zum vollständigen Abklingen dieser Prozesse können teilweise mehrere Stunden vergehen. Die Ruhespannung ist abhängig vom aktuellen Ladezustand und der Temperatur der Zelle (Jossen et al., 2019). Eine beispielhafte Ruhespannungskennlinie einer $\mathrm{LiFePO}_{4}$ Zelle ist in Abbildung 4-5 gegeben. Verfahren zur Ermittlung der Kennlinie werden in Kapitel 4.1.3 beschrieben. 

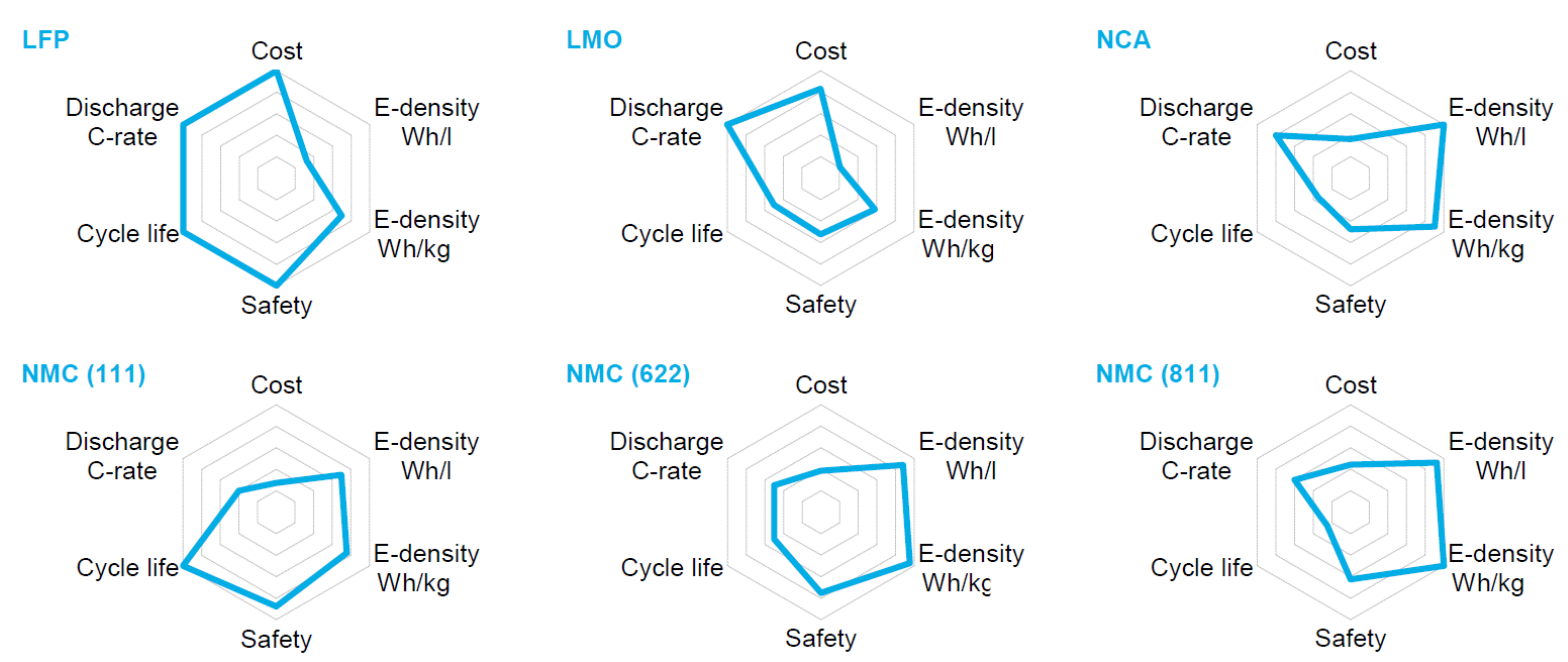

\section{Abbildung 4-2: Übersicht der aktuell in Fahrzeugen eingesetzten Kathodenmaterialien (Bloomberg, 2018)}

\section{Klemmenspannung}

Die Klemmenspannung $U_{\mathrm{Kl}}$ wird dann gemessen, wenn die Zelle belastet wird oder gerade belastet wurde. Sie berechnet sich aus der Ruhespannung und diversen Überspannungen, welche aus den chemischen Prozessen im Inneren der Zelle resultieren (Jossen et al., 2006). Dazu zählen:

- Überspannung aus ohmschem Spannungsfall,

- Überspannung durch Ladungsdurchtritt,

- Diffusionsüberspannung.

Die einzelnen Bestandteile können anhand eines Lastsprungs erläutert werden und sind in Abbildung 4-3 (oben) beispielhaft gezeigt. Die Vorzeichen der Überspannungen sind für den Lade- und Entladefall unterschiedlich und somit stromrichtungsabhängig. Im Falle einer Ladung ist die Klemmenspannung größer als die Ruhespannung, im Entladefall ist sie entsprechend kleiner.

\section{Lade- und Entladeschlussspannung}

Die Lade- und Entladeschlussspannung $U_{\max }$ und $U_{\min }$ stellen die Spannungsgrenzen einer Zelle dar und definieren somit den Betriebsbereich. Durch die Einhaltung der Werte wird ein sicherer und zuverlässiger Betrieb der Zelle garantiert. Während eines Ladevorgangs darf die Ladeschlussspannung $U_{\max }$ nicht überschritten werden, während einer Entladung gilt dies analog für die Entladeschlussspannung $U_{\min }$. Es gilt 
zu beachten, dass Li-lonen Zellen nicht überladesicher sind. Nachdem das Potential der Zellreaktion vollständig ausgeschöpft wurde, erfolgt eine Zersetzung des Elektrolyts, wodurch die Zelle nachhaltig und unmittelbar beschädigt wird (Reif, 2012). Eine Überwachung und Einhaltung der Spannungsgrenzen ist somit für ein Batteriemanagementsystem eine wichtige Funktion.

\section{Kapazität}

Die Kapazität einer Li-lonen Zelle beschreibt die elektrische Ladung $Q$, welche bei einem definierten Strom innerhalb der Spannungsgrenzen daraus entnommen werden kann (Kritzinger, 2018a).

$$
Q=\int_{t_{0}}^{t_{1}} i(t) d t
$$

Im Datenblatt einer Zelle ist meist die Nennkapazität $C_{\mathrm{N}}$ angegeben. Diese beschreibt die Entladung der Zelle unter Nennbedingungen innerhalb einer Stunde.

\section{Ladezustand}

Der Ladezustand einer Zelle (State of Charge - SOC) beschreibt das Verhältnis aus in der Zelle verfügbarer Kapazität $C_{\text {act }}$ und Nennkapazität $C_{\mathrm{N}}$.

$$
S O C=\frac{C_{\text {act }}}{C_{\mathrm{N}}}
$$

Gemäß Formel (4-2) entspricht eine vollgeladene Batterie einem SOC von 100\% und eine leere Batterie einem SOC von $0 \%$. Wie im weiteren Verlauf der Arbeit noch gezeigt wird, unterliegen Li-Ionen Batterie einem Alterungsprozess, bei welchem sich die Kapazität der Zellen im Laufe der Zeit reduziert. Zur Berechnung des tatsächlichen SOC muss die Nennkapazität demnach um die bis zu diesem Zeitpunkt aufgetretene Alterung korrigiert werden.

\section{C-Rate}

Als C-Rate wird der Zellstrom in Abhängigkeit der Nennkapazität bezeichnet.

$$
C-\text { Rate }=\frac{I_{\text {Zelle }}}{C_{\mathrm{N}}}
$$


Eine vollständige Entladung einer 20 Ah Zelle innerhalb einer Stunde mit 20 A entspricht einer C-Rate von $1 \mathrm{C}$. Die Skalierung des Stroms anhand der Kapazität ermöglicht einen Vergleich verschiedener Zellen mit unterschiedlichen Kapazitäten (Kritzinger, 2018a).

\subsubsection{Elektrisches Modell}

In diesem Abschnitt wird ein Modell zur Beschreibung des elektrischen Zellverhaltens hergeleitet. Die Parametrierung sowie alle späteren Simulationen und Optimierungsrechnungen basieren auf einer Lithium-Eisenphosphat Pouchzelle des Herstellers A123. Diese Zelle wurde u.a. im Pilotprojekt "Elektrobuslinie 79" in Dresden eingesetzt (Bunzel et al., 2016). In Tabelle 4-3 sind die wichtigsten Parameter angegeben. Das Modell ist jedoch derart konzipiert, dass beliebige Zellchemien parametriert und berechnet werden können.

Tabelle 4-3: Auszug aus dem Zelldatenblatt (A123 Systems, 2011, 2014)

\begin{tabular}{ll}
\hline \multicolumn{1}{c}{ Kenngröße } & \multicolumn{1}{c}{ Wert } \\
\hline Nennspannung & $3,3 \mathrm{~V}$ \\
Nennkapazität & $19,6 \mathrm{Ah}$ \\
Ladeschlussspannung & $3,6 \mathrm{~V}$ \\
Entladeschlussspannung & $2,0 \mathrm{~V}$ \\
Nennenergieinhalt & $65 \mathrm{Wh}$ \\
Abmessungen & $(7,25 \times 160 \times 227) \mathrm{mm}$ \\
Gewicht & $0,495 \mathrm{~kg}$ \\
Bauform & $\mathrm{Pouch}^{2}$ \\
Kathodenmaterial & $\mathrm{LiFePO}_{4}$ \\
\hline
\end{tabular}

Aus der Literatur sind verschiedene Modellierungstiefen für elektrische Modelle auf Basis von ESB bekannt (Einhorn et al., 2011). Allen gemein ist der Ansatz bestehend aus einer Ruhespannungsquelle und einem ohmschen Innenwiderstand. Daran anschließend können je nach gewünschter Modellgenauigkeit ein oder mehrere RCGlieder geschaltet werden. Die notwendigen Elemente des ESB können anhand der Spannungsantwort einer Li-lonen-Zelle auf einen Stromsprung gezeigt werden. Dabei werden drei markante Bereiche unterschieden: 
- Ohmscher Spannungsfall: keine zeitliche Verzögerung,

- Ladungsdurchtritt und Doppelschicht: geringe Zeitkonstante,

- Diffusion: große Zeitkonstante.

Es wird deutlich, dass der ohmsche Spannungsfall sowie der Spannungsfall aufgrund des Ladungsdurchtritts jeweils einen deutlich größeren Anteil am Gesamtspannungsfall haben als der Diffusionsspannungsfall. Für eine detaillierte Beschreibung und Erläuterung der hinter diesen Spannungsfällen liegenden chemischen Prozesse innerhalb der Zelle sei auf die einschlägige Fachliteratur verwiesen, beispielsweise (Jossen et al., 2019).

Der ohmsche Spannungsfall wird über einen Widerstand modelliert. Die übrigen beiden Spannungsfälle werden aufgrund ihrer Zeitkonstanten mittels einer Parallelschaltung aus jeweils einem Widerstand und einem Kondensator modelliert. Die Grundlage der elektrischen Zellmodellierung über eine Reihenschaltung von RCGliedern wird in (Buller, 2002) hergeleitet und ist seitdem Stand der Technik. In Abbildung 4-3 (unten) ist das ESB einer Li-lonen-Zelle mit 2 RC-Gliedern dargestellt. Da mit steigender Anzahl der RC-Glieder im Allgemeinen auch der Rechen- und Parametrierungsaufwand steigt (Jossen, 2016), werden in dieser Arbeit nur die Spannungsfälle des ohmschen Innenwiderstands und des Ladungsdurchtritts modelliert. Anhand einer Validierungsmessung und -simulation wird später gezeigt, dass damit eine ausreichend hohe Genauigkeit für die Betrachtungen im Rahmen dieser Arbeit erreicht wird.

Das in dieser Arbeit verwendete ESB einer Einzelzelle inkl. der definierten Ströme und Spannungen ist in Abbildung 4-4 dargestellt. Die Ersatzschaltung wird gemäß Tabelle 4-1 zunächst auf Zellebene parametriert und anschließend über die bekannten Zusammenhänge zur Reihen- und Parallelschaltung auf die Batterieebene skaliert, wodurch sich lediglich die Parametrierung, nicht jedoch der Aufbau des ESBs ändert. Folgende Bestandteile werden parametriert:

- OCV (Ruhespannung) $U_{\mathrm{OCV}}$

- Ohmscher Innenwiderstand $R_{\mathrm{i} \text {, }}$

- Kapazität $C$ und Widerstandswert $R_{\mathrm{C}}$ des RC-Glieds.

Die folgenden Modellgleichungen werden zum besseren Verständnis für die Batterieebene angegeben. Dies betrifft vor allem die Definition des Laststroms, welcher als Batteriestrom und nicht als Zellstrom verwendet wird. 


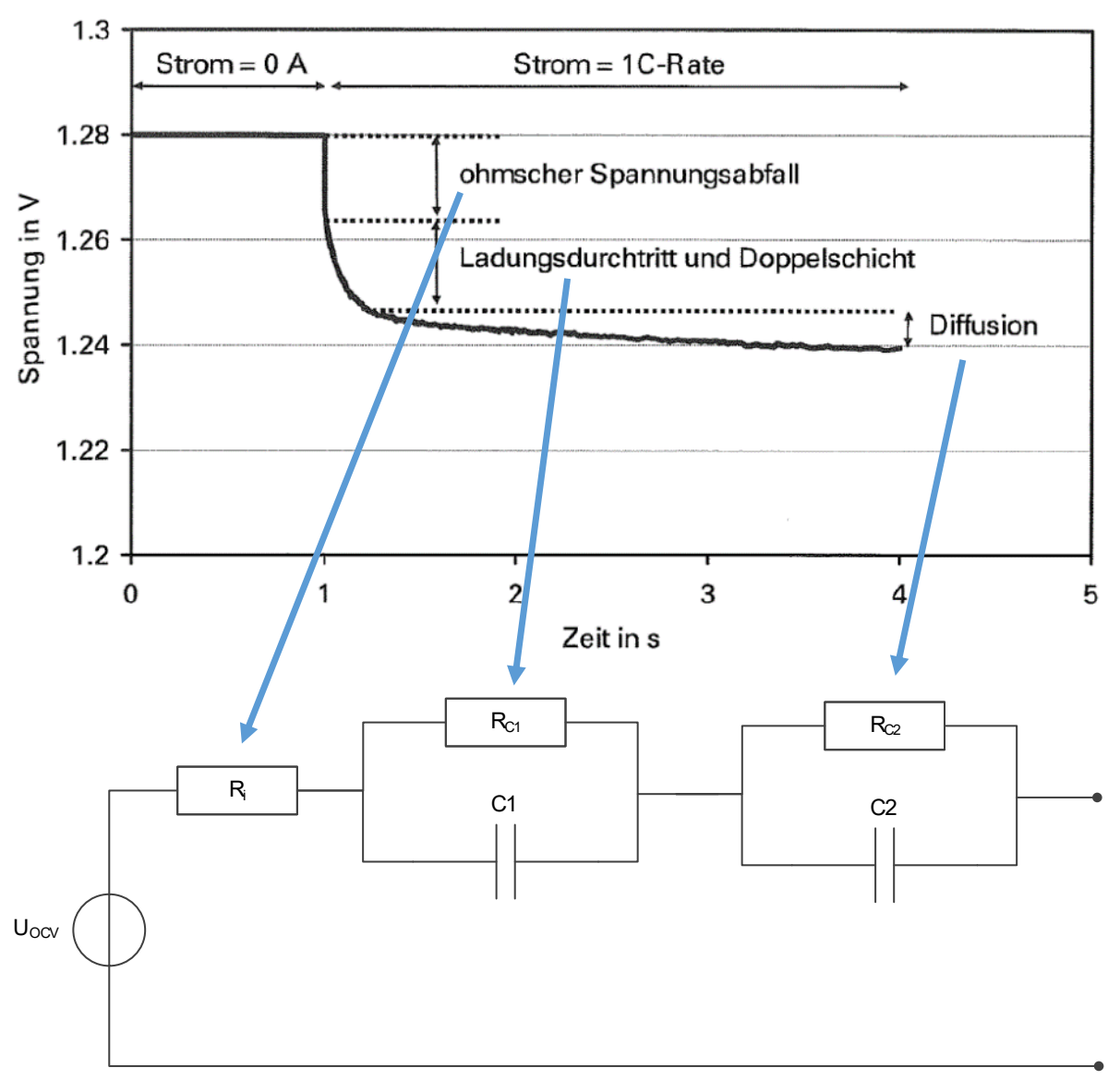

Abbildung 4-3: oben: Spannungsantwort einer Li-lonen Zelle auf einen Stromsprung, (Jossen et al., 2006), unten: Zuordnung der Spannungsfälle zum ESB

Anhand des ESB in Abbildung 4-4 kann die Spannungsgleichung des Modells hergeleitet werden.

$$
\begin{gathered}
0=U_{\mathrm{OCV}}-U_{\mathrm{Ri}}-U_{\mathrm{RC}}-U_{\mathrm{Kl}} \\
U_{\mathrm{Kl}}=U_{\mathrm{OCV}}-U_{\mathrm{Ri}}-U_{\mathrm{RC}}=U_{\mathrm{OCV}}-I_{\mathrm{Batt}} \cdot R_{\mathrm{i}}-I_{\mathrm{Batt}} \cdot R_{\mathrm{C}} \cdot\left(1-e^{\frac{-t}{C \cdot R_{C}}}\right)
\end{gathered}
$$

Die Leistung der Batterie wird wie folgt berechnet:

$$
P_{\mathrm{Batt}}=I_{\mathrm{Batt}} \cdot U_{\mathrm{Kl}}=I_{\mathrm{Batt}} \cdot\left(U_{\mathrm{OCV}}-I_{\mathrm{Batt}} \cdot R_{\mathrm{i}}-I_{\mathrm{Batt}} \cdot R_{\mathrm{C}} \cdot\left(1-e^{\frac{-t}{C \cdot R_{C}}}\right)\right)
$$




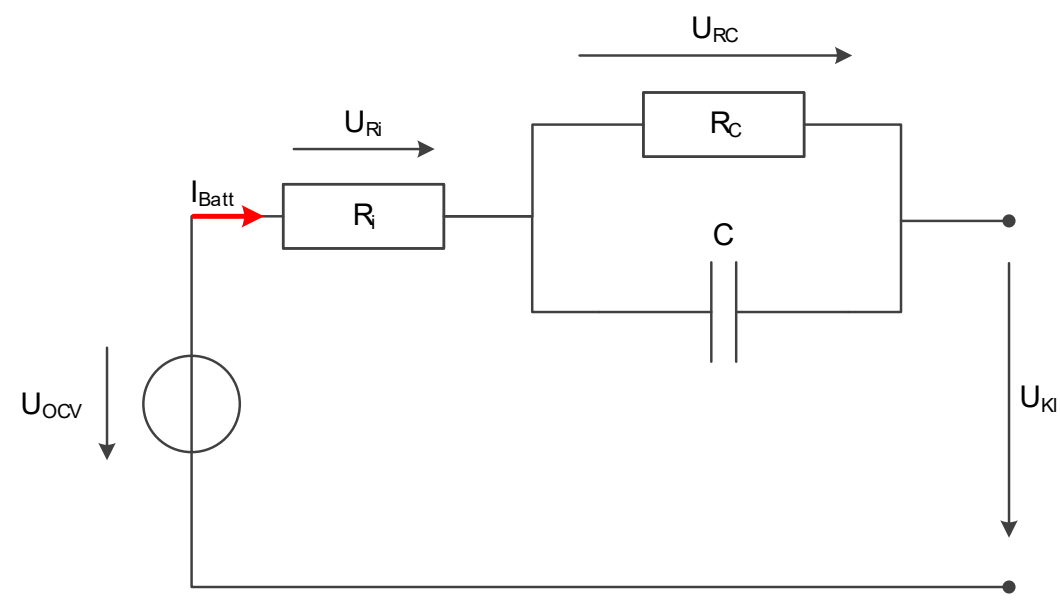

Abbildung 4-4: Elektrisches Zell- und Batteriemodell

Durch Umstellen der Gleichung (4-6) und Lösen des quadratischen Zusammenhangs ergibt sich der Batteriestrom zu:

$$
I_{\text {Batt }}=\frac{U_{\mathrm{OCV}}}{2\left(R_{\mathrm{i}}+R_{\mathrm{C}} \cdot\left(1-e^{\frac{-t}{C \cdot R_{C}}}\right)\right)}-\sqrt{\left(\frac{U_{\mathrm{OCV}}}{2\left(R_{\mathrm{i}}+R_{\mathrm{C}} \cdot\left(1-e^{\frac{-t}{C \cdot R_{C}}}\right)\right)}\right)^{2}-\frac{P_{\mathrm{Batt}}}{2}}
$$

Die Parameter der Ersatzschaltung sind keine konstanten Größen. In Tabelle 4-4 sind die einzelnen Bestandteile des ESB und ihre Abhängigkeiten gegeben.

Tabelle 4-4: Parameter des elektrischen ESB und ihre Abhängigkeiten

\section{OCV und Kapazität Ohmscher Innenwiderstand RC-Glied}

OCV abhängig vom SOC abhängig von Temperatur abhängig von

Kapazität abhängig von und $\mathrm{SOC}$

Temperatur und SOC

Temperatur

\section{OCV und Kapazität}

Die Ruhespannung der Batterie ist eine Funktion des Ladezustands und die dazugehörige Ruhespannungskennlinie oftmals im Datenblatt einer Zelle angegeben. Ist dies nicht der Fall, so kann die Kennlinie messtechnisch ermittelt werden. Dafür existieren zwei bekannte Verfahren, welche u.a. in (Wackernagel, 2012) und (Richter, 2017) beschrieben werden: 
- Voltage Relaxation Method (VRM),

- Linear Interpolation Method (LIM).

Bei Anwendung der VRM wird die Zelle auf verschiedene Ladezustände geladen. Anschließend folgt jeweils eine längere Pause, sodass alle internen Ausgleichsvorgänge abklingen und die tatsächliche Ruhespannung gemessen werden kann. Diese Methode ist aufgrund der langen Messdauer sehr zeitaufwendig. Bei Verwendung der LIM kann die Messzeit deutlich reduziert werden. Dabei wird ein sehr kleiner, konstanter Strom in die Zelle eingeprägt und diese damit je einmal vollständig geladen und wieder entladen. Aufgrund des sehr kleinen Batteriestroms können die resultierenden Überspannungen in guter Näherung vernachlässigt werden. Die gemessene Klemmenspannung entspricht somit ungefähr der Ruhespannung der Zelle. Die resultierende Ruhespannungskennlinie wird nun über die Mittelung der beiden Kurven (laden und entladen) berechnet (Meubrink, 2016; Richter, 2017). In (Richter, 2017) wurde eine Kombination der beiden Verfahren zu Ermittlung der Ruhespannungskennlinie für die $\mathrm{LiFePO}_{4}$ Zelle angewendet. Die ermittelte Kennlinie ist in Abbildung 4-5 gezeigt.

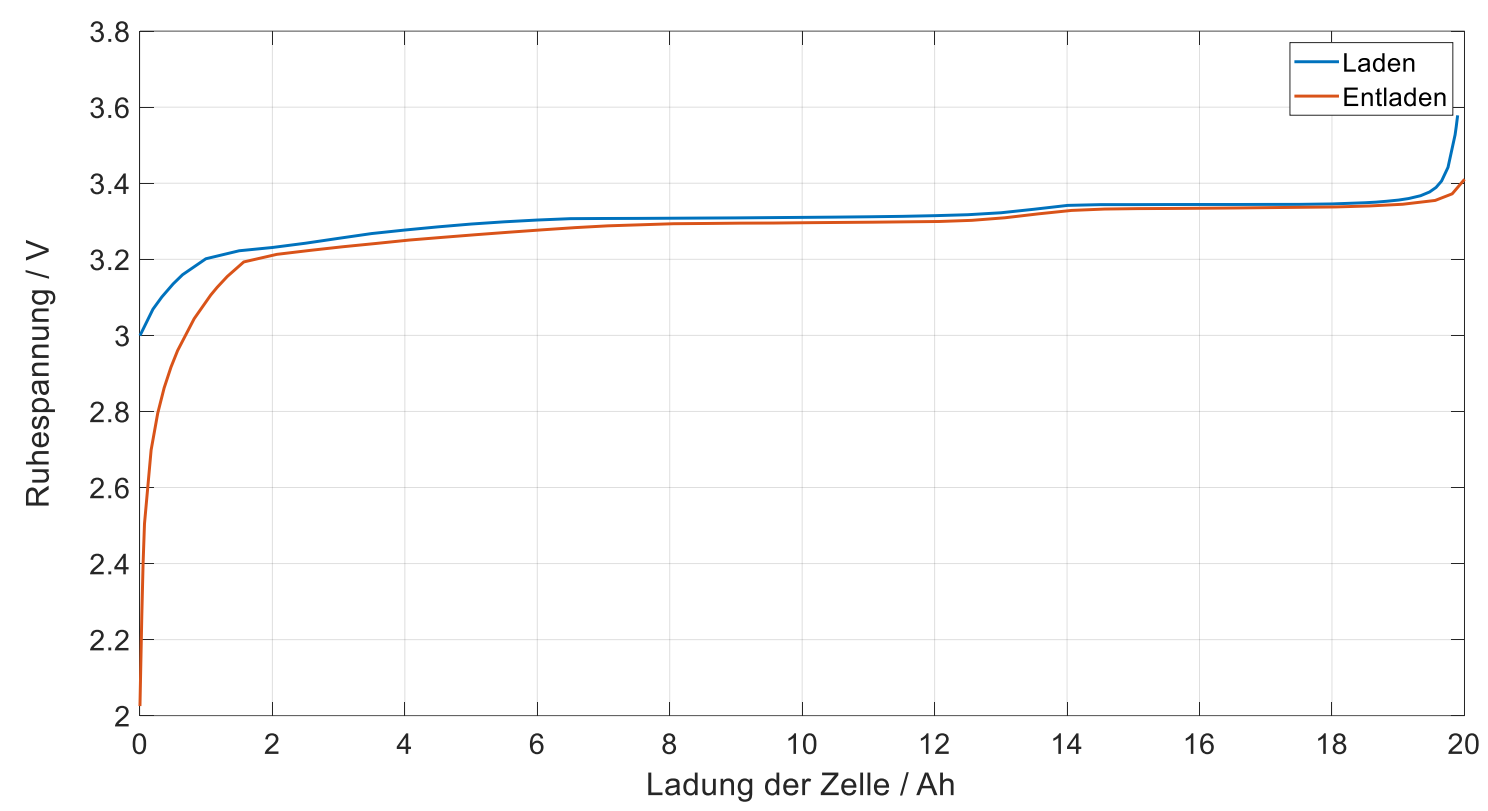

Abbildung 4-5: OCV-Kennlinie der $\mathrm{LiFePO}_{4}$ Zelle (Richter, 2017)

Die Ruhespannungskennlinie ist generell zellabhängig und muss für jede Zelle einzeln ermittelt werden. Auch Zellen mit gleicher Zellchemie (z.B. NMC oder LiFePO 4 ) können unterschiedliche Ruhespannungscharakteristiken aufweisen. Eine generische Ruhespannungskennlinie, welche für verschiedene Zelltypen oder Zellchemien universell verwendet werden kann, existiert nicht. 
Die nutzbare Kapazität einer Lithium-Ionen-Zelle ist abhängig von deren Temperatur. Im Datenblatt ist oftmals die Kapazität der Zelle bei Nennbedingungen (meist bei $25^{\circ} \mathrm{C}$ ) angegeben. Vor allem bei niedrigen Temperaturen kann die tatsächlich verfügbare Kapazität jedoch stark vom Nennwert abweichen. In Abbildung 4-6 sind Entladekurven der $\mathrm{LiFePO}_{4}$ Zelle bei verschiedenen Temperaturen angegeben. Der Entladestrom von 19,5 A entspricht einer Entladerate von 1C. Es ist deutlich erkennbar, dass die verfügbare Kapazität vor allem bei sehr niedrigen Temperaturen stark absinkt. Die Kenntnis über die tatsächliche Batterietemperatur ist demnach essentiell für eine valide Dimensionierung einer Elektrobuslinie. Eine thermische Batteriesimulation ist somit zwingend erforderlich (Vgl. Kapitel 0).

19.5 A Discharge, $45^{\circ} \mathrm{C}$ to $-30^{\circ} \mathrm{C}$

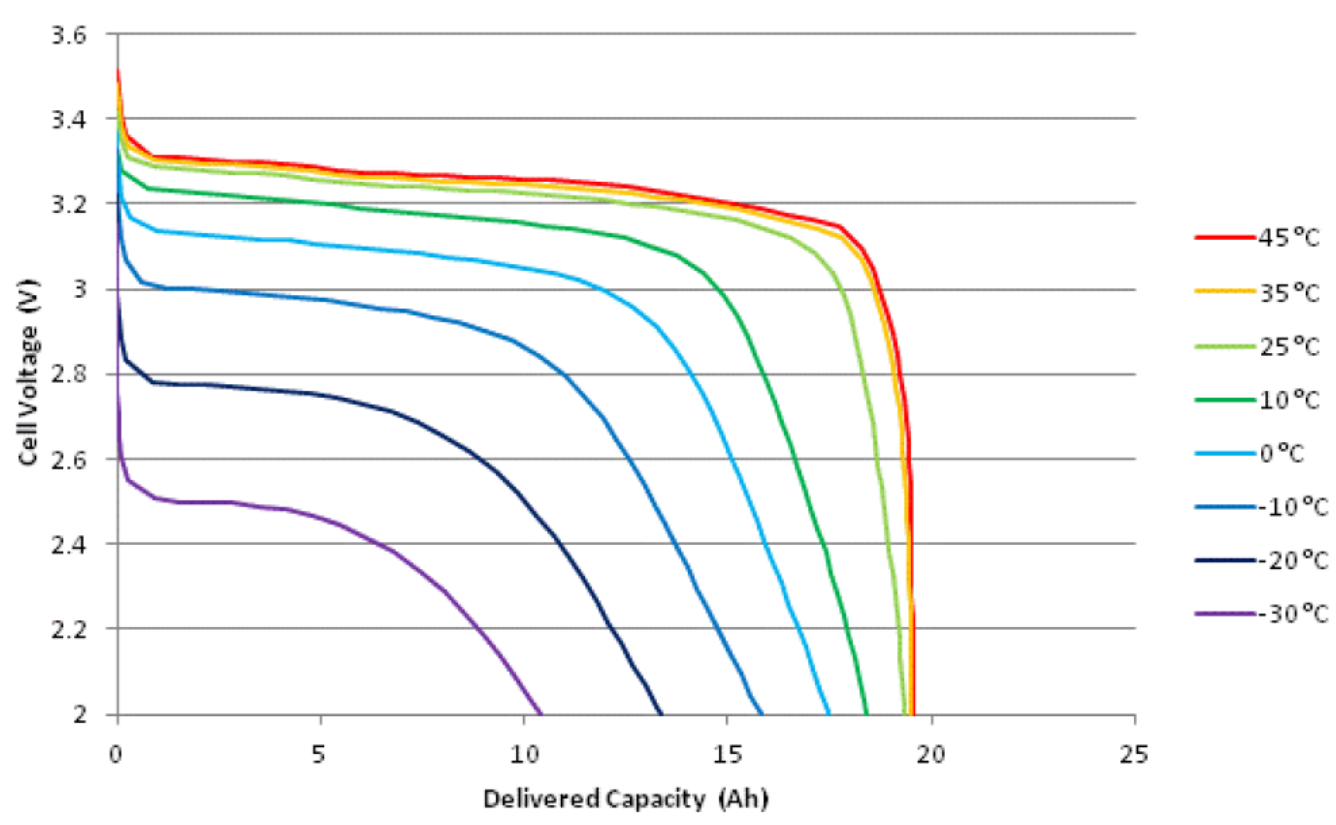

\section{Abbildung 4-6: Temperaturabhängige Entladekurven der $\mathrm{LiFePO}_{4} \mathrm{Zelle}$ (A123 Systems, 2014)}

\section{Ohmscher Innenwiderstand}

In (Meubrink, 2016) wurde anhand verschiedener Messungen an der $\mathrm{LiFePO}_{4}$ Zelle gezeigt, dass der ohmsche Innenwiderstand sowohl eine Temperaturabhängigkeit als auch eine SOC-Abhängigkeit aufweist. Aus den Messergebnissen geht hervor, dass die SOC-Abhängigkeit gegenüber der Temperaturabhängigkeit sehr gering ausgeprägt ist. Das Kennfeld des Innenwiderstands ist in Abbildung 4-7 gegeben. Es ist erkennbar, dass $\mathrm{R}_{\mathrm{i}}$ oberhalb von $25^{\circ} \mathrm{C}$ nahezu konstant ist. Unterhalb von $25^{\circ} \mathrm{C}$ stellt sich jedoch ein nichtlineares Verhalten ein, wobei die Temperaturabhängigkeit dabei deutlicher ausgeprägt ist als die SOC-Abhängigkeit. Als Grund für den überproportional großen 
Wert im Bereich von $-10{ }^{\circ} \mathrm{C}$ und ca. $40 \%$ SOC wird eine Messungenauigkeit vermutet. In (Meubrink, 2016) werden dazu jedoch keine Angaben gemacht.

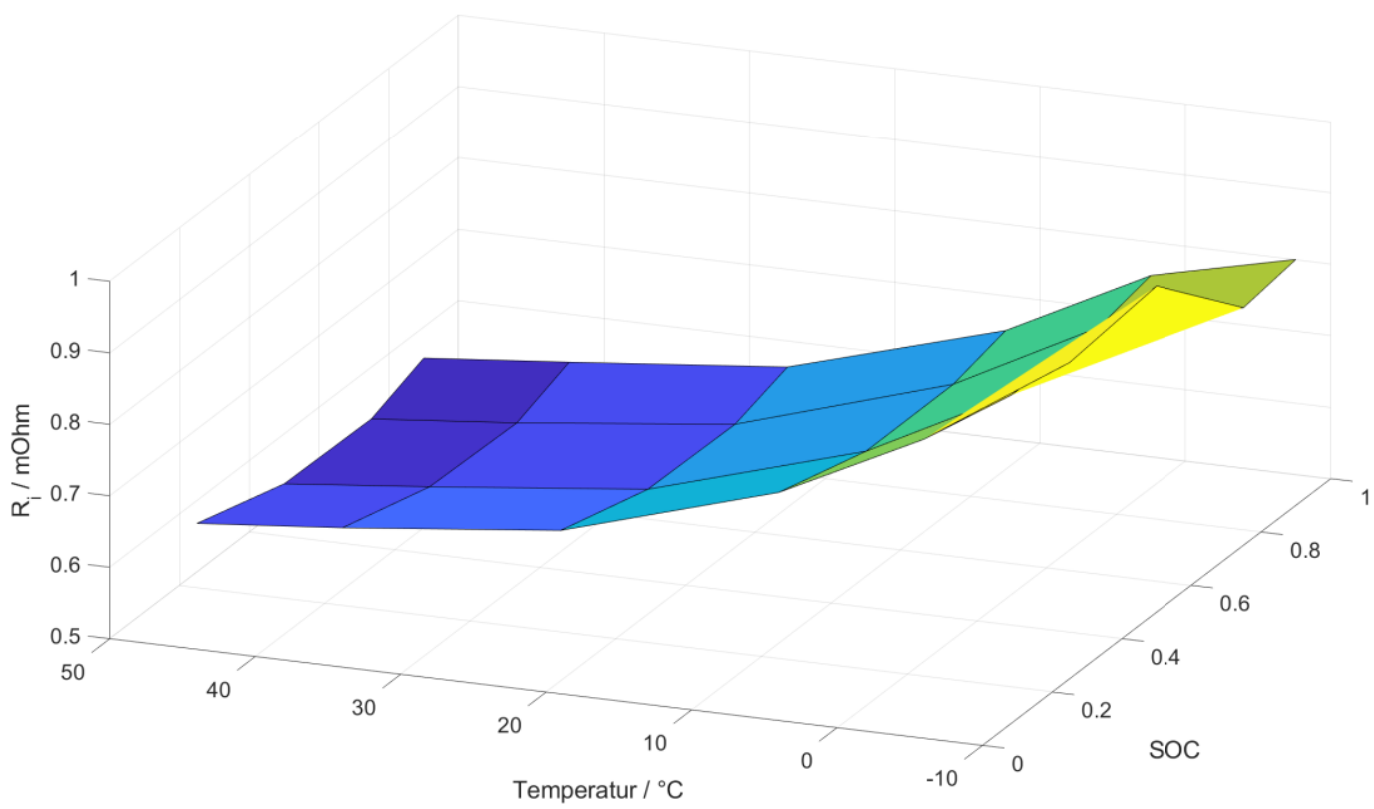

\section{Abbildung 4-7: Kennfeld ohmscher Innenwiderstand $\mathbf{R}_{\mathbf{i}}$, Messdaten aus (Meubrink, 2016)}

\section{RC-Glied}

Die Parameter des RC-Glieds sind oftmals nicht in Datenblättern angegeben. Eine Ermittlung ist über das bekannte Verfahren der elektrochemischen Impedanzspektroskopie (EIS) möglich. Das Verfahren ist unter anderem in (Richter, 2017) detailliert beschrieben, weshalb an dieser Stelle nur die für diese Arbeit erforderlichen Ergebnisse der Messung dargestellt werden. Die gemessene Ortskurve der $\mathrm{LiFePO}_{4}-$ Zelle ist in Anhang E.1 gegeben. Dafür wurde das Messsystem Reference 3000 des Herstellers Gamry verwendet. Über die darin enthaltene Software zum Postprocessing Gamry Echem Analyst können mittels einer Fitting-Funktion die Parameter der Ortskurve bestimmt werden:

$$
\begin{aligned}
R_{\mathrm{i}} & =0,786 \mathrm{~m} \Omega \\
R_{\mathrm{C}} & =1,537 \mathrm{~m} \Omega \\
C & =3,351 \mathrm{~F}
\end{aligned}
$$


Die Parametrierung wurde bei einer Temperatur von $25^{\circ} \mathrm{C}$ und einem Ladezustand von $50 \%$ durchgeführt. Der Wert des ermittelten ohmschen Innenwiderstands $R_{\mathrm{i}}$ stimmt mit dem aus den Messungen in (Meubrink, 2016) überein (Vgl. Abbildung 4-7). In (Richter, 2017) wurde eine Temperatur- und SOC-Abhängigkeit des RC-Glieds messtechnisch ermittelt. Im weiteren Verlauf dieser Arbeit wird jedoch mittels einer Validierung gezeigt, dass auch ohne diese Abhängigkeit eine sehr gute Modellgüte erreicht wird, weshalb im Rahmen dieser Arbeit auf eine temperatur- und SOCabhängige Parametrierung des RC-Glieds verzichtet wird.

\section{Betriebsgrenzen}

Zum sicheren und zuverlässigen Betrieb einer Traktionsbatterie werden vom Zellhersteller bzw. vom Batteriehersteller die zulässigen Betriebsbereiche eingeschränkt. Dies dient zum einen der Sicherheit, zum anderen aber auch der Langlebigkeit des Systems. In der elektrisch-thermischen Simulation werden zunächst die Betriebsgrenzen aus Sicht der Systemsicherheit definiert. Eine weitere Einschränkung des Betriebsbereichs unter Langlebigkeitsaspekten wird in Kapitel 4.1.5 behandelt.

Zur Gewährleistung der Sicherheit einer Li-lonen Zelle müssen im Wesentlichen zwei Größen beachtet werden. Dies ist zum einen die Batterie- bzw. Zelltemperatur. Für jede Batterie ist eine maximale Temperatur definiert, welche aus Sicherheitsgründen nicht überschritten werden darf. Bei tiefen Temperaturen besteht zudem eine Sicherheitsgefahr durch Lithium-Plating ${ }^{12}$ (Fischhaber et al., 2016). Zudem muss die Spannung der Zellen innerhalb der zulässigen Grenzen gehalten werden, um eine Überladung bzw. eine Tiefentladung zu verhindern. Die Spannungsgrenzen sind für verschiedene Zellchemien unterschiedlich (Vgl. Abbildung 4-2). Die Spannungsgrenzen sind sowohl beim Laden als auch beim Entladen zu beachten. Beim Entladen muss sichergestellt werden, dass den Zellen nur so lange eine Ladung entnommen wird, wie die Zellspannung über der Entladeschlussspannung liegt. Bis zum Erreichen dieser kann die Zelle mit dem max. zulässigen Entladestrom belastet werden. Der Zusammenhang beim Laden stellt sich jedoch anders dar. Zur Vermeidung eines Überschreitens der Ladeschlussspannung wird üblicherweise ein zweistufiges Ladeverfahren verwendet, das s.g. Constant-Current-Constant-Voltage-Verfahren (CCCV). Das Prinzip ist in Abbildung 4-8 dargestellt und in (Jossen et al., 2006) detailliert erläutert. In der CCPhase wird die Zelle mit einem konstanten Strom geladen. Dabei steigen sowohl die Ruhespannung (und damit der SOC) als auch die Klemmenspannung kontinuierlich an. Erreicht die Klemmenspannung die Ladeschlussspannung, muss der Ladestrom begrenzt werden, um die Spannung nicht über den maximalen Wert ansteigen zu

\footnotetext{
${ }^{12}$ Als Lithium-Plating wird die Ablagerung von metallischem Lithium an der Grenze zwischen der SEI und dem Aktivmaterial der Anode bezeichnet. Dabei bilden sich Dendriten aus, welche den Separator beschädigen und zu einem inneren Kurzschluss der Zelle führen können (Fischhaber et al., 2016).
} 
lassen, wodurch die jeweilige Zelle nachhaltig beschädigt werden würde. Es schließt sich die CV-Phase an. Darin wird die Ladespannung konstant gehalten, wodurch der Ladestrom entsprechend absinkt. Erreicht der Ladestrom einen unteren Schwellwert (oft bei C/20), wird die Ladung beendet.

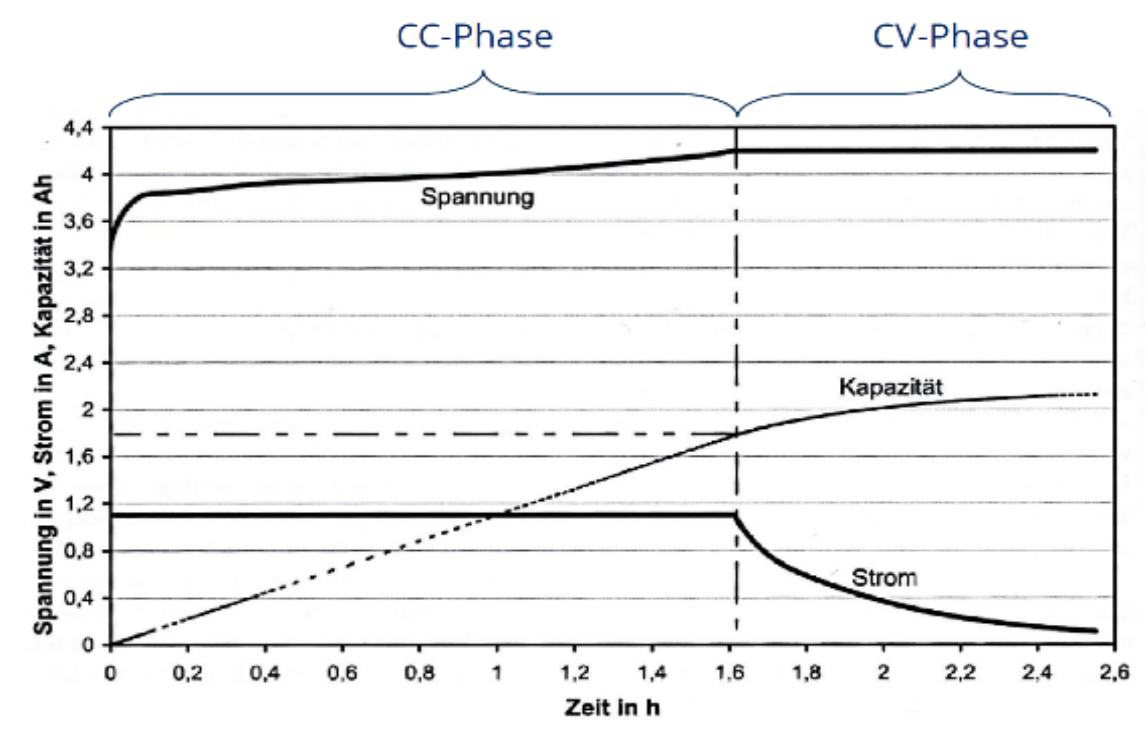

Abbildung 4-8: Beispiel einer Ladung im CC-CV-Verfahren (Jossen et al., 2006)

Zur Umsetzung des CCCV-Ladeverfahrens wird ein Stromregler benötigt. Dessen Funktion kann über die Implementierung eines Kennfelds nachgebildet werden. Dafür wird der Zusammenhang zwischen Ladezustand (in Abbildung 4-8 als Kapazität bezeichnet) und Strom genutzt. Durch das exponentielle Absenken des Ladestroms bei Erreichen eines Schwellwertes des Ladezustands kann eine Verletzung der oberen Spannungsgrenze ausgeschlossen werden. Des Weiteren muss die Temperatur als wichtige Betriebsgrenze einer Li-Ionen Batterie beachtet werden. Ein Übersteigen der maximal zulässigen Temperatur kann zu einem thermischen Durchgehen der Zelle (thermal runaway) führen und diese damit nachhaltig beschädigen. Bei konstanter Umgebungstemperatur ist die Zelltemperatur hauptsächlich vom Batteriestrom abhängig. In den ohmschen Widerständen der Batterie wird gemäß Formel (4-8) ein Wärmestrom eingeprägt und die Zelle somit erwärmt. Bei Erreichen eines Temperaturschwellwertes muss der Batteriestrom demnach heruntergeregelt werden, um eine Überhitzung der Zelle zu vermeiden. Dies geschieht im Sinne eines effizienten Betriebs keinesfalls sprungartig. Vielmehr wird der Strom entlang mehrerer Stützstellen kontinuierlich an die jeweilige Betriebssituation angepasst. Der oben beschriebene Stromregler muss neben dem Ladezustand somit auch die Batterietemperatur berücksichtigen.

$$
P_{\text {Verlust }}=I_{\text {Batt }}^{2} \cdot R
$$


Beide erläuterten Zusammenhänge, die Abhängigkeit des zulässigen Batteriestroms vom Ladezustand und der Temperatur, werden von Herstellern oftmals im erweiterten Datenblatt als dreidimensionales Kennfeld gegeben, beispielsweise in (A123 Systems, 2014). Darin wird zwischen den Betriebsfällen Ladung und Entladung unterschieden.
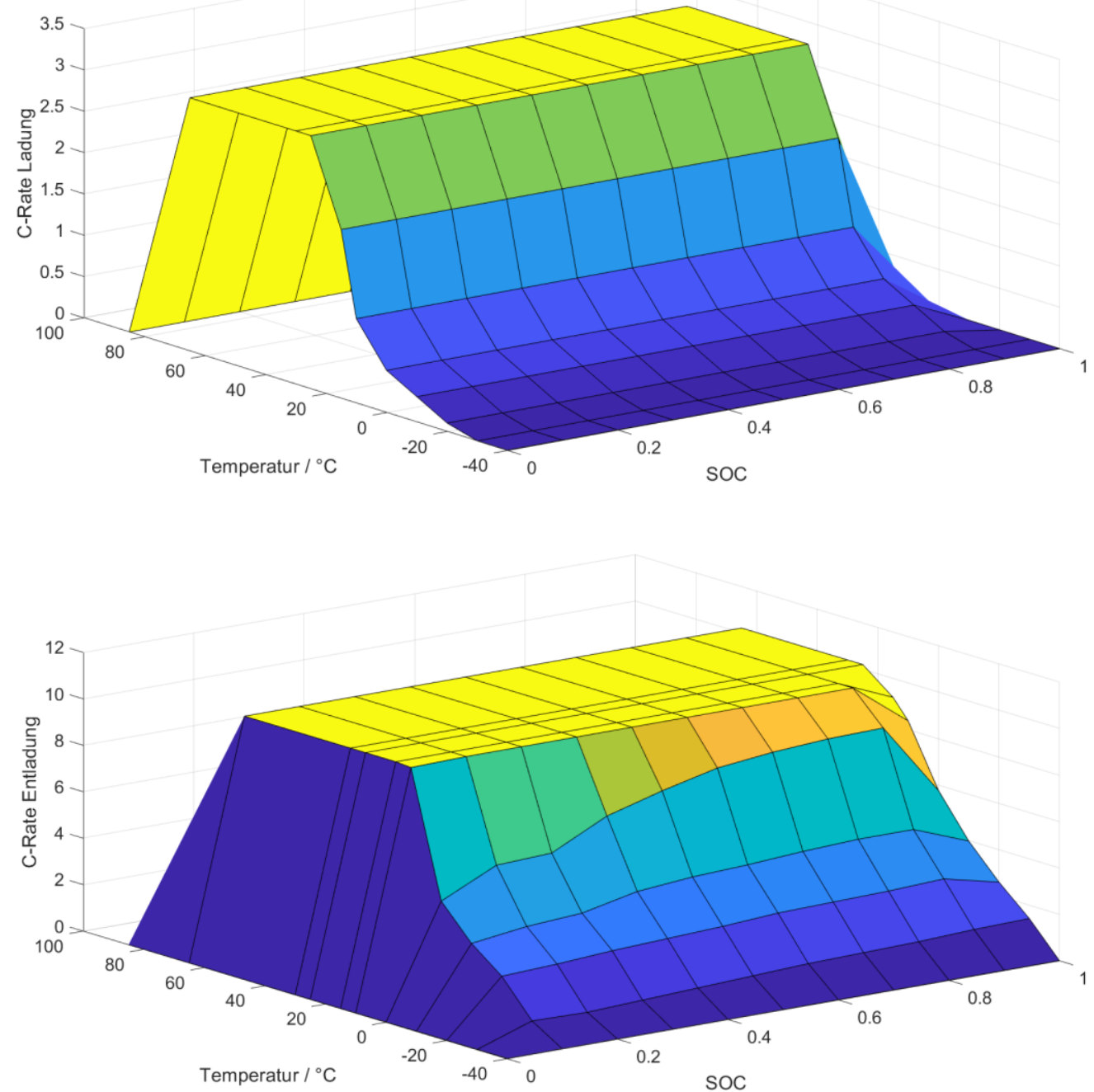

Abbildung 4-9: Kennfelder Strombegrenzung der $\mathrm{LiFePO}_{4} \mathrm{Zelle}$ (oben: Laden, unten: Entladen), Daten aus (A123 Systems, 2014)

Das Kennfeld der in dieser Arbeit verwendeten $\mathrm{LiFePO}_{4}$ Zelle von A123 ist in Abbildung 4-9 gegeben. Aus der Abbildung geht hervor, dass:

- der Ladestrom im Bereich hoher Ladezustände begrenzt wird (wie nach Abbildung 4-8 und CCCV-Ladeverfahren gefordert), 
- die Zelle bis nahezu 0\% Ladezustand mit vollem Entladestrom belastet werden kann,

- sowohl der Ladestrom als auch der Entladestrom nicht nur bei hohen, sondern auch bei niedrigen Temperaturen begrenzt wird (Grund: Vermeidung von Lithium-Plating (Fischhaber et al., 2016)).

Zusätzlich können aus Abbildung 4-9 zwei weitere Aspekte zum Betrieb der Zelle abgeleitet werden:

- Entladeströme werden deutlich weniger begrenzt als Ladeströme. Der maximale Wert liegt mit ca. 10C beim Entladen um mehr als Faktor 3 höher als beim Laden (ca. 3C). Dies kann mit der unterschiedlichen Volumenarbeit der Elektroden begründet werden. Während Anoden auf Graphitbasis eine Volumenarbeit von 9,3\% aufweisen, haben $\mathrm{LiFePO}_{4}$ Kathoden nur 6,8\% (Jossen et al., 2019).

- Die Zelle entfaltet ihre maximale Leistungsfähigkeit über einen sehr weiten Temperaturbereich (ca. $25^{\circ} \mathrm{C}$ bis $65^{\circ} \mathrm{C}$ ), da LiFePO 4 Zellen generell eine hohe Sicherheit aufweisen (Vgl. Abbildung 4-2). Die konkreten Werte sind jedoch abhängig von zellspezifischen Eigenschaften, sodass aus den Kennfeldern kein allgemeingültiger Zusammenhang abgeleitet werden kann.

\section{Validierung}

Gemäß Tabelle 4-1 wird das elektrische Modell auf Zellebene validiert. Dafür werden im Folgenden eine Messung und eine Simulation miteinander verglichen. Eine Einzelzelle wurde unter Laborbedingungen bei einer nahezu konstanten Umgebungstemperatur von $50^{\circ} \mathrm{C}$ mit einem vorgegebenen Stromprofil belastet, welches einem möglichen Lastzyklus eines Stadtbusses entspricht. Für die Messung wurden das Batterietestsystem XTCS der Firma BaSyTec und die Klimakammer VT 4021 EMC der Firma Vötsch verwendet. Die verwendete Messtechnik ist in (Richter, 2017) ausführlich beschrieben.

Das entwickelte elektrische Modell ist jedoch nicht strom-, sondern leistungsgeführt (Vgl. Formel (4-7)). Deshalb wird für die Validierung das elektrische Modell mit einer Leistung beaufschlagt, welche aus dem eingeprägten Stromprofil und der gemessenen Klemmenspannung berechnet wurde. In Abbildung 4-10 sind der ermittelte Leistungsverlauf (oben) sowie der resultierende SOC-Verlauf (unten) dargestellt. Unter Anwendung der Formel (4-7) wird nun der Laststrom der Zelle simuliert. Die Ergebnisse der Messung und der Simulation des Stroms sind in Abbildung 4-11 (oben) vergleichend dargestellt. Es ist erkennbar, dass die absolute Abweichung, welche aus der Differenz aus Messung und Simulation resultiert und in Abbildung 4-11 (unten) dargestellt ist, 
im Bereich von kleiner als +900 mA bzw. -400 mA liegt. Der Effektivwert des gemessenen Stroms beträgt 10,9 A und der Effektivwert der berechneten Differenz $52 \mathrm{~mA}$. Aus dem Verhältnis der beiden Werte ergibt sich ein relativer Modellfehler von rund 0,5\%. In Abbildung 4-12 ist eine Ausschnitt aus Abbildung 4-11 dargestellt. Es ist erkennbar, dass beide Signale nahezu deckungsgleich übereinander liegen.
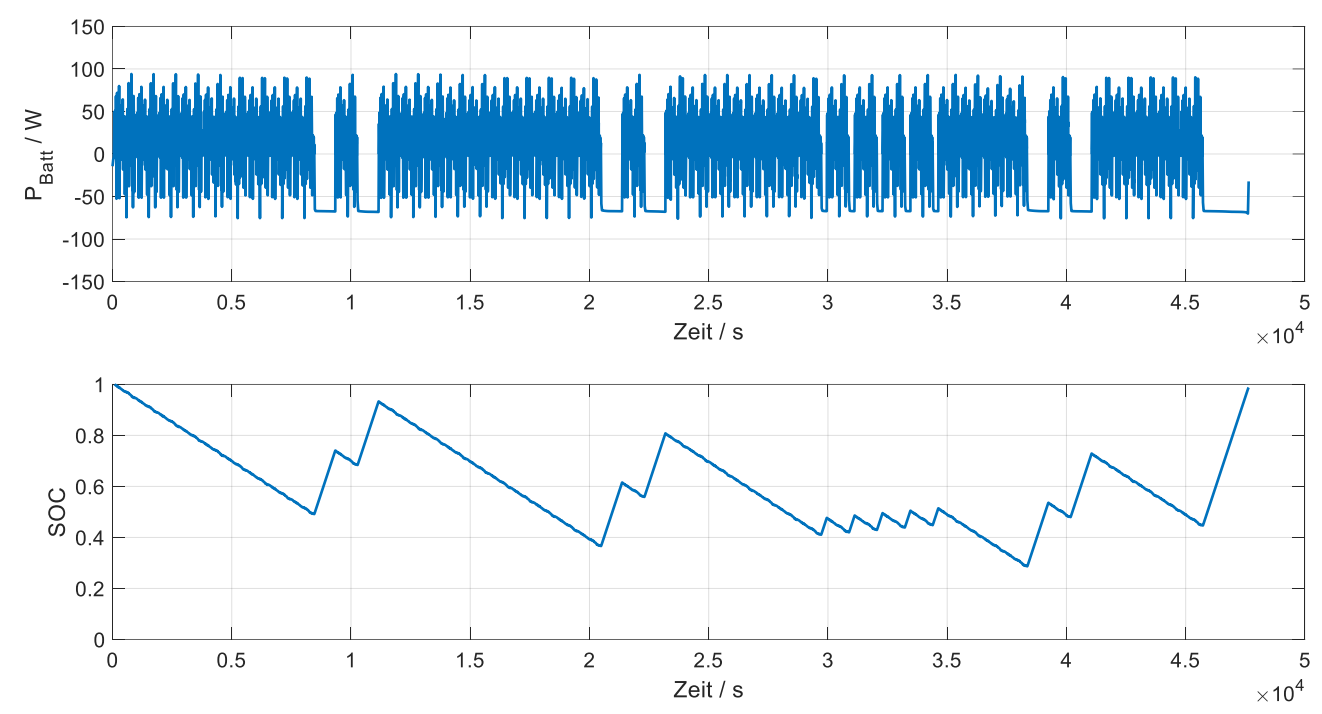

\section{Abbildung 4-10: Gemessener Leistungs- und SOC-Verlauf für die Validierung des elektrischen Modells}
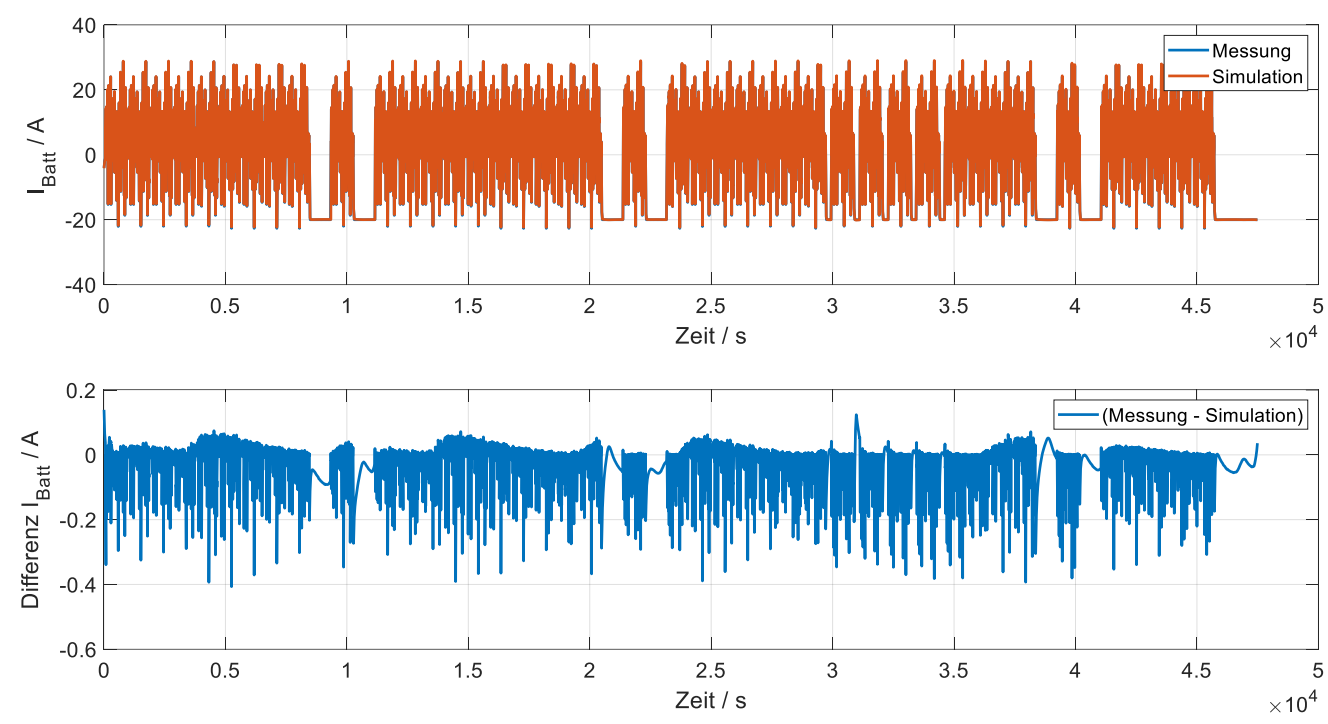

Abbildung 4-11: Vergleich Simulation und Messung - Batteriestrom $I_{\text {Batt }}$

In Abbildung 4-13 (oben) sind die Ergebnisse der Messung und der Simulation der Klemmenspannung vergleichend dargestellt. Die Abweichung ist in Abbildung 4-13 
(unten) gezeigt. Es ist erkennbar, dass die Differenz aus Messung und Simulation im Bereich von $\pm 50 \mathrm{mV}$ liegt. Der Effektivwert der gemessenen Klemmenspannung beträgt 3,3 V und der Effektivwert der Differenz beträgt $17 \mathrm{mV}$. Somit ergibt sich auch hier ein relativer Modellfehler von 0,5\%. In Abbildung 4-14 ist ein Ausschnitt aus Abbildung 4-13 abgebildet. Es ist erkennbar, dass die Dynamik der Simulation in gutem Maße mit der des Messsignals übereinstimmt. Die Validierungsergebnisse des elektrischen Modells sind in Tabelle 4-5 zusammengefasst.
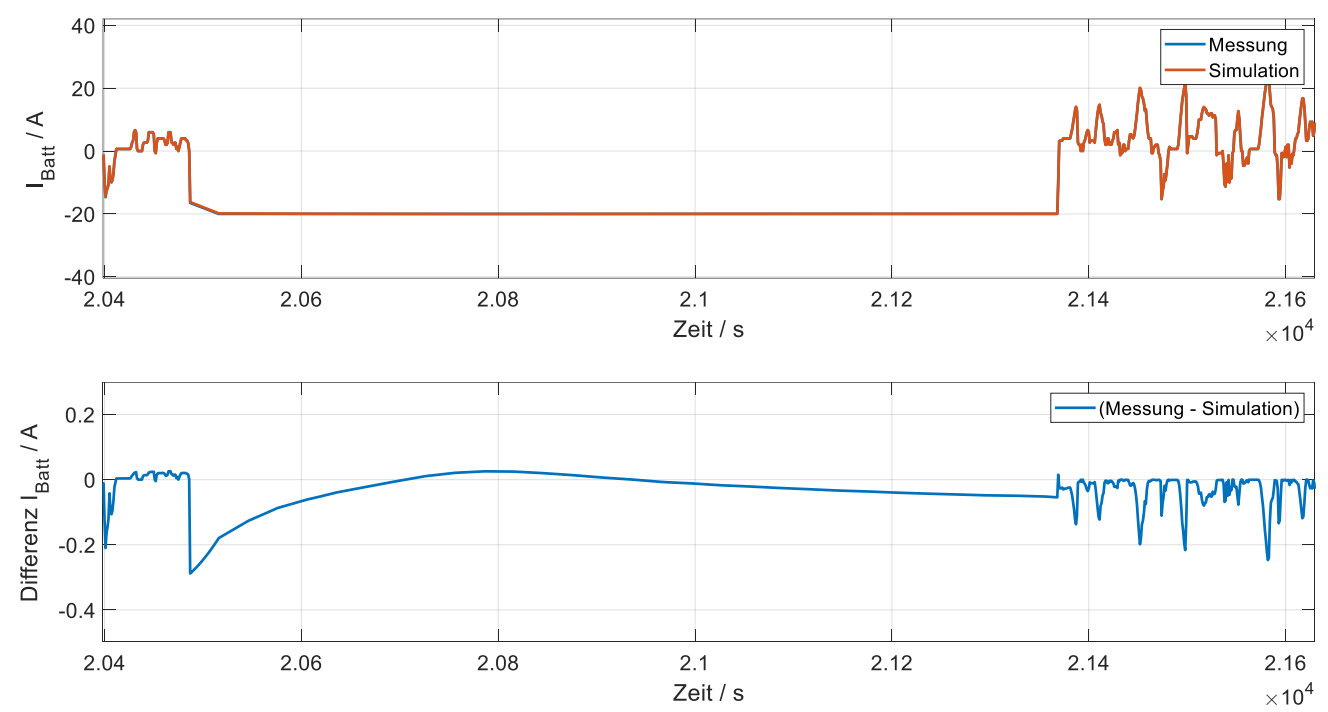

Abbildung 4-12: Vergleich Simulation und Messung - Batteriestrom I Batt $_{\text {(Ausschnitt) }}$
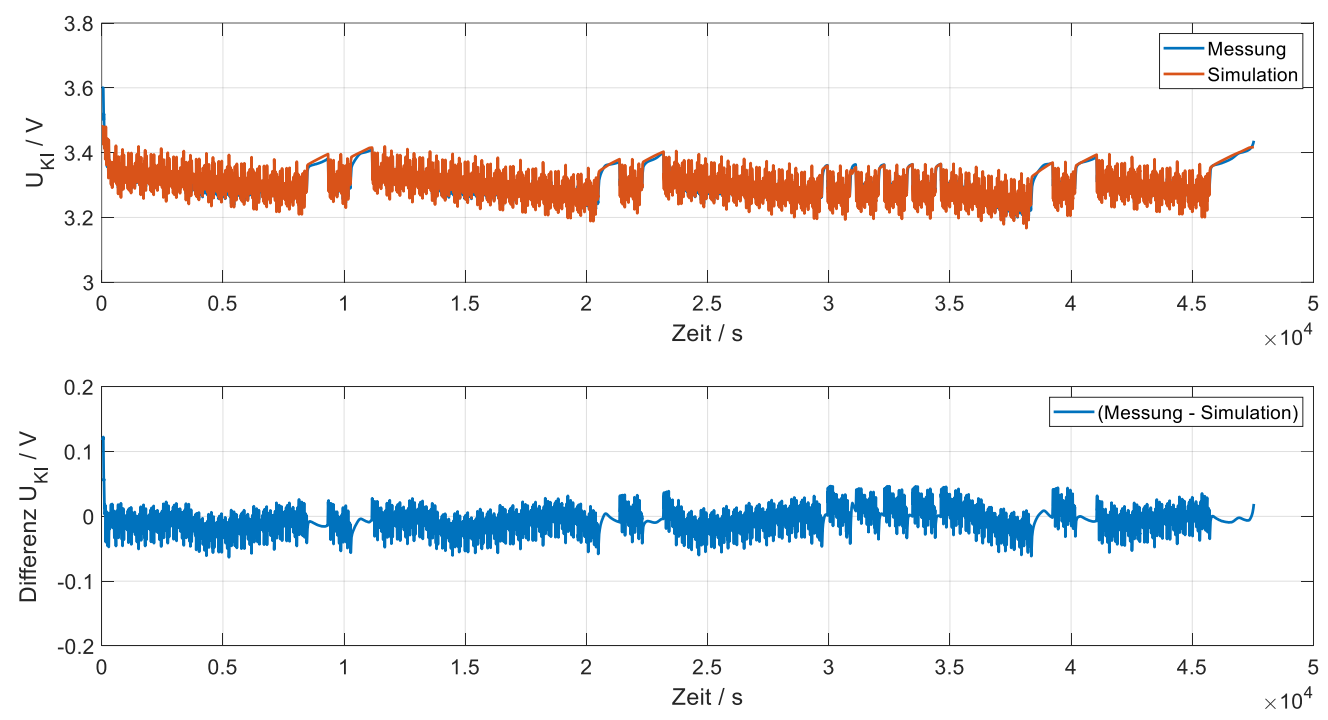

Abbildung 4-13: Vergleich Simulation und Messung - Klemmenspannung $U_{\mathrm{KI}}$ 

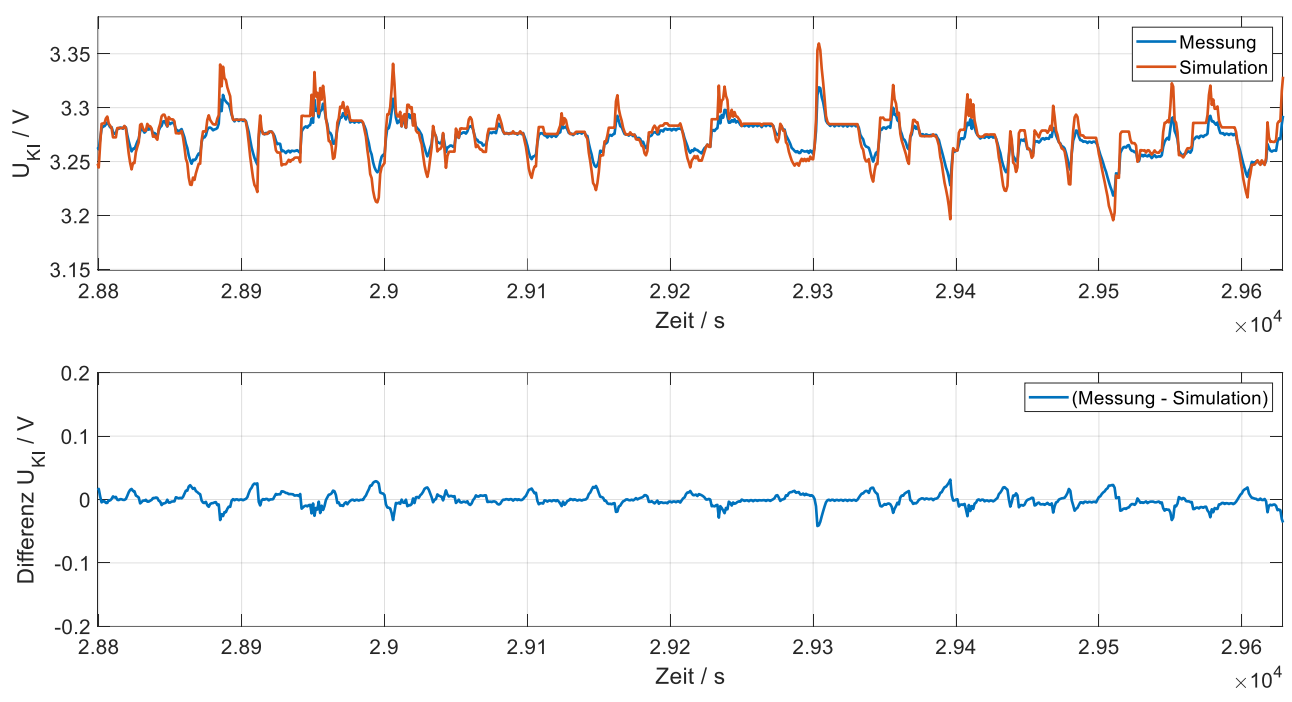

Abbildung 4-14: Vergleich Simulation und Messung - Klemmenspannung $U_{K I}$ (Ausschnitt)

Tabelle 4-5: Zusammenfassung der Validierungsergebnisse der elektrischen Modellbildung

\begin{tabular}{lcc}
\hline & Stromverlauf & Spannungsverlauf \\
\hline max. Abweichung & $+0,9 \mathrm{~A}$ & $+0,23 \mathrm{~V}$ \\
(absoluter Fehler) & $-0,4 \mathrm{~A}$ & $-0,06 \mathrm{~V}$ \\
RMS-Wert Abweichung & $0,052 \mathrm{~A}$ & $0,017 \mathrm{~V}$ \\
RMS-Wert gemessenes & $10,9 \mathrm{~A}$ & $3,3 \mathrm{~V}$ \\
Profil & $0,5 \%$ & $0,5 \%$ \\
relativer Fehler & & 0 \\
\hline
\end{tabular}

\subsubsection{Thermisches Modell}

In diesem Abschnitt wird ein Modell zur Beschreibung des thermischen Verhaltens einer einzelnen Li-lonen Zelle und eines Batteriemoduls entwickelt. Dafür werden im Folgenden zunächst einige thermische Kenngrößen eingeführt sowie notwendige Grundlagen zur thermischen Modellbildung gelegt.

In (Richter, 2017) werden unterschiedliche Vorgänge zur Wärmeentstehung in Li-Ionen Zellen genannt. Der Wärmestrom $\dot{Q}$ setzt sich hauptsächlich aus den folgenden vier Bestandteilen zusammen. 


$$
\begin{array}{ll} 
& \dot{Q}=\dot{Q}_{\text {irr }}+\dot{Q}_{\text {rev }}+\dot{Q}_{\text {reakt }}+\dot{Q}_{\text {mix }} \\
\dot{Q}_{\text {irr }} \quad \text { irreversible Wärmestrom } \\
\dot{Q}_{\text {rev }} \quad \text { reversibler Wärmestrom } \\
\dot{Q}_{\text {reakt }} \quad \text { Wärmestrom durch Nebenreaktionen } \\
\dot{Q}_{\text {mix }} \quad \text { Wärmestrom durch Mischungsenthalpie }
\end{array}
$$

Nach (Richter, 2017) können die beiden letztgenannten Anteile $\left(\dot{Q}_{\text {reakt }}+\dot{Q}_{\text {mix }}\right)$ in guter Näherung vernachlässigt werden. Der reversible Wärmestrom $\dot{Q}_{\text {rev }}$ wurde in (Richter, 2017) detailliert untersucht. Der Autor kommt zum Ergebnis, dass dieser nur in einem engen SOC-Fenster bei sehr kleinen und sehr großen Ladezuständen zum Tragen kommt. Aus den Untersuchungen geht zudem hervor, dass der Anteil am Gesamtwärmestrom verhältnismäßig klein ist. In dieser Arbeit wird deshalb nur der irreversible Wärmestrom $\dot{Q}_{\text {irr }}$ betrachtet. Es gilt:

$$
\dot{Q} \approx \dot{Q}_{\text {irr }}
$$

Der irreversible Wärmestrom berechnet sich analog zur elektrischen Verlustleistung.

$$
\dot{Q}_{\text {irr }}=R_{\mathrm{i}} \cdot\left(I_{\text {Batt }}\right)^{2}
$$

Neben der Wärmeentstehung ist der Wärmetransport bei der thermischen Modellbildung zu berücksichtigen. Weisen zwei Körper unterschiedliche Temperaturen auf, so findet zwischen ihnen ein Wärmeaustausch statt.

Für den Wärmetransport werden drei Arten unterschieden:

- Wärmeleitung,

- Wärmekonvektion,

- Wärmestrahlung.

In (Kritzinger, 2019) ist eine Übersicht der genannten Wärmetransportarten gegeben, welche in Abbildung 4-15 dargestellt sind. Im Folgenden werden diese detailliert erläutert und wichtige Begriffe und Definitionen zur thermischen Modellbildung eingeführt. 
a)

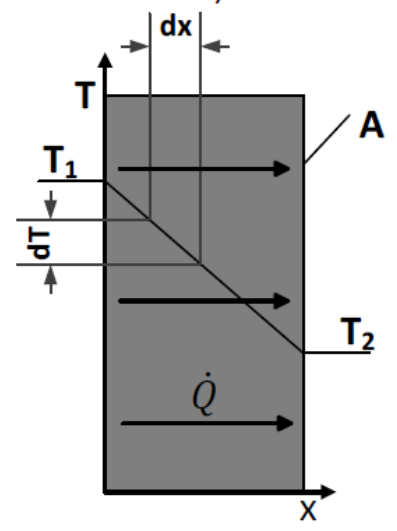

b)

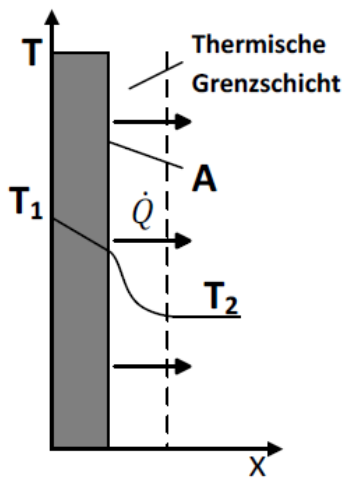

c)

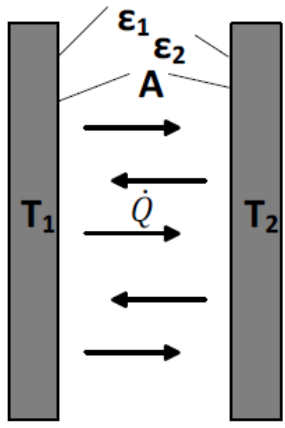

Abbildung 4-15: Wärmeübertragungsprozesse:

a) Wärmeleitung, b) Wärmekonvektion, c) Wärmestrahlung (Kritzinger, 2019)

\section{Wärmeleitung}

In einem festen Körper oder einem ruhenden Fluid wird der Wärmestrom als Wärmeleitung bezeichnet. Im Falle einer Batterie ist die Wärmeleitung dafür verantwortlich, dass Wärme aus dem Inneren der Zelle an die Zellhülle abgegeben wird (Kritzinger, 2018a). Bei Betrachtung zweier paralleler Flächen wird der resultierende Wärmestrom $\dot{Q}_{\text {leit }}$ wie folgt beschrieben (Doering et al., 2016):

$$
\dot{Q}_{\text {leit }}=-\lambda_{\text {th }} \cdot A \frac{d T}{d x}
$$

Darin sind $A$ die Fläche des Zellmaterials und $\lambda_{\text {th }}$ die Wärmeleitfähigkeit. In x-Richtung sinkt die Temperatur mit steigender Entfernung von der Wärmequelle, was in Abbildung 4-15 a) verdeutlicht ist.

\section{Wärmekonvektion}

Wärmekonvektion findet zwischen einem festen Körper und einem Fluid statt (Abbildung 4-15 b)). Der resultierende Wärmestrom $\dot{Q}_{\text {konv }}$ berechnet sich wie folgt (Doering et al., 2016):

$$
\dot{Q}_{\mathrm{konv}}=\alpha_{\mathrm{k}} \cdot A \cdot \Delta T
$$

Darin sind $A$ die Oberfläche des Körpers und $\Delta T$ die Temperaturdifferenz zwischen Körper und Fluid. Der Wärmeübergangskoeffizient $\alpha_{\mathrm{k}}$ ist abhängig von verschiedenen Einflussfaktoren und wird für spezifische Anwendungen oftmals empirisch ermittelt. Er ist abhängig von oberflächen- und fluidspezifischen Kenngrößen sowie von der Art der 
Konvektion (freie oder erzwungene Konvektion). In Batterieanwendungen ist Wärmekonvektion vor allem für den Übergang der Wärme von der Zellaußenhülle an die Umgebung, z.B. an ein Kühlmittel, von Bedeutung. Im inneren der Zelle kann Wärmekonvektion mit guter Näherung vernachlässigt werden (Kritzinger, 2019).

\section{Wärmestrahlung}

Wärmestrahlung findet zwischen zwei Körpern statt, wobei im Gegensatz zu den bisher beschriebenen Wärmeleitprozessen kein Übertragungsmedium benötigt wird (Abbildung 4-15 c)). Jeder Körper mit einer Temperatur oberhalb des absoluten Nullpunktes emittiert Wärme in Form elektromagnetischer Wellen. Außerdem absorbieren Körper Wärme über ihre Oberflächen. Mit Hilfe der stoffspezifischen Emissionsgrade $\varepsilon$ und der Stefan-Boltzmann-Konstante $\sigma$ ergibt sich der resultierende Wärmestrom wie folgt (Doering et al., 2016):

$$
\begin{gathered}
\dot{Q}_{\text {strahl }}=c_{12} \cdot A \cdot\left(T_{1}^{4}-T_{2}^{4}\right) \\
c_{12}=\frac{\sigma}{\frac{1}{\varepsilon_{1}}+\frac{1}{\varepsilon_{2}}-1}
\end{gathered}
$$

Bei Batterieanwendungen findet Wärmestrahlung vor allem an der Außenhülle der Zellen statt. Im Vergleich zur Wärmeleitung und Wärmekonvektion hat die Wärmestrahlung dabei jedoch einen vergleichsweise geringen Einfluss auf die Temperaturentwicklung der Zelle (Kritzinger, 2019).

\section{Wärmekapazität}

Neben den Wärmetransportprozessen hat die Wärmekapazität eines Batteriesystems entscheidenden Einfluss auf dessen thermisches Verhalten. Analog zur elektrischen Kapazität wirkt der Körper als Speicher, welcher Wärme aufnehmen und auch wieder abgeben kann. Die Wärmekapazität $C_{\text {th }}$ eines Körpers beschreibt die Temperaturänderung in Abhängigkeit der zugeführten Wärme (Doering et al., 2016):

$$
C_{\mathrm{th}}=\frac{d Q}{d T}
$$

Die Wärmekapazität $C_{\text {th }}$ einer Batteriezelle kann über die stoffspezifische Kapazität $c_{\mathrm{p}}$ und ihre Masse $m_{\text {Zelle }}$ bestimmt werden.

$$
C_{\text {th }}=m_{\text {Zelle }} \cdot c_{\mathrm{p}}
$$




\section{Thermischer Widerstand}

Der thermische Widerstand bzw. Wärmewiderstand beschreibt die Durchlässigkeit eines Materials bzw. eines Körpers und berechnet sich wie folgt:

$$
R_{\mathrm{th}}=\frac{\Delta T}{\dot{Q}}
$$

Es gelten analog zum ohmschen Gesetz beim elektrischen Widerstand die Regeln der Reihen- und Parallelschaltung. Entsprechend der oben beschriebenen Wärmetransportprozesse wird zwischen den thermischen Widerständen für Wärmeleitung, Wärmekonvektion und Wärmestrahlung unterschieden (Doering et al., 2016):

$$
\begin{aligned}
& R_{\mathrm{th}, \text { leit }}=\frac{l}{\lambda_{\mathrm{th}} \cdot A} \\
& R_{\mathrm{th}, \mathrm{konv}}=\frac{1}{\alpha_{\mathrm{k}} \cdot A} \\
& R_{\mathrm{th}, \mathrm{strahl}}=\frac{1}{\alpha_{\varepsilon} \cdot A} \\
& \alpha_{\varepsilon}=4 \cdot \varepsilon_{\mathrm{th}} \cdot \sigma \cdot T^{3}
\end{aligned}
$$

\section{Modellaufbau}

Das thermische Modell wird mittels eines Ersatzschaltbildes hergeleitet, wobei der bekannte Zusammenhang aus Elektrotechnik und Thermodynamik herangezogen wird. Die Modellierung basiert auf dem äquivalenten ESB in Abbildung 4-16. Die Temperaturdifferenz $\Delta T$ beschreibt den Temperaturunterschied zwischen der Batteriezelle und ihrer Umgebung. Durch Anwendung des Knotenpunktsatzes kann die Differentialgleichung des ESB hergeleitet werden (Kritzinger, 2018a):

$$
\dot{Q}=\dot{Q}_{\mathrm{R}_{\mathrm{th}}}+\dot{Q}_{\mathrm{C}_{\mathrm{th}}}=\frac{\Delta T}{R_{\mathrm{th}}}+C_{\mathrm{th}} \frac{d \Delta T}{d t}
$$

Durch Umstellen der Formel (4-21) nach $\Delta T$ und Lösen der Differentialgleichung entsteht die Gleichung zur Berechnung der Temperatur in Abhängigkeit von der Zeit: 


$$
\Delta T\left(t_{\mathrm{n}}\right)=\dot{Q} \cdot R_{\mathrm{th}} \cdot\left(1-e^{\frac{-\Delta t}{R_{\mathrm{th}} \cdot C_{\mathrm{th}}}}\right)+\Delta T\left(t_{\mathrm{n}-1}\right) \cdot e^{\frac{-\Delta t}{R_{\mathrm{th}} \cdot C_{\mathrm{th}}}}
$$

Formel (4-22) hat den Vorteil, dass sie sehr einfach aufgebaut ist und in numerischen Simulationsumgebungen wie Matlab implementierbar ist, wodurch die Rechenzeit gegenüber anderen Ansätzen deutlich reduziert werden kann. Die Herausforderung besteht jedoch darin, die Größen des thermischen Widerstands $R_{\text {th }}$ und der thermischen Kapazität $C_{\text {th }}$ so zu bestimmen, dass das komplexe thermische Verhalten einer Batteriezelle bzw. eines Batteriemoduls sowie deren Interaktion mit der jeweiligen Umgebung beschrieben werden können.

Das thermische Modell (Abbildung 4-16) besteht aus zwei Hauptbestandteilen: ein Wärmegenerationsmodell und ein Wärmedissipationsmodell. Im Wärmegenerationsmodell wird der resultierende Wärmestrom in der Zelle unter Verwendung der Formel (4-11) bestimmt. Für das Wärmedissipationsmodell sind aus der Literatur verschiedene Modellierungstiefen für Einzelzellen bekannt. In (Yan, 2014) wird in konzentrierte, eindimensionale und mehrdimensionale Modelle unterschieden ( $\mathrm{Vgl}$. Abbildung 4-17). Zusätzlich sind in der Abbildung der jeweils notwendige Aufwand zur Modellparametrierung (Abstraktionsgrad) sowie die Ergebnisgenauigkeit und Rechendauer gegeben. Für eine detaillierte Erläuterung der Vor- und Nachteile sowie Aufbau und Parametrierung der unterschiedlichen Modelltiefen sei auf die Fachliteratur verwiesen, beispielsweise in (Fleckenstein, 2013; Yan, 2014; Kritzinger, 2018a).

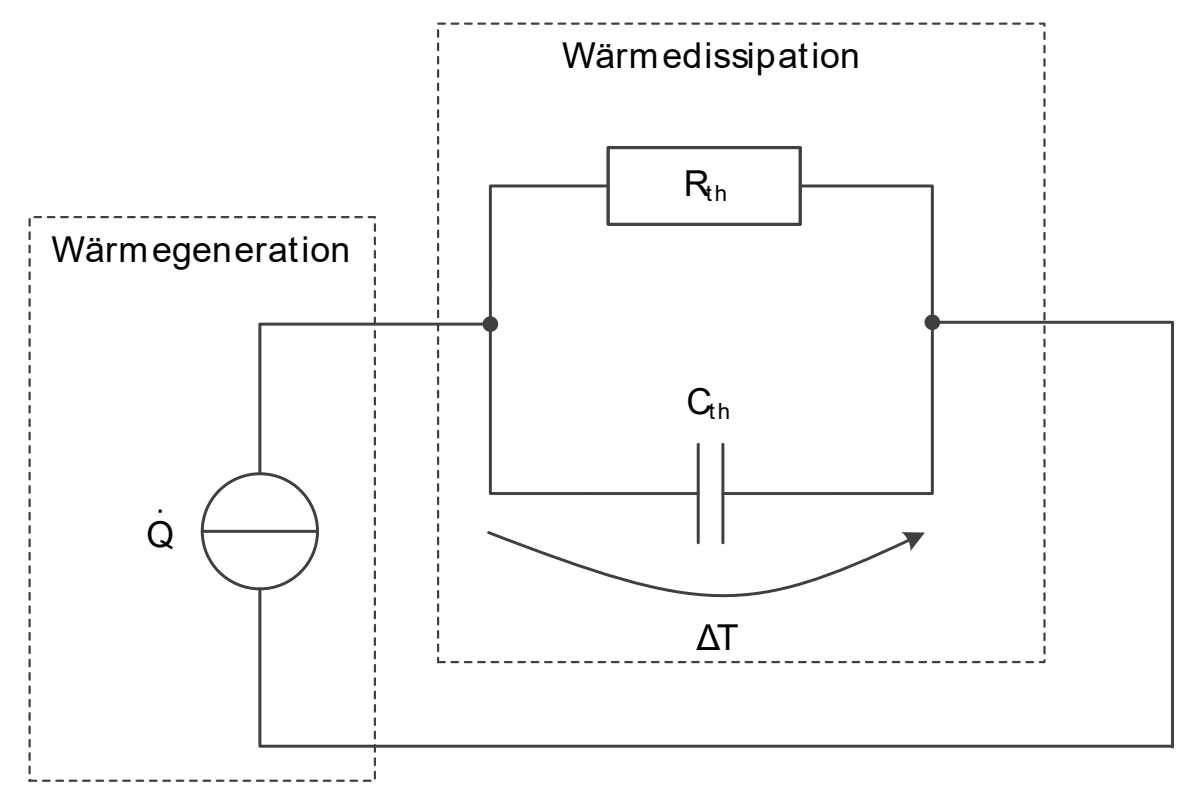

Abbildung 4-16: ESB des thermischen Modells (Kritzinger, 2018a) 
In dieser Arbeit wird aufgrund des niedrigen Aufwands zur Modellparametrierung und der kurzen Rechenzeiten ein konzentrierter Modellansatz zur thermischen Modellierung einer Einzelzelle gewählt. Die Zusammenschaltung mehrerer Zellen zu einem Batteriemodul ist jedoch, anders als bei der elektrischen Modellierung, nicht trivial.

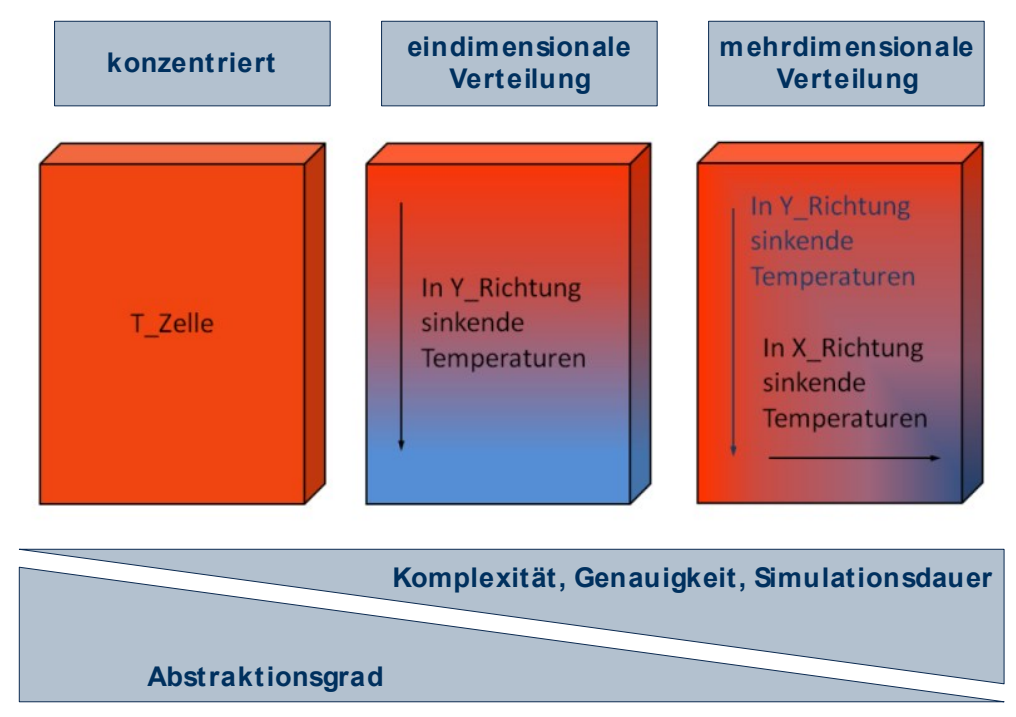

\begin{abstract}
Abbildung 4-17: Temperatur bzw. Temperaturverteilung in konzentrierten, eindimensionalen und mehrdimensionalen Modellen, nach (Yan, 2014)
\end{abstract}

Bei Verwendung des konzentrierten thermischen Modells wird in einen Körper ein Wärmestrom nach Formel (4-11) eingeprägt. Aufgrund der nicht berücksichtigten räumlichen Wärmeverteilung stellt sich gemäß Modellannahme eine homogene Zelltemperatur ein. Bei Betrachtung eines rechteckigen Körpers (prismatische Zellen und Pouchzellen) wird nun unter Berücksichtigung der thermischen Zelleigenschaften ein Teil der eingeprägten Wärme über die 6 Außenflächen des Körpers abgegeben (Kritzinger, 2018b). Es stellen sich demnach 6 Teilwärmeströme ein. Abbildung 4-18 verdeutlicht den Zusammenhang. Darin ist $T_{\mathrm{amb}}$ die Umgebungstemperatur. Zur Bestimmung der 6 Teilwärmeströme aus Abbildung 4-18 werden richtungsabhängige Wärmewiderstände benötigt. Diese werden wie folgt bezeichnet:

$$
R_{\mathrm{th}, \mathrm{x} 1} \text { und } R_{\mathrm{th}, \mathrm{x} 2} \quad R_{\mathrm{th}, \mathrm{y} 1} \text { und } R_{\mathrm{th}, \mathrm{y} 2} \quad R_{\mathrm{th}, \mathrm{z} 1} \text { und } R_{\mathrm{th}, \mathrm{z} 2}
$$

Der Gesamtwiderstand $R_{\text {th }}$ berechnet sich aus der Parallelschaltung der richtungsabhängigen Teilwiderstände (Kritzinger, 2018a).

$$
\frac{1}{R_{\mathrm{th}}}=\frac{1}{R_{\mathrm{th}, \mathrm{x} 1}}+\frac{1}{R_{\mathrm{th}, \mathrm{x} 2}}+\frac{1}{R_{\mathrm{th}, \mathrm{y} 1}}+\frac{1}{R_{\mathrm{th}, \mathrm{y} 2}}+\frac{1}{R_{\mathrm{th}, \mathrm{z} 1}}+\frac{1}{R_{\mathrm{th}, \mathrm{z} 2}}
$$




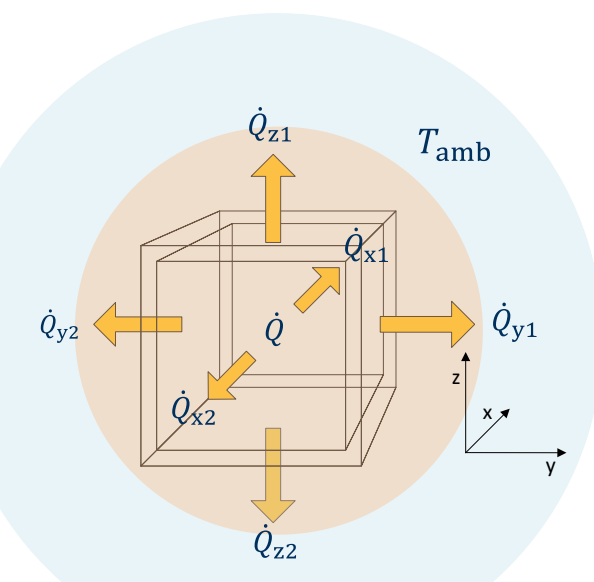

Abbildung 4-18: Rechteckiger Körper mit 6 Abstrahlflächen (Kritzinger, 2018b)

Zur Bestimmung der einzelnen Bestandteile des Gesamtwiderstands müssen die inneren Bestandteile der Zelle und ihre thermischen Eigenschaften berücksichtigt werden. In (Kritzinger, 2018a) werden als wichtige Zellbestandteile für die thermische Modellierung folgende genannt:

- Wickel der Zelle (Separator, Elektrolyt und Elektroden),

- Spalt zwischen Gehäuse und Wickel,

- Zellhülle oder Gehäuse,

- Kühlmedium außerhalb der Zelle.

Alle genannten Bestandteile können anhand des Wärmeübergangs und der Wandstärke als äquivalente thermische Widerstände beschrieben werden. Aufgrund der Verwendung eines konzentrierten Modellansatzes und der damit zentralen Einprägung des Wärmestroms in den Körper werden diese Widerstände in Reihe geschaltet. Für die Richtung $x 1$ ist dies beispielhaft in Abbildung 4-19 dargestellt. Die Zusammensetzung und Berechnung der Teilwiderstände ist in (Kritzinger, 2018a) detailliert beschrieben sowie für die in dieser Arbeit verwendeten Zelle von A123 umgesetzt. Deshalb wird an dieser Stelle auf eine ausführlichere Darstellung verzichtet.

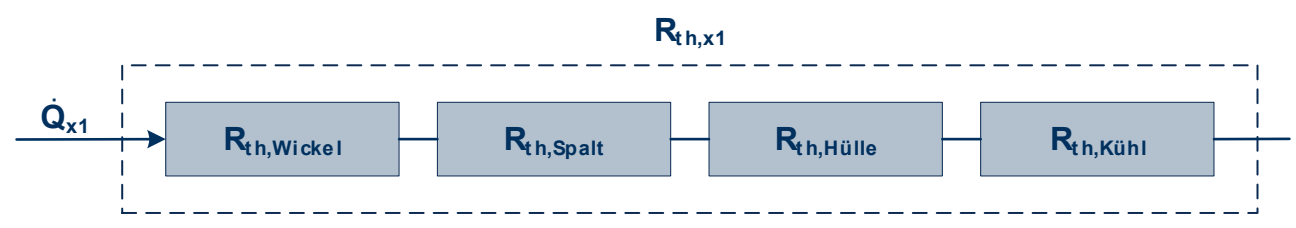
Abbildung 4-19: Summe der thermischen Teilwiderstände in
Richtung x1, nach (Kritzinger, 2018a) 
In Elektrofahrzeugen sind meist mehrere Einzelzellen zu Modulen und diese wiederum zu einem Batteriesystem zusammengeschaltet. In dieser Arbeit wird das thermische Verhalten für die Modulebene bestimmt. Es wird davon ausgegangen, dass die Module untereinander keine gegenseitige thermische Beeinflussung haben. In diesem Modell können Zellen lediglich in $x$-Richtung aneinandergereiht werden. Sind nun mehrere Zellen zu einem Modul zusammengeschaltet, so gibt eine Zelle den Wärmeanteil an der entsprechenden Berührungsfläche nicht an die Umgebung ab, sondern leitet inn in angrenzende Zellen ein. Damit müssen die Widerstände $R_{\mathrm{th}, \mathrm{x} 1}$ und $R_{\mathrm{th}, \mathrm{x} 2}$ angepasst werden, wie in Abbildung 4-20 dargestellt ist.

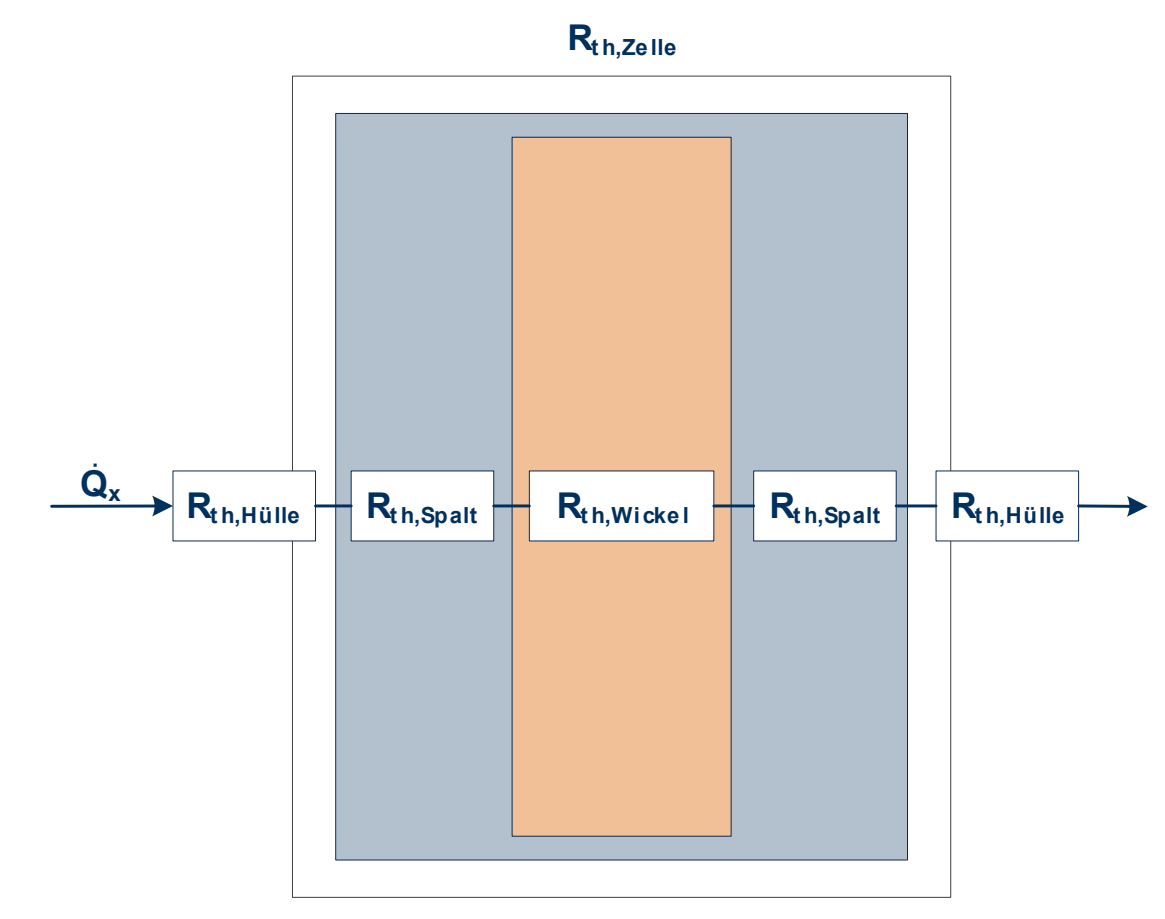

\section{Abbildung 4-20: Thermische Teilwiderstände einer Einzelzelle in} x-Richtung, nach (Kritzinger, 2018a)

In x-Richtung berechnet sich der thermische Widerstand bei Annahme einer symmetrisch aufgebauten Zelle wie folgt (Kritzinger, 2018a):

$$
R_{\mathrm{th}, \mathrm{x}}=2 \cdot\left(R_{\mathrm{th}, \mathrm{Hülle}}+R_{\mathrm{th}, \mathrm{Spalt}}\right)+R_{\mathrm{th}, \text { Wickel }}
$$

Werden mehrere Zellen in einem Modul hintereinander geschaltet, so berechnet sich der thermische Widerstand unter Berücksichtigung der Anzahl serieller Zellen $n_{\text {Zellen }}$ wie folgt (Kritzinger, 2018a):

$$
R_{\mathrm{th}, \mathrm{Modul}, \mathrm{x}}=n_{\text {Zellen }} \cdot R_{\mathrm{th}, \mathrm{x}}
$$


Auf die thermischen Widerstände in y- und z-Richtung hat die Zusammenschaltung von Einzelzellen zu einem Modul keine Auswirkungen, da in diesen Dimensionen keine Stapelung stattfindet und die jeweiligen Außenflächen demnach nicht von anderen Zellen berührt werden.

\section{Kühlung}

Das Thermomanagement der Batterie wird maßgeblich über die Kühlung beeinflusst. Im thermischen Modell können verschiedene Kühlungsarten simuliert werden. Dies sind im Wesentlichen:

- Passive Luftkühlung,

- Aktive / forcierte Luftkühlung,

- Wasserkühlung.
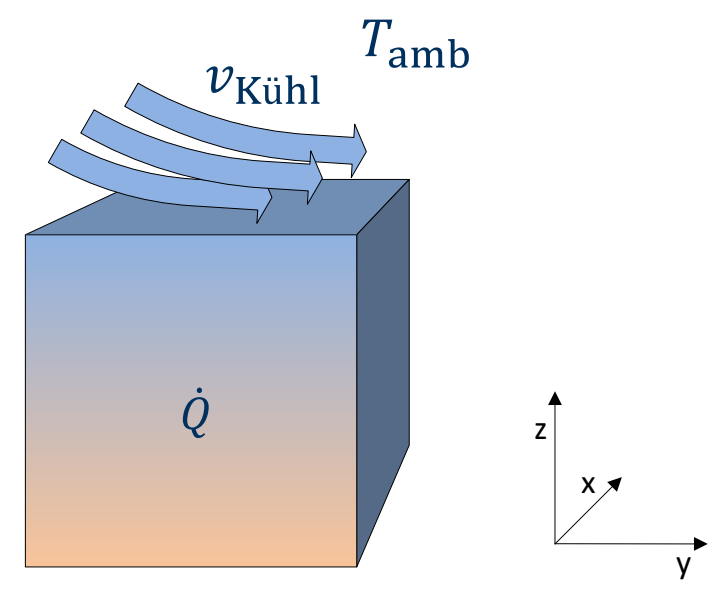

Abbildung 4-21: Modellierung von Kühlstrategien (Kritzinger, 2018b)

Tabelle 4-6: Übersicht konvektive Wärmeübergänge für verschiedene Kühlungsmechanismen (Kritzinger, 2018a)

\begin{tabular}{lcc}
\hline $\begin{array}{l}\text { Kühlungs- } \\
\text { mechanismus }\end{array}$ & $\begin{array}{c}\text { Anström- } \\
\text { geschwindigkeit }\left(\frac{\boldsymbol{m}}{\boldsymbol{s}}\right)\end{array}$ & $\begin{array}{c}\text { Wärmeübergangs- } \\
\text { koeffizient }\left(\frac{\boldsymbol{W}}{\boldsymbol{m}^{\mathbf{2}} \cdot \boldsymbol{K}}\right)\end{array}$ \\
\hline Luftkühlung passiv & 0 & 10 \\
Luftkühlung aktiv & 30 & 100 \\
Wasserkühlung & 0,5 & 2000 \\
& 1,0 & 4000 \\
\hline
\end{tabular}


Die Kühlung wird über den konvektiven Widerstand $R_{\text {th,kühl }}$ aus Abbildung 4-19 modelliert. Verschiedene Kühlungsarten können über die Variation des Wärmeübergangskoeffizienten $\alpha_{\mathrm{k}}$ aus Formel (4-13) abgebildet werden. Dieser wird in Abhängigkeit der Anströmgeschwindigkeit $v_{\text {Kühl }}$ für jede Außenfläche individuell gewählt. Abbildung 4-21 zeigt den prinzipiellen Zusammenhang. In (Kritzinger, 2018a) sind für verschiedene Anströmgeschwindigkeiten entsprechende Wärmeübergangskoeffizienten gegeben (Vgl. Tabelle 4-6).

\title{
Modellvalidierung auf Zellebene
}

Das thermische Modell wird zunächst für eine Einzelzelle validiert. Dafür wurde ein dynamisches Stromprofil (Vgl. Anhang C.1) in die Zelle eingeprägt. Die Zelle befand sich während der Messung in einer Klimakammer, wobei die Umgebungstemperatur auf konstant $50^{\circ} \mathrm{C}$ gehalten wurde. Die Messung der Zelltemperatur wurde mittels eines Temperatursensors mittig auf der Zelloberfläche (Vgl. Abbildung 4-22) gemessen. ${ }^{13}$

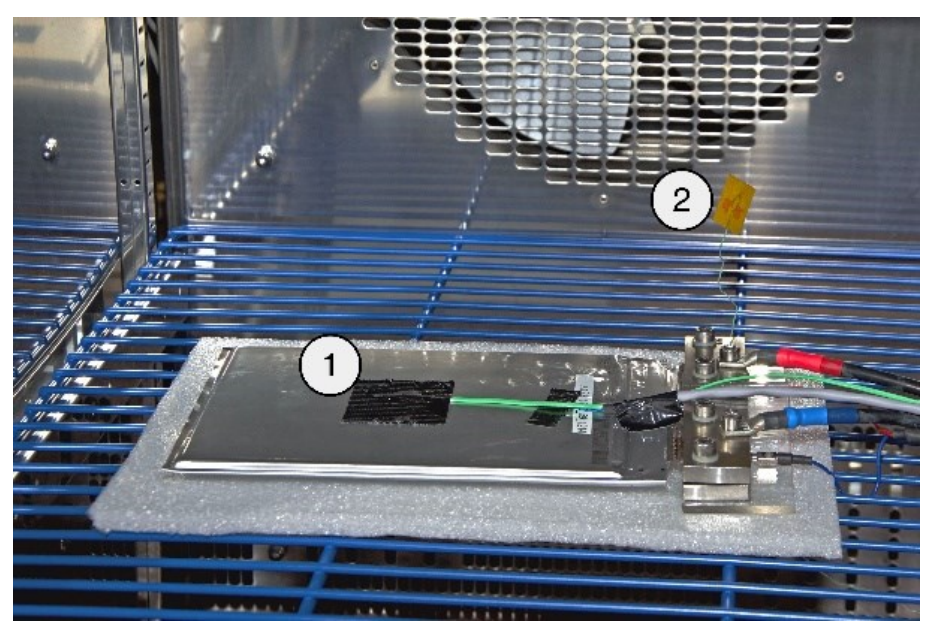

\begin{abstract}
Abbildung 4-22: Versuchszelle mit zwei Temperatursensoren, 1) Oberflächentemperatur, 2) Umgebungstemperatur (Richter, 2017)
\end{abstract}

In Abbildung 4-23 (oben) sind die Temperaturverläufe der Simulation und der Messung vergleichend dargestellt. Der Vergleich der Verläufe zeigt eine sehr gute Übereinstimmung von Messung und Simulation. In Abbildung 4-23 (unten) ist die Differenz aus Messung und Simulation dargestellt. In Tabelle 4-7 sind charakteristische Kennwerte zum Vergleich gegeben. Daraus geht hervor, dass die maximale Abweichung

\footnotetext{
${ }^{13}$ Das Modell berechnet die Temperatur im Inneren der Zelle. Eine Messung der Zellinnentemperatur ist jedoch ohne irreversible Zellschädigung nicht möglich. Deshalb wird im Rahmen dieser Arbeit die Oberflächentemperatur als Zelltemperatur verwendet.
} 
$+0,26^{\circ} \mathrm{C}$ bzw. $-0,09^{\circ} \mathrm{C}$ beträgt. Mittels Vergleichs der Effektivwerte aus Abweichung und gemessenem Signal ergibt sich ein relativer Modellfehler von 0,24\%.
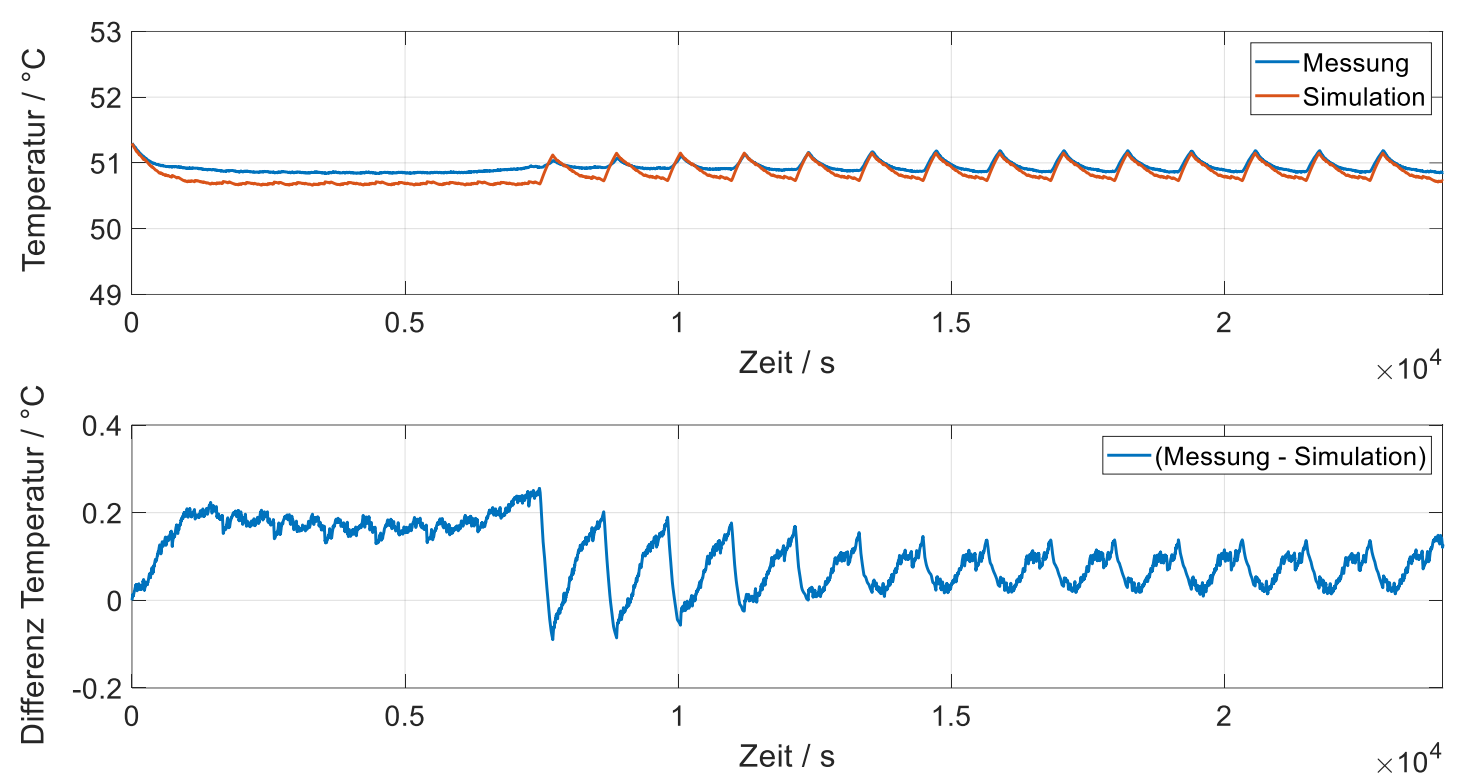

Abbildung 4-23: Validierungsergebnis thermisches Modell auf Zellebene

Tabelle 4-7: Zusammenfassung der Validierungsergebnisse der thermischen Modellbildung (Zellebene)

\begin{tabular}{lc}
\hline & Temperaturprofil \\
\hline max. Abweichung & $+0,26^{\circ} \mathrm{C}$ \\
(absoluter Fehler) & $-0,09^{\circ} \mathrm{C}$ \\
RMS-Wert Abweichung & $0,12^{\circ} \mathrm{C}$ \\
RMS-Wert gemessenes & $50,9^{\circ} \mathrm{C}$ \\
Profil & $0,24 \%$ \\
relativer Fehler & \\
\hline
\end{tabular}

\section{Modellvalidierung auf Batterieebene}

Die Validierung auf Batterieebene wird anhand eines Hochleistungsbatteriespeichers aus einem Formula Student Electric Rennwagen durchgeführt. Die Batterie besteht dabei aus fünf Stacks, in welchen jeweils 56 Zellen integriert sind, und wird bei Bedarf 
über Lüfter aktiv gekühlt. Der Aufbau der Batterie ist in (Gatter, 2019) ausführlich beschrieben. Für die Validierung wird der gemessene Batteriestrom (Vgl. Anhang C.2) als Eingangsgröße für das thermische Modell verwendet. In Abbildung 4-24 sind die Ergebnisse der Messung und der Simulation vergleichend dargestellt.
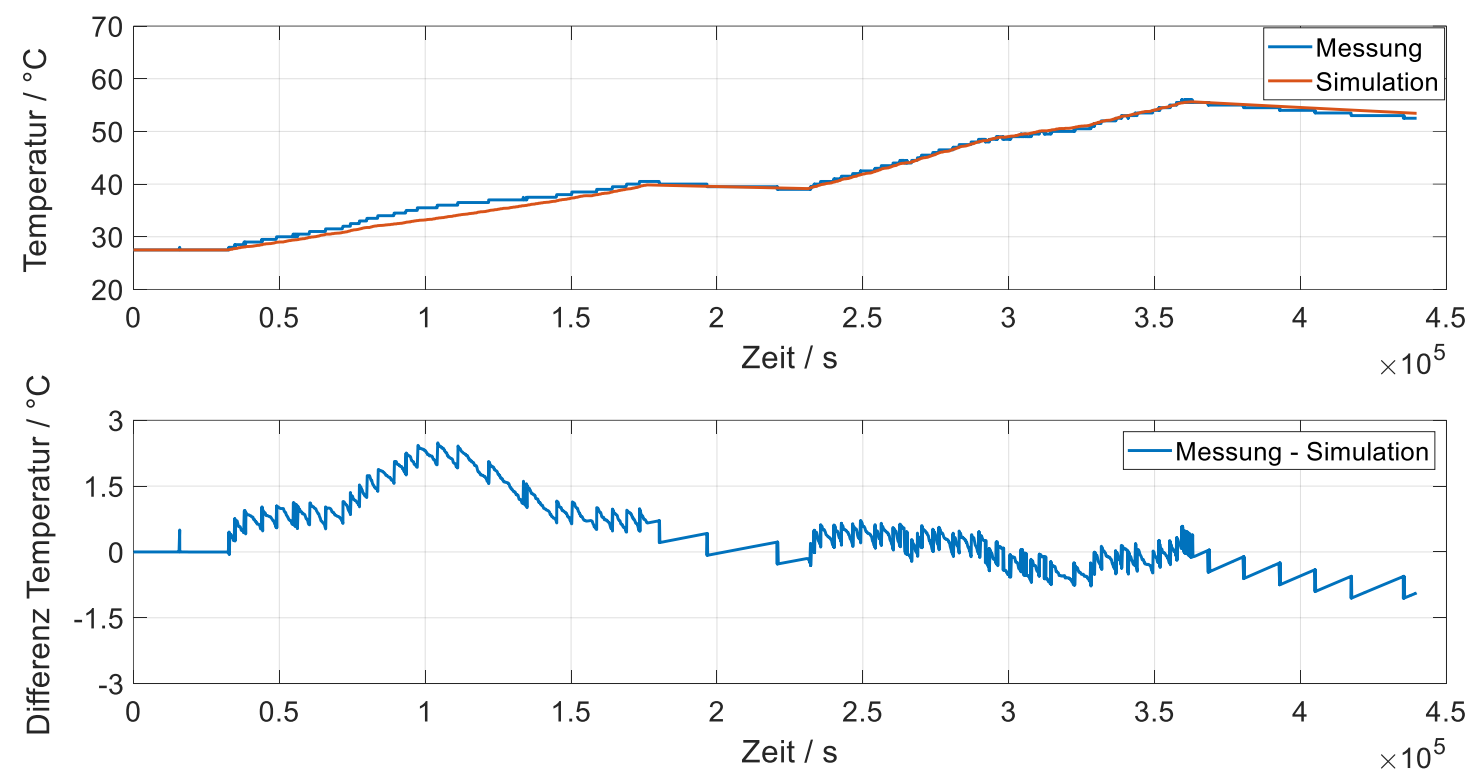

Abbildung 4-24: Validierungsergebnis thermisches Modell auf Batterieebene, Daten aus (Gatter, 2019)

Tabelle 4-8: Zusammenfassung der Validierungsergebnisse der thermischen Modellbildung (Batterieebene)

\begin{tabular}{lc}
\hline & Temperaturprofil \\
\hline max. Abweichung & $+2,49^{\circ} \mathrm{C}$ \\
(absoluter Fehler) & $-1,06^{\circ} \mathrm{C}$ \\
RMS-Wert Abweichung & $0,86 \mathrm{~A}$ \\
RMS-Wert gemessenes & $43,01 \mathrm{~A}$ \\
Profil & $1,99 \%$ \\
relativer Fehler & \\
\hline
\end{tabular}

In Tabelle 4-8 sind charakteristische Kennwerte zum Vergleich gegeben. Daraus geht hervor, dass die maximale Abweichung $+2,49^{\circ} \mathrm{C}$ bzw. $-1,06^{\circ} \mathrm{C}$ beträgt. Mittels Vergleichs der Effektivwerte aus Abweichung und gemessenen Signal ergibt sich ein 
relativer Modellfehler von 1,99\%. Dieser liegt im Vergleich zur Validierung auf Zellebene deutlich höher. Als eine mögliche Ursache können sowohl eine ungenaue Parametrierung der Wärmeübergänge zwischen den äußeren Zellen und dem Gehäuse der Stacks als auch die Parametrierung der Kühlung ausgemacht werden. Die Drehzahl des Lüfters und damit die Kühlleistung werden nicht aufgezeichnet. Aufgrund der bedarfsgerechten Steuerung liegt somit keine konstante Kühlung der Batterie während der Belastung vor. Auffällig ist die große Abweichung im Bereich um 10.000 s. An dieser Stelle steigt die gemessene Temperatur überproportional an, während die Simulation einen konstanten Anstieg im Bereich zwischen $400 \mathrm{~s}$ und $17.000 \mathrm{~s}$ aufweist. Dies wurde in (Gatter, 2019) analysiert, jedoch konnte keine Ursache ermittelt werden. Aus Abbildung 4-24 geht jedoch auch hervor, dass es sich um keinen fortführenden Fehler handelt, da die maximale Abweichung im weiteren Signalverlauf wieder abnimmt.

\subsubsection{Alterungsmodell}

Li-lonen Batterien sind einem permanenten Alterungsprozess ausgesetzt, welcher durch ständig ablaufende chemische Prozesse innerhalb der Zellen hervorgerufen wird. Einen guten Überblick über die einzelnen Alterungsmechanismen und die daraus resultierenden Effekte sind unter anderem in (Petersohn, 2012) gegeben. In (Fleckenstein, 2013) sind verschiedene Möglichkeiten zur Modellbildung des Alterungsverhaltens von Li-lonen-Zellen aufgeführt:

- Elektrochemische Modelle,

- Empirische Modelle.

Ein weit verbreitetes empirisches Alterungsmodell ist das Ladungsdurchsatzmodell (Fleckenstein, 2013). Es erlaubt eine detaillierte Betrachtung einzelner Alterungseinflussfaktoren, kann (bei entsprechender Parametrierung) auf verschiedenste Zelltypen angewendet werden und soll in dieser Arbeit zur Anwendung kommen.

\section{Grundlagen}

Hauptverantwortlich für die Alterung einer Li-Ionen Zelle ist die "Solid Electrolyte Interphase" (SEI). Dabei handelt es sich um eine Passivschicht, welche sich zwischen Anode und Elektrolyt ausbildet (Abbildung 4-1). Diese Schicht unterliegt einem ständigen Entwicklungsprozess. In einer fabrikneuen Zelle ist zunächst keine SEI ausgebildet. Dies geschieht erstmalig in der Formierungsphase während der ersten Zyklisierungen ${ }^{14}$. Für die Bildung der SEI wird stets aktives Lithium verbraucht, wodurch

\footnotetext{
${ }^{14}$ Als Zyklisierung wird das Laden und Entladen einer Zelle bzw. einer Batterie bezeichnet.
} 
die nutzbare Kapazität der Zelle sinkt. Hohe Temperaturen, hohe Ströme oder große SOC-Hübe können die SEI beschädigen. Die Neuausbildung verbraucht ebenfalls aktives Lithium. Die SEl ist einerseits elektrisch isolierend, jedoch andererseits leitfähig für Lithium-Ionen. Somit wirkt eine Verdickung der SEI mit zunehmendem Ladungsdurchsatz als ein zusätzliches Hindernis, was zu einem erhöhten Innenwiderstand der Zelle führt (Fischhaber et al., 2016).

Die beiden genannten Phänomene der Batteriealterung, Abnahme der Kapazität und Anstieg des Innenwiderstands, werden typischerweise zur Quantifizierung der Alterung herangezogen. Eine reduzierte Kapazität der Zellen wirkt sich in Form einer geringeren Menge entnehmbarer Energie aus, was bei Elektrofahrzeugen im Wesentlichen einer Reduzierung der Reichweite entspricht. Durch einen Anstieg des Innenwiderstands verliert die gesamte Batterie zunehmend an Leistungsfähigkeit. Dies wirkt sich derart aus, dass hohe Fahrleistungen u.U. nicht mehr realisierbar sind (vermindertes Beschleunigungsvermögen und reduzierte Höchstgeschwindigkeit) sowie hohe Ladeleistungen nicht mehr erreicht werden können (längere Ladezeiten für gleiche Energiemengen). Zwei oftmals in der Literatur verwendete Kriterien, welche auch in dieser Arbeit angewendet werden, definieren das End-of-Life (EOL) der Batterie wie folgt:

- Reduzierung der nutzbaren Kapazität auf 80\% der Nennkapazität,

- Verdopplung des Zellinnenwiderstands auf $200 \%$.

Zum Erreichen des EOL muss nur eines der beiden Kriterien erfüllt sein. Grundsätzlich gilt, dass die Zellen bei Erreichen der Kriterien nicht physisch defekt sind, sondern noch weiterverwendet werden können. Jedoch ist allgemein davon auszugehen, dass die Batterie dann nicht mehr den hohen Leistungsanforderungen in Elektrobussen genügen und entsprechend auszutauschen sind. Einer Weiterverwendung in Second-LifeAnwendungen (z.B. als stationäre Speicher) ist jedoch in vielen Fällen möglich (Fischhaber et al., 2016).

Die Alterung von Li-lonen Batterien ist ein kontinuierlich voranschreitender Prozess, welcher in zwei Anteile unterteilt werden kann:

- Kalendarische Alterung (bei unbelasteter Batterie), und

- Zyklische Alterung (bei belasteter Batterie).

Eine Li-lonen Batterie altert auch, wenn sie nicht in Betrieb und damit unbelastet ist (z.B. während der Fahrzeugabstellung). Dieser Anteil des Alterungsprozesses beginnt streng genommen direkt nach dem Ende der Zellproduktion. Die kalendarische Alterung ist eine Funktion in Abhängigkeit der Zeit. Gemäß dem Stand der Technik können zwei wesentliche Einflussfaktoren auf den kalendarischen Alterungsprozess definiert werden (Jossen et al., 2019): 
- Lagerungstemperatur, und

- Prozentualer Ladezustand der Zelle während der Lagerung (SOC - State of Charge).

Die zyklische Alterung ist eine Funktion in Abhängigkeit des Ladungsdurchsatzes der Batterie, welcher in Amperestunden (Ah) angegeben wird. Sie beschreibt demnach den Alterungsprozess aufgrund der Zyklisierung. Gemäß dem Stand der Technik können vier wesentliche Einflussfaktoren auf den zyklischen Alterungsprozess definiert werden (Jossen et al., 2019):

- Batterietemperatur während der Zyklisierung,

- Obere und untere Grenze des Ladezustands zu Beginn und am Ende der Zyklisierung (SOC-Hub bzw. $\triangle \mathrm{SOC}$ )

- Höhe des Batteriestroms (C-Rate),

- Mittlerer Ladezustand der Batterie (SOC) während der Zyklisierung.

Abbildung 4-25 fasst die bisherigen Aussagen zur Thematik der Alterung von Li-lonen Batterien zusammen.

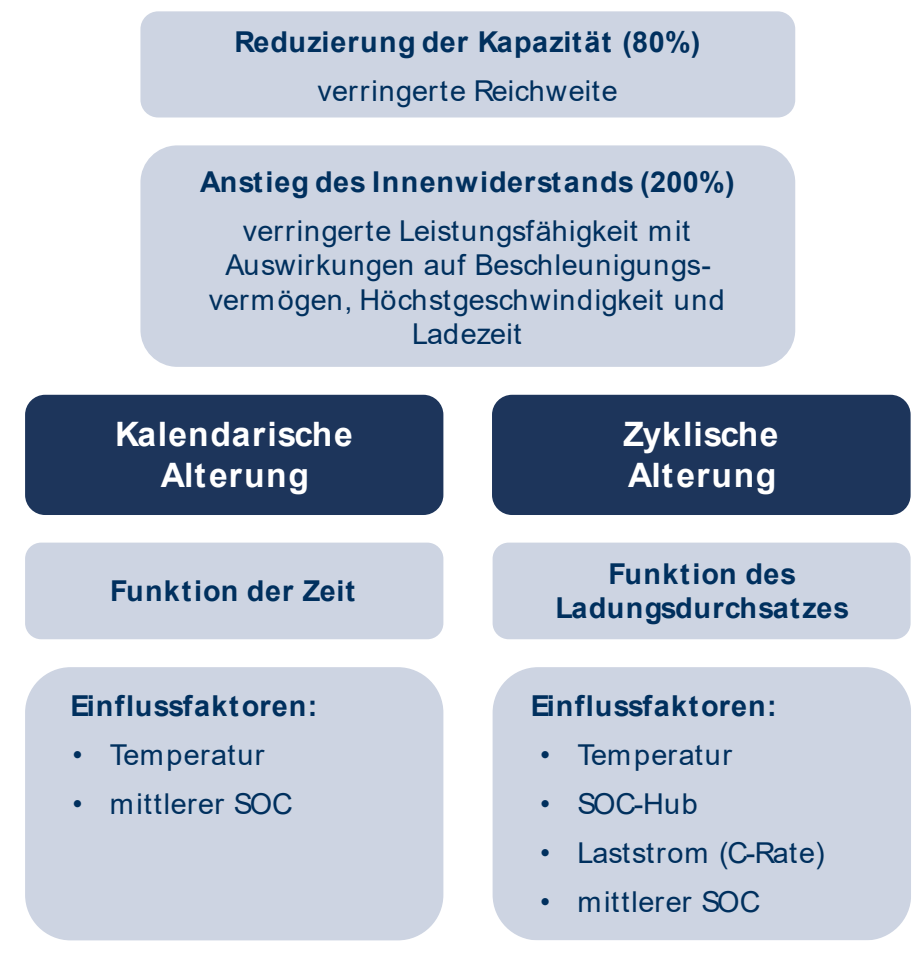

Abbildung 4-25: Übersicht zur Alterung von Li-lonen Batterien 
Für die Erstellung und Parametrierung des benötigten Alterungsmodells ist es erforderlich, dass die Einflussfaktoren qualifiziert und quantifiziert werden. Alterungsmessungen von Batterien sind grundsätzlich sehr zeitintensiv. Bei einer vollständigen Vermessung bis zum Erreichen eines der EOL-Kriterien können $u$. U. mehrere Jahre vergehen, wenn die Umgebungs- und Belastungsbedingungen den realen Einsatzbedingungen entsprechen. Deshalb kommen zur Bestimmung des Alterungsverhaltens oftmals beschleunigte Alterungstests zum Einsatz. Dabei werden die zu untersuchenden Zellen unter Laborbedingungen einer erhöhten Belastung unterzogen, um anschließend mittels Inter- und Extrapolation Rückschlüsse auf reale Einsatzszenarien ziehen zu können. Zur empirischen Modellbildung und Parametrierung eines vollständigen Alterungsmodells - sprich unter Berücksichtigung aller oben genannten Einflussfaktoren - ist eine entsprechend große Testmatrix erforderlich. Die Testmatrix für die Modellierung in dieser Arbeit wurde bereits in (Meubrink, 2016) erstellt und die dazugehörigen Messungen gestartet.

Die Testmatrix ist nach dem „One Factor at the time" Prinzip aufgebaut. Danach wird stets nur ein Einflussfaktor variiert, während alle anderen Faktoren konstant gehalten werden. Somit kann sichergestellt werden, dass die jeweilige Schädigungswirkung eines Faktors isoliert und von anderen Einflussfaktoren unbeeinflusst bestimmt und beurteilt werden kann. Eine Übersicht der einzelnen Messreihen der Testmatrix zur Bestimmung der zyklischen Alterung ist in Tabelle 4-9, die Messreihen der kalendarischen Alterung sind in Tabelle 4-10 gegeben. Zur Ermittlung des quantitativen Verlaufs eines Einflussfaktors werden jeweils drei Stützstellen (Arbeitspunkte) vermessen, um auch nichtlineare Zusammenhänge erkennen und abbilden zu können. In Zeile 2 der Tabelle 4-9 ist die Messreihe zur Variation des Einflussfaktors mittlerer SOC angegeben. Die in (Meubrink, 2016) aufgestellte Messmatrix beinhaltet keine vollständige Messreihe zur Bestimmung der Schädigungswirkung des mittleren SOCs bei zyklischer Belastung, weshalb der Einflussfaktor in dieser Arbeit nicht berücksichtigt werden kann. Eine Übernahme der Messreihe aus der kalendarischen Alterungsmatrix ist aufgrund der unterschiedlichen Bezugsgrößen Zeit und Ladungsdurchsatz nicht möglich.

Die Zellen zur Bestimmung der zyklischen Alterung werden entsprechend den gesetzten Rand- und Umgebungsbedingungen mit Rechteck-Stromprofilen kontinuierlich zyklisiert. Die Zyklisierung wird in regelmäßigen Abständen unterbrochen, um in einem Zwischentest ${ }^{15}$ den Fortschritt der Alterung zu bestimmen. Dafür wird für alle Zellen (zyklisch und kalendarisch) ein Kapazitätstest bei $25^{\circ} \mathrm{C}$ Umgebungstemperatur durchgeführt, wobei diese einmal vollständig entladen und wieder geladen werden.

\footnotetext{
15 Ein Zwischentest wird auch als Reference Performance Test (RPT) bezeichnet. Der Ablauf eines RPTs ist in (Richter, 2017) ausführlich beschrieben.
} 
Des Weiteren wird der Innenwiderstand der Zellen ebenfalls bei $25^{\circ} \mathrm{C}$ sowie einem SOC von $50 \%$ gemessen.

Tabelle 4-9: Messreihen zur Modellbildung (zyklische Alterung)

\begin{tabular}{llll}
\hline Temperatur & SOC & SSOC & C-Rate \\
\hline $\mathrm{T}_{1} / \mathrm{T}_{2} / \mathrm{T}_{3}$ & konstant & konstant & konstant \\
konstant & $\mathrm{SOC}_{1} / \mathrm{SOC}_{2} / \mathrm{SOC}_{3}$ & konstant & konstant \\
& & $\Delta \mathrm{SOC}_{1} / \Delta \mathrm{SOC}_{2} /$ & konstant \\
konstant & konstant & $\Delta \mathrm{SOC}_{3}$ & \\
& & & C-Rate $/$ C-Rate \\
& konstant & konstant & C-Rate \\
\hline
\end{tabular}

Tabelle 4-10: Messreihen zur Modellbildung (kalendarische Alterung)

\begin{tabular}{ll}
\hline Temperatur & SOC \\
\hline $\mathrm{T}_{1} / \mathrm{T}_{2} / \mathrm{T}_{3}$ & konstant \\
konstant & $\mathrm{SOC}_{1} / \mathrm{SOC}_{2} / \mathrm{SOC}_{3}$ \\
\hline
\end{tabular}

\section{Auswertung der Messreihen und Ermittlung von Schädigungsfunktionen}

Die Auswertung der Messreihen dient der Vorbereitung der Modellbildung sowie der Parametrierung des Alterungsmodells und erfolgt zweistufig. Zunächst erfolgt eine Analyse der generellen Verläufe für die Abnahme der Kapazität und den Anstieg des Innenwiderstands über den unabhängigen Variablen Ladungsdurchsatz (zyklisch) und Zeit (kalendarisch). Dafür werden für die gemessenen Kapazitäts- und Innenwiderstandswerte Regressionsfunktionen mittels einer Fitting-Funktion berechnet. AnschlieBend sollen aus den jeweils drei zur Verfügung stehenden Stützpunkten eines Einflussfaktors sogenannte Schädigungsfunktionen ermittelt werden. In Abbildung 4-26 bis Abbildung 4-29 (jeweils links) sind die Ergebnisse der Messreihen nach Tabelle 4-9 und Tabelle 4-10 gezeigt. Aus allen Messreihen ist deutlich erkennbar, dass sowohl die Abnahme der Kapazität als auch der Anstieg des Innenwiderstands einen annähernd linearen Verlauf der Schädigung über der Zeit (kalendarisch) bzw. über dem Ladungsdurchsatz (zyklisch) aufweisen. Im rechten Teil der Abbildungen sind jeweils die normierten Schädigungsfunktionen aufgetragen, welche aus den Anstiegen der 
Regressionsgeraden ermittelt wurden. Die Auswertung der Messreihen erfolgt getrennt nach kalendarischer und zyklischer Alterung.

In Abbildung 4-26 sind die Messreihen und die Schädigungsfunktionen der Temperatur der zyklischen Alterung abgebildet. Sowohl die Kapazität als auch der Innenwidertand weisen einen exponentiellen Anstieg auf. Dieser Zusammenhang wird in der Literatur oftmals mit der Arrhenius-Gleichung und der daraus resultierenden 10K-Regel begründet. Demnach halbiert sich aufgrund schneller ablaufender Reaktionsprozesse die Lebensdauer einer Li-Ionen Zelle bei einer Steigerung der Temperatur um $10 \mathrm{~K}$ (Richter, 2017).
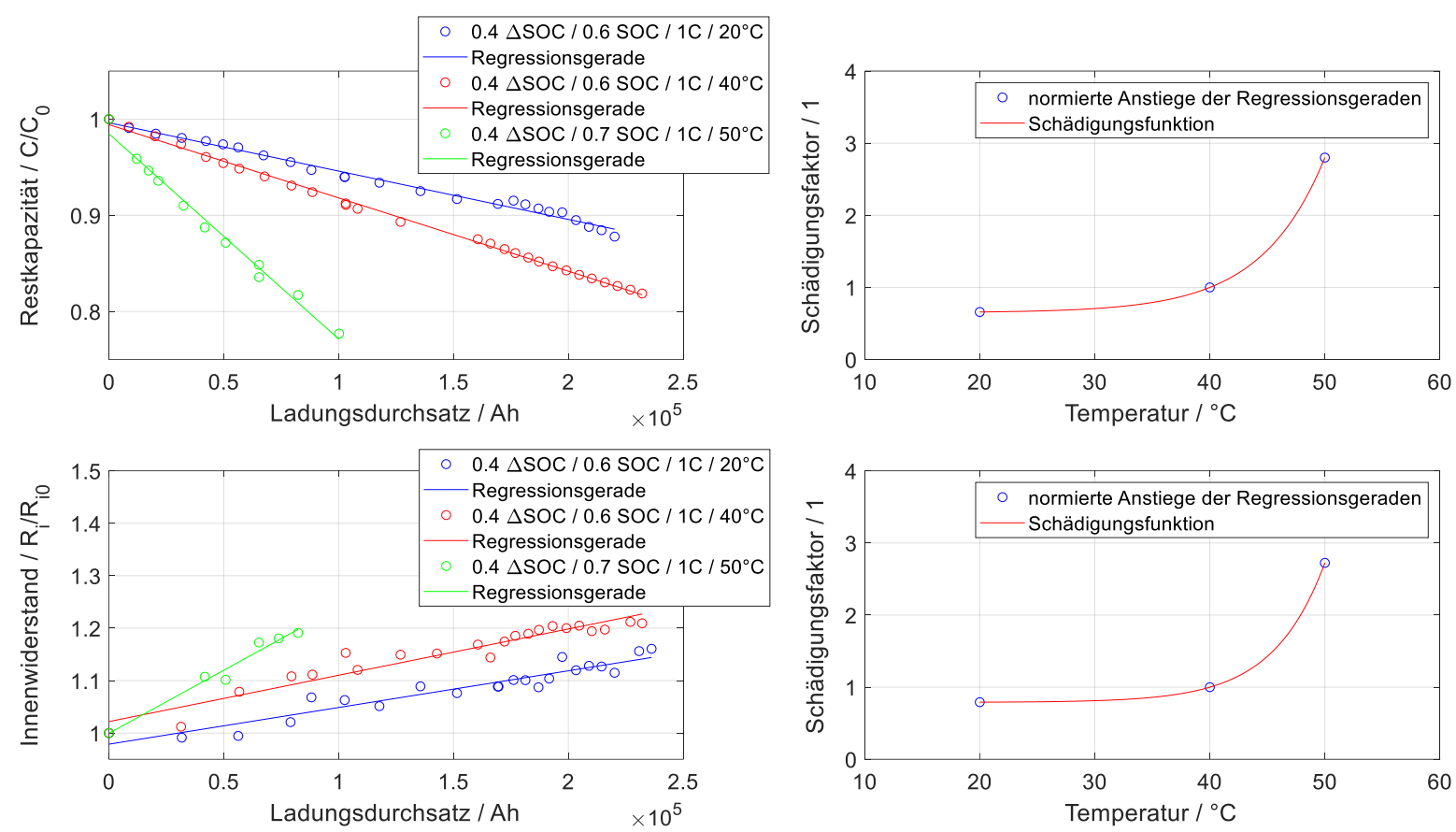

\section{Abbildung 4-26: Messergebnisse Einflussfaktor Temperatur (zyklisch) (links), resultierende Schädigungsfunktionen (rechts)}

In Abbildung 4-27 sind die Messreihen und die Schädigungsfunktionen der Temperatur der kalendarischen Alterung abgebildet. Für die Kapazität ergibt sich analog zur zyklischen Alterung eine gut gefittete e-Funktion.

In Abbildung 4-28 sind die Messreihen und die Schädigungsfunktionen des $\mathbf{\Delta S O C s}$ abgebildet. Die Kapazität weist einen exponentiellen Anstieg auf. Eine Vergrößerung des SOC-Hubs führt demnach zu einer überproportionalen Schädigung der Batterie. Bei der Auswertung des Innenwiderstands fällt hingegen auf, dass bei $\Delta S O C=0,2$ eine größere Schädigung gemessen wurde als bei $\triangle S O C=0,4$. Aus der Literatur ist ebenfalls ein exponentieller Anstieg der Schädigung mit steigendem $\triangle \mathrm{SOC}$ bekannt, beispielsweise aus (Omar et al., 2014). Es wird deshalb auch für den Innenwiderstand eine 
exponentielle Funktion gefittet. Eine mögliche Begründung der Abweichung zwischen den Punkten $\triangle S O C=0,2$ und $\triangle S O C=0,4$ liegt im langen Versuchszeitraum und den zahlreichen Zwischentests der Messreihe, was mutmaßlich zu Messungenauigkeiten geführt hat. Für die Vermessung des Innenwiderstands wurde das Messsystem BT 3562 des japanischen Herstellers Hioki verwendet. Dabei werden die Messkanäle über Klemmen händisch an den Kontaktierungsflächen der Zellen befestigt. Hierbei ist eine möglichst gleichmäßige Positionierung der Klemmen während aller Messungen von besonderer Wichtigkeit, da bereits bei sehr kleinen Abweichungen unterschiedliche Ergebnisse erzeugt werden können.
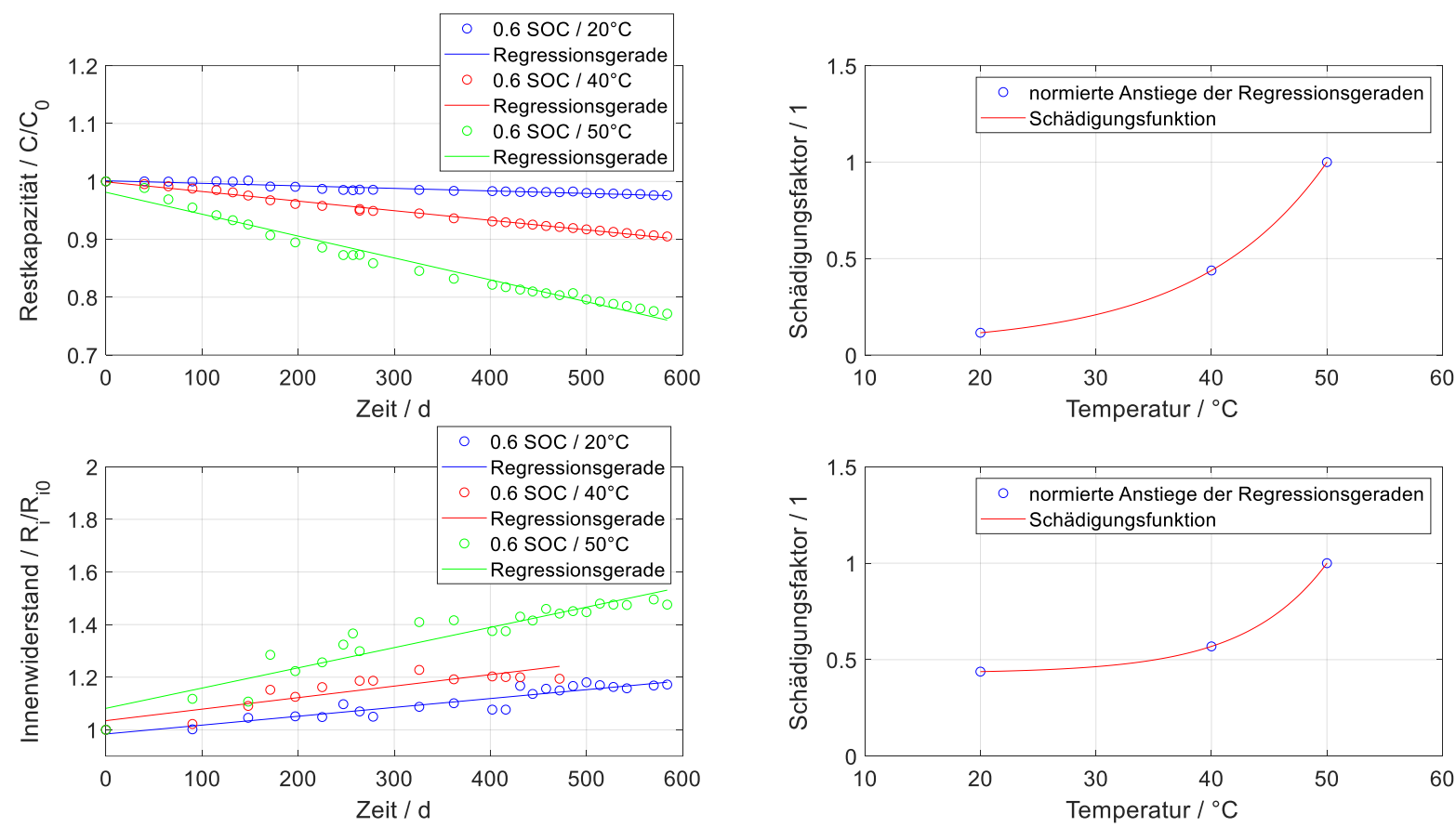

\section{Abbildung 4-27: Messergebnisse Einflussfaktor Temperatur (kalendarisch) (links), resultierende Schädigungsfunktionen (rechts)}

In Abbildung 4-29 sind die Messreihen und die Schädigungsfunktionen der C-Rate abgebildet. Aus der Literatur ist bekannt, dass die Schädigung mit steigender C-Rate zunimmt (z.B. (Herb, 2010)). Eine höhere Stromdichte bedingt eine größere Volumenarbeit in der Zelle, was dazu führt, dass die SEl-Schicht durch kleine Risse beschädigt wird, welche dann unter Verwendung von aktivem Lithium neu ausgebildet wird (Fischhaber et al., 2016). Dieser Zusammenhang einer stärkeren Alterung bei steigender C-Rate ist in der Messreihe in Abbildung 4-29 jedoch weder bei der Kapazität noch beim Innenwiderstand erkennbar. Vielmehr geht aus der Abbildung hervor, dass die Zelle mit der kleinsten C-Rate die prozentual größte Schädigung aufweist. Dementsprechend haben die resultierenden Schädigungsfunktionen einen negativen Anstieg. 
Das heißt, dass die Schädigung mit steigender C-Rate abnimmt. Eine mögliche Erklärung dafür liefert der Vergleich der jeweils umgesetzten Ladungsmenge als Funktion der Zeit, welche in Abbildung 4-30 dargestellt ist. Es ist erkennbar, dass für die Zelle mit 0,5 C Belastung zur Umsetzung der gleichen Ladungsmenge deutlich mehr Versuchstage erforderlich sind als für die Zellen mit 1,0 C bzw. 1,5 C Belastung. Dabei wird die zyklische Alterung von der kalendarischen Alterung offenbar derart überlagert, dass sie diese sogar in geringem Maße überwiegt. Dieser Effekt wurde auch in (Fleckenstein, 2013) beobachtet. Als Folge weist die Schädigungsfunktion der C-Rate für Kapazität und Innenwiderstand in dieser Quelle ebenfalls einen negativen Anstieg auf. Dieser wird im Alterungsmodell entsprechend parametriert, sodass keine Korrektur der Zyklisierungszeit erfolgt. Die negativen Anstiege der Funktionen sind dabei nicht als alterungshemmend oder gar als Verzögerung, sondern als eine Art Korrekturfaktor zur Beschreibung des Zusammenhangs aus Ladungsdurchsatz und Versuchsdauer, zu verstehen. Beide Schädigungsfunktionen werden als exponentielle Funktionen gefittet. Somit ist sichergestellt, dass für sehr hohe C-Raten keine negativen Schädigungsfaktoren errechnet werden können, welche dann gemäß Formel (4-31) und (4-32) zu einer "positiven" Schädigung führen würden.
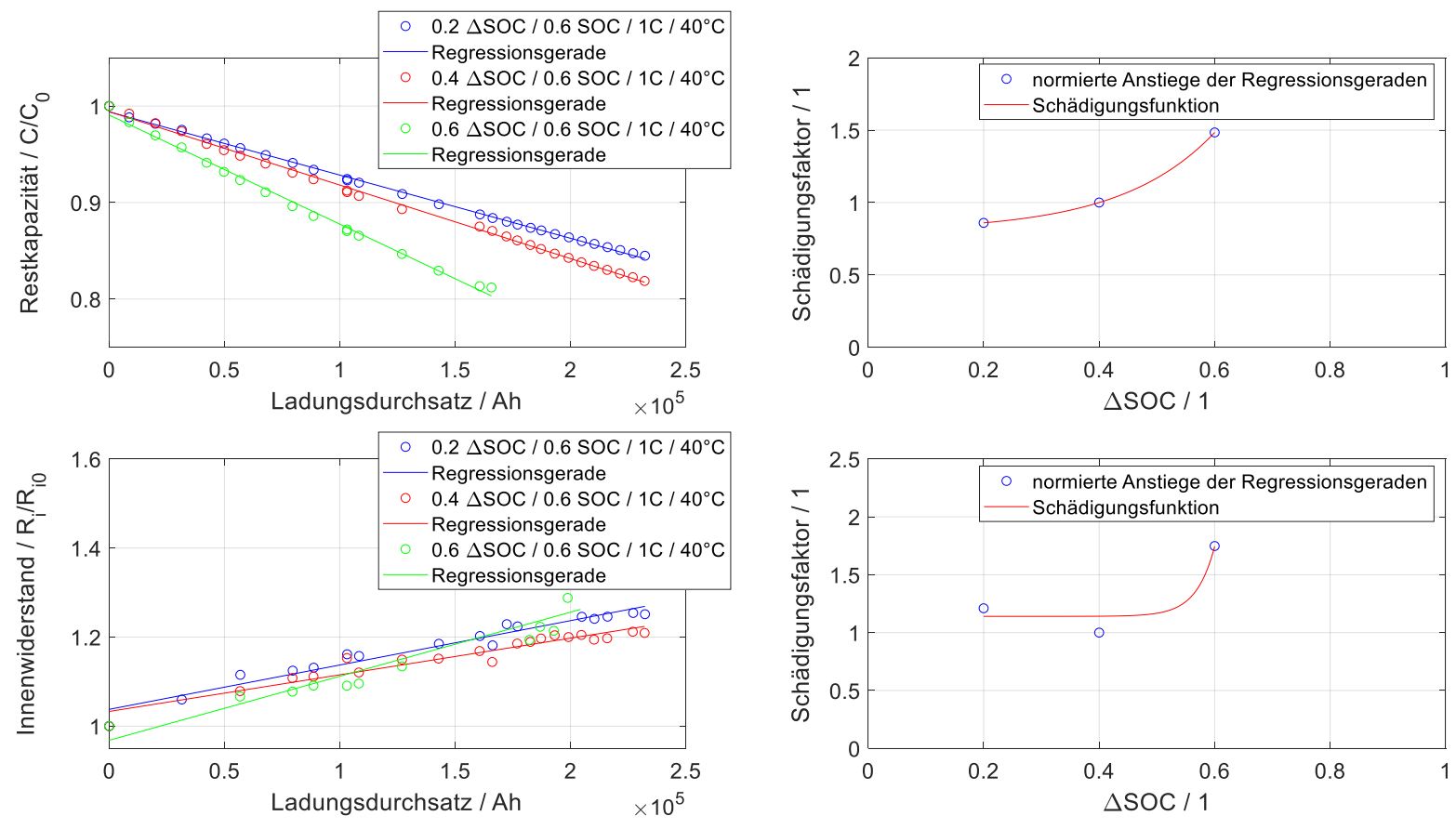

\footnotetext{
Abbildung 4-28: Messergebnisse Einflussfaktor $\triangle S O C$ (links), resultierende Schädigungsfunktionen (rechts)
} 

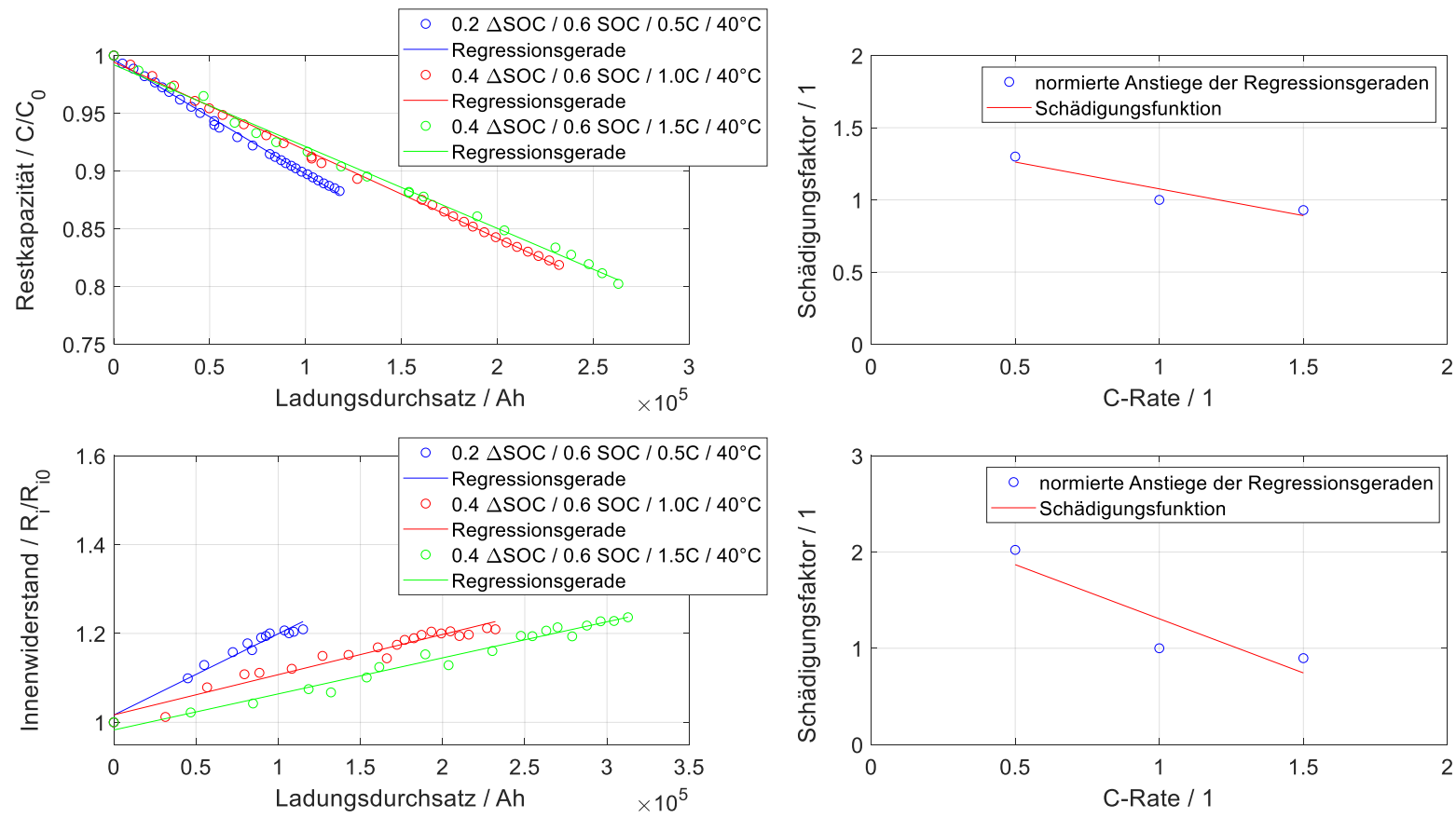

\section{Abbildung 4-29: Messergebnisse Einflussfaktor C-Rate (links), resultierende Schädigungsfunktionen (rechts)}

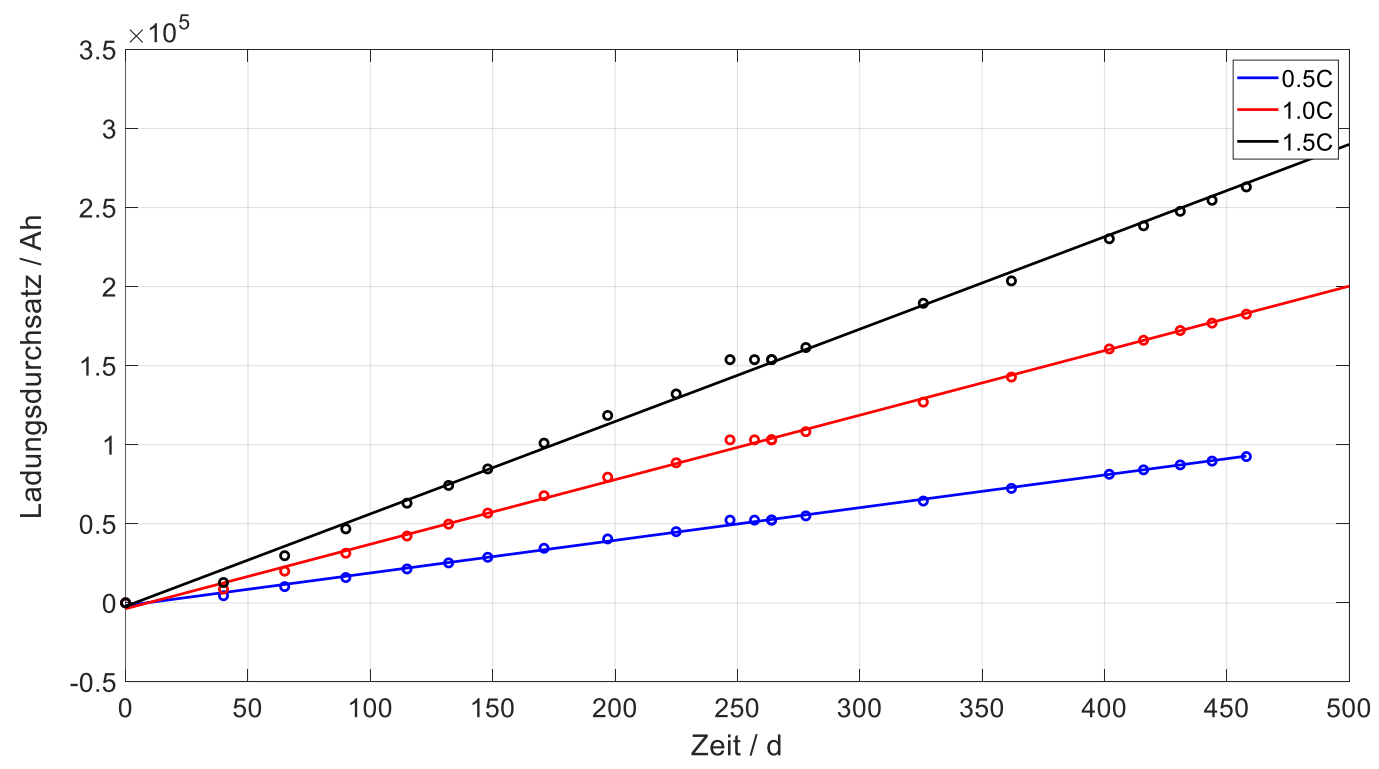

Abbildung 4-30: Ladungsdurchsatz in Abhängigkeit der Versuchsdauer (Messreihe: C-Rate) 
In Abbildung 4-31 sind die Messreihen und die Schädigungsfunktionen des mittleren SOCs der kalendarischen Alterung abgebildet. Sowohl die Kapazität als der Innenwidertand weisen einen parabelförmigen Verlauf auf. Während die Funktion des Widerstands einen sehr guten Fit ermöglicht, enthält die der Kapazität einen unplausiblen Wert an der Stelle SOC =0,6. Dieser liegt höher als der Wert bei $\mathrm{SOC}=0,4$, wobei an dieser Stelle ein niedrigerer Wert erwartet wurde. Aus der Literatur sind ebenfalls parabelförmige Zusammenhänge für die Schädigungswirkung des mittleren SOC bekannt, beispielsweise aus (Schmalstieg et al., 2014). An dieser Stelle werden erneut Messunsicherheiten als Ursache vermutet. Aus den Schädigungsfunktionen geht hervor, dass eine Lagerung der Zellen bei mittlerem Ladezustand die geringste Schädigungswirkung hat.

Der mittlere SOC der zyklischen Alterung kann aufgrund fehlender Messwerte in dieser Arbeit nicht parametriert werden und wird im Alterungsmodell mit konstant eins gesetzt.
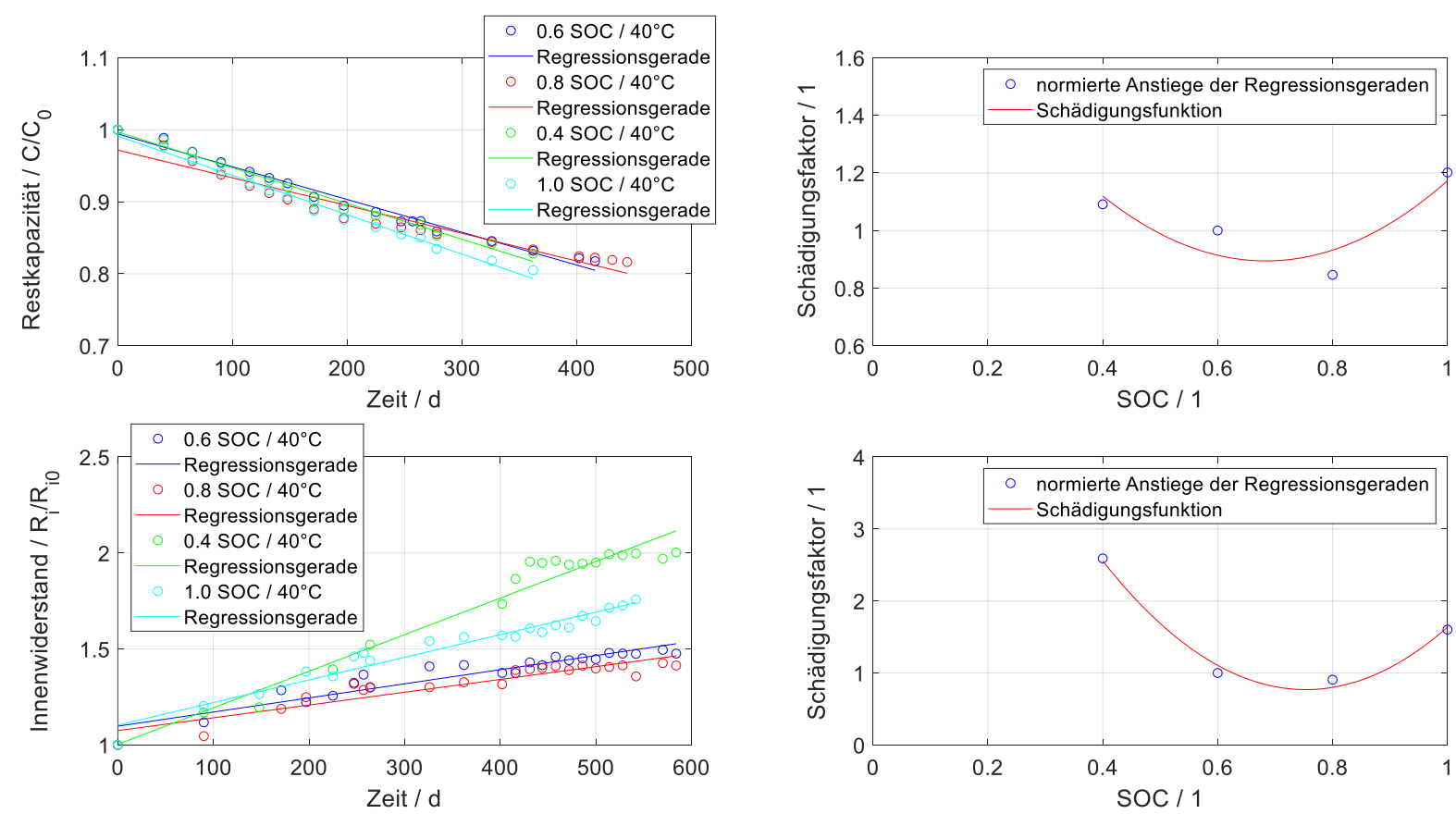

\section{Abbildung 4-31: Messergebnisse Einflussfaktor SOC (kalendarisch) (links), resultierende Schädigungsfunktionen (rechts)}

\section{Zwischenfazit der Messreihenauswertung}

Aus den Ergebnissen der Messwertauswertung können folgende Erkenntnisse für den Betrieb von Elektrobussen zusammengefasst werden: 
- Zwischen $20^{\circ} \mathrm{C}$ und $40^{\circ} \mathrm{C}$ steigt die Schädigung sowohl zyklisch als auch kalendarisch nur moderat an. Unter Berücksichtigung der temperaturabhängigen Leistungsfähigkeit der Batterie (Kapazität, Vgl. Abbildung 4-6 und Strombegrenzung, Vgl. Abbildung 4-9) kann festgehalten werden, dass dies der optimale Betriebsbereich der Batterie ist. Er stellt einen guten Kompromiss aus verfügbarer Leistungsfähigkeit und resultierender Schädigung dar.

- Gemäß der ermittelten Schädigungsfunktion des $\triangle S O C$ s ist eine übermäßig große Ausnutzung des vorhandenen Energieinhalts der Batterie aus Alterungssicht zu vermeiden. Dadurch wird jedoch die Reichweite des Elektrobusses - je nach Wahl des zulässigen SOC-Hubs - teilweise erheblich eingeschränkt. Eine einfache Abhilfemaßnahme stellt die Überdimensionierung der Batterie dar, was aus Kostengründen jedoch vermieden werden soll. Über die Wahl einer geeigneten Ladestrategie kann der $\triangle \mathrm{SOC}$ erheblich reduziert und die Lebensdauer der Batterie verlängert werden ( $\mathrm{Vgl}$. Kapitel 5.3).

- Gemäß der ermittelten Schädigungsfunktion des mittleren SOCs ist es bei einer längeren Abstellung eines Fahrzeugs empfehlenswert, dass sich die Batterie nicht im vollgeladenen oder stark entladenen Zustand befindet. Eine möglichst geringe Alterung wird bei einem mittleren Ladezustand von ca. 50\% erreicht.

\section{Modellbildung}

Es wird auf Basis der Messergebnisse und der ermittelten Schädigungsfunktionen ein empirisches Alterungsmodell erstellt, mit welchem beliebige Arbeitspunkte auch außerhalb der Messmatrix berechnet werden können (Ufert et al., 2018a). Dafür wird die Annahme zugrunde gelegt, dass die einzelnen Schädigungsfunktionen voneinander unabhängig sind, das heißt keine gegenseitigen Einflüsse stattfinden. Der Nachweis zur Gültigkeit dieser Annahme wurde in (Lewerenz, 2018) erbracht. Auf dieser Basis soll folgende Modellgrundlage Anwendung finden:

Beliebige Arbeitspunkte sind über die Multiplikation der jeweiligen Schädigungsfaktoren berechenbar.

Die Funktionsweise dieser Modellgrundlage wird anhand eines einfachen Beispiels in Tabelle 4-11 erläutert. In Blau sind die aus den Messreihen ermittelten Schädigungsfaktoren (gleichzeitig die Stützstellen der Schädigungsfunktionen) für die Einflussfaktoren Temperatur und $\triangle \mathrm{SOC}$ eingetragen. Die übrigen Arbeitspunkte (welche nicht messtechnisch ermittelt wurden) werden nun über Multiplikation der angrenzenden Stützstellen berechnet und sind in Tabelle 4-11 in Grün eingetragen. Auf dieser Basis können nun beliebige Arbeitspunkte unter Berücksichtigung aller vier Einflussfaktoren 
und den dazugehörigen Schädigungsfunktionen ermittelt werden. Im Folgenden wird der Aufbau des empirischen Alterungsmodells detailliert erläutert.

\begin{tabular}{cccc}
\multicolumn{4}{c}{ Tabelle 4-11: Modellgrundlage - Berechnungsbeispiel } \\
\hline & $\mathbf{2 0 ^ { \circ } \mathrm { C }}$ & $\mathbf{4 0}{ }^{\circ} \mathrm{C}$ & $\mathbf{5 0 ^ { \circ } \mathrm { C }}$ \\
\hline $\mathbf{\Delta S O C} \mathbf{2 0} \%$ & 0,61 & $\mathbf{0 , 8 6}$ & 2,41 \\
$\mathbf{\Delta S O C} \mathbf{4 0 \%}$ & $\mathbf{0 , 7 1}$ & $\mathbf{1 , 0 0}$ & $\mathbf{2 , 8 0}$ \\
$\mathbf{\Delta S O C} \mathbf{6 0 \%}$ & 1,14 & $\mathbf{1 , 6 1}$ & 4,51 \\
\hline
\end{tabular}

Aus den Messergebnissen geht hervor, dass sowohl die Kapazitätsabnahme als auch der Anstieg des Innenwiderstands für die kalendarische und die zyklische Alterung linear approximiert werden können. Die mathematische Grundgleichung beider Funktionen lautet somit:

$$
y=m \cdot x+b
$$

Bezogen auf den Anwendungsfall der Alterung ergibt sich folgender Zusammenhang.

$$
\begin{aligned}
& C_{1}=m \cdot Q+C_{0} \\
& R_{\mathrm{i} 1}=m \cdot Q+R_{\mathrm{i} 0}
\end{aligned}
$$

Q Ladungsdurchsatz in Ah der zu betrachtenden Belastung,

$C_{0} \quad$ Kapazität vor der zu betrachtenden Belastung (Ausgangskapazität),

$C_{1}$ reduzierte Kapazität am Ende der betrachteten Belastung,

$R_{\mathrm{i} 0} \quad$ Innenwiderstand vor der zu betrachtenden Belastung,

$R_{\mathrm{i} 1} \quad$ erhöhter Innenwiderstand am Ende der betrachteten Belastung.

Soll statt der zyklischen Alterung die kalendarische Alterung berechnet werden, so ist als unabhängige Größe anstatt des Ladungsdurchsatzes $Q$ die Zeit $t$ in Tagen einzusetzen.

$$
C_{1}=m \cdot t+C_{0}
$$




$$
R_{\mathrm{i} 1}=m \cdot t+R_{\mathrm{i} 0}
$$

Der Anstieg in beiden Funktionen ist definiert als sog. Referenzanstieg. Dieser Anstieg kennzeichnet diejenige Messreihe, welche als Normierungsreihe verwendet wurde. Zur Berechnung beliebiger Parameterkonfigurationen werden die ermittelten Schädigungsfunktionen $S$ hinzugezogen. Über die Multiplikation mit dem jeweiligen Referenzanstieg $m_{\text {ref,zykl }}$ wird die Relation zum Normierungspunkt abgebildet. Daraus resultieren folgende Modellgleichungen:

$$
\begin{gathered}
C_{1}=m_{\mathrm{ref}, \mathrm{zykl}, \mathrm{C}} \cdot S_{\mathrm{Temp}, \mathrm{C}} \cdot S_{\Delta \mathrm{SOC}, \mathrm{C}} \cdot S_{\mathrm{SOC}, \mathrm{C}} \cdot S_{\mathrm{C}-\mathrm{Rate}, \mathrm{C}} \cdot Q+C_{0} \\
R_{\mathrm{i} 1}=m_{\mathrm{ref,zykl,R}} \cdot S_{\mathrm{Temp}, \mathrm{R}} \cdot S_{\Delta \mathrm{SOC}, \mathrm{R}} \cdot S_{\mathrm{SOC}, \mathrm{R}} \cdot S_{\mathrm{C}-\mathrm{Rate}, \mathrm{R}} \cdot Q+R_{\mathrm{i} 0}
\end{gathered}
$$

Es gilt zu beachten, dass für Kapazität und Innenwiderstand unterschiedliche Referenzanstiege gelten.

$$
m_{\text {ref,zykl,C }} \neq m_{\text {ref,zykl, } \mathrm{R}_{\mathrm{i}}}
$$

Für die Bestimmung der kalendarischen Alterung sind lediglich die Schädigungsfaktoren für die Temperatur und den mittleren SOC sowie der jeweilige Referenzanstieg für Kapazität und Innenwiderstand zu verwenden. Der Zusammenhang aus Formel (4-33) gilt dafür in analoger Weise.

$$
\begin{gathered}
C_{1}=m_{\mathrm{ref}, \mathrm{kal}, \mathrm{C}} \cdot S_{\mathrm{Temp}, \mathrm{C}} \cdot S_{\mathrm{SOC}, \mathrm{C}} \cdot t+C_{0} \\
R_{\mathrm{i} 1}=m_{\mathrm{ref,kal,R}, \mathrm{i}} \cdot S_{\mathrm{Temp}, \mathrm{R}} \cdot S_{\mathrm{SOC}, \mathrm{R}} \cdot t+R_{\mathrm{i} 0}
\end{gathered}
$$

\section{Workflow zur Berechnung realer Stromprofile}

Die empirischen Modellgleichungen ((4-31), (4-32), (4-34) und (4-35)) wurden auf Basis der Messergebnisse der Testmatrix entwickelt, welche wiederum auf der kontinuierlichen Zyklisierung der Zellen mittels konstanter Rechteck-Stromprofile bei nahezu konstanten Umgebungstemperaturen basieren. Soll die Alterung nun für eine reale Anwendung wie einen Elektrobus ermittelt werden, so ist die Berechnung eines dynamischen Stromprofils mit unterschiedlichen C-Raten, $\triangle \mathrm{SOCs}$ und Temperaturen erforderlich. Eine Anwendung der Modellgleichungen scheint auf den ersten Blick nur schwer möglich. Dafür sind konstante Belastungsbedingungen erforderlich, welche im Betrieb des Elektrobusses in dieser Form nicht auftreten. Es wird im Folgenden ein 
Workflow entwickelt, welcher eine Bewertung dynamischer Stromprofile anhand der empirischen Modellgleichungen ermöglicht.

Aus Abbildung 3-1 geht hervor, dass für die Batteriesimulation zwei verschiedene Zeitebenen gewählt wurden. In der Mikrozeitschrittebene wird die elektrothermische Belastung der Batterie ermittelt. In der Makrozeitschrittebene soll nun die resultierende Alterung berechnet werden. Ein Fahrszenario wird demnach zunächst elektrothermisch berechnet und die Ergebnisse anschließend der Alterungsberechnung zugeführt. Dabei muss es sich um ein abgeschlossenes Fahrszenario handeln, welches dadurch gekennzeichnet ist, dass der Start-SOC und der End-SOC gleich sind. Im Anwendungsfall Elektrobus ist dies beispielsweise ein Betriebstag mit anschließender Ladung im Depot.

Aus der Mechanik ist die Wöhlerkennlinie zur Beurteilung der Betriebsfestigkeit mechanischer Bauteile bekannt. Die Kennlinie gibt für konkrete Spannungsamplituden die Anzahl maximal ertragbarer Schwingspiele bis zum Bruch des Bauteils an. In realen Anwendungen sind die Bauteile jedoch nicht nur einer konkreten konstanten Belastung ausgesetzt. Vielmehr ist ein reales Belastungsszenario eine Zusammensetzung unterschiedlicher Teilbelastungen (sog. Belastungskollektive). Um dennoch die Wöhlerkennlinie zur Festigkeitsbeurteilung heranziehen zu können, werden die Belastungskollektive in einzelne Teilkollektive zerlegt, bei welchen dann konstante Bedingungen (also Spannungsamplituden) unterstellt werden. Für diese kann mittels der Wöhlerkennlinie die anteilige Schädigung ermittelt werden. Für die Ermittlung der Gesamtschädigung wird nun die Methode der linearen Schadensakkumulation angewendet. Diese besagt, dass die Einzelschädigungen zur Gesamtschädigung aufaddiert werden können. Die zeitliche Reihenfolge der Belastung geht dabei verloren. Es gilt die Annahme, dass diese keinen Einfluss auf das Ergebnis hat.

Die Methode der linearen Schadensakkumulation wird auch auf das empirische Batteriealterungsmodell angewendet. Zur Zerlegung eines realen Fahrszenarios (Lastzyklus) in einzelne Teilzyklen (analog zur Zerlegung eines Belastungskollektivs in Teilkollektive) wird das Rainflow-Zählverfahren angewendet. Darin werden sog. Schädigungs-Fundamentalereignisse definiert, welche jeweils eine geschlossene Hysterese darstellen. Die Hystereseschleifen werden durch eine Klassierung von Umkehrpunkten des zeitlichen Belastungsverlaufs ermittelt. Auf den Anwendungsfall der Batteriealterung übertragen bedeutet dies, dass der zeitliche Verlauf der Belastung in geschlossene Teilzyklen unterteilt werden muss. Ein solcher geschlossener Teilzyklus ist dann gegeben, wenn Start-SOC und End-SOC gleich sind. Demnach ist der SOC-Verlauf die maßgebliche Größe zur Zyklusanalyse. Zusätzlich werden zur Charakterisierung der Teilzyklen die Temperatur und der Stromverlauf benötigt, da beide Größen Einflussfaktoren auf die Batteriealterung sind. Die Funktionsweise des Rainflow-Zählverfahrens sowie die Anwendung auf die Zyklusanalyse der Batteriebelastung sind in (Farhat, 2018) ausführlich beschrieben. Der Workflow zur Berechnung der resultierenden Alterung durch ein reales Fahrszenario ist in Abbildung 4-32 dargestellt. 


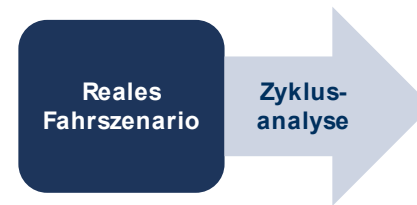

\section{Abbildung 4-32: Workflow zur Berechnung dynamischer Stromprofile}

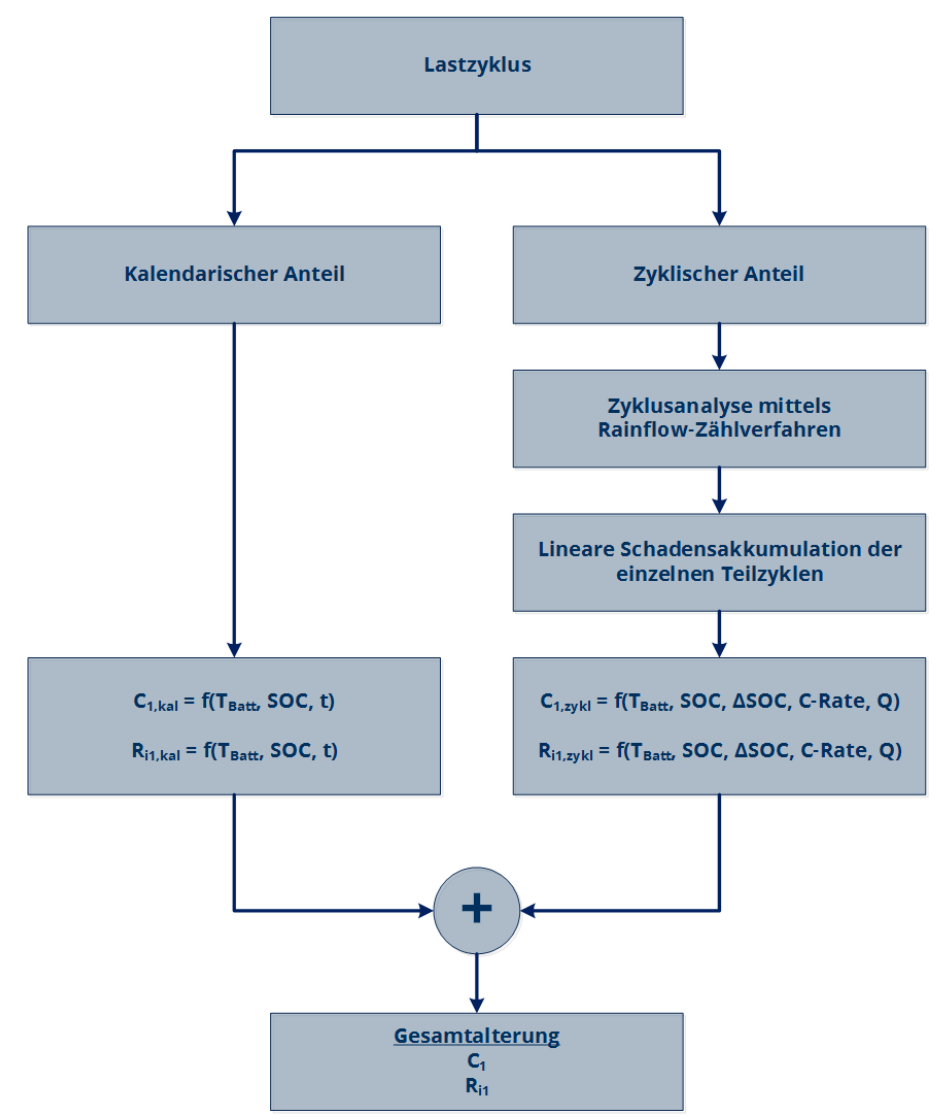

Abbildung 4-33: Superposition von kalendarischer und zyklischer Alterung

Das bisher beschriebene Vorgehen bezieht sich nur auf die Beurteilung eines Fahrszenarios, also der zyklischen Alterung. Ein vollständiger Lastzyklus einer Batterie kann jedoch auch Anteile enthalten, in denen das Fahrzeug nicht bewegt und die Batterie nicht belastet wird. In diesem Fall muss zur Berechnung der Gesamtalterung neben dem zyklischen auch der kalendarische Anteil betrachtet werden. Dafür wird ein vollständiger Lastzyklus zunächst in die Anteile zyklisch und kalendarisch zerlegt. Gemäß der Definition der linearen Schadensakkumulation spielt die Reihenfolge der Belastung keine Rolle. Für die kalendarische Alterung kann die resultierende Schädigung unmittelbar unter Anwendung der empirischen Modellgleichungen berechnet werden. Zur Berechnung der zyklischen Alterung wird der Workflow gemäß Abbildung 4-32 
angewendet. Die Gesamtalterung eines vollständigen Lastzyklus wird nun mittels Superposition aus kalendarischer und zyklischer Alterung berechnet. Das Vorgehen ist in Abbildung 4-33 veranschaulicht.

\section{Validierung des Alterungsmodells}

Im Folgenden werden das entwickelte Alterungsmodell und der Workflow zur Berechnung realer Fahrszenarien anhand zweier Labormessungen validiert. Dafür wurden zwei Zellen der gleichen Charge wie die Zellen zur Modellbildung und Parametrierung mit dynamischen Stromprofilen belastet. Die Zelltemperatur konnte innerhalb enger Grenzen bei nahezu $50^{\circ} \mathrm{C}$ konstant gehalten werden. Die Fahrszenarien sind im Folgenden als Zyklus A und Zyklus B bezeichnet und in Abbildung 4-34 und Abbildung 4-35 dargestellt. Die Zyklisierung beider Zellen wurde analog zum Vorgehen der anderen Messreihen in regelmäßigen Abständen unterbrochen, um den bis dahin vorangeschrittenen Alterungsprozess messtechnisch zu erfassen.

Analog zu den Zellen der Parametrierung wurde bei beiden Zyklen jeweils das Kapazitäts-EOL-Kriterium (80\% der Nennkapazität) erreicht. Der Anstieg des Innenwiderstands erreichte jeweils nur Werte kleiner 150\% (bezogen auf den Nennwert). Daraus kann geschlussfolgert werden, dass die im Rahmen dieser Arbeit vermessenen Zellen des Elektrobusses der Linie 79 in Dresden vermutlich aufgrund einer zu geringen Kapazität zu ersetzen sind, nicht jedoch aufgrund eines zu hohen Innenwiderstands. Beide Zyklen werden mittels des Alterungsmodells simuliert, bis die durchgesetzte Ladungsmenge der der vermessenen Zellen entspricht. Im Folgenden werden die Mess- und Simulationsergebnisse beider Zyklen vergleichend dargestellt und ausgewertet.
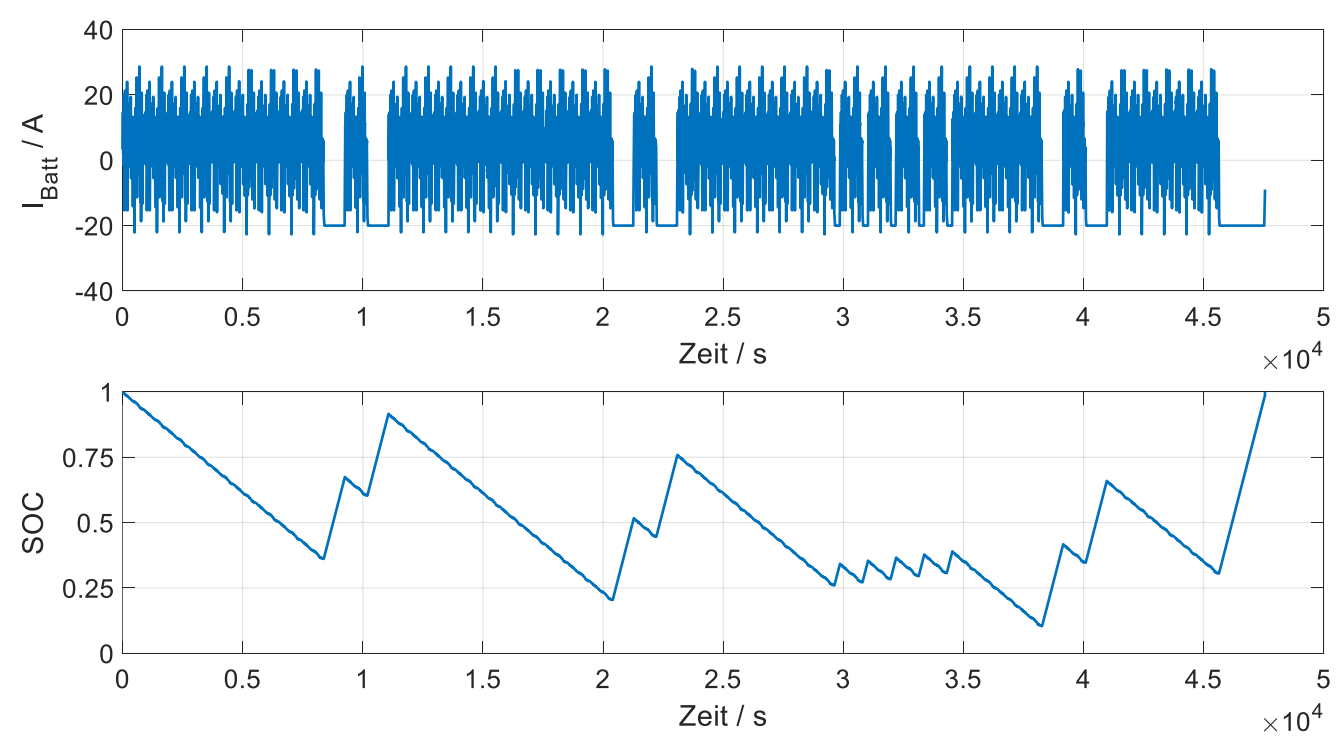

Abbildung 4-34: Zyklus A für die Validierung des Alterungsmodells 

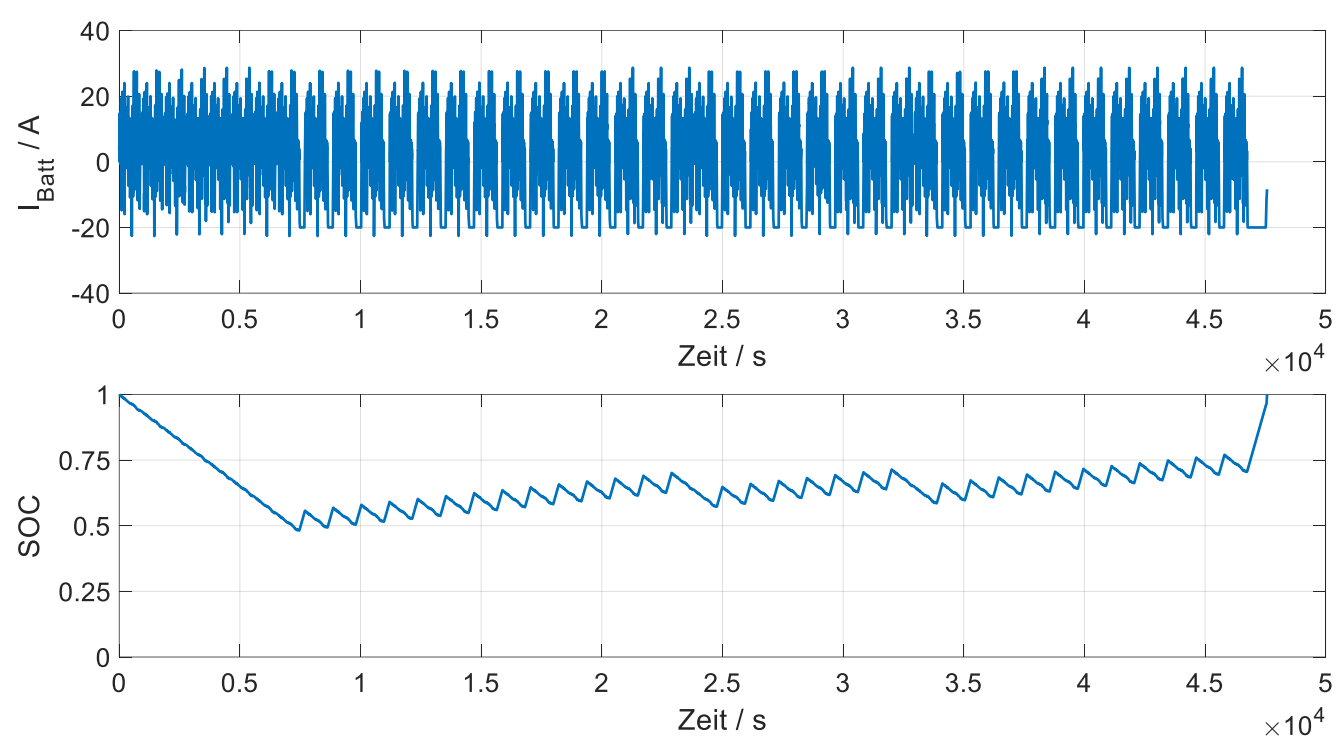

Abbildung 4-35: Zyklus B für die Validierung des Alterungsmodells

\section{Zyklus A}

In Abbildung 4-36 sind die simulierten und gemessenen Verläufe für die Abnahme der Kapazität und den Anstieg des Innenwiderstands für Zyklus A vergleichend dargestellt. Es ist erkennbar, dass für die Abnahme der Kapazität ein Ladungsdurchsatz von ca. 38.500 Ah erreicht wurde, während der letzte verfügbare Messpunkt zur Bestimmung des Innenwiderstandsanstiegs bei ca. 30.900 Ah liegt. Für die Kapazität und den Innenwiderstand wurden jeweils Regressionsgeraden auf Basis der Messpunkte ermittelt. Die Ergebnisse der Messung und der Simulation sind in Tabelle 4-12 zusammengefasst. Daraus geht hervor, dass die Abnahme der Kapazität in sehr geringem Maße unterschätzt, der Anstieg des Innenwiderstands dagegen überschätzt wird (Vgl. Abbildung 4-36). In Tabelle 4-12 ist zusätzlich der jeweilige relative Modellfehler angegeben, welcher sich aus dem Verhältnis von Messung und Simulation berechnet.

Tabelle 4-12: Vergleich Messung und Simulation Zyklus A

\begin{tabular}{cc}
\hline Kapazität & Innenwiderstand \\
\hline$\frac{C_{\text {mess }}}{C_{0}}(Q=38.500 A h)=0,792$ & $\frac{R_{\mathrm{i}, \text { mess }}}{R_{\mathrm{io}}}(Q=30.900 \mathrm{Ah})=1,299$ \\
$\frac{C_{\text {sim }}}{C_{0}}(Q=38.500 A h)=0,796$ & $\frac{R_{\mathrm{i}, \text { sim }}}{R_{\mathrm{io}}}(Q=30.900 \mathrm{Ah})=1,341$ \\
$\left|1-\frac{C_{\text {sim }}}{C_{\text {mess }}}\right|=0,51 \%$ & $\left|1-\frac{R_{\mathrm{i}, \text { sim }}}{R_{\mathrm{i}, \text { mess }}}\right|=3,23 \%$ \\
\hline
\end{tabular}



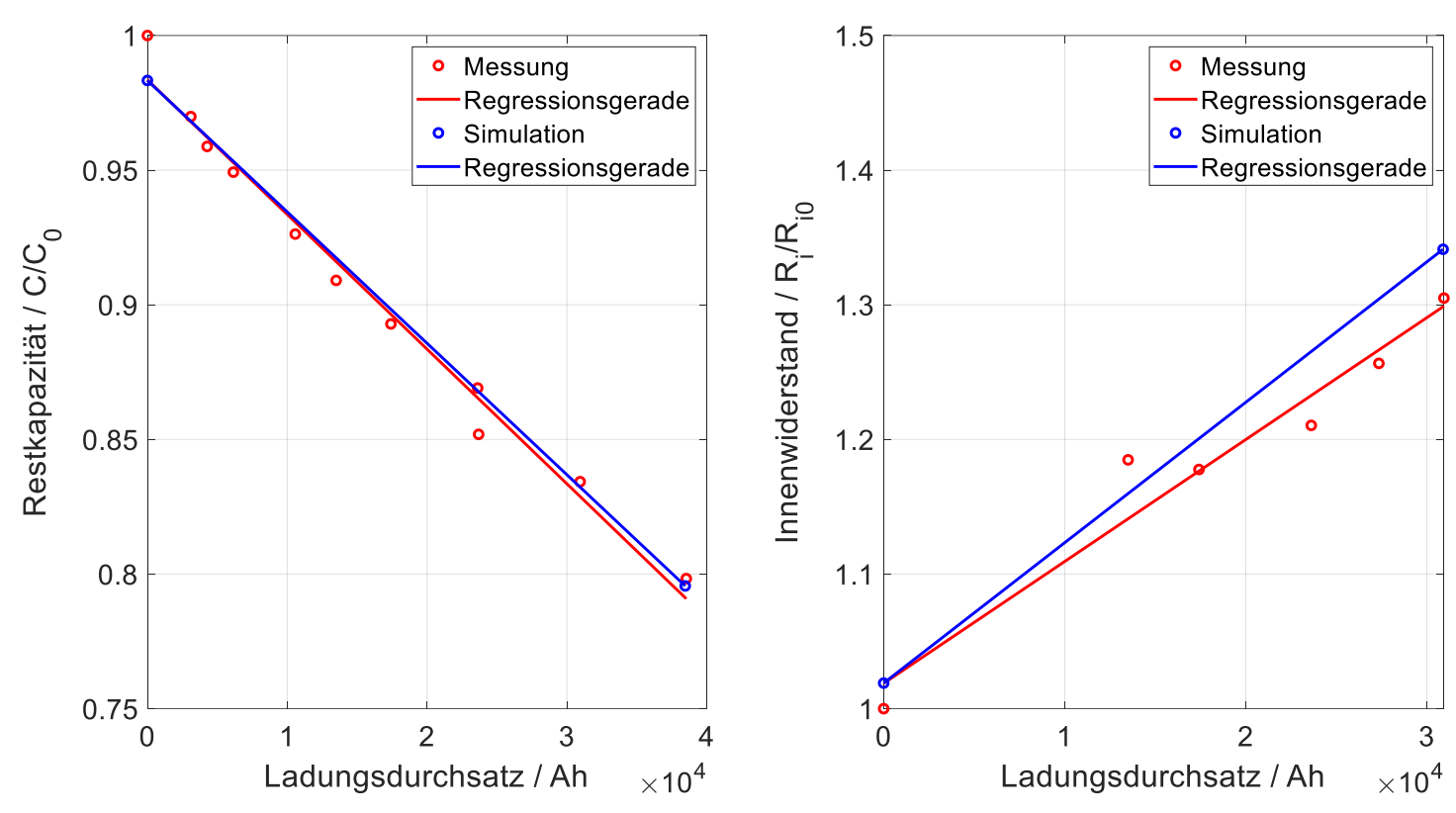

Abbildung 4-36: Validierungsergebnisse Zyklus A

\section{Zyklus B}

In Abbildung 4-37 sind die simulierten und gemessenen Verläufe für die Abnahme der Kapazität und den Anstieg des Innenwiderstands für Zyklus B vergleichend dargestellt. Im Gegensatz zu Zyklus A wurde hier sowohl für die Abnahme der Kapazität als auch für den Anstieg des Innenwiderstands ein Ladungsdurchsatz von ca. 38.500 Ah erreicht. Die Ergebnisse der Messung und der Simulation sind in Tabelle 4-13 zusammengefasst. Daraus geht hervor, dass sowohl die Abnahme der Kapazität als auch der Anstieg des Innenwiderstands unterschätzt werden. In Tabelle 4-13 ist zusätzlich der jeweilige relative Modellfehler angegeben, welcher sich aus dem Verhältnis von Messung und Simulation berechnet.

Tabelle 4-13: Vergleich Messung und Simulation Zyklus B

\begin{tabular}{cl}
\hline Kapazität & Innenwiderstand \\
\hline$\frac{C_{\text {mess }}}{C_{0}}(Q=38.500 A h)=0,804$ & $\frac{R_{\mathrm{i}, \mathrm{mess}}}{R_{\mathrm{io}}}(Q=38.500 \mathrm{Ah})=1,375$ \\
$\frac{C_{\text {sim }}}{C_{0}}(Q=38.500 \mathrm{~A} h)=0,874$ & $\frac{R_{\mathrm{i}, \mathrm{sim}}}{R_{\mathrm{io}}}(Q=38.500 \mathrm{Ah})=1,334$ \\
$\left|1-\frac{C_{\text {sim }}}{C_{\text {mess }}}\right|=8,71 \%$ & $\left|1-\frac{R_{\mathrm{i}, \text { sim }}}{R_{\mathrm{i}, \mathrm{mess}}}\right|=2,98 \%$ \\
\hline
\end{tabular}



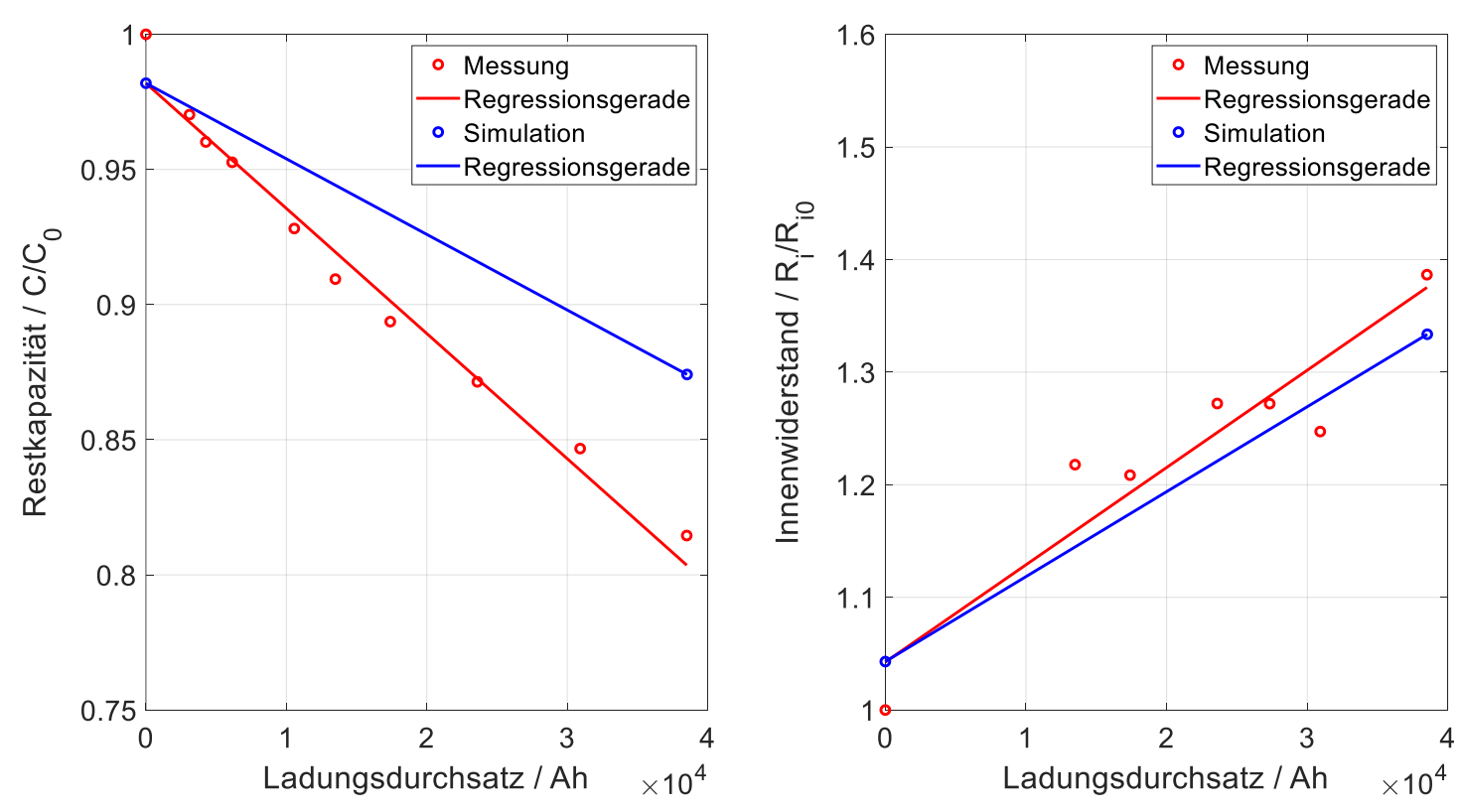

Abbildung 4-37: Validierungsergebnisse Zyklus B

\section{Fazit der Validierung}

Die Validierungsergebnisse sind für beide Zyklen unterschiedlich zu bewerten. Zyklus A weist mit 0,51\% nur einen sehr kleinen Modellfehler bei der Kapazität auf. Der Innenwiderstand hat mit 3,23\% einen größeren, jedoch immer noch akzeptablen Fehler. Bei Zyklus B ist dieser mit 8,71\% bei der Kapazität sehr hoch, während der Innenwiderstand mit 2,98\% im Bereich des Zyklus A liegt.

Zur Einordnung der Ergebnisse sind die Charakteristika der SOC-Verläufe beider Zyklen zu beachten. Der SOC-Verlauf von Zyklus A beinhaltet eine breite Variation einzelner Teilzyklen, welche durch kleine, mittlere und große SOC-Hübe gekennzeichnet sind. Zyklus B hingegen ist von vielen kleinen und vergleichsweise gleichmäßigen SOCHüben (auch als Mikrozyklen bezeichnet) geprägt. Grundsätzlich entspricht Zyklus A einer typischen Stadtbusanwendung, während Zyklus B einen eher synthetischen Charakter hat und in realen Anwendungen nur selten vorkommen sollte. Das Validierungsergebnis von Zyklus A zeigt, dass eine typische Belastung, wie diese bei Elektrobussen im städtischen ÖPNV auftritt, mit sehr guter Genauigkeit bewertet werden können. Dabei ist das Ergebnis des Kapazitätsverlaufs höher zu bewerten als das des Innenwiderstands, da in allen Messungen stets das Kapazitäts-EOL-Kriterium zuerst erreicht wurde. Die Zelle des Zyklus B ist hinsichtlich der Kapazität hingegen deutlich schneller gealtert als vom Modell berechnet wurde. Offensichtlich treten bei Belastungen mit einer hohen Anzahl an Mikrozyklen Alterungseffekte auf, welche vom Modell nicht ausreichend abgebildet werden. Zusammenfassend kann festgehalten 
werden, dass das Alterungsmodell eine gute Genauigkeit zur Berechnung typischer Elektrobuszyklen aufweist.

\section{Grenzen und Einschränkungen des Modells}

Das Alterungsmodell wurde mit dem Ziel entwickelt, die Schädigung der Traktionsbatterien bis zu den definierten Abbruchkriterien von 80\% Restkapazität bzw. 200\% Innenwiderstand zu ermitteln und zu beurteilen. Nach Erreichen dieser Grenzen sind die Batterien jedoch nicht physisch defekt, sondern genügen lediglich nicht mehr den Anforderungen eines Elektrobusses. In (Schuster et al., 2015) wurde das Alterungsverhalten von Li-Ionen Zellen von $80 \%$ Restkapazität abwärts untersucht. Nahezu alle vermessenen Zellen weisen dabei (unabhängig von der konkreten Belastung) einen Übergang vom linearen Alterungsverhalten zu einem nichtlinearen Verhalten auf. Dieser Zusammenhang wurde im Rahmen dieser Arbeit nicht untersucht, da der Bereich für den gewählten Betrachtungsraum keine Relevanz hat. Für das entwickelte Alterungsmodell kann demnach festgehalten werden, dass die Gültigkeit nur innerhalb der definierten Grenzen von 80\% Restkapazität bzw. 200\% Innenwiderstand besteht.

Des Weiteren sei angemerkt, dass aufgrund des sehr kleinen Untersuchungsraums keine statistische Absicherung des Modells möglich ist. Aus der Messmatrix (Vgl. Tabelle 4-9 und Tabelle 4-10) geht hervor, dass jeder Arbeitspunkt genau einmal vermessen wurde. Damit können ggf. Ausreißer nicht identifiziert werden. Dafür wäre eine Vervielfachung der Messmatrix notwendig gewesen, was aus Ressourcengründen nicht möglich war. Zudem wurde beispielsweise in (Baumhöfer et al., 2014) und (Paul, 2015) gezeigt, dass selbst gleichartige Zellen der gleichen Charge bei identischer Belastung produktionsbedingte Streuungen der Kapazität und des Innenwiderstands aufweisen, welche die Geschwindigkeit der Alterung beeinflussen können.

\subsection{Modell der Nachladepunkte}

Für die Ausgestaltung der streckenseitigen Ladepunkte existiert eine Vielzahl unterschiedlicher Varianten, welche in Kapitel 2.1 beschrieben wurden. Unabhängig von der Ladetechnologie sowie der elektrischen und mechanischen Auslegung müssen alle Varianten der Anforderung genügen, die geforderte Ladeleistung sicher und zuverlässig in das Fahrzeug zu übertragen. Für die Systemsimulation soll ein generischer Ansatz entwickelt werden, mit welchem die unterschiedlichen Technologien und Konzepte parametrierbar sind. Dafür ist es notwendig, die in einem Ladepunkt verbauten elektrischen Komponenten zu analysieren. In Abbildung 4-38 sind die elektrotechnischen Komponenten eines stromgesteuerten, konduktiven Ladepunkts sowie der Leistungsfluss in das Fahrzeug dargestellt. Darin ist erkennbar, dass ein Ladepunkt aus energetischer Sicht hauptsächlich aus folgenden Komponenten besteht: 
- leistungselektronische Wandler zur Spannungs- bzw. Stromsteuerung,

- Transformator zur Potentialtrennung und Spannungsanpassung.

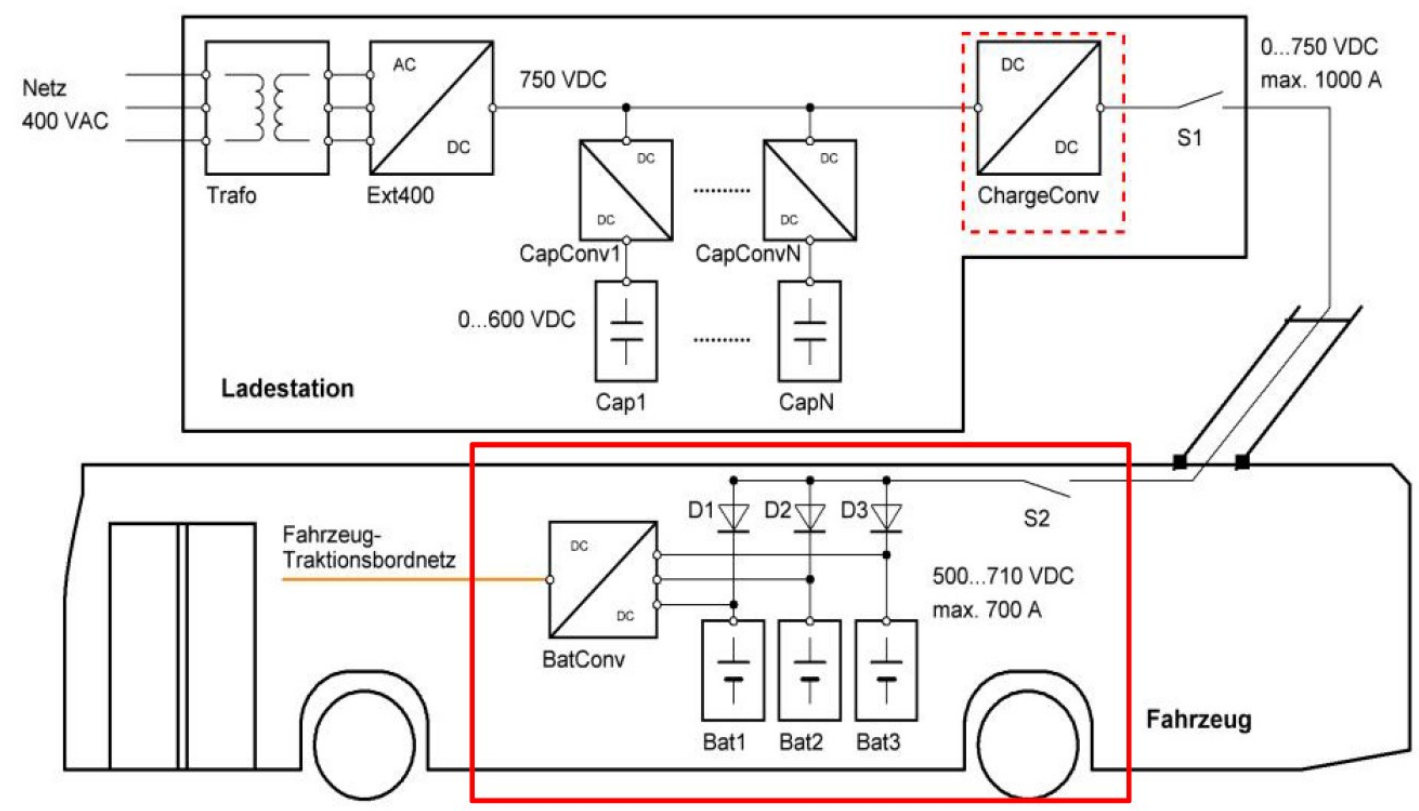

Abbildung 4-38: Übersicht eines konduktiven Ladepunkts (Büchner, 2015)

In Abbildung 4-38 sind zusätzlich zwei Superkondensatoren zur Pufferung von Leistungsspitzen integriert, welche jedoch optional sind.

In dieser Arbeit wird als Randbedingung angenommen, dass die vom Fahrzeug geforderte Ladeleistung zu jedem Zeitpunkt vollumfänglich bereitgestellt werden kann. Eine Rückwirkung auf das speisende Energienetz und daraus resultierende Leistungsbegrenzungen sind nicht Gegenstand der Betrachtungen.

Die elektrotechnischen Komponenten des Ladepunkts sind aus energetischer Sicht wesentlich durch ihren jeweiligen Wirkungsgrad definiert. Aus Abbildung 4-38 geht hervor, dass diese ausschließlich in Reihe geschaltet sind. Dies gilt für induktive Ladepunkte analog. Die Puffereinrichtungen werden dabei nicht betrachtet. Der Wirkungsgrad des Ladepunkts $\eta_{\text {Ladepunkt }}$ berechnet sich wie folgt:

$$
\eta_{\text {Ladepunkt }}=\eta_{\text {Trafo }} \cdot \eta_{\text {Wandler1 }} \cdot \eta_{\text {Wandler2 }}
$$

In der Systemsimulation wird die Ladeleistung $P_{\text {Ladung }}$ am Fahrzeug fest definiert. Für die spätere Berechnung der Kosten des Ladepunkts (Vgl. Kapitel 4.3) wird die zu 
installierende Leistung am Ladepunkt $P_{\text {Ladepunkt }}$ mittels des Wirkungsgrades wie folgt berechnet.

$$
P_{\text {Ladepunkt }}=\frac{P_{\text {Ladung }}}{\eta_{\text {Ladepunkt }}}
$$

Über den einfachen Ansatz einer Wirkungsgradkette können die Ladetechnologien konduktiv und induktiv gleichermaßen abgedeckt werden. Systembedingt ergeben sich bei konduktiver Übertragung leicht bessere Gesamtwirkungsgrade als bei induktiver Übertragung. In (BMVI, 2016) sind für beide Ladetechnologien durchschnittliche Wirkungsgrade angegeben, welche in realen Anwendungen messtechnisch ermittelt wurden (Vgl. Tabelle 4-14).

Tabelle 4-14: Wirkungsgrade verschiedener Ladetechnologien

(BMVI, 2016)

\begin{tabular}{llc}
\hline Technologie & Ort & Wirkungsgrad \\
\hline Induktiv & Braunschweig & $90,5 \%$ \\
& Berlin & $90,2 \%$ \\
& Mannheim & $91,0 \%$ \\
Konduktiv & Dresden (Gruna) & $94,7 \%$ \\
& Dresden (Dreyßigplatz) & $93,0 \%$ \\
\hline
\end{tabular}

Die am Ladepunkt zu installierende Leistung $P_{\text {Ladepunkt }}$ ist gleichzeitig auch die geforderte Anschlussleistung am Netzanschlusspunkt des speisenden Energienetzes. Die Anbindung an dieses ist meist derart kurz, dass die darin anfallenden Verluste in guter Näherung vernachlässigt werden können. In besonderen Fällen, in denen eine sehr lange Zuleitung vom Netzanschlusspunkt an den Ladepunkt realisiert werden muss, ist eine gesonderte Betrachtung der darin anfallenden Verlustleistung erforderlich.

\subsection{Kostenmodell}

In Kapitel 3.4 wurde die Minimierung der Systemkosten als Ziel der mehrdimensionalen Optimierung definiert. Dafür wird in diesem Abschnitt ein Kostenmodell entwickelt. Dabei handelt es sich um eine Differenzkostenbetrachtung mit dem Ziel der Unterscheidbarkeit verschiedener Auslegungsvarianten einer Elektrobuslinie. Die einzelnen Bestandteile des Modells sind in Abbildung 4-39 dargestellt. 


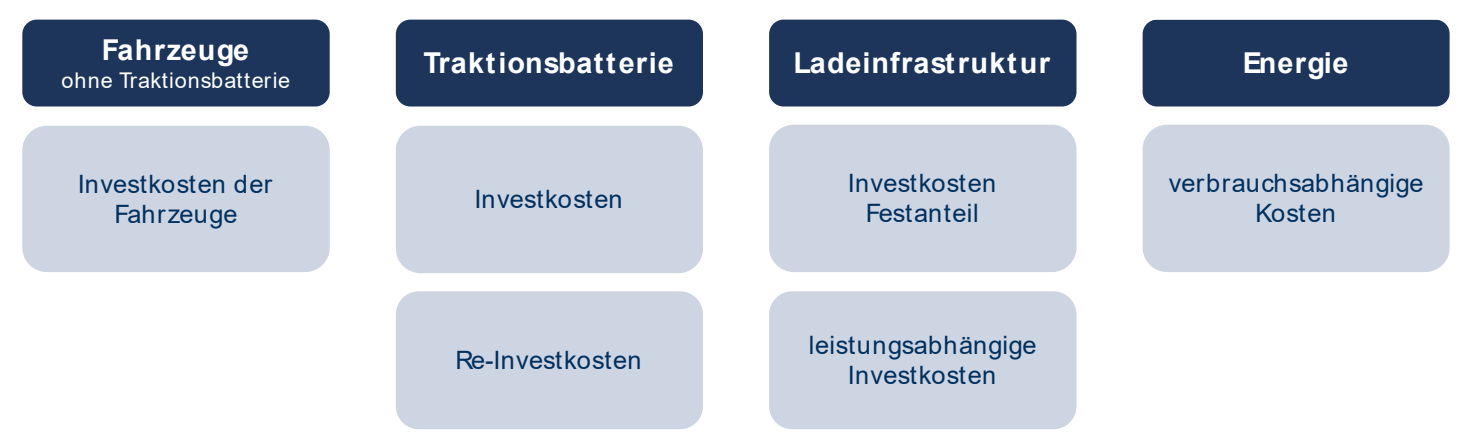

Abbildung 4-39: Bestandteile des Kostenmodells

Zu den weiteren Kostenbestandteilen, welche nicht in Abbildung 4-39 aufgelistet sind, zählen beispielsweise die Ladeinfrastruktur im Depot, spezielle Werkstattausrüstungen für Elektrobusse oder das Fahrpersonal. Im Rahmen der Arbeit wird davon ausgegangen, dass diese Kostenbestandteile bei allen betrachteten Konfigurationen innerhalb der Parametervariation zu jeweils gleichen Anteilen vorhanden sind. Demnach tragen sie nicht zu einer Unterscheidbarkeit der Konfigurationen bei, sondern stellen vielmehr einen konstanten Offset dar und sind deshalb nicht Teil der Differenzkostenbetrachtung. Im Folgenden werden die einzelnen Kostenbestandteile nach Abbildung 4-39 detailliert erläutert.

\section{Fahrzeuge (ohne Traktionsbatterie)}

Die Anzahl der Fahrzeuge $n_{\mathrm{Fzg}}$ hat eine hohe betriebliche Relevanz und berechnet sich aus der Umlaufzeit $t_{\text {Umlauf }}$ und der zu realisierenden Taktzeit $t_{\text {Takt }}$.

$$
n_{\mathrm{Fzg}}=\frac{t_{\text {Umlauf }}}{t_{\text {Takt }}}
$$

Demnach würden für einen 120-minütigen Umlauf im 10 min Takt 12 Fahrzeuge benötigt. Der Kostenbestandteil der Fahrzeuge beinhaltet nicht die notwendige Hochvolt-Traktionsbatterie. Ein üblicher Lebenszyklus eines Dieselbusses beträgt zwischen 10 und 15 Jahren. Für Elektrobusse gilt als Mindestanforderung, dass ein solcher Lebenszyklus ebenfalls erreicht wird. In (Knote et al., 2017) wird den Berechnungen beispielsweise eine Fahrzeuglebensdauer von 14 Jahren zugrunde gelegt, in (Bloomberg, 2018) wird diese mit 15 Jahren definiert. Dagegen wird in (Berthold, 2019) ein Abschreibungszeitraum von 20 Jahren angenommen. Es wird im Rahmen der Arbeit davon ausgegangen, dass die betrachteten Elektrobusse innerhalb des Zeitraums nicht ersetzt werden müssen. Dies gilt jedoch nicht für die Traktionsbatterien, weshalb diese gemäß Abbildung 4-39 gesondert betrachtet werden. 
In Kapitel 3.1 wurde als Randbedingung definiert, dass der Betrieb keine Variationsgröße ist und damit konstant bleibt. Somit wird eine Änderung der Umlaufzeit und der Taktzeit ausgeschlossen. Die Anzahl der benötigten Fahrzeuge $n_{\text {Fzg }}$ ist für jede technisch realisierbare Konfiguration innerhalb einer Optimierungsrechnung gleich. Dennoch wird diese für die Berechnung der Kostenanteile Traktionsbatterie und Energieverbrauch benötigt. Die Gesamtkosten für die Fahrzeuge $K_{\text {Fzg,ges }}$ berechnen sich auf Basis der Einzelkosten je Fahrzeug $K_{\text {Fzg }}$.

$$
K_{\mathrm{Fzg}, \mathrm{ges}}=K_{\mathrm{Fzg}} \cdot n_{\mathrm{Fzg}}
$$

\section{Traktionsbatterie}

Die Traktionsbatterie ist zum aktuellen Zeitpunkt die teuerste Einzelkomponente eines batterie-elektrischen Fahrzeugs (Kampker et al., 2018), was für Elektrobusse in analoger Weise gilt. Des Weiteren ist die Batterie, wie in Kapitel 4.1.5 beschrieben, einem ständigen Alterungsprozess ausgesetzt. Die Labormessungen und Simulationen mittels des entwickelten Alterungsmodells zeigen, dass in den meisten Anwendungsfällen ein Tausch bzw. Ersatz der Batterie innerhalb des Fahrzeuglebenszyklus (ca. 10 - 15 Jahre) erforderlich sein wird.

Dieser Kostenbestandteil ist zweistufig aufgebaut. Es werden sowohl die anfänglichen Kosten als auch die nachgelagerten Kosten, hervorgerufen durch den alterungsbedingten Austausch der Batterie, betrachtet. Zunächst erfolgt die Kostenberechnung für die Anzahl der Fahrzeuge $n_{\text {Fzg }}$ mit der jeweils ersten Batterie. Dabei ist der Energieinhalt $E_{\text {Batt }}$ die entscheidende Beurteilungsgröße. In dieser Arbeit wird ein linearer Zusammenhang zwischen Energieinhalt der Batterie und den Kosten angenommen. Die Gesamtkosten der ersten Traktionsbatterien aller Fahrzeuge $K_{\text {Batt,ges,Invest }}$ berechnen sich mittels der spezifischen Kosten $k_{\text {Batt }}(E U R / k W h)$.

$$
K_{\mathrm{Batt}, \mathrm{ges}, \text { Invest }}=K_{\mathrm{Batt}, \mathrm{Fzg}, \text { Invest }} \cdot n_{\mathrm{Fzg}}=k_{\mathrm{Batt}} \cdot E_{\mathrm{Batt}} \cdot n_{\mathrm{Fzg}}
$$

Für die Berechnung der gesamten Batteriekosten über der betrachteten Fahrzeuglebensdauer $t_{\mathrm{LC}, \mathrm{Fzg}}$ wird die Anzahl der dafür notwendigen Batterien $n_{\mathrm{Batt} \text {,ges }}$ benötigt. Die berechnet sich gemäß Formel (4-41) unter Berücksichtigung der simulierten Batterielebensdauer $t_{\mathrm{EOL}, \mathrm{Batt}}$.

$$
n_{\mathrm{Batt}, \mathrm{ges}}=\frac{t_{\mathrm{LC}, \mathrm{Fzg}}}{t_{\mathrm{EOL}, \mathrm{Batt}}}
$$

Die Gesamtkosten der Traktionsbatterien $K_{\text {Batt,ges }}$ (Invest und Re-Invest) berechnen sich wie folgt: 


$$
K_{\text {Batt,ges }}=K_{\text {Batt,ges,Invest }} \cdot n_{\text {Batt,ges }}
$$

In der Simulationsrechnung werden nichtganzzahlige Werte für die Anzahl der Batterien berücksichtigt. Damit kann eine Verzerrung der Ergebnisse aufgrund von überproportionalen Rundungen ausgeschlossen werden.

\section{Ladeinfrastruktur}

Der Kostenanteil der Ladeinfrastruktur ist zweistufig aufgebaut, um dem Aufbau einer Ladesäule Rechnung zu tragen. Dabei sind nicht nur die elektrotechnische Ausrüstung zu beachten, sondern auch die notwendigen Aufwendungen für Hoch- und Tiefbau sowie den Anschluss an das speisende Energienetz. Der erstgenannte Anteil ist leistungsabhängig und wird als lineare Funktion in Abhängigkeit der zu installierenden Leistung ausgeführt. Dieser Anteil ist für alle Ladepunkte des Systems gleich. Der zweite Anteil wird als Festanteil parametriert. Aufgrund unterschiedlicher örtlicher Randbedingungen können diese Kosten zwischen den einzelnen Ladepunkten variieren und dementsprechend unterschiedlich parametriert werden. Die einzelnen Anteile sind in Tabelle 4-15 dargestellt, wobei die Ausführungen keinen Anspruch auf Vollständigkeit erheben. Speziell für den Festanteil ist jeweils eine individuelle Betrachtung erforderlich.

Tabelle 4-15: Kostenbestandteile der streckenseitigen Ladeinfrastruktur

\begin{tabular}{cl}
\hline \multicolumn{1}{c}{$\begin{array}{c}\text { Festanteil } \\
\text { Einheit: EUR/LP }\end{array}$} & $\begin{array}{c}\text { Leistungsabhängiger Anteil } \\
\text { Einheit: EUR/kW/LP }\end{array}$ \\
\hline Kosten für Hochbau & $\begin{array}{l}\text { Dimensionierung der elektro- } \\
\text { technischen Komponenten nach } \\
\text { - Einhausung der Komponenten }\end{array}$ \\
- Lademast und Ladehaube bei & $\begin{array}{l}\text { der zu installierenden Leistung } \\
\text { der Ladepunkte }\end{array}$ \\
Kosten für Tiefbau & \\
- Einbau der Spulen in die Fahrbahn & \\
bei induktiven Systemen & \\
Netzanschlusskosten &
\end{tabular}


Unter Berücksichtigung der Festanteilkosten $K_{\text {fest }}$ und der leistungsabhängigen Kosten $k_{\text {var }}$ berechnen sich die Gesamtkosten für die Ladeinfrastruktur auf der Strecke $K_{\text {Ladepunkt,ges }}$ für eine bestimmte Anzahl an Ladepunkten $n_{\text {Ladepunkt }}$ aus der Summe über der Anzahl der einzelnen Ladepunkte $i$.

$$
K_{\text {Ladepunkt,ges }}=\sum_{i=0}^{n_{\text {Ladepunkt }}}\left(K_{\text {fest }, \mathrm{i}}+k_{\mathrm{var}, \mathrm{i}} \cdot P_{\text {Ladepunkt,i }}\right)
$$

Die Kostenbestandteile der streckenseitigen Ladeinfrastruktur gemäß Tabelle 4-15 können für jeden Ladepunkt individuell parametriert werden. So kann beispielsweise im Festanteil berücksichtigt werden, ob an einem potentiellen Ladepunkt bereits ein Zugang zum speisenden Energienetz vorhanden ist oder ob dieser erst aus einer entfernten Verteilstation zugänglich gemacht werden muss. Dementsprechend stellen sich unterschiedliche Kosten für den Netzanschluss ein. Des Weiteren ist über den Festanteil die Ladetechnologie parametrierbar. Bei konduktiven Systemen ist in der Regel kein Tiefbau erforderlich, während induktive Systeme oftmals nur einen begrenzten Hochbau erfordern.

Die notwendigen Kosten für Ladeinfrastruktur im Depot werden in dieser Arbeit nicht betrachtet. Aktueller Konsens von Betreibern und Herstellern ist, dass jedem Fahrzeug ein separates Ladegerät im Depot zugeordnet wird. Da im Rahmen dieser Arbeit die Anzahl der Fahrzeuge aufgrund des festen Fahrplans und damit der Taktzeit feststeht, würde die Berücksichtigung der Depot-Ladegeräte lediglich einen konstanten Offset auf alle technischen Konfigurationen bedeuten und damit nicht zur Unterscheidbarkeit einzelner Systemkonfigurationen beitragen.

\section{Energieverbrauch}

Der Energieverbrauch ist eine wesentliche Größe zur Beurteilung der Effizienz des Gesamtsystems Elektrobus. Der Kostenanteil des Energieverbrauchs wird in dieser Arbeit für den Einsatz eines Fahrzeugs an einem Betriebstag berechnet. Dabei muss neben dem Verbrauch des Fahrzeugs auch der Wirkungsgrad der Nachladung betrachtet werden. Anschließend wird der Energieverbrauch für alle Fahrzeuge inkl. Nachladung an einem Betriebstag hochskaliert. Der Gesamtenergieverbrauch $E_{\text {ges,Sys }}$ des Systems über dem betrachteten Lebenszyklus $L C$ ergibt sich aus der Summe der einzelnen Betriebstage $d$.

$$
E_{\mathrm{ges}, \mathrm{Sys}}=\sum_{d=1}^{L C}\left(E_{\mathrm{Fzg}} \cdot n_{\mathrm{Fzg}}+E_{\mathrm{Nachladung}} \cdot \eta_{\text {Nachladung }}\right)
$$


Die Energiekosten des Gesamtsystems $K_{\text {Energie,ges,Sys }}$ über dem Lebenszyklus berechnen sich unter Berücksichtigung der spezifischen Energiekosten $k_{\text {Energie }}(E U R / k W h)$.

$$
K_{\text {Energie,ges,Sys }}=k_{\text {Energie }} \cdot E_{\text {ges,Sys }}
$$

\section{Zusammenfassung}

In Tabelle 4-16 sind die einzelnen Bestandteile des Kostenmodells und die jeweiligen Einheiten zusammengefasst.

Tabelle 4-16: Kostenbestandteile und zugehörige Einheiten

\begin{tabular}{ll}
\hline Fahrzeuge ohne Traktionsbatterie & EUR/Fahrzeug \\
Traktionsbatterie & EUR/kWh \\
Ladeinfrastruktur Festanteil & EUR/Ladepunkt \\
Ladeinfrastruktur & EUR/kW \\
leistungsabhängiger Anteil & EUR/kWh \\
\hline
\end{tabular}





\section{Systemsimulation und Optimierung}

In diesem Kapitel werden die Funktionalität der entwickelten Simulationsumgebung mit integrierter Optimierungsfunktion vorgestellt sowie resultierende Sensitivitäten einzelner Systemparameter ermittelt. Dafür werden in den folgenden Abschnitten zunächst verschiedener Einsatzszenarien definiert, welche in Einzelsimulationen umgesetzt und hinsichtlich unterschiedlicher Aspekte ausgewertet werden. Anschließend werden Optimierungsrechnungen mit unterschiedlichen Prämissen und Sensitivitätsanalysen betrieblicher sowie ökonomischer Faktoren durchgeführt.

\subsection{Entwicklung verschiedener Einsatzszenarien}

\subsection{1 Überblick}

In diesem Abschnitt werden verschiedene Einsatzszenarien eingeführt. Als Grundlage dient ein Basisszenario, welches im weiteren Verlauf des Abschnitts hinsichtlich verschiedener betrieblicher und technischer Randbedingungen erweitert wird. In Kapitel 5.3 werden anschließend sowohl für das Basisszenario als auch für das erweiterte Szenario Optimierungsrechnungen durchgeführt. Eine Übersicht der Einsatzszenarien ist in Abbildung 5-1 gegeben.

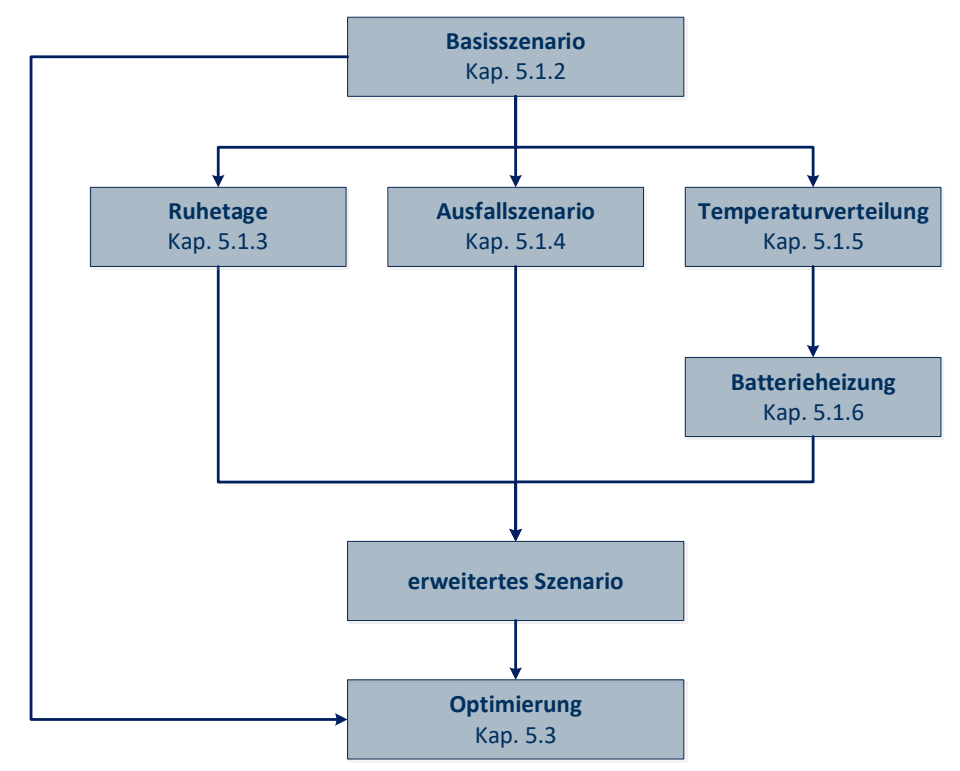

Abbildung 5-1: Übersicht Einsatzszenarien 


\subsubsection{Basisszenario}

Für alle folgenden Szenarien wird beispielhaft eine Buslinie in Dresden betrachtet. Das Höhenprofil der Strecke ist in Abbildung 5-2 für die Hin- und Rückrichtung gegeben. Die Strecke führt im ersten Teil durch eher flaches Terrain, bevor eine starke Steigung heraus aus dem Elbtal führt. Die Strecke hat eine Länge von insgesamt $38 \mathrm{~km}$ (Hin- und Rückfahrt) und eine max. Höhendifferenz von ca. 180 m. Die Simulation des Fahrzeugenergiebedarfs sowie der Fahrzeit wurde mittels des Modells in (Bunzel et al., 2018) durchgeführt. In Abbildung 5-3 ist der Geschwindigkeitsverlauf für einen Umlauf gegeben. Es ist erkennbar, dass das Fahrzeug eine Vielzahl an Haltestellen bedient und die Geschwindigkeit eine dementsprechende Dynamik aufweist. In der Mitte und am Ende des Fahrzyklus sind jeweils längere Standzeiten vorhanden. Hier befindet sich das Fahrzeug an den Wendepunkten der Strecke. Diese kommen als potentielle Ladezeiten in Frage.

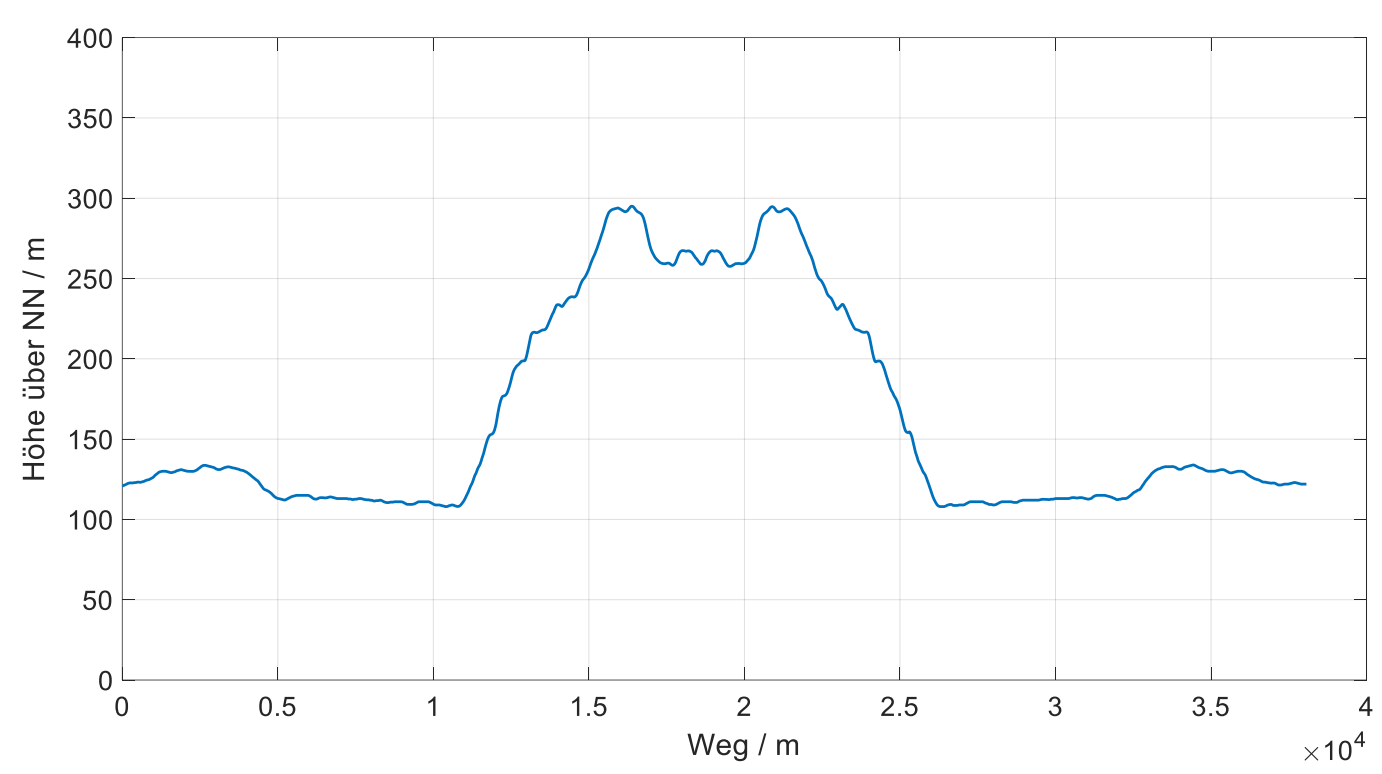

Abbildung 5-2: Höhenprofil eines Umlaufs

Der resultierende elektrische Leistungsverlauf des Fahrzeugs ist in Abbildung 5-4 gegeben. Darin ist die Dynamik des Geschwindigkeitsverlaufs wiedererkennbar. Der Leistungsbedarf wurde am Zwischenkreis ermittelt und berücksichtigt somit alle Wirkungsgrade der einzelnen Antriebskomponenten und Hilfsbetriebeeinrichtungen. Es wurde zunächst ein Fahrzeug ohne Batteriegewicht simuliert. Der Energieinhalt der Batterie ist ein Variationsparameter, wodurch die Leistungsbedarfssimulation für unterschiedliche Batteriegrößen aufgrund des sich ändernden Gewichts stets neu durchgeführt werden muss. Gemäß der Definition der Stromrichtung im elektrischen ESB 
(Abbildung 4-4) bedeutet eine positive Leistung eine Entladung, während eine negative Leistung für eine Ladung der Batterie steht.

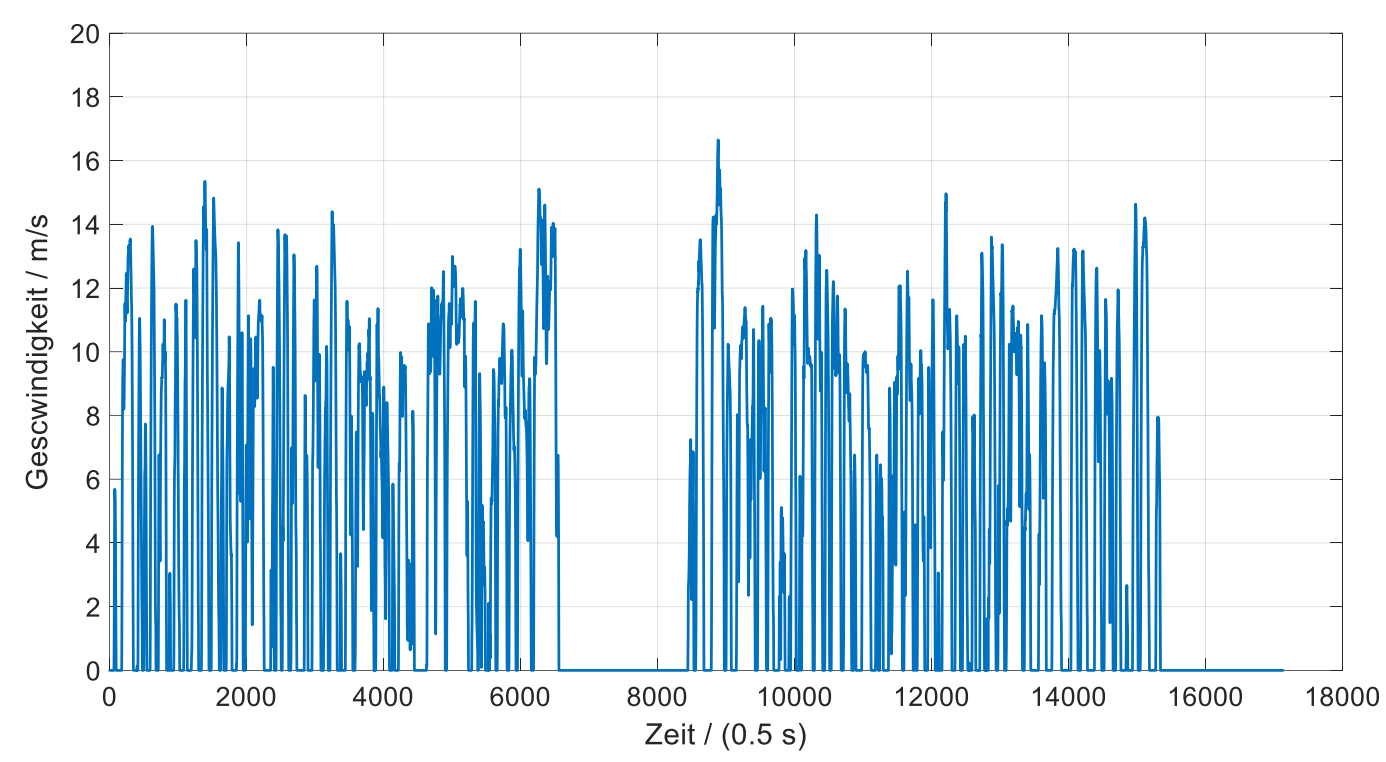

Abbildung 5-3: Simulierter Geschwindigkeitsverlauf eines Umlaufs

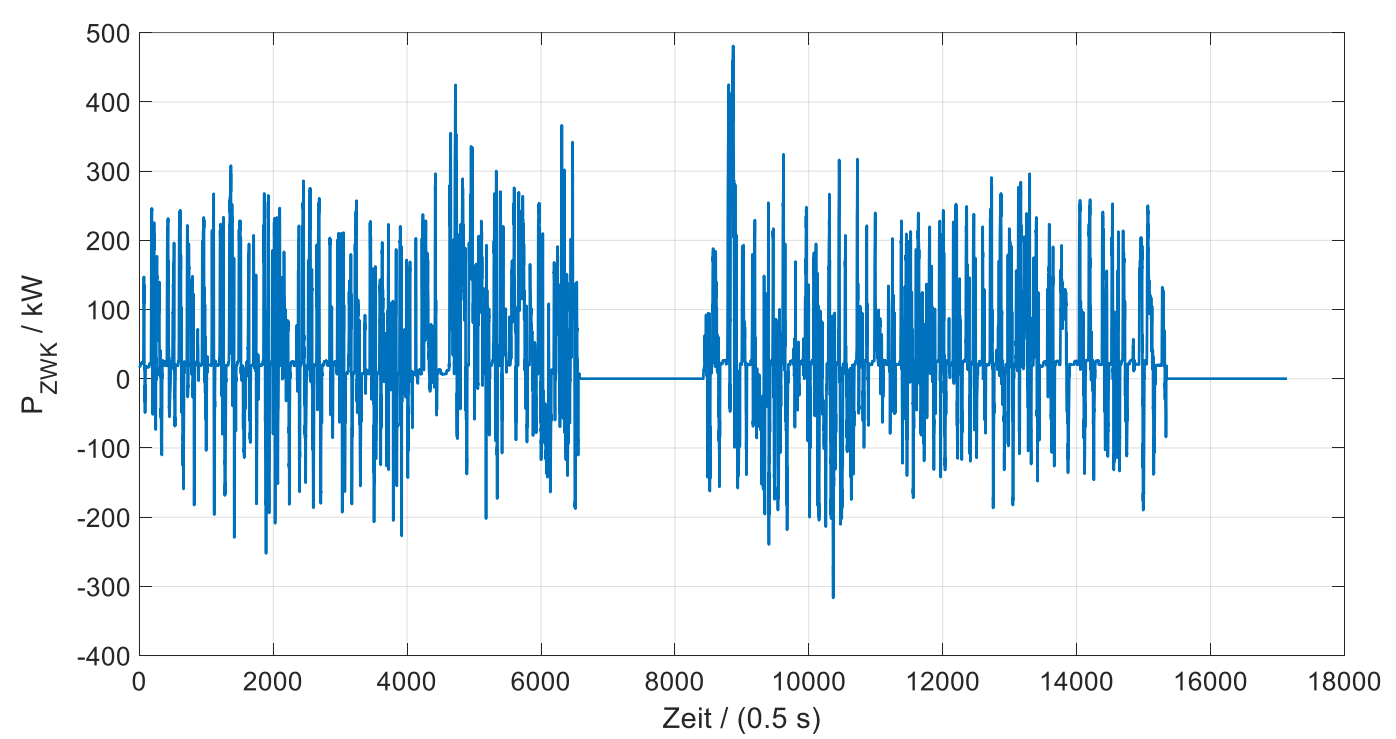

Abbildung 5-4: Simulierter elektrischer Leistungsbedarf am Zwischenkreis eines Umlaufs (Fahrzeug ohne Batteriegewicht)

Im nächsten Schritt wird die Traktionsbatterie soweit elektrisch konfiguriert, dass anschließend lediglich der Variationsparameter des Energieinhalts (gesteuert über die 
Anzahl paralleler Zellen) übrigbleibt. Als Spannungslage wird in dieser Arbeit das Nennspannungsniveau des Pilotprojektes der Linie 79 aus Dresden auf Basis der in Kapitel 4.1 eingeführte $\mathrm{LiFePO}_{4}$ Zelle von A123 Systems verwendet. Deren Spannungsbereiche sind wie folgt charakterisiert (Tabelle 5-1).

\section{Tabelle 5-1: Spannungsbereich der $\mathrm{LiFePO}_{4}$ Zelle von $\mathrm{A} 123$ Systems}

\begin{tabular}{ll}
\hline Nennspannung & $3,3 \mathrm{~V}$ \\
Arbeitsbereich & $2,0 \mathrm{~V}-3,6 \mathrm{~V}$ \\
\hline
\end{tabular}

Im Fahrzeug der Linie 79 sind insgesamt 208 Zellen in Reihe geschaltet. Damit ergeben sich folgende Spannungsbereiche des Batteriesystems (Tabelle 5-2).

Tabelle 5-2: Spannungsbereich des Batteriesystems

\begin{tabular}{ll}
\hline Nennspannung & $687 \mathrm{~V}$ \\
Minimale Spannung & $416 \mathrm{~V}$ \\
Maximale Spannung & $750 \mathrm{~V}$ \\
\hline
\end{tabular}

Dieser Spannungsbereich des Batteriesystems und damit die Anzahl serieller Zellen bleiben für die Betrachtungen in dieser Arbeit konstant. Grundsätzlich können jedoch auch beliebige andere Spannungslagen parametriert werden. Die Variation des Batterieenergieinhalts wird nun über die Anzahl paralleler Stränge realisiert. Der kleinste darstellbare Energieinhalt ist bei einem parallelen Strang gegeben und beträgt 13,5 kWh. Für das Basisszenario wird eine Batteriekonfiguration mit 10 parallelen Zellsträngen definiert, was einem nominellen Energieinhalt von $135 \mathrm{kWh}$ entspricht. Es wird angenommen, dass eine Nachladung an beiden Wendepunkten möglich ist. Die verfügbare Ladeleistung am Fahrzeug wird mit $200 \mathrm{~kW}$ je Ladepunkt definiert. Die elektrischen Kenndaten der Batterie sind in Tabelle 5-3 zusammengefasst. Für den Aufbau eines Batteriemoduls wird eine Konfiguration mit 13 Zellen pro Modul verwendet. Es wird angenommen, dass die Zellen in x-Richtung gestapelt sind (Vgl. Abbildung 5-5). Für die Kühlung wird für alle in dieser Arbeit betrachteten Szenarien ein Wärmeübergangskoeffizient von $100 \mathrm{~W} /\left(\mathrm{m}^{2} \mathrm{~K}\right)$ nach Tabelle 4-6 verwendet, was einer forcierten Luftkühlung entspricht. Es wird idealisierter Weise davon ausgegangen, dass alle Außenseiten eines Moduls gleichmäßig gekühlt werden.

Neben der elektrischen und thermischen Grundkonfiguration werden für das Basisszenario betriebliche Randbedingungen definiert, welche ebenfalls in Tabelle 5-3 zusammengefasst sind. 
Tabelle 5-3: Betriebliche und technische Daten und Randbedingungen des Basisszenarios

\begin{tabular}{ll}
\hline Fahrzeugtyp & $18 \mathrm{~m}$ Gelenkbus \\
Umlauflänge & $38 \mathrm{~km}$ \\
Anzahl der Umläufe pro Tag & 6 \\
Tagesfahrleistung & $6 \cdot 38 \mathrm{~km}=228 \mathrm{~km}$ \\
Takt & $20 \mathrm{~min}$ \\
Möglichkeit zur strecken- & an beiden \\
seitigen Nachladung & Wendepunkten \\
max. Ladezeit LP 1 & 15 min \\
max. Ladezeit LP 2 & 10 min \\
Umgebungstemperatur & $15{ }^{\circ} \mathrm{C}$ (konstant) \\
Fahrzeuglebenszyklus & $12 \mathrm{Jahre}$ \\
parallele Zellstränge & 10 \\
Energieinhalt der Batterie & $135 \mathrm{kWh}$ \\
Ladeleistung & $200 \mathrm{~kW}$ \\
max. SOC-Bereich & $0,9-0,1$ \\
\hline
\end{tabular}

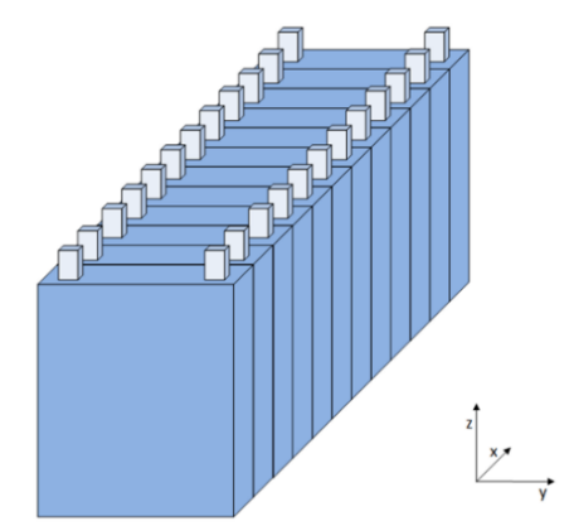

Abbildung 5-5: Schemenhafte Darstellung der geometrischen Zellanordnung in einem Modul, nach (Kritzinger, 2018b)

Für das definierte Basisszenario ergibt sich ein SOC-Verlauf gemäß Abbildung 5-6. Darin ist in blau der SOC-Verlauf für eine neue Batterie eingetragen. In rot ist der SOCVerlauf für eine gealterte Batterie dargestellt. Es ist erkennbar, dass diese im gealterten Zustand bereits vor der ersten Zwischenladung tiefer entladen wird. Dies ist vor allem 
auf die geringere verfügbare Kapazität in Folge der Alterung zurückzuführen. Mit zunehmendem Verlauf des Tages wird bei gealterter Batterie der maximale SOC von 0,9 nicht mehr erreicht, wohingegen eine neue stets auf diesen SOC aufgeladen werden kann. In Abbildung 5-7 ist der simulierte Verlauf der Batteriealterung aufgetragen. Es ist erkennbar, dass das EOL-Kriterium der Kapazität $\left(0,8 \cdot C_{0}\right)$ nach 1636 Tagen erreicht wird. In dieser Zeit steigt der Innenwiderstand hingegen nur auf das ca. 1,45-fache des initialen Wertes an. Das EOL-Kriterium von $2 \cdot R_{\mathrm{i}}$ wird in dieser Zeit nicht erreicht.

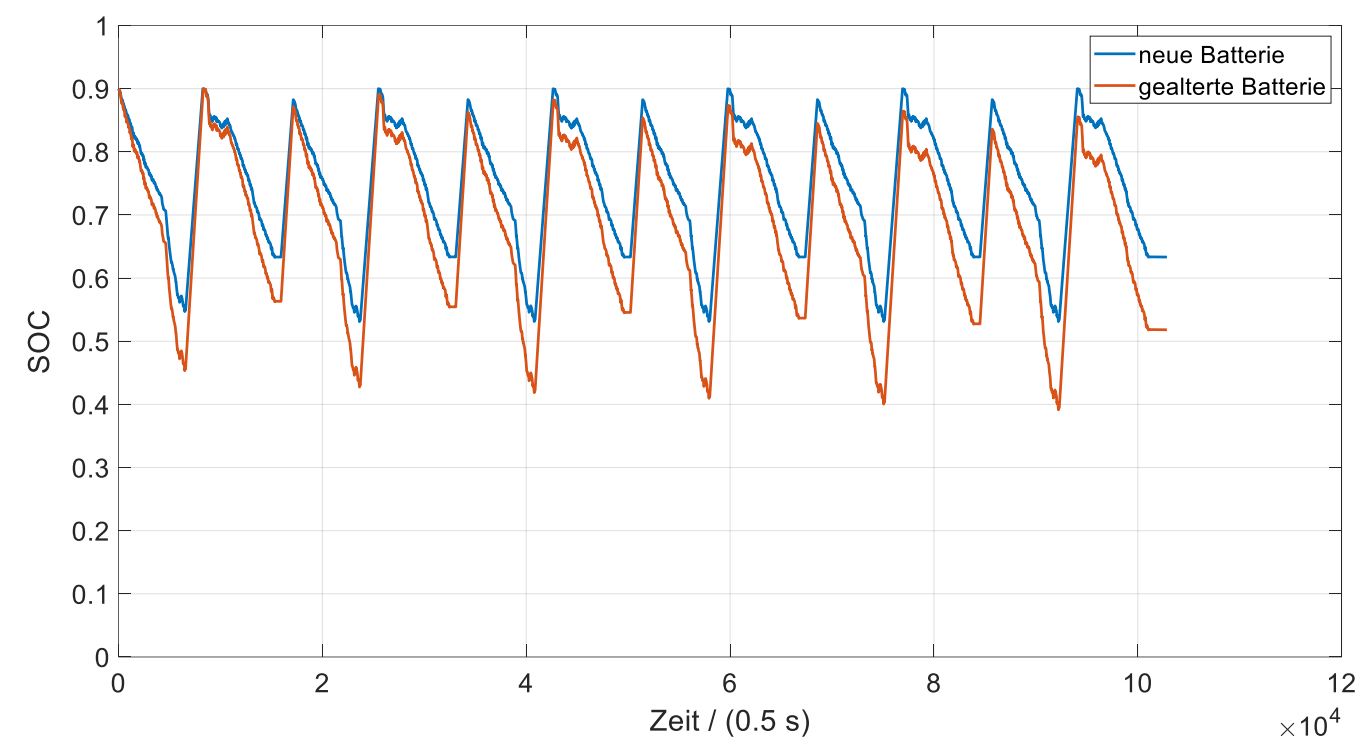

\section{Abbildung 5-6: SOC-Verlauf Basisszenario (10 parallele Stränge, 135 kWh, 200 kW)}

Der Verlauf der beiden Alterungskurven in Abbildung 5-7 erscheint auf den ersten Blick nahezu linear. Dies deckt sich mit der linearen Alterungsmodellierung aus Kapitel 4.1.5. Bei genauerer Betrachtung ergibt sich jedoch ein differenziertes Bild. In Abbildung 5-8 sind die numerisch differenzierten Verläufe der Kurven dargestellt. Dabei wurde stets die Differenz zweier aufeinander folgender Zeitschritte berechnet. Es ist erkennbar, dass die Alterungszunahme sowohl der Kapazität als auch des Innenwiderstands nicht linear verlaufen. Beide Kurven weisen ein exponentielles Verhalten auf. Dies ist mittels des Simulationsaufbaus (Abbildung 3-1) begründbar. Als Eingang in das Modell dient ein elektrischer Leistungsverlauf. Dieser ist bei konstanten Umgebungs- und Belastungsbedingungen ebenfalls konstant. Dagegen verändert sich die Leistungsfähigkeit der Batterie aufgrund der Alterung. Durch den höheren Innenwiderstand bricht die Klemmenspannung entsprechend schneller ein, wodurch sich bedingt durch die Leistungsanforderung des Fahrzeugs ein höherer Strom einstellt und somit die thermische Belastung steigt. Zusätzlich sinkt die Ruhespannung der Batterie aufgrund der reduzierten Kapazität schneller ab, wodurch der Anstieg des Batteriestroms nochmals verstärkt wird. Bei der Batteriealterung in einem Elektrobus handelt es sich somit um 
einen sich selbstverstärkenden, wenn auch schwach ausgeprägten Effekt, was mittels Abbildung 5-8 bestätigt wird. Darin ist der Alterungsverlauf aus Abbildung 5-7 in numerisch differenzierter Form gegeben. Es ist deutlich die steigende Geschwindigkeit der Alterung mit fortschreitender Zeit erkennbar. Dieser Zusammenhang gilt sowohl für die Kapazität als auch für den Innenwiderstand.
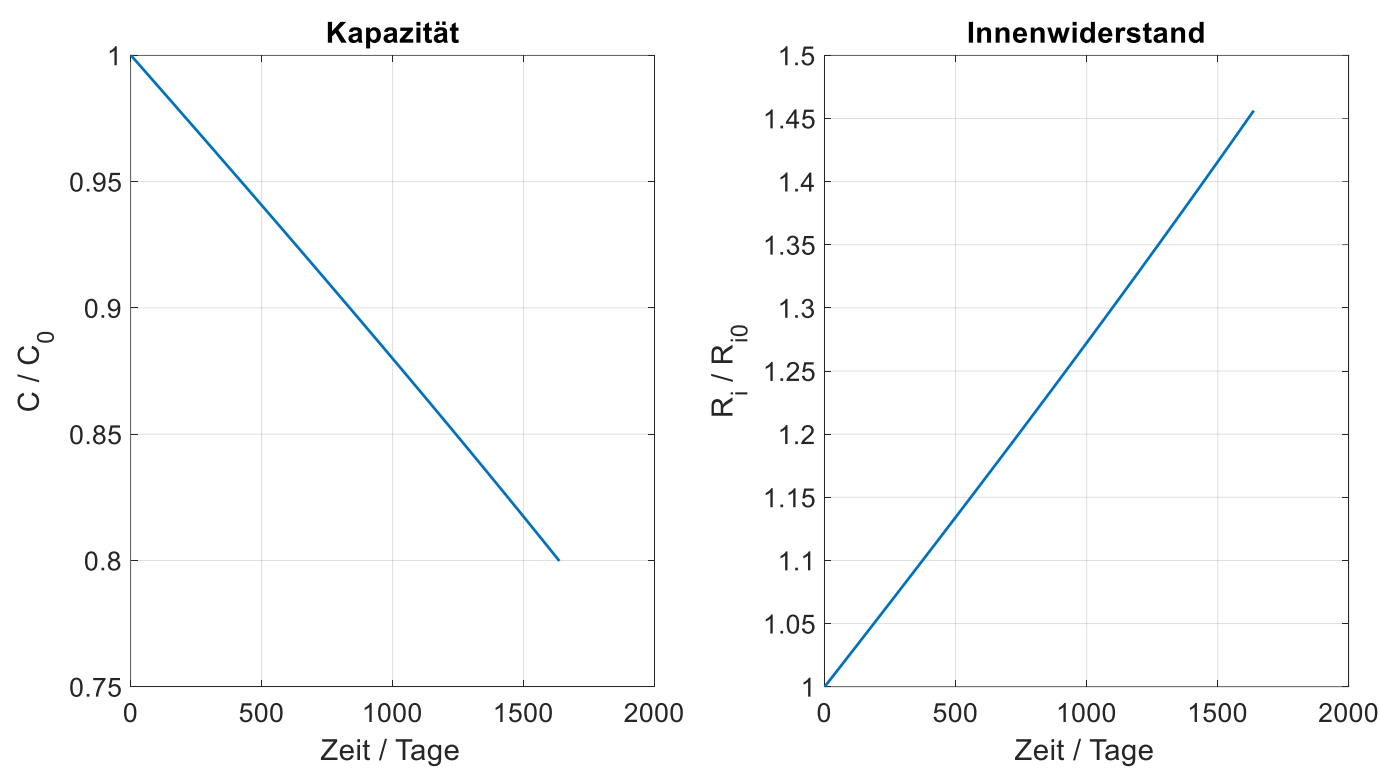

Abbildung 5-7: Alterungsverläufe im Basisszenario
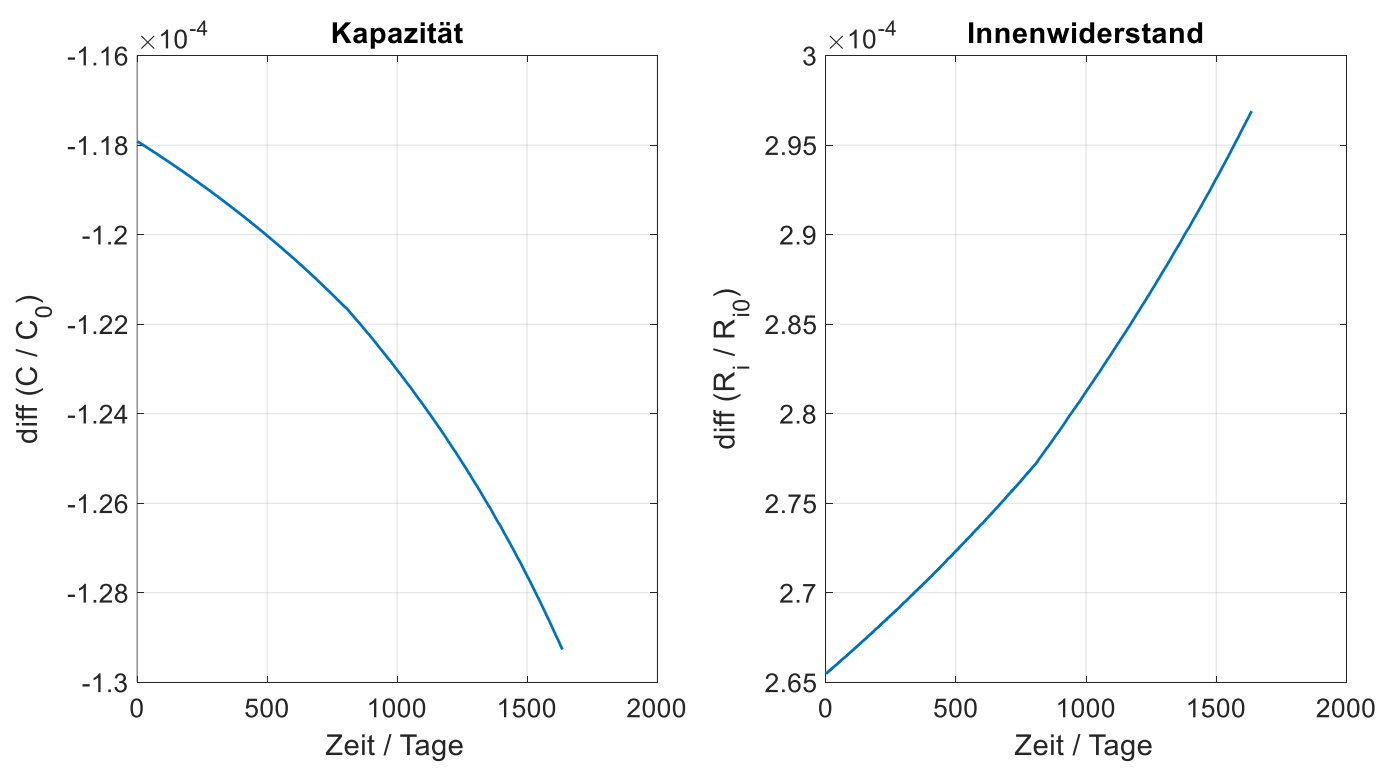

Abbildung 5-8: Numerisch differenzierte Alterungsverläufe im Basisszenario 


\subsubsection{Betriebs- und Ruhetage}

Die Simulation des Basisszenarios in Kapitel 5.1.2 basiert auf der Annahme, dass die Fahrzeuge jeden Tag das gleiche Betriebsprogramm absolvieren. Daraus ergäbe sich je nach täglicher Fahrstrecke eine sehr hohe jährliche Laufleistung. Im Basisszenario würde dies einer Laufleistung von $83.220 \mathrm{~km}$ pro Jahr entsprechen. Aufgrund von Wartungs- und Reparaturarbeiten sind die Fahrzeuge jedoch oftmals weniger als 365 Tage pro Jahr im Einsatz. Es wird demzufolge ein Einsatzszenario entworfen, in welchem zwischen Einsatztagen und Ruhetagen der Fahrzeuge unterschieden wird. Dies ist vor allem für die Berechnung der Batteriealterung wichtig, da an Ruhetagen keine zyklische, sondern nur kalendarische Alterung auftritt. Dafür wurde ein Parameter eingefügt, welcher aller $X$ Tage einen Ruhetag in das Betriebsprogramm einfügt. Ein beispielhaftes Betriebsprogramm mit einem Ruhetag aller 7 Tage $(X=7)$ ist in Tabelle 5-4 gegeben.

Tabelle 5-4: beispielhaftes Betriebsprogramm

\begin{tabular}{ccccccc}
\hline Mo & Di & Mi & Do & Fr & Sa & So \\
Betrieb & Betrieb & Betrieb & Betrieb & Betrieb & Betrieb & Ruhe \\
\hline
\end{tabular}
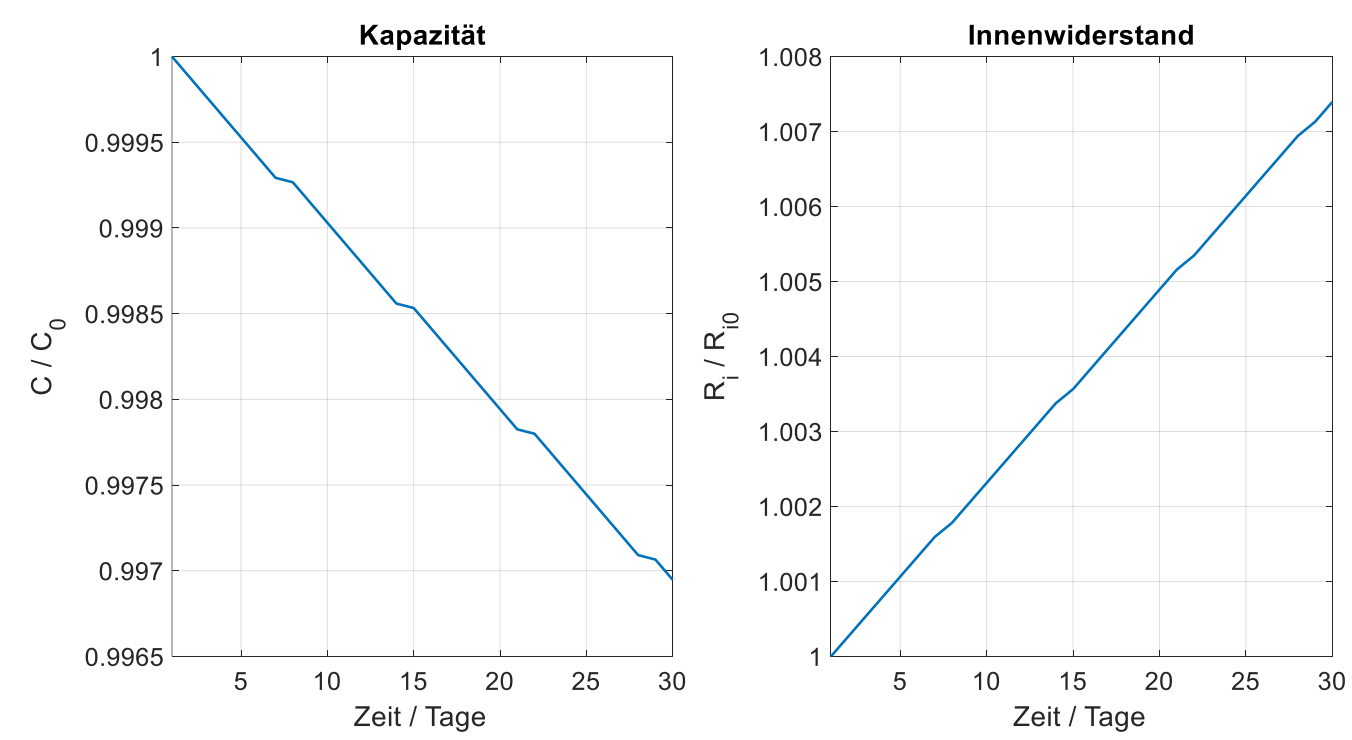

Abbildung 5-9: Verlauf der Alterung für 30 Tage bei einem Ruhetag pro Woche

Grundsätzlich sind an dieser Stelle beliebige Konstellationen für Betriebsprogramme darstellbar. Eine Differenzierung der täglichen Laufleistung an Betriebstagen erfolgt im Rahmen dieser Arbeit nicht. Im Folgenden wird gezeigt, wie sich Ruhetage auf die Alterung der Batterie auswirken. Die Darstellungen sind dabei immer in Relation zum 
Szenario ohne Ruhetage (365 Betriebstage pro Jahr) zu sehen. In Abbildung 5-9 ist die deutlich stärkere Alterung an Betriebstagen gegenüber dem Ruhetag erkennbar, da zusätzlich zur kalendarischen Alterung auch zyklische Alterungseffekte auftreten. Dies gilt sowohl für die Abnahme der Kapazität als auch für den Anstieg des Innenwiderstands. Es ist ebenfalls erkennbar, dass der Unterschied zwischen kalendarischer und zyklischer Alterung bei der Kapazität deutlicher ausfällt als beim Innenwiderstand.

Tabelle 5-5: Auswirkung der Ruhetage pro Jahr auf die Alterung der Batterie

\begin{tabular}{cccccc}
\hline $\begin{array}{c}\text { aller X Tage } \\
\text { ein Ruhetag }\end{array}$ & $\begin{array}{c}\text { Fahrzeuglauf- } \\
\text { leistung pro Jahr }\end{array}$ & \multicolumn{2}{c}{$\begin{array}{c}\text { Betriebstage } \\
\text { pro Jahr }\end{array}$} & \multicolumn{2}{c}{ Lebensdauer } \\
\hline kein Ruhetag & $83220 \mathrm{~km}$ & 365 & $100 \%$ & 1636 Tage & $100 \%$ \\
7 & $71331 \mathrm{~km}$ & 313 & $86 \%$ & 1841 Tage & $113 \%$ \\
6 & $69350 \mathrm{~km}$ & 304 & $83 \%$ & 1881 Tage & $115 \%$ \\
5 & $66576 \mathrm{~km}$ & 292 & $80 \%$ & 1939 Tage & $119 \%$ \\
4 & $62415 \mathrm{~km}$ & 274 & $75 \%$ & 2033 Tage & $124 \%$ \\
3 & $55480 \mathrm{~km}$ & 243 & $67 \%$ & 2213 Tage & $135 \%$ \\
\hline
\end{tabular}

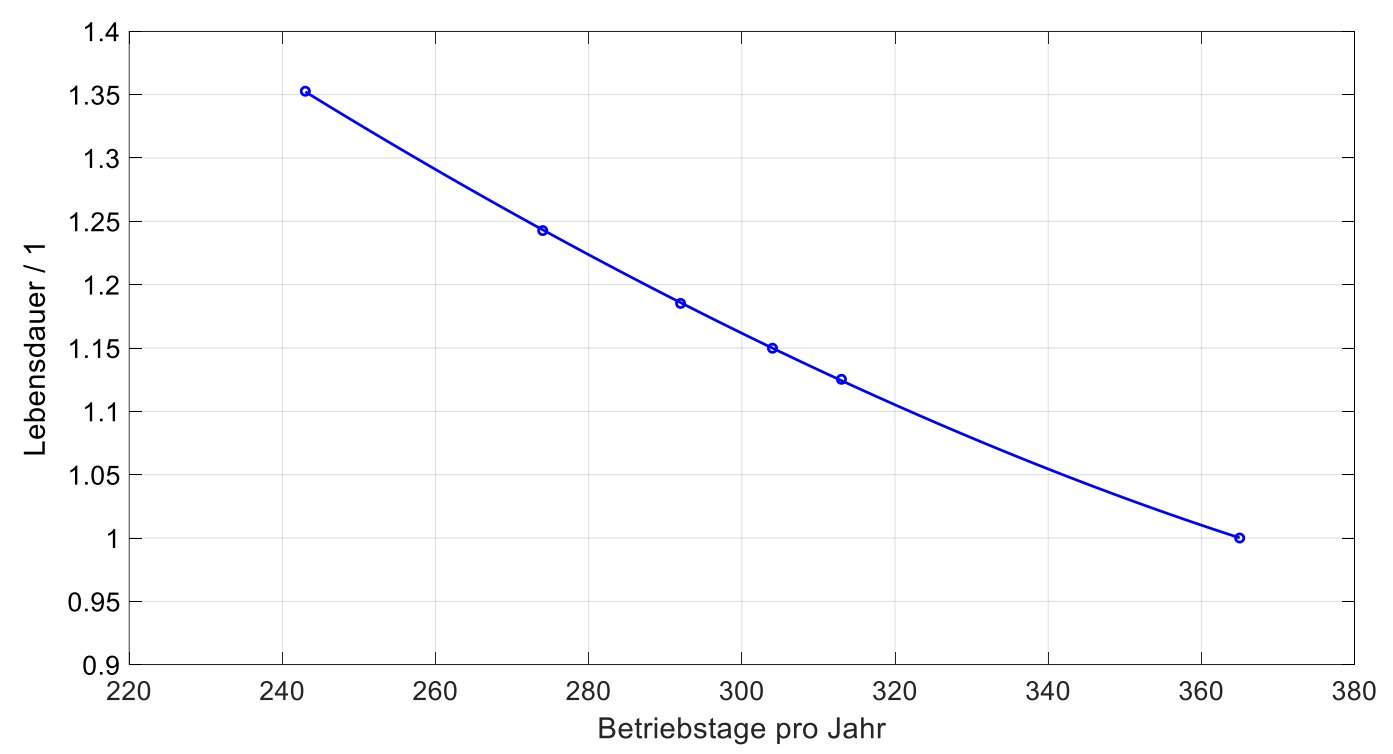

Abbildung 5-10: Lebensdauer der Batterie in Abhängigkeit der jährlichen Betriebstage 
Im Folgenden wird nun die Auswirkung der Anzahl jährlicher Ruhetage auf die zu erwartende Lebensdauer der Batterie untersucht. Die Ergebnisse der Berechnungen sind in Tabelle 5-5 dargestellt und in Abbildung 5-10 veranschaulicht. Daraus geht hervor, dass ein schwach exponentiell ausgeprägter Zusammenhang besteht. Die Lebensdauer der Batterie erhöht sich demnach leicht überproportional zur Reduzierung der jährlichen Betriebstage. Es kann demnach geschlussfolgert werden, dass eine lineare Skalierung von Lebensdauer und Laufleistung zu falschen Ergebnissen führt.

\subsubsection{Ausfallszenario}

In den bisherigen Betrachtungen wurde stets davon ausgegangen, dass alle vorgesehenen Ladephasen vollständig zur Verfügung stehen. Als Ladephase wird ein Ladevorgang an einem der Ladepunkte während der dort verfügbaren Ladezeit definiert. In realen Anwendungen muss jedoch immer auch mit Verspätungen im Betriebsablauf gerechnet werden. Dies führt dazu, dass Ladephasen nur mit reduzierter Ladezeit zur Verfügung stehen oder gar ganz entfallen. Derartige Szenarien sind wichtig für die Beurteilung der Qualität des Betriebsablaufes mit Elektrobussen. Dabei hat die Einhaltung des Fahrplans oberste Priorität. Im Rahmen dieser Arbeit werden solche Verspätungsszenarien derart berücksichtigt, dass der Ausfall ganzer Ladephasen simuliert wird. In einem solchen Fall würde das Fahrzeug in der Realität den Wendepunkt erreichen und anschließend direkt wieder verlassen. Da in dieser Arbeit ein Rückwärtsmodell zur Anwendung kommt und somit keine Rückwirkungen auf Fahrdynamik und Fahrzeit berechnet werden, wird ein Ausfallszenario derart simuliert, dass das Fahrzeug am Wendepunkt steht und keine Ladung erfolgt. Für die Beurteilung der energetischen Bilanz der Batterie ist dieses Vorgehen nahezu äquivalent zur längeren Fahrzeit. Die Berücksichtigung von Ausfallszenarien ist aus zwei Gründen wichtig. Zum einen wird damit die Anzahl technisch realisierbarer Konfiguration erheblich eingeschränkt, da das System gegenüber dem Basisszenario zu einem gewissen Grad überdimensioniert werden muss. Zum anderen wird aufgrund des dann größeren SOCHubs die Alterung der Batterie beeinflusst.

Wird nun für das Basisszenario der Ausfall einer Ladephase berücksichtigt, so ist diese Systemkonfiguration nicht mehr vollständig umsetzbar. Ein Fahrzeug mit einer neuen Batterie würde die geforderte Tageslaufleistung erbringen können. Mit einer vollständig gealterten Batterie (80\% Kapazität, 200\% Innenwiderstand) kann das Tagesprogramm hingegen nicht bewältigt werden. Der resultierende SOC-Verlauf ist in Abbildung 5-11 dargestellt.

Mit einer Erhöhung der Anzahl paralleler Zellen von 10 auf 12 wird eine technisch realisierbare Konfiguration auch bei gealterter Batterie erreicht. In Abbildung 5-12 ist der SOC-Verlauf für eine Batterie mit 12 parallelen Zellen (entspricht $162 \mathrm{kWh}$ ) bei Ausfall einer Ladephase gezeigt. Es ist erkennbar, dass sich dadurch ein größerer SOC-Hub 
ergibt. Gemäß des Alterungsmodells wird erwartet, dass dies eine negative Auswirkung auf die Alterung im Vergleich zum Basisszenario ohne Ausfall einer Ladephase hat.

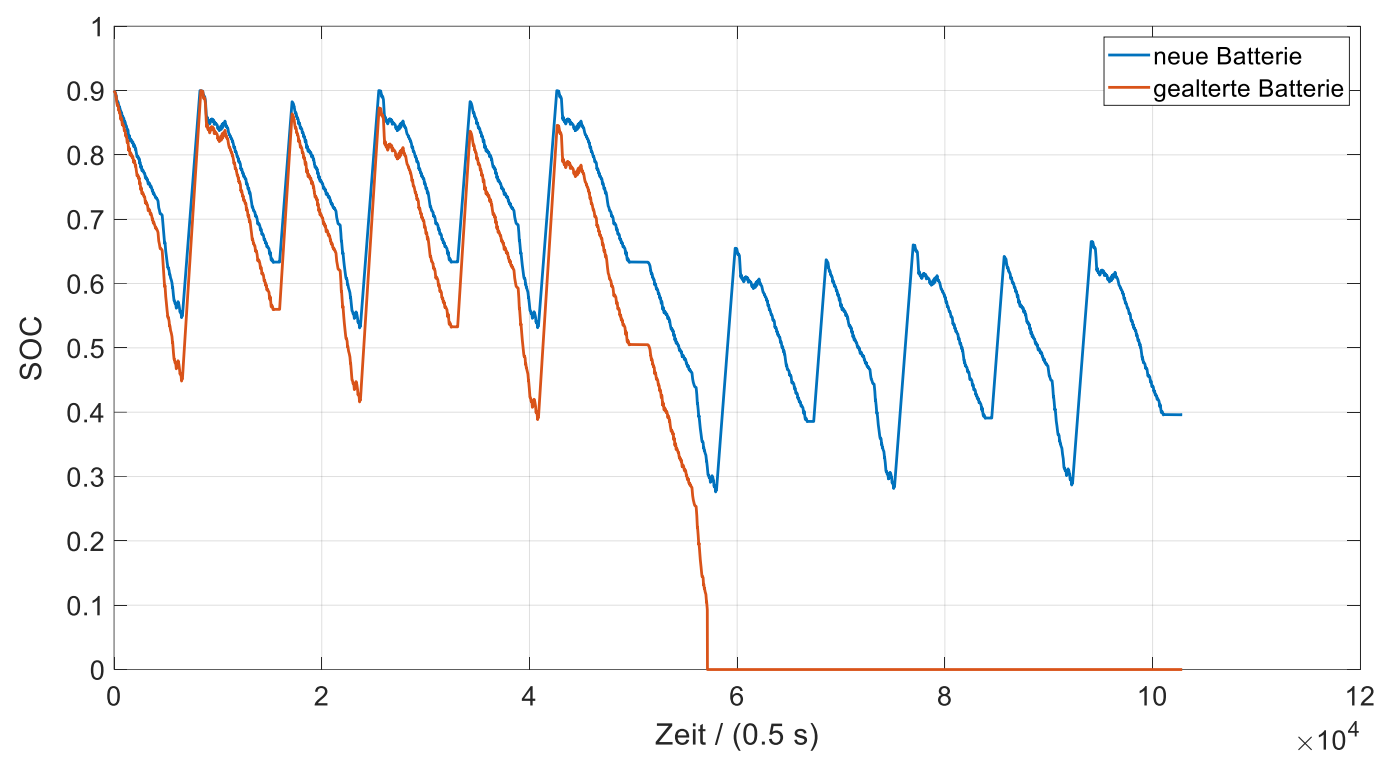

Abbildung 5-11: SOC-Verlauf des Basisszenarios mit Ausfall einer Ladephase

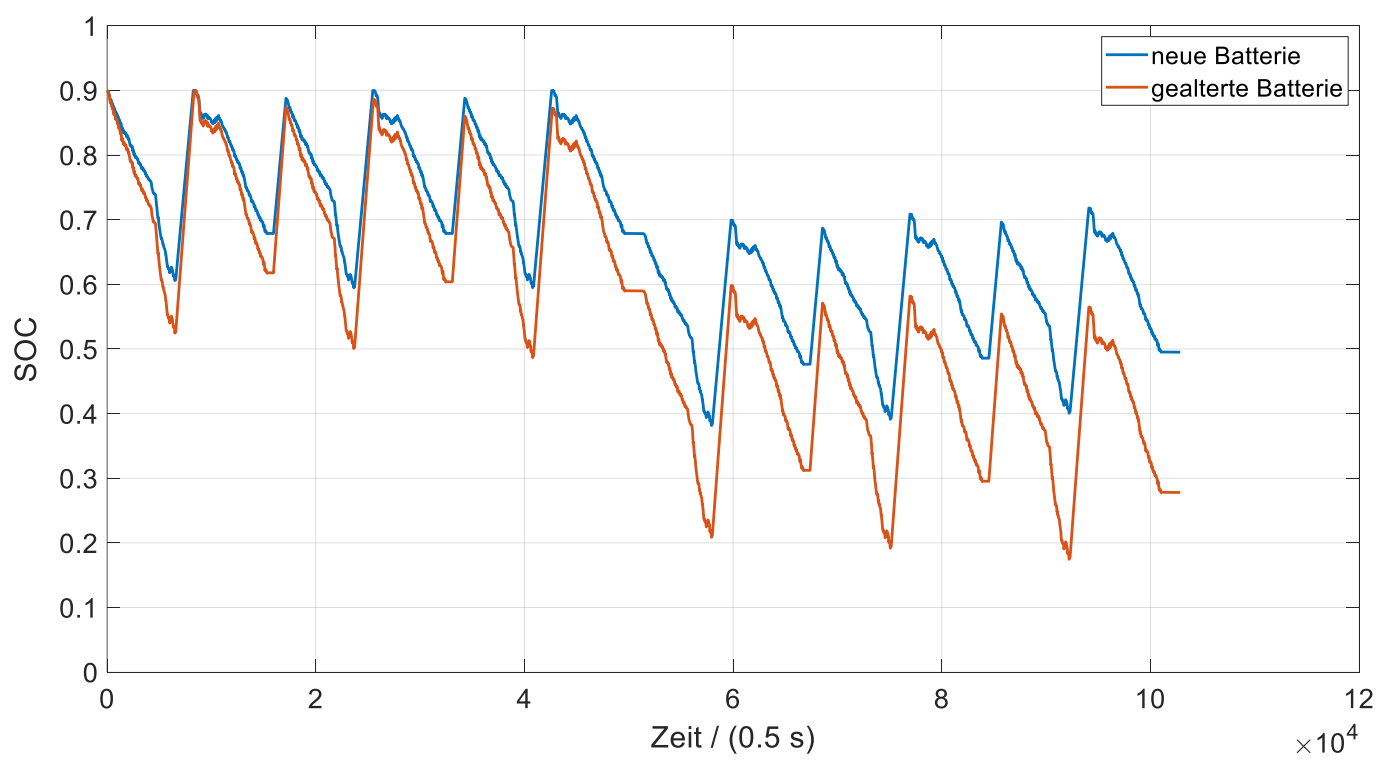

Abbildung 5-12: SOC-Verlauf mit Ausfall einer Ladephase (162 kWh, $200 \mathrm{~kW}$ ) 
Im Folgenden wird untersucht, wie groß der Einfluss von Ausfallszenarien auf die Batteriealterung ist. Ziel der Untersuchung ist die Ermittlung des Zusammenhangs zwischen der Anzahl an Tagen mit Ausfallszenario je Woche und der resultierenden Alterung. Die Ergebnisse sind in Tabelle 5-6 dargestellt und in Abbildung 5-13 veranschaulicht.

\section{Tabelle 5-6: Auswirkung der Anzahl an Tagen mit Ausfallszenario auf die Lebensdauer der Batterie}

\begin{tabular}{ccc}
\hline $\begin{array}{c}\text { Anzahl der Tage mit } \\
\text { Ausfallszenario pro } \\
\text { Woche }\end{array}$ & \multicolumn{2}{c}{ Lebensdauer } \\
\hline 0 & 1931 Tage & $100 \%$ \\
1 & 1906 Tage & $98,7 \%$ \\
2 & 1883 Tage & $97,5 \%$ \\
3 & 1860 Tage & $96,3 \%$ \\
4 & 1838 Tage & $95,2 \%$ \\
5 & 1816 Tage & $94,0 \%$ \\
6 & 1795 Tage & $93,0 \%$ \\
7 & 1774 Tage & $91,9 \%$ \\
\hline
\end{tabular}

Die Simulationsergebnisse zeigen einen schwach exponentiell ausgeprägten Zusammenhang. Ein Tag mit Ausfallszenario pro Woche bewirkt dabei nur eine Reduzierung der Lebensdauer von 1,3\%. Auch bei der Annahme, dass an allen Wochentagen ein Ausfallszenario vorhanden ist, wird die zu erwartende Lebensdauer um lediglich rund $8 \%$ reduziert. Im Vergleich zu den Ergebnissen der Betrachtung der Ruhetage (14\% erhöhte Lebensdauer bei einem Ruhetag aller 7 Tage, Vgl. Kap. 5.1.3) kann die Reduzierung aufgrund von Ausfallszenarien als moderat eingestuft werden. Dennoch ist es aus Betreibersicht wichtig, die Umlaufzeiten der Linie möglichst stabil zu halten und somit eine beschleunigte Alterung aufgrund von Verspätungen zu vermeiden.

Es kann demnach festgehalten werden, dass Ausfallszenarien aus Batteriealterungssicht eine untergeordnete Rolle in der Systemauslegung spielen. Vielmehr ist es jedoch wichtig, dass die Batterie und die Nachladung derart dimensioniert werden, dass ein qualitativer Betriebsablauf auch bei Verspätungen sichergestellt werden kann. Im hier gezeigten Beispiel konnte dies erst bei einer Erhöhung der Batteriekapazität im Vergleich zum Basisszenario erreicht werden. 


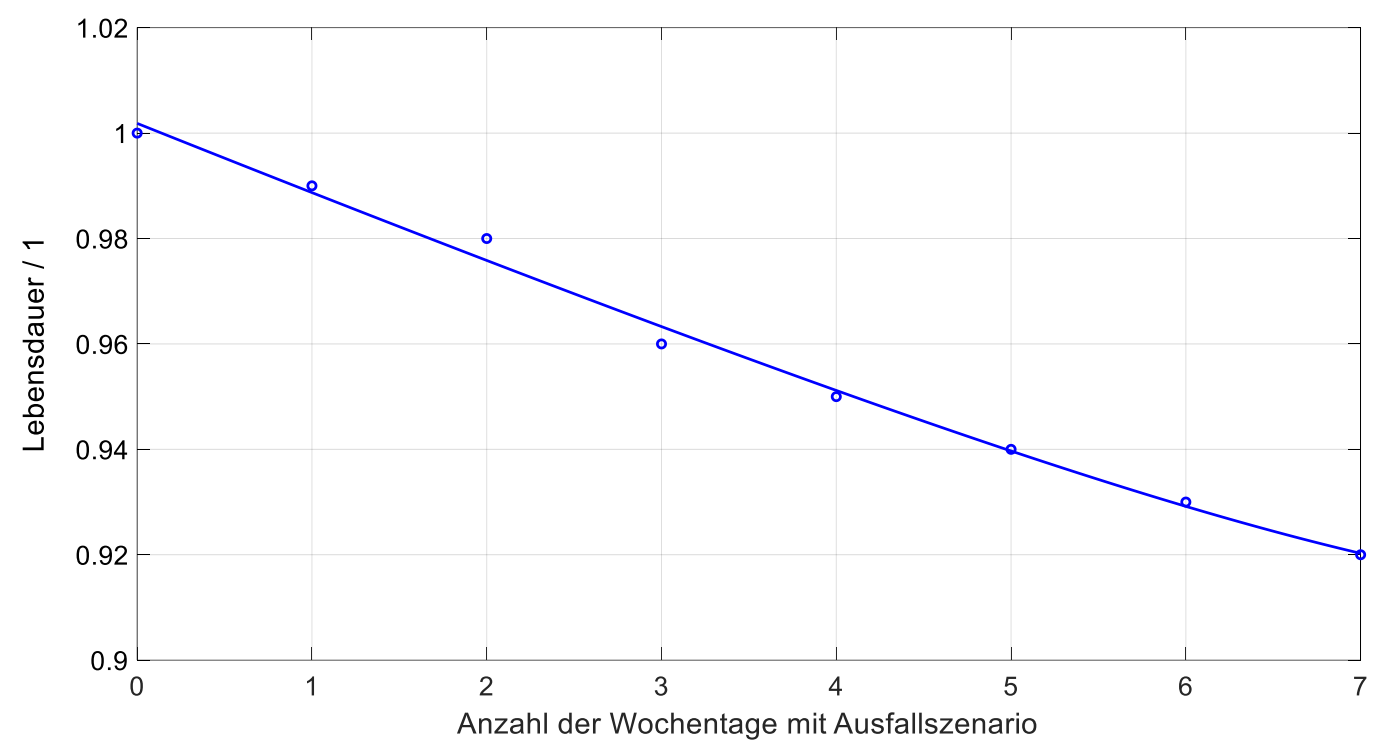

Abbildung 5-13: Batterielebensdauer in Abhängigkeit der Anzahl an Wochentagen mit Ausfallszenario

\subsubsection{Temperaturverteilung}

Im Basisszenario sowie allen weiteren bisherigen Untersuchungen wurde stets eine konstante Umgebungstemperatur von $15^{\circ} \mathrm{C}$ angenommen. Aus Sicht der Batterieperformance (Temperaturabhängigkeit der Kapazität) und der Alterung (10-K-Regel) führt die Annahme einer konstanten Umgebungstemperatur zu einem methodischen Fehler. In diesem Abschnitt wird nun der Einfluss der Umgebungstemperatur auf die Batterieperformance und die resultierende Alterung untersucht. Dabei werden verschiedene Granularitätsstufen der Temperaturverteilung betrachtet:

- $25^{\circ} \mathrm{C}$ konstant,

- $15^{\circ} \mathrm{C}$ konstant,

- $10^{\circ} \mathrm{C}$ konstant,

- Monatsmittelwerte, und

- Tagesmittelwerte.

Für die Monats- und Tagesmittelwerte wurden Daten des Deutschen Wetterdienstes verwendet. Die Verläufe sind in Abbildung 5-14 dargestellt. Die Monatsmittelwerte gelten für Deutschland und wurden im Zeitraum von 2001 bis 2017 erhoben. Der Verlauf der Tagesmittelwerte entspricht dem Jahr 1991 und wurde an der Wetterstation 
Dresden-Klotzsche gemessen. Die Verläufe wurden bewusst ausgewählt, da beide annähernd gleich große Mittelwerte aufweisen:

- Mittelwert der Monatsmittelwerte: $8,9^{\circ} \mathrm{C}$,

- Mittelwert der Tagesmittelwerte: $9,1^{\circ} \mathrm{C}$.
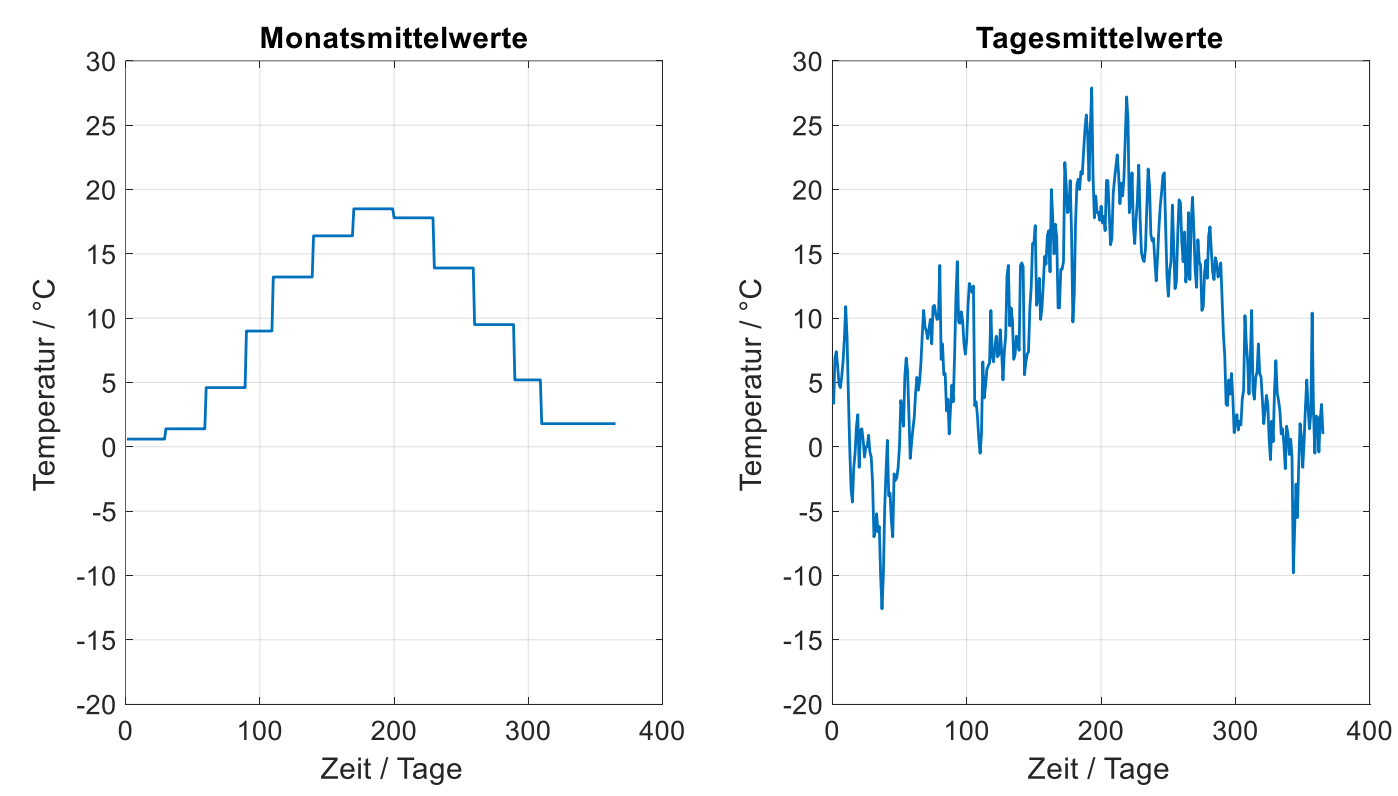

\section{Abbildung 5-14: Temperaturverläufe, links: Monatsmittelwerte für Deutschland (2001-2017), rechts: Tagesmittelwerte der Station Dresden-Klotzsche (1991)}

Zunächst wird die technische Realisierbarkeit des Basisszenarios bei veränderter Umgebungstemperatur überprüft (bisher: $15^{\circ} \mathrm{C}$ konstant). Aufgrund der Temperaturabhängigkeit der Batterie wird zunächst ein Tagesumlauf mit der niedrigsten Temperatur aller Granularitätsstufen simuliert. Diese ist im Verlauf der Tagesmittelwerte zu finden und wurde am 06.02.1991 mit $-12.6^{\circ} \mathrm{C}$ gemessen.

Das Simulationsergebnis in Abbildung 5-15 zeigt, dass aufgrund der reduzierten verfügbaren Kapazität (Vgl. Abbildung 4-6) der Tagesumlauf mit der Konfiguration aus dem Basisszenario bereits mit einer neuen Batterie nicht realisierbar ist. Im zweiten Umlauf des Tages wird die Grenze des minimalen SOC erreicht, sodass weniger als ein Drittel der geforderten Tagesfahrleistung absolviert werden kann. Eine technisch realisierbare Konfiguration für eine neue und eine gealterte Batterie wird bei einer Umgebungstemperatur von $-12,6^{\circ} \mathrm{C}$ erst bei 34 parallelen Zellen erreicht, was einem Energieinhalt von $457 \mathrm{kWh}$ entspricht. Der entsprechende SOC-Verlauf ist in Abbildung 5-16 gezeigt. Zur Bewältigung der täglichen Fahraufgabe bei $-12,6^{\circ} \mathrm{C}$ müsste die Batterie gegenüber dem Basisszenario mit 10 parallelen Zellen und $135 \mathrm{kWh}$ Energieinhalt um 
das 3,4-fache überdimensioniert werden. Eine mögliche Option zur Vermeidung dieser Überdimensionierung stellt der Einsatz einer Batterieheizung dar, welche in Kapitel 5.1.6 behandelt wird.

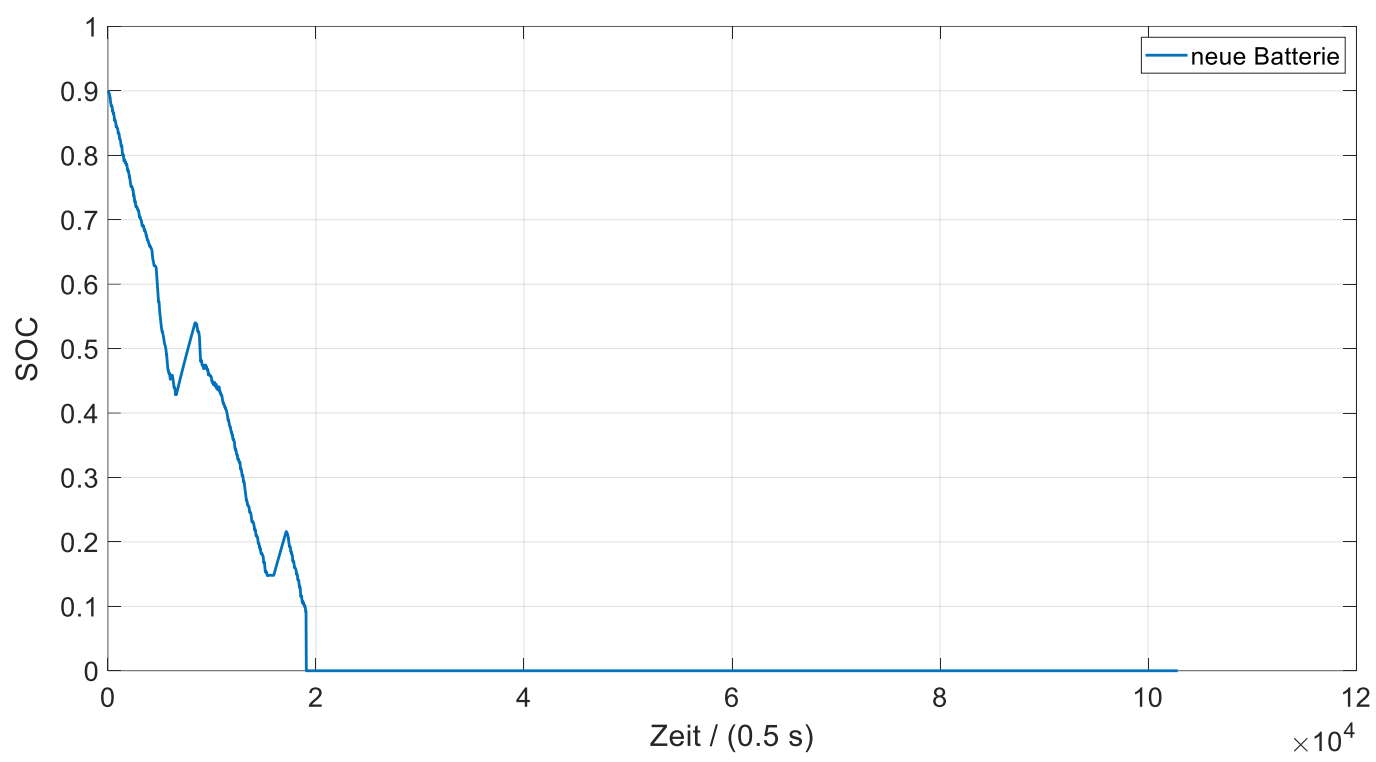

Abbildung 5-15: SOC-Verlauf der Basisszenario-Konfiguration bei $-12.6^{\circ} \mathrm{C}(10$ parallele Zellen, $135 \mathrm{kWh})$

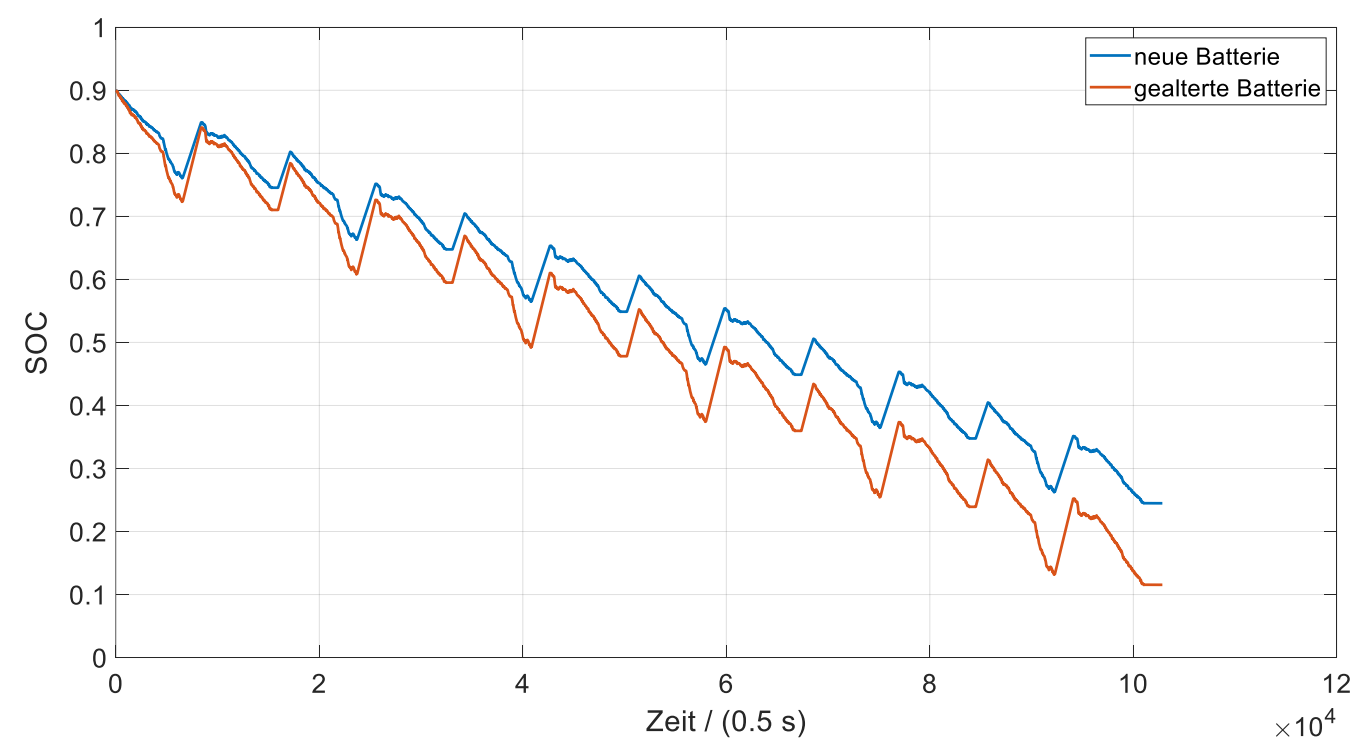

Abbildung 5-16: SOC-Verlauf mit 34 parallelen Zellen (457 kWh) bei $-12.6^{\circ} \mathrm{C}$ 
Die nun folgenden Untersuchungen zur Lebensdauer bei verschiedenen Granularitätsstufen der Temperaturverteilung basieren auf einer Batterie mit $457 \mathrm{kWh}$ Energieinhalt. Die Ergebnisse der Lebensdauerberechnungen sind in Tabelle 5-7 gegeben und in Abbildung 5-17 veranschaulicht.

\section{Tabelle 5-7: Auswirkung der Granularitätsstufen der Temperaturverteilung auf die Lebensdauer}

\begin{tabular}{ccc}
\hline $\begin{array}{c}\text { Granularität der } \\
\text { Temperaturverteilung }\end{array}$ & \multicolumn{2}{c}{ Lebensdauer } \\
\hline $25^{\circ} \mathrm{C}$ konstant & 3687 Tage & $86,4 \%$ \\
$15^{\circ} \mathrm{C}$ konstant & 4195 Tage & $98,3 \%$ \\
$10^{\circ} \mathrm{C}$ konstant & 4330 Tage & $101,4 \%$ \\
Monatsmittelwerte & 4305 Tage & $100,8 \%$ \\
Tagesmittelwerte & 4269 Tage & $100 \%$ \\
\hline
\end{tabular}

Für die folgende Auswertung wird die Rechnung mit Tagesmittelwerten als Referenz herangezogen, da dies die feinste Granularitätsstufe darstellt. Ein unmittelbarer Vergleich mit dem Basisszenario ist nicht möglich, da den Simulationen eine größere Batterie zugrunde liegt und die elektro-thermischen Belastungen und damit auch die Alterungsberechnungen nicht vergleichbar sind.

In Tabelle 5-7 und Abbildung 5-17 ist erkennbar, dass bei Verwendung von Monatsmittelwerten die Lebensdauer gegenüber Tagesmittelwerten (Referenz) um 0,8\% und damit nur in sehr geringem Maße überschätzt wird. Bei Verwendung einer konstanten Umgebungstemperatur von $10^{\circ} \mathrm{C}$ wird die Lebensdauer um $1,4 \%$ überschätzt. Bei einer weiteren Erhöhung der Umgebungstemperatur stellt sich hingegen ein anderer Zusammenhang ein. Mit steigenden Werten wird die Lebensdauer zunehmend unterschätzt. Während bei $15^{\circ} \mathrm{C}$ die Abweichung mit $1,7 \%$ noch vergleichsweise moderat ausfällt, wurde bei $25^{\circ} \mathrm{C}$ mit $13,6 \%$ bereits eine deutliche Abweichung ermittelt. Dieser überproportionale Anstieg kann auf die 10-K-Regel zurückgeführt werden (Vgl. Kapitel 4.1.5), wonach höhere Temperaturen zu einer beschleunigten Alterung führen.

Es kann geschlussfolgert werden, dass für die Alterungsberechnung feine Granularitäten der Temperaturverteilung nur einen geringen Zugewinn hinsichtlich der Ergebnisgenauigkeit liefern. Die Betrachtung mit einer konstanten Umgebungstemperatur ist dann zulässig, wenn diese möglichst nah am eigentlichen Mittelwert der Temperatur des betrachteten Ortes liegt. Für die Ermittlung der Batterieperformance ist es jedoch zwingend erforderlich, dass die elektro-thermische Belastung und die Leistungsfähigkeit der Batterie bei niedrigen Umgebungstemperaturen überprüft 
werden. Im hier dargestellten Beispiel kann das betrachtete Fahrszenario aufgrund der Temperaturabhängigkeit der Batteriekapazität nur mit einem sehr großen Energieinhalt bewältigt werden. Die Berechnung auf Basis einer konstanten Temperatur würde demnach zu falschen Aussagen zur technischen Machbarkeit (vor allem an sehr kalten Tagen) führen.

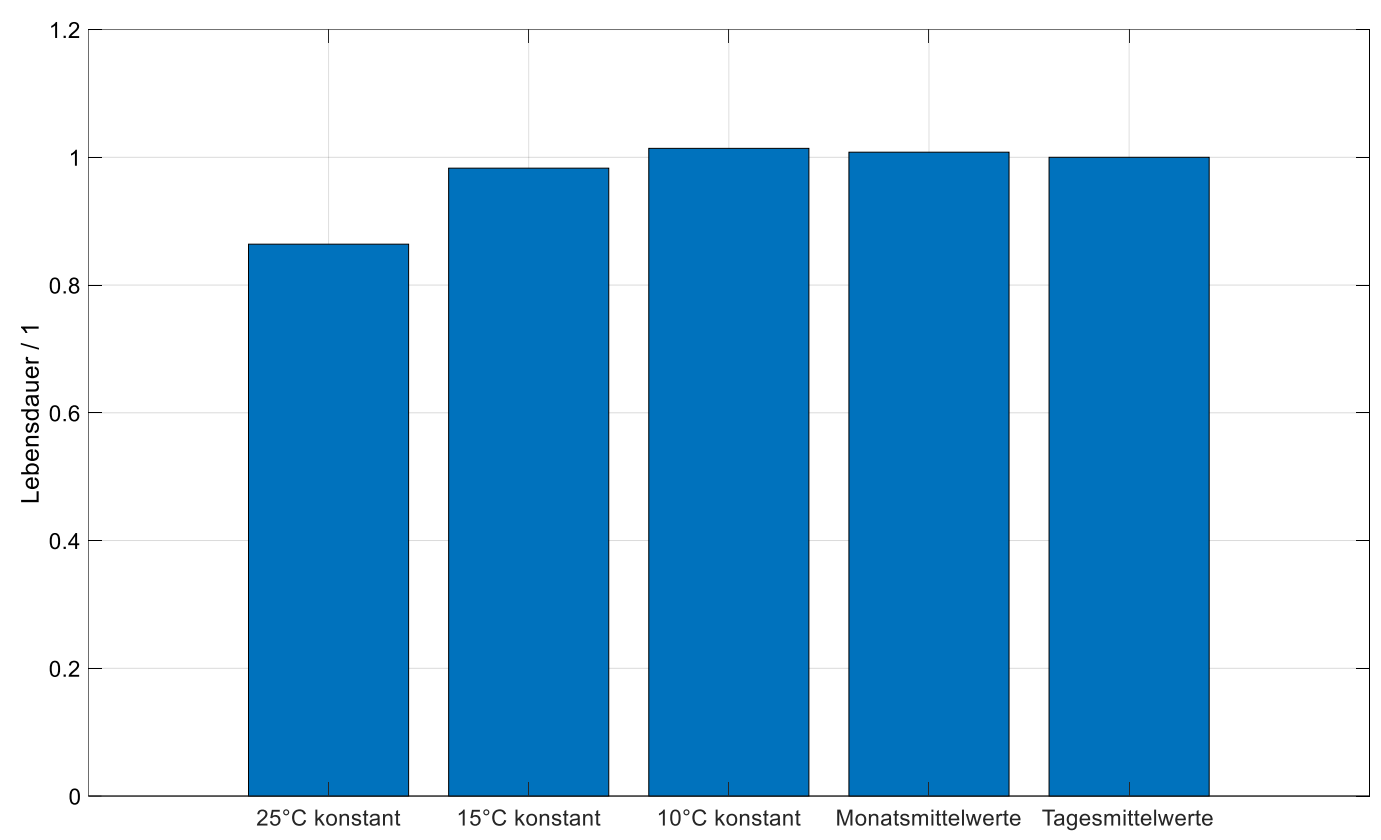

\section{Abbildung 5-17: Batterielebensdauer bei verschiedenen Granularitätsstufen der Temperaturverteilung}

Zusammenfassend kann festgehalten werden, dass hohe Temperaturen die Thematik der Batteriealterung betreffen, während niedrige Temperaturen die Frage der Batterieperformance stellen. Demzufolge ist die technische Ausrüstung der Fahrzeuge stets an den jeweiligen Einsatzort anzupassen. Ein Betrieb in nördlichen Ländern wie beispielsweise Skandinavien stellt andere Anforderungen an die Batterie als ein Betrieb in Südeuropa.

\subsubsection{Fahrzeugbetrieb mit Batterieheizung}

Aus Abbildung 5-15 in Kapitel 5.1.5 geht hervor, dass der Energieinhalt und die Ladeleistung des Basisszenarios bei tiefen Temperaturen unter $0^{\circ} \mathrm{C}$ nicht ausreichend sind, um die tägliche Fahrleistung zu erbringen. Für sehr kalte Tage muss die Batterie demnach teilweise deutlich überdimensioniert werden, um die Temperaturabhängigkeit der Batteriekapazität (Vgl. Abbildung 4-6) zu kompensieren. Dadurch wird die Traktionsbatterie sehr schwer und wirkt sich entsprechend negativ auf die zulässige Anzahl an 
Fahrgästen sowie die Energiebilanz des Fahrzeugs und des Gesamtsystems aus. Viele Fahrzeughersteller bieten deshalb neben einer Kühlung auch eine Batterieheizung an. Damit kann einerseits eine reduzierte Kapazität bei niedrigen Temperaturen kompensiert werden, andererseits muss die benötigte Heizenergie aus der Traktionsbatterie bereitgestellt werden und steht somit nicht mehr für die Fahraufgabe zur Verfügung, was wiederum zu Lasten der täglichen Reichweite geht.

In Rahmen dieser Arbeit werden ein einfaches Batterieheizungsmodell und ein Regler mit Temperaturhysterese implementiert. Als Eingangsparameter sind die Batteriesolltemperatur $T_{\text {Batt,soll }}$ und die Heizleistung festgelegt sowie eine Hysteresetemperatur $T_{\text {Batt,Hysterese }}$ definiert. Die Regelstrategie sieht vor, dass die Heizung dann aktiviert wird, wenn die Batterietemperatur einen Schwellwert unterschreitet und so lange aktiviert bleibt, bis die Solltemperatur erreicht wird. Der Schwellwert berechnet sich aus der Differenz des Sollwerts und der Hysterese. Die Bedingung zur Aktivierung der Batterieheizung ist wie folgt definiert.

$$
T_{\text {Batt }}\left(t_{1}\right)<T_{\text {Batt,soll }}-T_{\text {Batt,Hysterese }}
$$
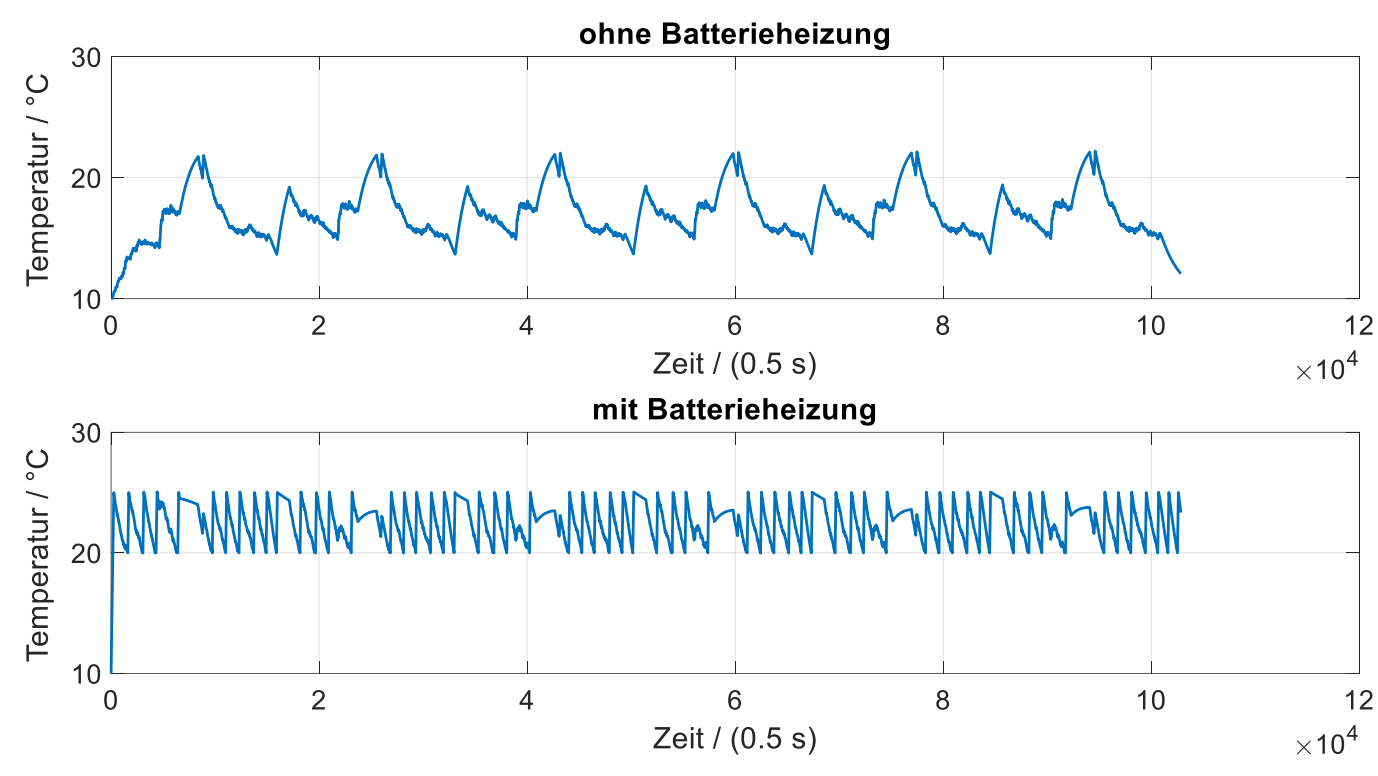

\section{Abbildung 5-18: Batterietemperaturverlauf des Basisszenarios, oben: ohne Batterieheizung, unten: mit Batterieheizung}

Im Rahmen dieser Arbeit wird angenommen, dass die Heizleistung direkt und verlustfrei in die Batterie eingeprägt wird. In einer praktischen Ausführung würde dies beispielsweise einer Heizfolie entsprechen, welche zwischen den Zellen oder am Boden der Zellen angebracht wird. Eine Integration in den Kühlkreislauf der Batterie ist jedoch 
ebenso möglich. Übliche Leistungen für Batterieheizsysteme liegen bei ca. 5 kW (Hipp, 2019).

Abbildung 5-18 zeigt einen Temperaturverlauf des Basisszenarios (bei $10^{\circ} \mathrm{C} \mathrm{Um-}$ gebungstemperatur) mit und ohne Batterieheizung. In diesem Beispiel wurde die Heizleistung mit $5 \mathrm{~kW}$ parametriert, die Solltemperatur auf $25^{\circ} \mathrm{C}$ festgelegt und eine Hysterese von $5^{\circ} \mathrm{C}$ gewählt. Der Schwellwert beträgt somit $20^{\circ} \mathrm{C}$. Aus der Abbildung geht hervor, dass die Charakteristik des Verlaufs bei Verwendung des Batterieheizungsreglers eine vollständig andere ist als bei ausgeschalteter Heizung. Es ergibt sich ein nahezu konstantes Temperaturband. Dadurch kann eine Reduzierung der Kapazität durch niedrige Temperaturen und folglich eine Überdimensionierung der Batterie vermieden werden.

\subsection{Detailuntersuchungen zur Alterung der Traktionsbatterie}

In Kapitel 5.1 wurden verschiedene Einsatzszenarien definiert und hinsichtlich ihrer Auswirkungen auf die Lebensdauer der Batterie untersucht. Dabei standen vor allem operative Betriebsabläufe des ÖPNV im Vordergrund. In diesem Abschnitt werden nun verschiedene Granularitäten und Abhängigkeiten der Alterungsberechnung betrachtet.

\subsubsection{Sensitivitätsanalyse der Makrozeitschrittweite}

Gemäß Abbildung 3-1 findet die Batteriesimulation auf zwei unterschiedlichen Zeitschrittebenen statt. In diesem Abschnitt wird eine Variation der Makrozeitschrittweite $\Delta \tau_{\text {makro }}$ vorgenommen und die Auswirkungen auf die Ergebnisgenauigkeit der Alterungsberechnung untersucht. In den bisherigen Betrachtungen in Kapitel 5.1 wurde stets eine Schrittweite von $\Delta \tau_{\text {makro }}=1 \mathrm{~d}$ verwendet. Damit verbunden ist jedoch eine große Rechendauer, da je nach resultierender Lebensdauer die Simulationsschleife nach Abbildung 3-1 sehr oft durchlaufen werden muss.

Es wird zunächst für das Basisszenario eine Variation der Makrozeitschrittweite $\Delta \tau_{\text {makro }}$ vorgenommen. Die Simulationsergebnisse sind in Tabelle 5-8 dargestellt und in Abbildung 5-19 veranschaulicht. Daraus geht hervor, dass eine Erhöhung der Makrozeitschrittweite $\Delta \tau_{\text {makro }}$ nur zu vergleichsweise geringen Änderungen der resultierenden Lebensdauer führt. Die steigende Schrittweite bedingt eine Verlängerung und damit Überschätzung der Batterielebensdauer. Dies ist auf den selbstverstärkenden Effekt der Alterung bei Belastung mit einem konstanten Leistungsprofil zurückzuführen, welcher in Kapitel 5.1.2 erläutert (Vgl. Abbildung 5-7 und Abbildung 5-8) wurde. 
Tabelle 5-8: Sensitivität der Lebensdauer in Abhängigkeit der Makrozeitschrittweite (Basisszenario)

\begin{tabular}{ccc}
\hline $\begin{array}{c}\text { Makrozeitschrittweite } \\
\Delta \boldsymbol{\tau}_{\text {makro }}(\mathbf{d})\end{array}$ & \multicolumn{2}{c}{ Lebensdauer } \\
\hline 1 & 1636 Tage & $100,00 \%$ \\
10 & 1636 Tage & $100,00 \%$ \\
100 & 1640 Tage & $100,24 \%$ \\
1000 & 1670 Tage & $102,08 \%$ \\
2000 & 1698 Tage & $103,79 \%$ \\
\hline
\end{tabular}

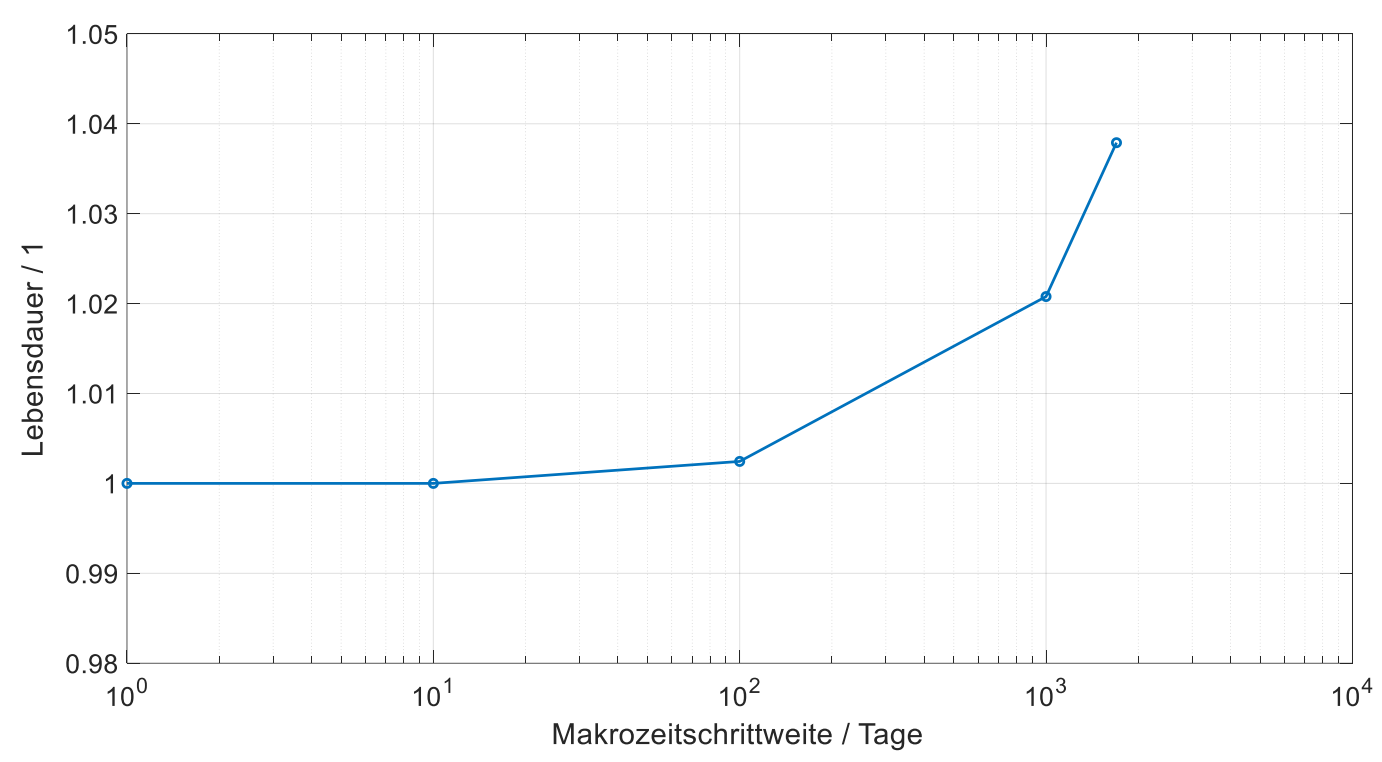

Abbildung 5-19: Sensitivität der Ergebnisgenauigkeit der Alterungsberechnung in Abhängigkeit der Makrozeitschrittweite (Basisszenario)

Eine Änderung der Schrittweite von $\Delta \tau_{\text {makro }}=1 d$ auf $\Delta \tau_{\text {makro }}=10 \mathrm{~d}$ bewirkt noch keine Überschätzung der Batterielebensdauer. Eine weitere Erhöhung der Schrittweite auf $\Delta \tau_{\text {makro }}=100 d$ führt nur zu einer um 0,24\% längeren Lebensdauer. Gleichzeitig gilt es zu beachten, dass eine Erhöhung der Makrozeitschrittweite einer proportionalen Reduzierung der benötigten Rechendauer entspricht. Bei einer Makrozeitschrittweite von $\Delta \tau_{\text {makro }}=2000 d$ wird folgerichtig nur ein Zyklus gemäß Abbildung 3-1 durchlaufen. Das bedeutet, dass auf Basis der elektrothermischen Simulation die resultierende Alterung nur einmal bestimmt und diese anschließend linear extrapoliert wird. Bei allen anderen Schrittweiten wird die elektrothermische Simulation entsprechend 
öfter durchgeführt und die resultierende Alterung zwischen den Zeitschritten extrapoliert.

Aus den Ergebnissen kann geschlussfolgert werden, dass durch eine erhebliche Reduzierung der Rechendauer, bedingt durch große Zeitschrittweiten, nur ein vergleichsweise kleiner methodischer Fehler in der Alterungsberechnung entsteht.

Im Folgenden wird der Untersuchungsraum um die Dimension des SOC-Hubs erweitert, um zusätzlich dessen Einfluss auf die Ergebnisgenauigkeit zu ermitteln. Die Variation des SOC-Hubs wird nur mittels unterschiedlicher Ladeleistungen realisiert, sodass eine möglichst gute Vergleichbarkeit der einzelnen Berechnungen möglich ist. Dabei bewirkt eine höhere Ladeleistung einen kleineren SOC-Hub, wobei der Zusammenhang nichtlinear ist, wie die Ergebnisse in Tabelle 5-9 zeigen. Die Systemkonfiguration des Basisszenarios hat bereits einen maximalen SOC-Hub von $\triangle S O C=0,37$ bei einer neuen Batterie (Abbildung 5-6). Für eine möglichst breite Variation des SOCHubs wird deshalb eine größere Batteriekonfiguration mit 21 parallelen Zellen (283 kWh) gewählt. Die Ergebnisse der Simulationen sind in Tabelle 5-9 dargestellt und in Abbildung 5-20 veranschaulicht.

Tabelle 5-9: Sensitivität der Lebensdauer (in Tagen) in Abhängigkeit der Makrozeitschrittweite (Variation der Zeitschrittweite und des SOC-Hubs)

\begin{tabular}{|c|c|c|c|c|c|c|c|c|c|}
\hline \multirow{2}{*}{\multicolumn{3}{|c|}{$\begin{array}{l}\text { Ladeleistung }(\mathrm{kW}) \\
\qquad \mathrm{SOC}\end{array}$}} & 140 & 160 & 180 & 200 & 220 & 240 & 260 \\
\hline & & & 0,581 & 0,427 & 0,275 & 0,174 & 0,170 & 0,170 & 0,170 \\
\hline \multirow{5}{*}{ 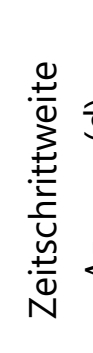 } & \multirow{5}{*}{ 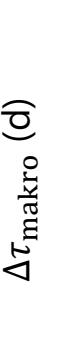 } & 1 & 2322 & 2841 & 2970 & 2991 & 3004 & 3017 & 3031 \\
\hline & & 10 & 2324 & 2841 & 2970 & 2991 & 3004 & 3017 & 3031 \\
\hline & & 100 & 2337 & 2845 & 2971 & 2991 & 3005 & 3018 & 3032 \\
\hline & & 1000 & 2438 & 2873 & 2979 & 2997 & 3011 & 3024 & 3037 \\
\hline & & 4000 & 2623 & 2925 & 2995 & 3009 & 3022 & 3035 & 3049 \\
\hline
\end{tabular}

Bei Auswertung der Tabelle 5-9 und Abbildung 5-20 wird die grundlegende Aussage des Basisszenarios bestätigt, wonach mit steigender Makrozeitschrittweite die Lebensdauer der Batterie überschätzt wird. Zusätzlich ist jedoch erkennbar, dass die Höhe der Überschätzung nicht konstant ist, sondern auch vom SOC-Hub abhängt und mit steigendem Hub überproportional zunimmt. Bis zu einer Schrittweite von $\Delta \tau_{\text {makro }}=100 \mathrm{~d}$ liegen die Kurven für alle SOC-Hübe deutlich unterhalb von 1\% Abweichung, bezogen auf den Wert $\Delta \tau_{\text {makro }}=1$. Zwischen den Schrittweiten $\Delta \tau_{\text {makro }}=100 d$ und $\Delta \tau_{\text {makro }}=$ $1000 d$ vergrößert sich die Abweichung der Kurven auf sehr unterschiedliche Art und Weise. Die Kurve mit dem größten SOC-Hub $(0,58)$ weist dabei den größten Anstieg 
und somit die größte Abweichung auf. Bei $\Delta \tau_{\text {makro }}=1000 d$ wird die Lebensdauer bereits um $5 \%$ überschätzt.

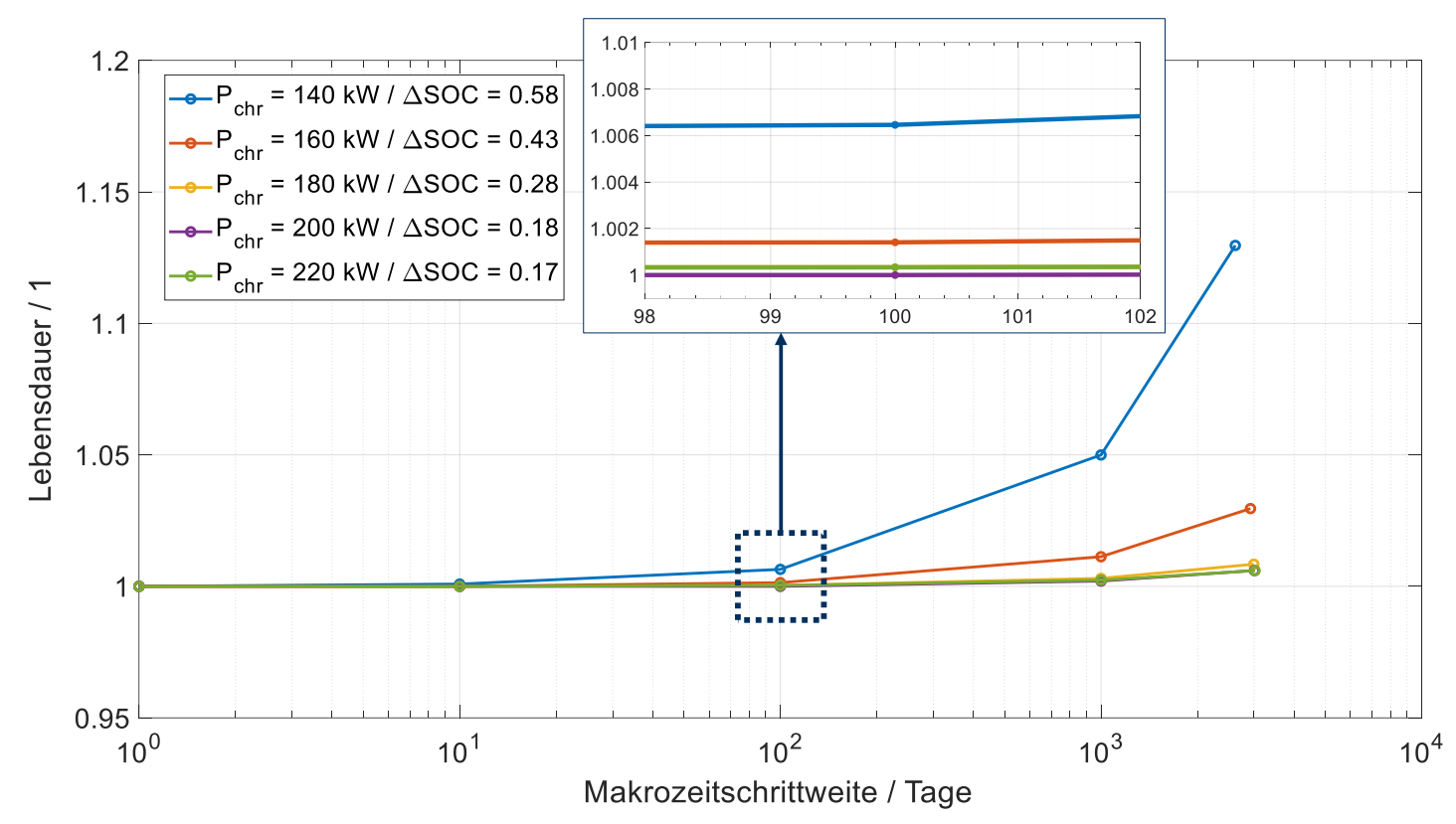

\section{Abbildung 5-20: Sensitivität der Lebensdauer in Abhängigkeit der Makrozeitschrittweite, (Variation der Zeitschrittweite und des SOC-Hubs)}

Aus den Ergebnissen der Berechnungen kann geschlussfolgert werden, dass Makrozeitschrittweiten bis $\Delta \tau_{\text {makro }}=100 \mathrm{~d}$ nur zu einem sehr geringen Fehler in der Alterungsberechnung führen und somit für die Systemsimulation anwendbar sind. Vor allem für Optimierungsrechnungen, in denen viele Systemkonfigurationen berechnet werden sollen, ist eine Verwendung der Schrittweite von $\Delta \tau_{\text {makro }}>1 d$ aufgrund der überproportional sinkenden Rechendauer empfehlenswert.

\subsubsection{Sensitivitätsanalyse der Schädigungsfaktoren}

In diesem Abschnitt werden die einzelnen Schädigungsfaktoren des Alterungsmodells detailliert analysiert. Für die Untersuchungen wird die Konfiguration des Basisszenarios verwendet. Die Simulation wird bei Makrozeitschrittweite $\Delta \tau_{\text {makro }}=1 d$ durchgeführt. Die Schädigungsfaktoren liegen in jedem Makrozeitschritt für die in der Zyklusanalyse erstellten alterungsäquivalenten Teilzyklen vor. Diese Teilzyklen unterscheiden sich jedoch in unterschiedlichem Maße, je nach voranschreitender Alterung. Sie sind deshalb nicht unmittelbar miteinander vergleichbar. Es wird deshalb ein resultierender Wert jedes Schädigungsfaktors für jeweils einen Betriebstag (entspricht einem Makrozeitschritt) ermittelt. Dies wird über eine Wichtung des Ladungsdurchsatzes erreicht. 
Für jeden äquivalenten Teilzyklus liegt die anteilig durchgesetzte Ladungsmenge vor, ${ }^{16}$ welche in die Berechnung der Alterung gemäß den Formeln (4-31) und (4-32) eingeht. Die berechneten Alterungsfaktoren werden mit dem gewichteten Ladungsdurchsatz multipliziert und anschließend aufsummiert. Für einen Betriebstag liegen nun 4 Alterungsfaktoren (jeweils für Kapazität und Innenwiderstand) vor, welche mit anderen Tagen verglichen werden können.

Abbildung 5-21 zeigt die gewichteten Schädigungsfaktoren über den Makrozeitschritten (Absolutwerte). In Abbildung 5-22 sind die Verläufe zugunsten einer besseren Vergleichbarkeit um den jeweiligen Startwert $S\left(\tau_{\text {makro }}=1 d\right)$ vermindert dargestellt. Beide Abbildungen bestätigen die in Abbildung 5-8 dargestellten Zusammenhänge, wonach die Batteriealterung in Elektrobussen aufgrund der konstanten Leistungsanforderung ein sich selbstverstärkender Effekt ist.
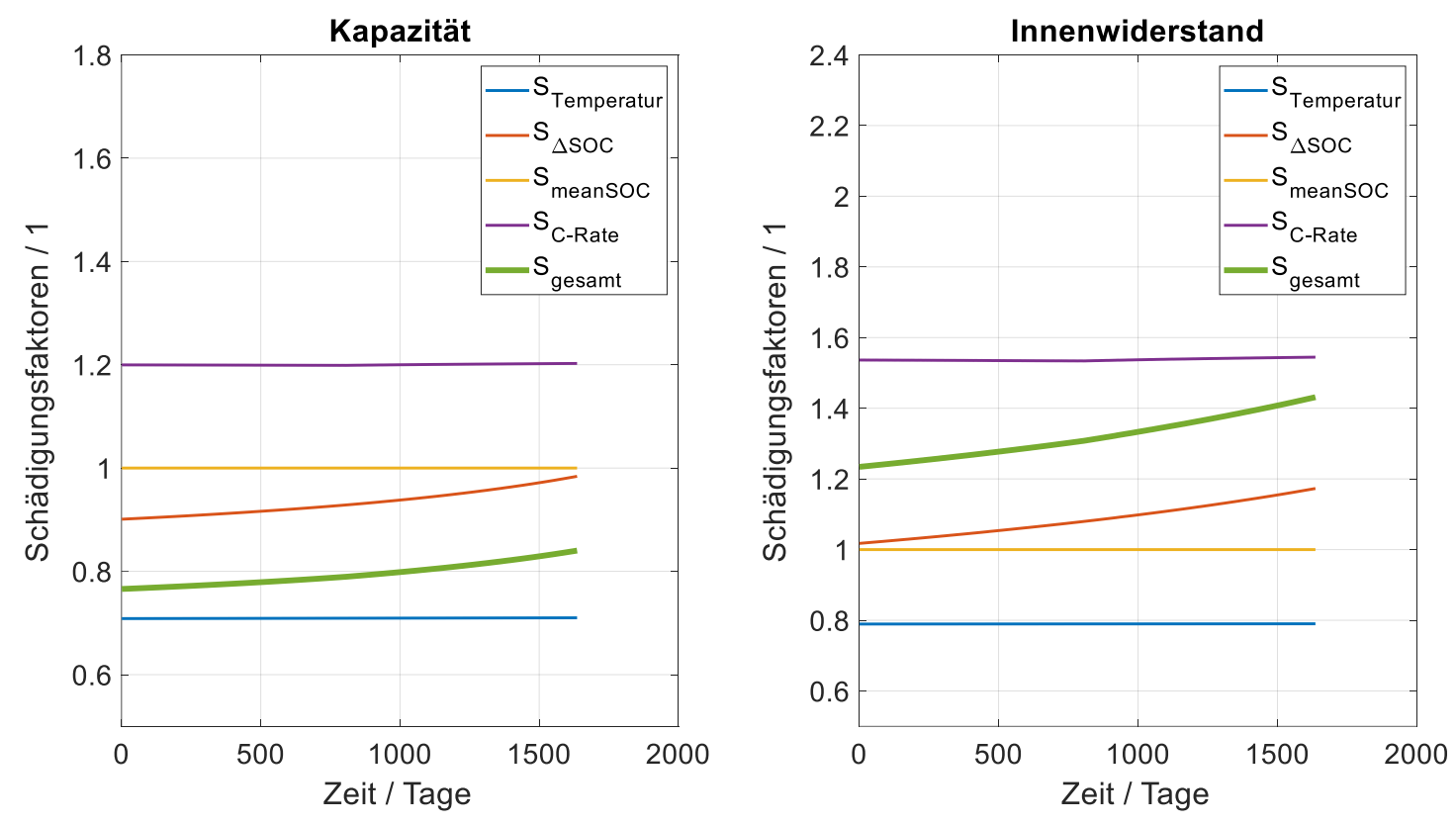

Abbildung 5-21: Verlauf der Alterungsfaktoren über Makrozeitschritten

Zunächst ist in Abbildung 5-21 erkennbar, dass die Temperatur den geringsten Anteil an der Gesamtalterung hat. Dies ist mit der vergleichsweise niedrigen Umgebungstemperatur von $15^{\circ} \mathrm{C}$ zu begründen. Bei höheren Temperaturen steigt der Schädigungsanteil gemäß der ermittelten Schädigungsfunktion (Vgl. Abbildung 4-26) überproportional an. Für die Kapazität beträgt der Schädigungsfaktor ca. 0,75 und für den Innenwiderstand 0,80. Es ist erkennbar, dass beide über dem betrachteten Zeitraum nahezu konstant bleiben. Bei Annahme einer konstanten Umgebungstemperatur über

\footnotetext{
${ }^{16}$ Die jeweils durchgesetzte Ladungsmenge der einzelnen Teilzyklen wird in der Zyklusanalyse unter Verwendung des Rainflow-Zählverfahrens berechnet (Vgl. Abbildung 4-32).
} 
dem Berechnungszeitraum findet demnach trotz steigendem Innenwiderstand keine alterungsrelevante Erhöhung der Batterietemperatur statt.

Die C-Rate hat im Basisszenario den betragsmäßig größten Einfluss auf die Alterung. Der Schädigungsfaktor für die Kapazität beträgt 1,2 und bleibt über dem Betrachtungszeitraum nahezu konstant. Für den Innenwiderstand beträgt dieser ca. 1,55. Dies ist vor allem auf den Umstand des negativen Anstiegs der Schädigungsfunktion zurückzuführen (Vgl. Kapitel 4.1.5, Abbildung 4-29). Im Basisszenario werden mit ca. 1C, sowohl beim Laden als auch beim Entladen, vergleichsweise kleine Stromraten erreicht, wodurch der resultierende Schädigungsfaktor aufgrund des negativen Anstiegs der Funktion entsprechend hoch ist.
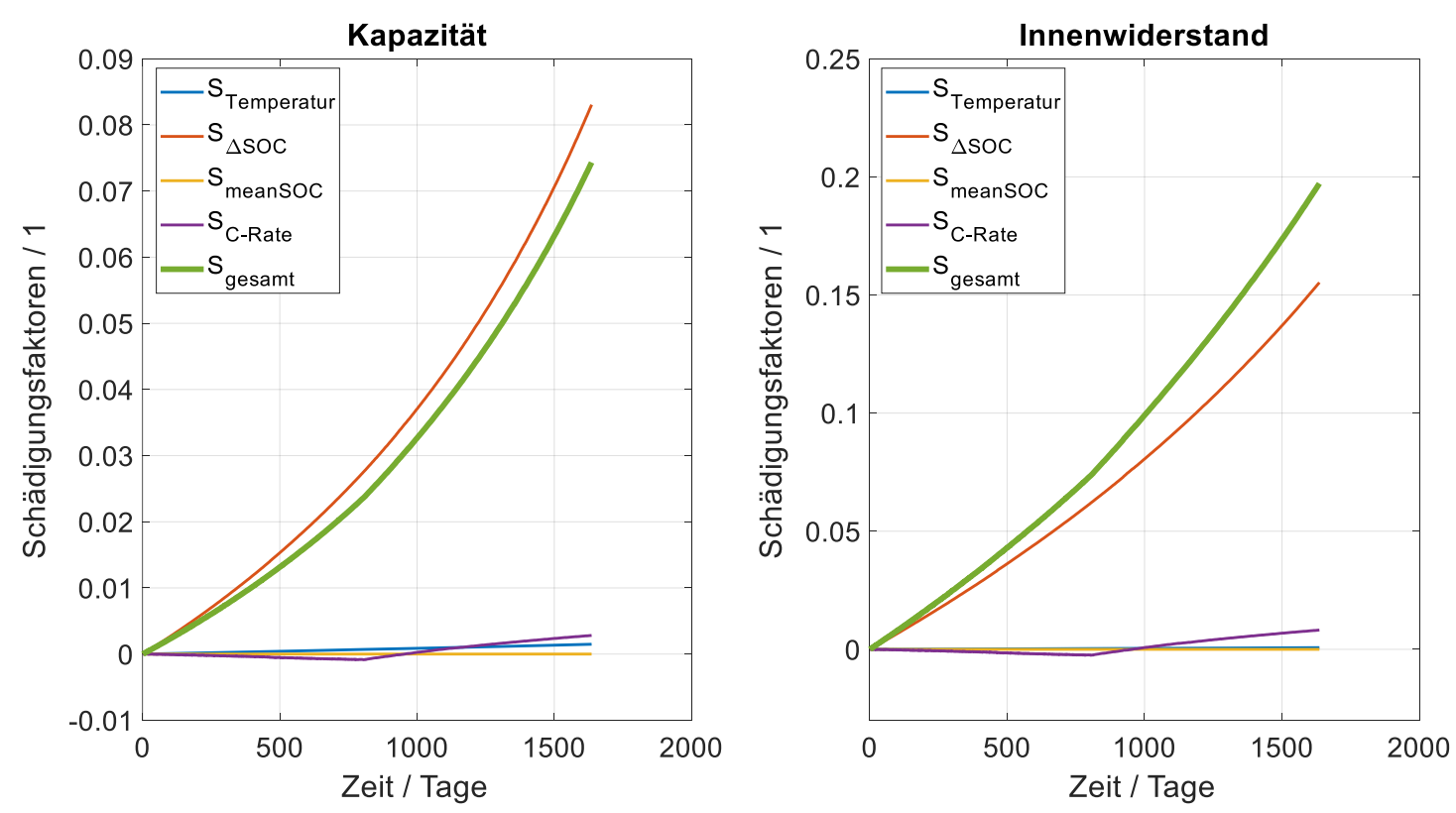

Abbildung 5-22: Änderung der Schädigungsfaktoren über Makrozeitschritten

Die Schädigung durch den SOC-Hub ( $\mathbf{\Delta} \mathbf{S O C}$ ) weist über dem Betrachtungszeitraum sowohl für die Kapazität als auch für den Innenwiderstand einen exponentiellen Verlauf auf. Aus den Abbildungen geht hervor, dass der qualitative Verlauf der Gesamtalterung maßgeblich durch die Schädigung des SOC-Hubs beeinflusst wird. Für die Kapazität startet der Schädigungsfaktor bei 0,9 und steigt bis auf 1,0 an. Für den Innenwiderstand liegt der Anfangswert des Schädigungsfaktors bereits über 1,0 und steigt auf nahezu 1,2 an. Da der SOC-Hub eine der wesentlichen Beurteilungsgrößen der Speicherbetriebsstrategie ist, kann mittels dessen Steuerung der Verlauf der Batteriealterung maßgeblich beeinflusst werden.

Der mittlere SOC ist für die zyklische Alterung nicht parametriert und ist deshalb gleich 1 gesetzt. 


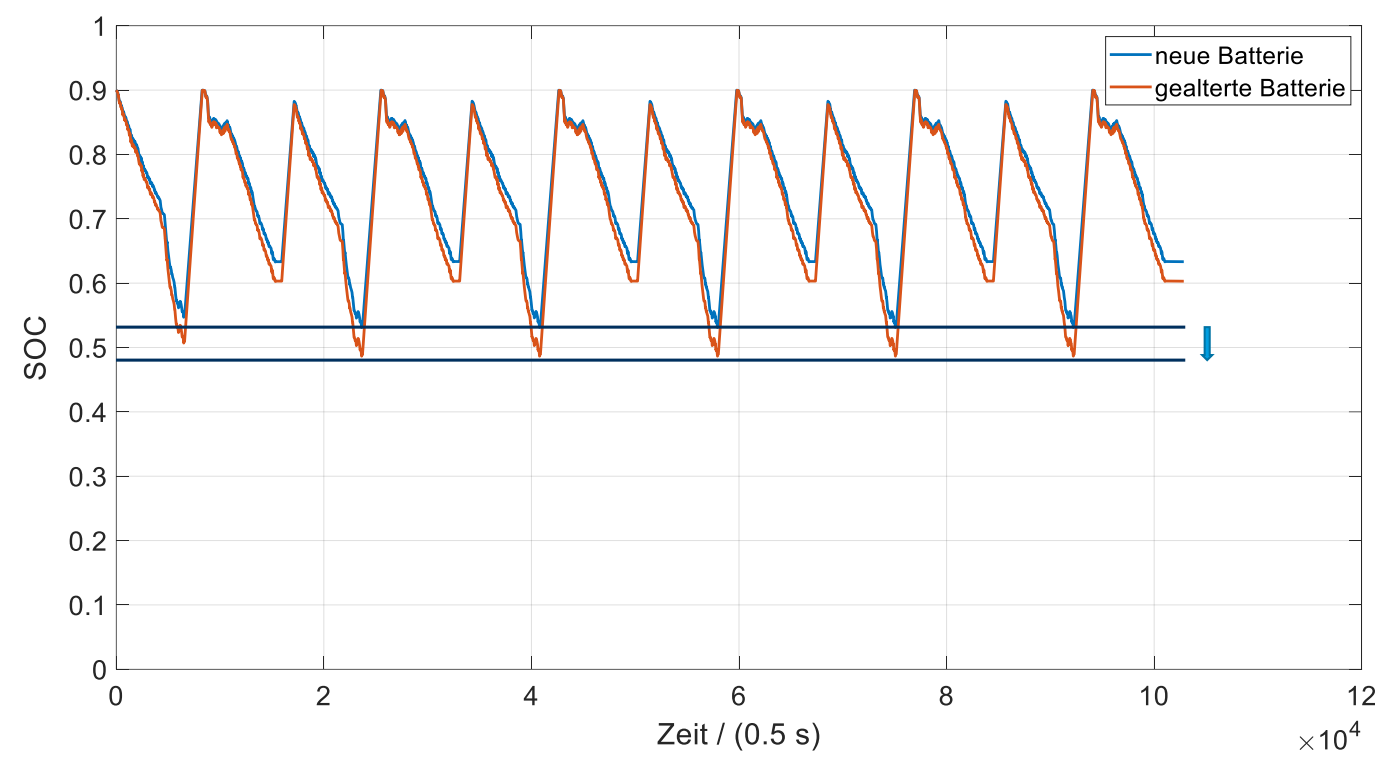

Abbildung 5-23: Vergrößerung des SOC-Hubs durch Alterung (Basisszenario bis

$$
\left.\tau_{\text {makro }}=\mathbf{8 1 0} d\right)
$$

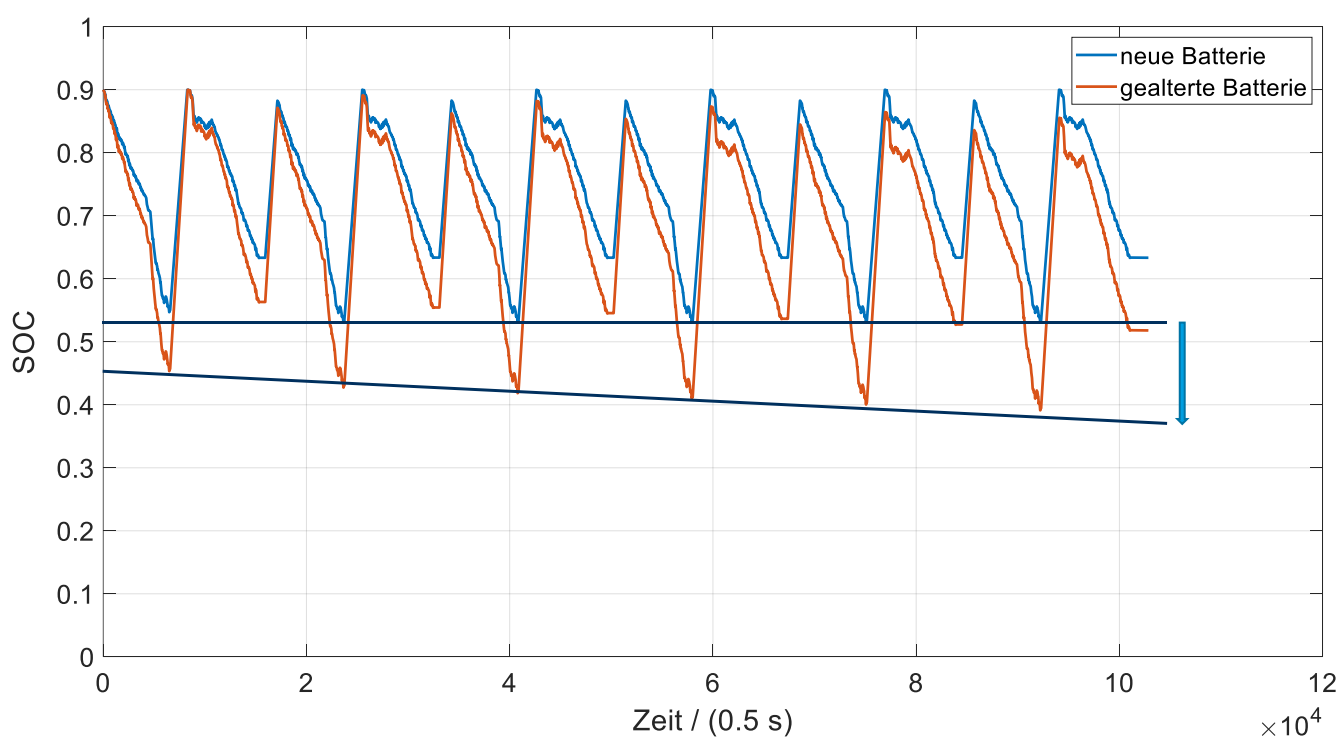

Abbildung 5-24: Vergrößerung des SOC-Hubs durch Alterung und unzureichende Nachladung (Basisszenario von $\tau_{\text {makro }}=810 d$ bis $\tau_{\text {makro }}=1636 d$ )

Aufgrund der Abnahme der Kapazität sowie der sich ständig vergrößernden elektrothermischen Belastung (bedingt durch eine schneller einbrechende Ruhespannung und 
den Anstieg des Innenwiderstands) steigt der SOC-Hub im Laufe der Zeit bei konstanter Leistungsanforderung an. Die Geschwindigkeit des Anstiegs wird zum einen von der Abnahme der Kapazität beeinflusst. Diese Beeinflussung ist immer vorhanden, unabhängig von der jeweils gewählten Systemkonfiguration. Die Vergrößerung des SOC-Hubs kann dabei als eine Art Parallelverschiebung des minimalen SOCs nach unten aufgegriffen werden, was in Abbildung 5-23 verdeutlicht ist. Es handelt sich um die Darstellung des SOC-Verlaufs im Basisszenario bei Makrozeitschritt $\tau_{\text {makro }}=810 d$. Zum anderen kann jedoch ein zweiter Effekt zur Beeinflussung des SOC-Hub-Anstiegs hinzukommen. Wenn der SOC-Verlauf über den Tag nicht ladungserhaltend ist, so vergrößert sich der SOC-Hub analog. Eine solche Speicherbetriebsstrategie kann bereits mit einer neuen Batterie aktiv gewählt werden und verstärkt sich im Laufe des Batterielebens ${ }^{17}$. Es ist jedoch auch möglich, dass dieser Effekt erst im Laufe der Zeit auftritt, wie dies beispielsweise im Basisszenario der Fall ist. Ab Makrozeitschritt $\tau_{\text {makro }}>810 d$ steigt der SOC-Hub neben der alterungsbedingten Abnahme der Kapazität um einen zusätzlichen Anteil an, wie mittels des SOC-Verlaufs in Abbildung 5-24 verdeutlicht wird. Die Ladeleistung ist nun nicht mehr ausreichend, um die Batterie auf den festgelegten Wert $\mathrm{SOC}_{\max }$ zu laden. Somit ergibt sich ein SOC-Verlauf, welcher nicht mehr ladungserhaltend ist und damit eine zusätzliche Beschleunigung der Zunahme des SOC-Hubs.
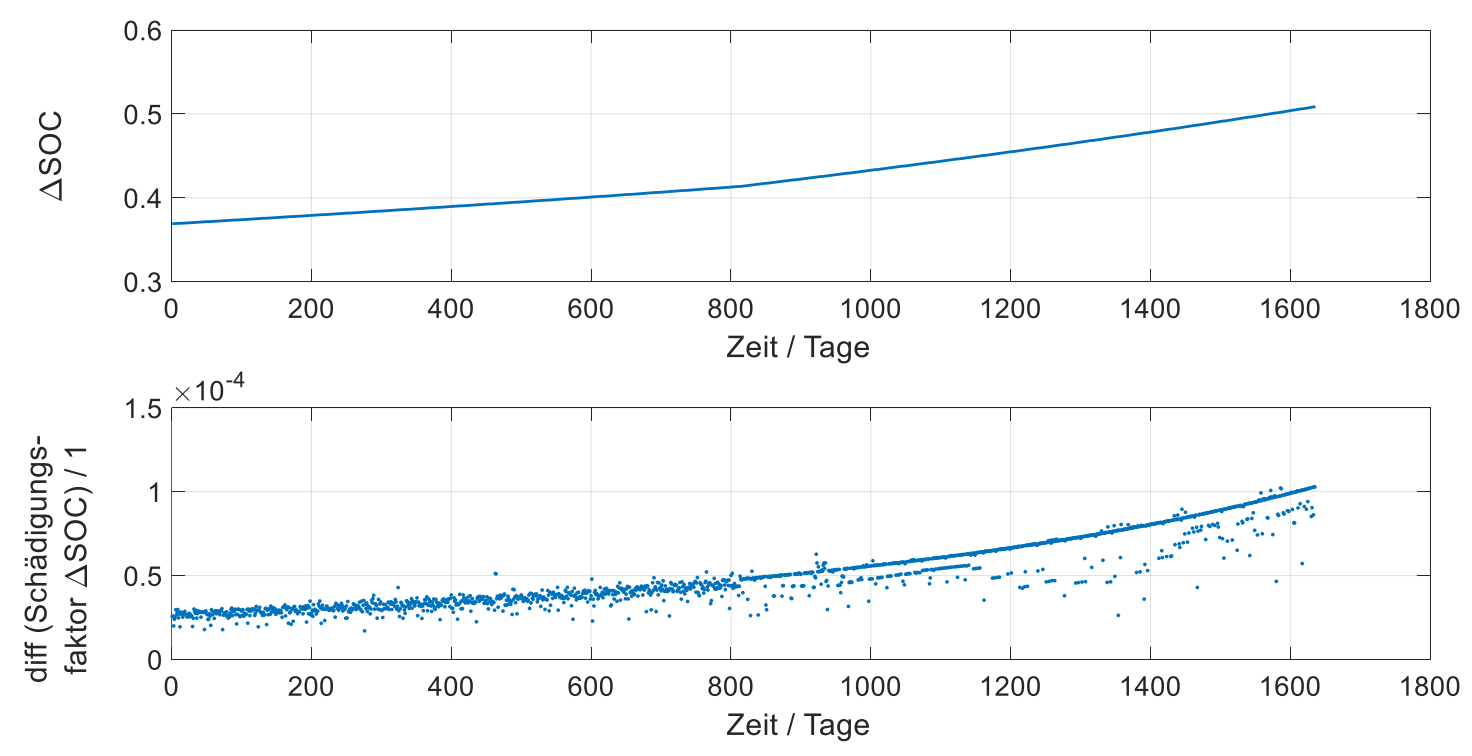

\section{Abbildung 5-25: Detailanalyse SOC-Hub: oben: Zunahme SOC-Hub über Makrozeitschritten, unten: differenzierter Verlauf des Schädigungsfaktors (Kapazität)}

\footnotetext{
${ }^{17}$ Eine solche Speicherbetriebsstrategie mit einem nicht-ladungserhaltenden SOC-Verlauf bei neuer und gealterter Batterie ist beispielsweise in Abbildung 5-16 gegeben.
} 
In Abbildung 5-25 (oben) ist der jeweils maximale SOC-Hub über den Makrozeitschritten abgebildet. Daraus geht hervor, dass die Funktion bei $\tau_{\text {makro }}=810 d$ eine Änderung im Anstiegsverhalten aufweist. Dieser Zusammenhang zeigt sich auch im resultierenden Schädigungsfaktor der Kapazität, welcher in numerisch differenzierter Form in Abbildung 5-25 (unten) dargestellt ist. Es ist eine deutliche Änderung im Verhalten vor und nach dem Zeitpunkt $\tau_{\text {makro }}=810 \mathrm{~d}$ erkennbar. Während die Werte im Bereich $\tau_{\text {makro }} \leq 810 d$ einen moderaten Anstieg und eine gleichmäßige Streuung aufweisen ${ }^{18}$, ergibt sich für $\tau_{\text {makro }}>810 d$ ein deutlich markanterer Verlauf mit steilerem Anstieg und einer ungleichmäßigen Streuung.

Aus den Ergebnissen der Sensitivitätsanalyse der Schädigungsfaktoren kann geschlussfolgert werden, dass der qualitative Verlauf der Gesamtschädigung maßgeblich vom SOC-Hub abhängt und dieser somit einen wichtigen Stellhebel in der Systemauslegung darstellt. Zur Vermeidung einer beschleunigten Alterung sollte der SOC-Hub über dem Tag möglichst nicht ansteigen, was einer ladungserhaltenden Betriebsstrategie der Batterie entspricht. Dafür müssen die Freiheitsgrade Batteriekapazität und Nachladung entsprechend aufeinander abgestimmt werden, was mittels den im folgenden Abschnitt durchzuführenden Optimierungsrechnungen erreicht wird.

\subsection{Mehrdimensionale Optimierung}

In Kapitel 5.1 wurden verschiedene Betriebsszenarien und Betriebsprogramme definiert, berechnet und analysiert. Dabei wurde jedoch stets nur eine Systemkonfiguration bestehend aus Batteriegröße (135 kWh), Ladepunkten (LP 1/1) und Ladeleistung (200 kW) betrachtet. In diesem Abschnitt wird nun eine Variation dieser Parameter durchgeführt mit dem Ziel der Ermittlung einer kostenoptimalen Systemkonfiguration der in dieser Arbeit beispielhaft betrachteten Linie.

\subsubsection{Optimierung auf Grundlage des Basisszenarios}

Es werden zunächst die betrieblichen und technischen Randbedingungen des Basisszenarios verwendet. Diese beinhalten im Wesentlichen die Annahmen einer konstanten Außentemperatur von $15^{\circ} \mathrm{C}$, keine Ausfallszenarien und keine Ruhetage. Für die Optimierungsrechnungen wird eine Makrozeitschrittweite von $\Delta \tau_{\text {makro }}=10 \mathrm{~d}$ gewählt. Für die in Kapitel 3.4 definierten Variationsparameter wird der Parameterraum gemäß Tabelle 5-10 definiert

\footnotetext{
${ }^{18}$ Die Streuung der Werte ist auf die nicht-linear verlaufende Alterung der Batterie aufgrund der steigenden elektro-thermischen Belastung zurückzuführen.
} 
Tabelle 5-10: Grenzen der Variationsparameter

\begin{tabular}{ccc}
\hline $\begin{array}{c}\text { Energieinhalt der } \\
\text { Batterie }\end{array}$ & Ladepunkte & Ladeleistung \\
\hline $68 \mathrm{kWh}-472 \mathrm{kWh}$ & $0 \mathrm{LP}-2 \mathrm{LP}$ & $50 \mathrm{~kW}-500 \mathrm{~kW}$ \\
\hline
\end{tabular}

Es werden alle möglichen Kombinationen der Variationsparameter innerhalb der gesetzten Grenzen berechnet und anschließend mittels des Kostenmodells aus Kapitel 4.3 bewertet. Grundlage dafür ist der globale Programmablaufplan aus Abbildung 3-3. Das Kostenmodell wird dafür mit den Werten in Abbildung 5-26 parametriert. Die Kostensätze orientieren sich an den in (Knote et al., 2017) und in (Bloomberg, 2018) dargelegten Werten. Grundsätzlich sei jedoch angemerkt, dass es sich hierbei nur um eine beispielhafte Parametrierung zur Demonstration der Funktionalität der nachfolgenden Optimierungsrechnungen handelt.

Für die Fahrzeuge (ohne Batterien) werden Investkosten von 200 TEUR je Fahrzeug angesetzt. Die Kosten einer Traktionsbatterie sind mit 500 EUR/kWh sowohl im Invest als auch im Re-Invest parametriert. Eine jährliche Kostenreduzierung könnte jedoch ebenso hinterlegt werden. Für die Festanteile der Ladepunkte sollen unterschiedliche Kostensätze gelten. LP 1 wird mit 85 TEUR und LP 2 mit 50 TEUR parametriert. Die leistungsabhängigen Investkosten werden mit $600 \mathrm{EUR} / \mathrm{kW} / \mathrm{LP}$ für beide Ladepunkte gleich angenommen. Die Energiekosten sind mit 0,20 EUR/kWh parametriert.

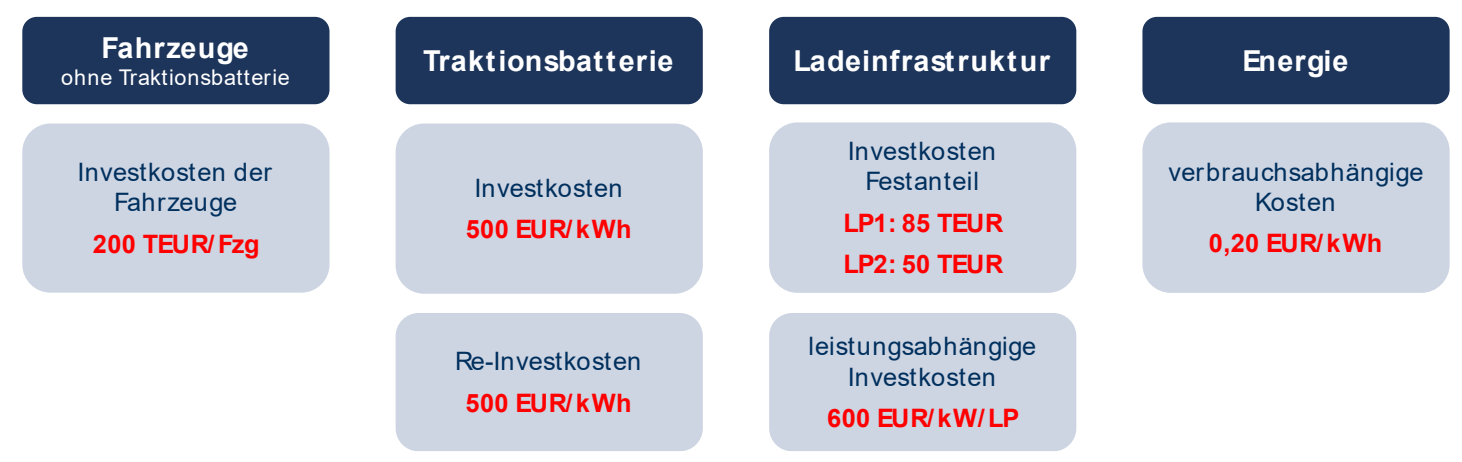

Abbildung 5-26: Parametriertes Kostenmodell

Die Ergebnisse der Optimierungsrechnung sind in Abbildung 5-27 dargestellt. Daraus geht hervor, dass eine Ausführung der betrachteten Linie als Depot-Charger innerhalb der definierten Grenzen der Variationsparameter nicht realisierbar ist. Wird nur der Ladepunkt 2 mit 10 min Ladezeit gewählt (LP 0/1), so gibt es nur wenige realisierbare Konfigurationen. Diese weisen jeweils einen vergleichsweise großen Energieinhalt der Batterie sowie eine hohe Ladeleistung auf. Bei Verwendung des Ladepunktes 1 (LP 1/0) 
stehen aufgrund der längeren Ladezeit von 15 min deutlich mehr realisierbare Konfigurationen zur Verfügung. Bei Verwendung beider Ladepunkte (LP 1/1) können demnach die meisten Konfigurationen realisiert werden. Für alle technisch realisierbaren Systemkonfigurationen werden die resultierenden Systemkosten ermittelt. Aus Abbildung 5-27 geht hervor, dass die Kostenverläufe keine markanten Sprünge oder Unstetigkeiten aufweisen. Das Kostenminimum ist nicht unmittelbar ablesbar, kann jedoch mittels einer Minimum-Suchfunktion gefunden werden.
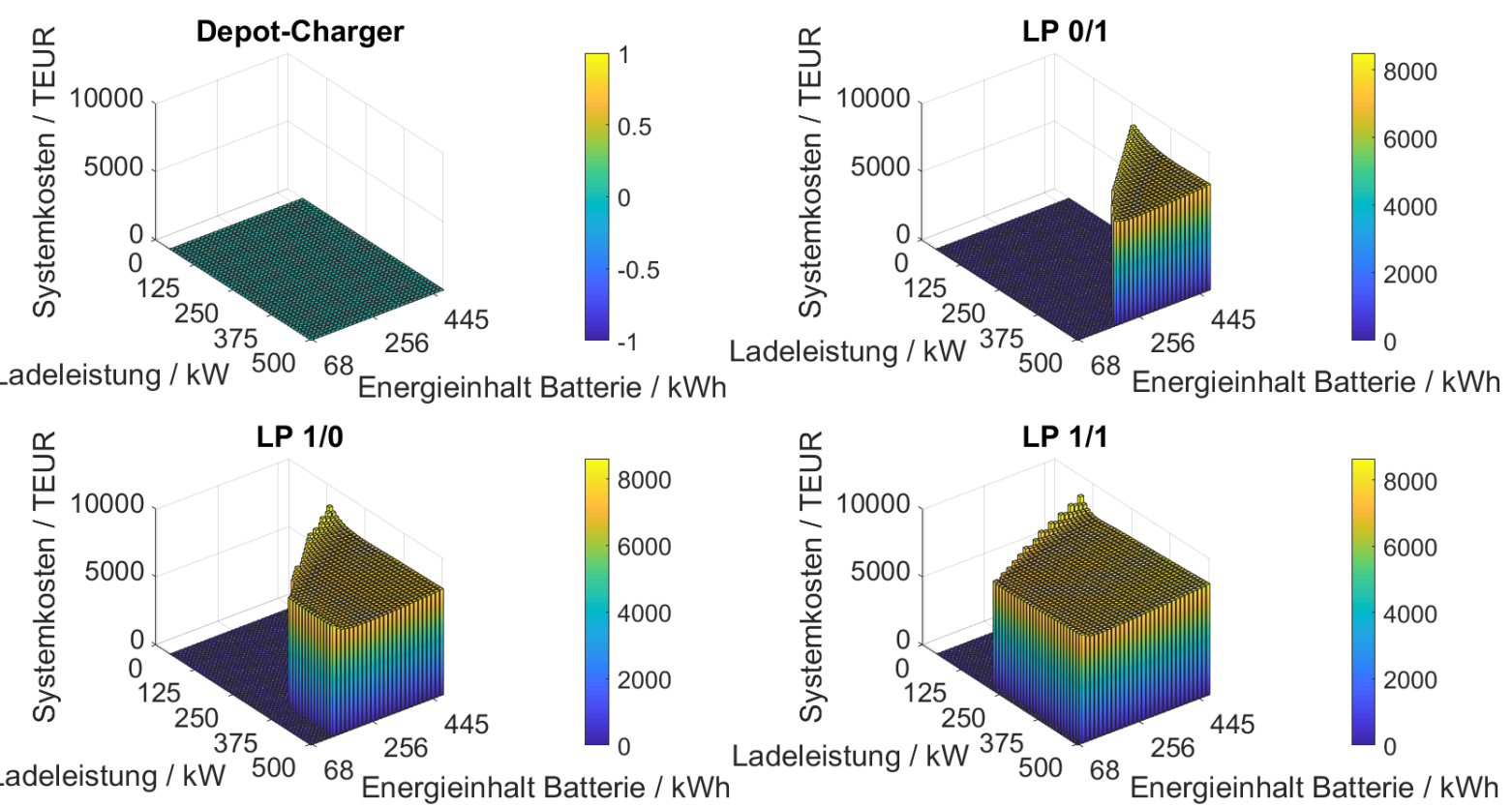

\section{Abbildung 5-27: Optimierungsergebnisse (Basisszenario)}

Zur detaillierten Analyse werden die Optimierungsergebnisse in eine zweidimensionale Ansicht überführt. Für jede Ladeleistung sind das Kostenminimum und der jeweils zugehörige Energieinhalt der Batterie in Abbildung 5-28 dargestellt. Aus der Abbildung geht hervor, dass das absolute Kostenminimum bei Ladepunktkombination LP 1/1 bei einer Ladeleistung von $200 \mathrm{~kW}$ liegt. Der dazugehörige Energieinhalt der Batterie beträgt $162 \mathrm{kWh}$. Die resultierenden Systemkosten betragen 7,314 Mio. EUR. Es ergibt sich eine Lebensdauer der Batterie von 1931 Betriebstagen, was 5,3 Jahren entspricht. Die Ergebnisse der optimalen Systemkonfiguration sind in Tabelle 5-11 zusammengefasst. 
Tabelle 5-11: Zusammenfassung der Ergebnisse der optimalen Konfiguration (Basisszenario)

\begin{tabular}{ll}
\hline Ladepunktkombination & LP 1/1 \\
Energieinhalt Batterie & $162 \mathrm{kWh}$ \\
Ladeleistung & $200 \mathrm{~kW}$ \\
zu installierende Leistung & $223 \mathrm{~kW}$ \\
am Netzanschlusspunkt & \\
Systemkosten & 7,314 Mio. EUR \\
Lebensdauer Batterie & 5,3 Jahre \\
\hline
\end{tabular}
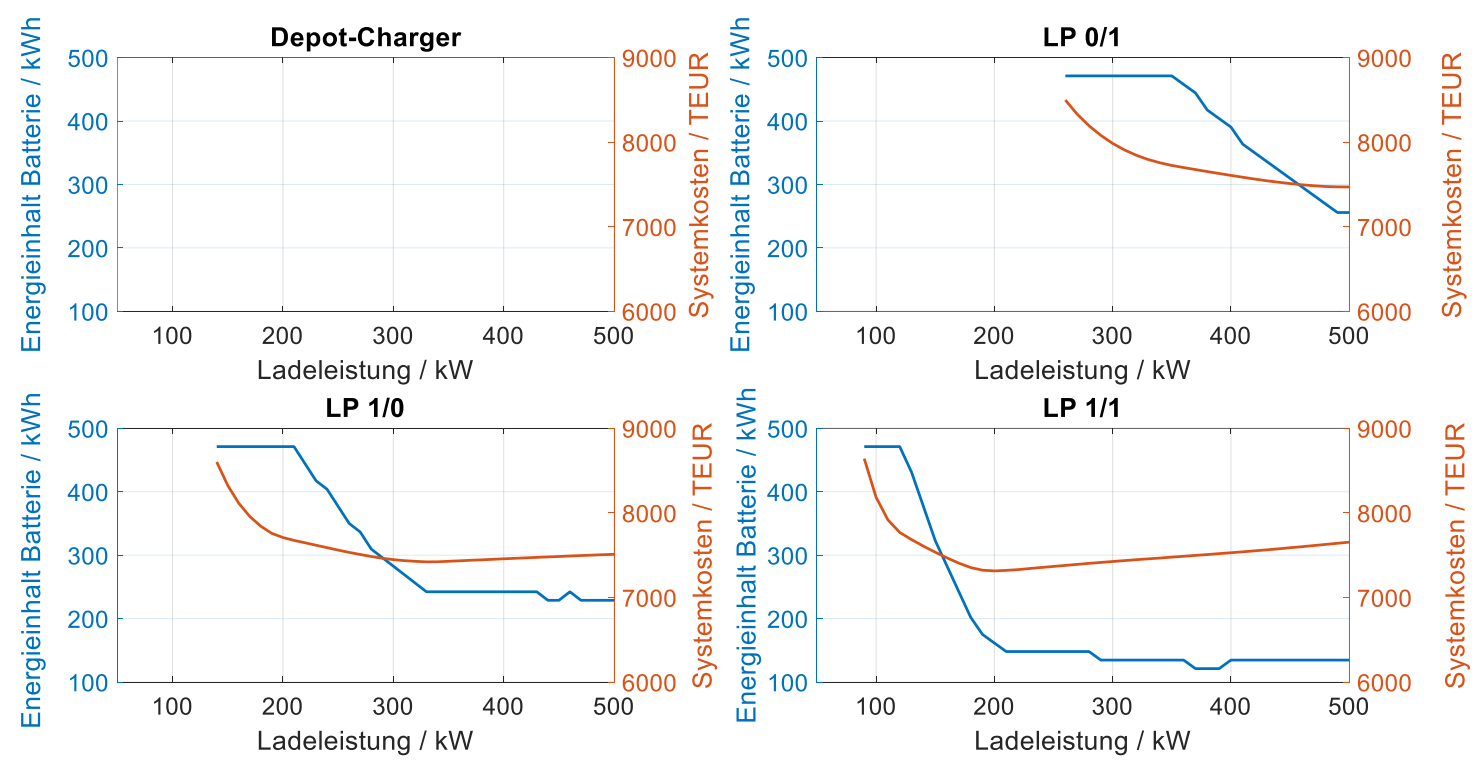

\section{Abbildung 5-28: Kostenminimum für jede Ladeleistung mit zugehörigem Energieinhalt der Batterie (Basisszenario)}

Der resultierende SOC-Verlauf der optimalen Systemkonfiguration ist in Abbildung 5-29 dargestellt. Es ist erkennbar, dass die optimale Konfiguration einen ladungserhaltenden SOC-Verlauf während eines Betriebstages aufweist. Die Ladeleistung ist gerade so groß, dass die Batterie (auch im gealterten Zustand) immer wieder auf (nahezu) $S O C_{\max }=0,9$ aufgeladen wird. Es handelt sich somit um eine Speicherbetriebsstrategie, welche nur eine alterungsbedingte Vergrößerung des SOC-Hubs gemäß Abbildung 5-23 aufweist. Es wird somit der in Kapitel 5.2.2 ermittelte Zusammenhang bestätigt, nach dem der SOC-Hub die wesentlich Steuerungsgröße für die Batteriealterung im Betrieb eines Elektrobusses ist. In Abbildung 5-30 ist der SOC- 
Verlauf mit einer Ladeleistung von $190 \mathrm{~kW}$ gegeben ${ }^{19}$. Daraus geht hervor, dass die als optimal ermittelte Ladeleistung von 200 kW genau die Grenze bildet, an welcher ein ladungserhaltender SOC-Verlauf ermöglicht werden kann.

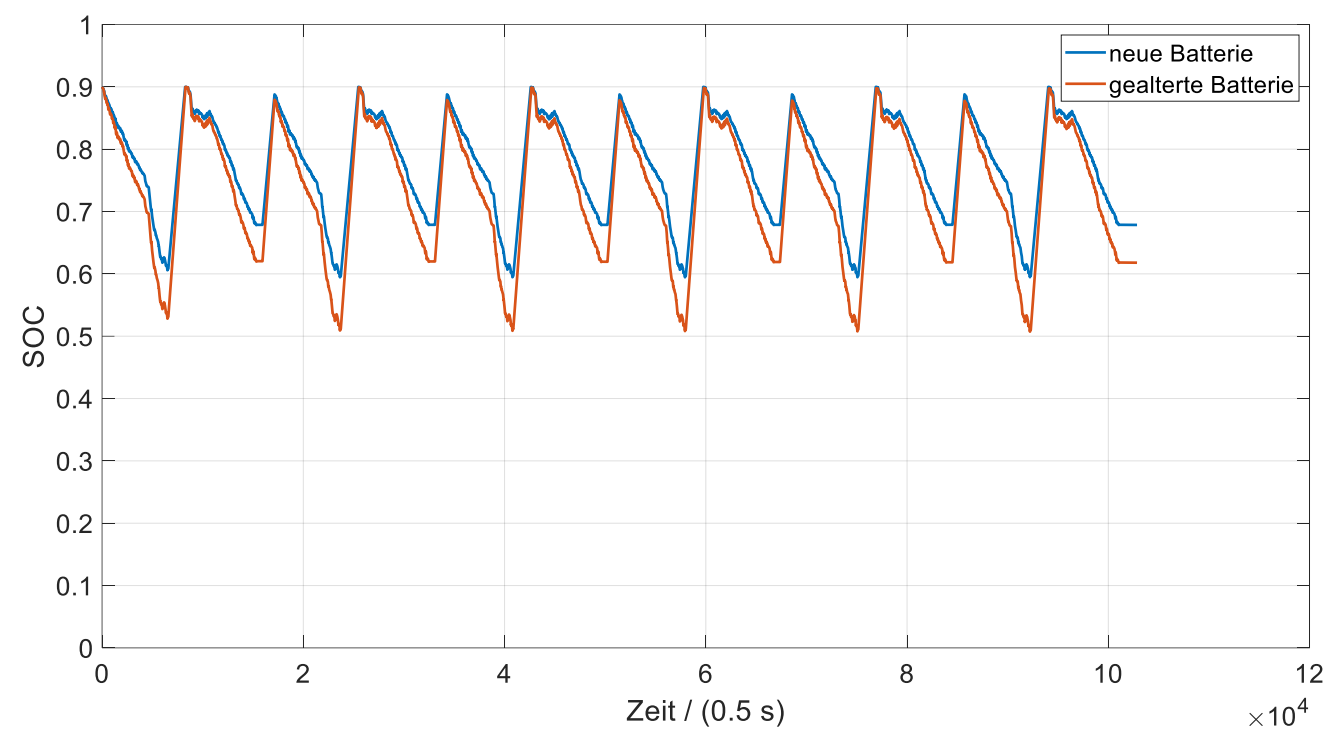

Abbildung 5-29: Resultierender SOC-Verlauf der optimalen Konfiguration (Basisszenario)

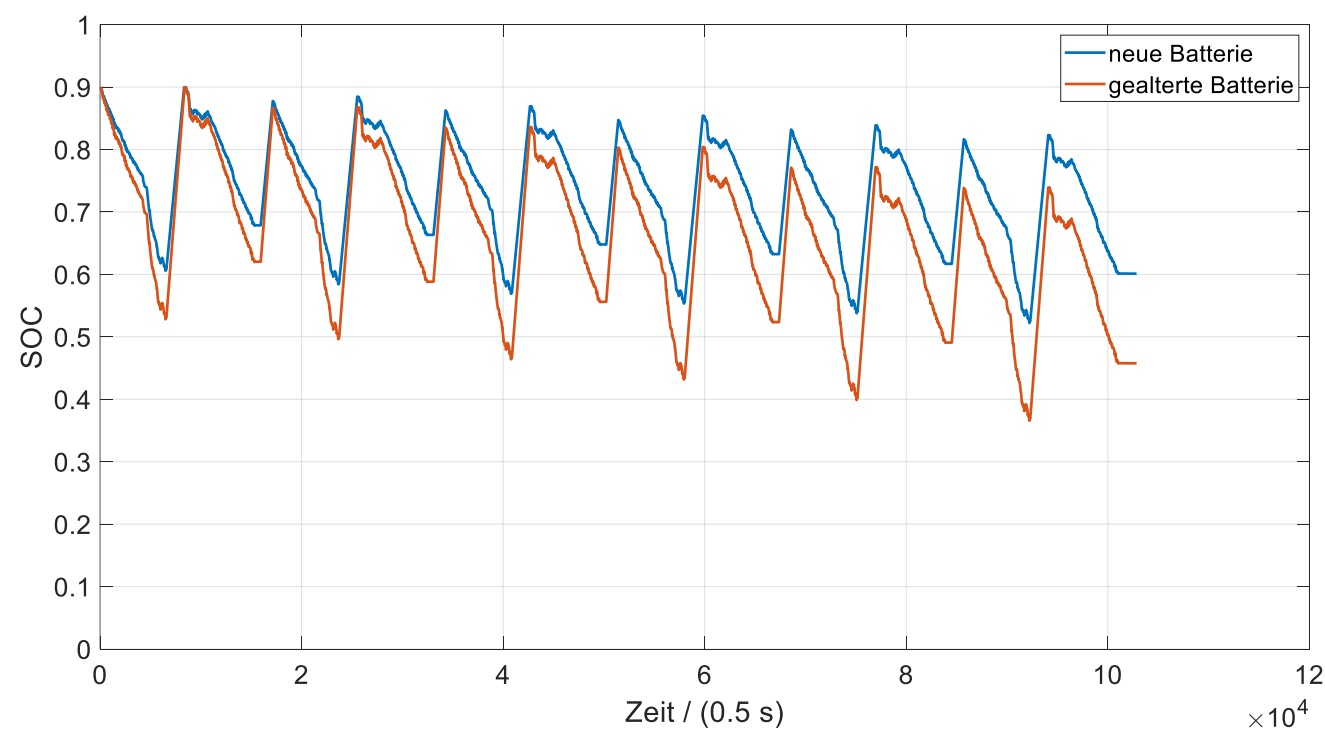

Abbildung 5-30: Resultierender SOC-Verlauf der Konfiguration 162 kWh, LP 1/1, 190 kW (Basisszenario)

${ }^{19}$ Der Energieinhalt der Batterie beträgt 162 kWh und es werden beide Ladepunkte verwendet. Dies entspricht den Werten der optimalen Systemkonfiguration im Basisszenario. 
Es werden abschließend die optimalen Systemkonfigurationen der Ergebnisteilmengen LP 1/0 und LP 0/1 analysiert. Diese sind in Tabelle 5-12 zusammengefasst.

Tabelle 5-12: Optimale Systemkonfigurationen der Teilmengen LP $1 / 0$ und LP 0/1

\begin{tabular}{lll}
\hline & \multicolumn{1}{c}{ LP 1/0 } & \multicolumn{1}{c}{ LP 0/1 } \\
\hline Energieinhalt Batterie & $242 \mathrm{kWh}$ & $256 \mathrm{kWh}$ \\
Ladeleistung & $330 \mathrm{~kW}$ & $500 \mathrm{~kW}$ \\
$\begin{array}{l}\text { zu installierende Leistung } \\
\text { am Netzanschlusspunkt }\end{array}$ & $367 \mathrm{kWh}$ & $556 \mathrm{~kW}$ \\
Systemkosten & 7,421 Mio. EUR & 7,470 Mio. EUR \\
Lebensdauer Batterie & 7,09 Jahre & 7,78 Jahre \\
\hline
\end{tabular}

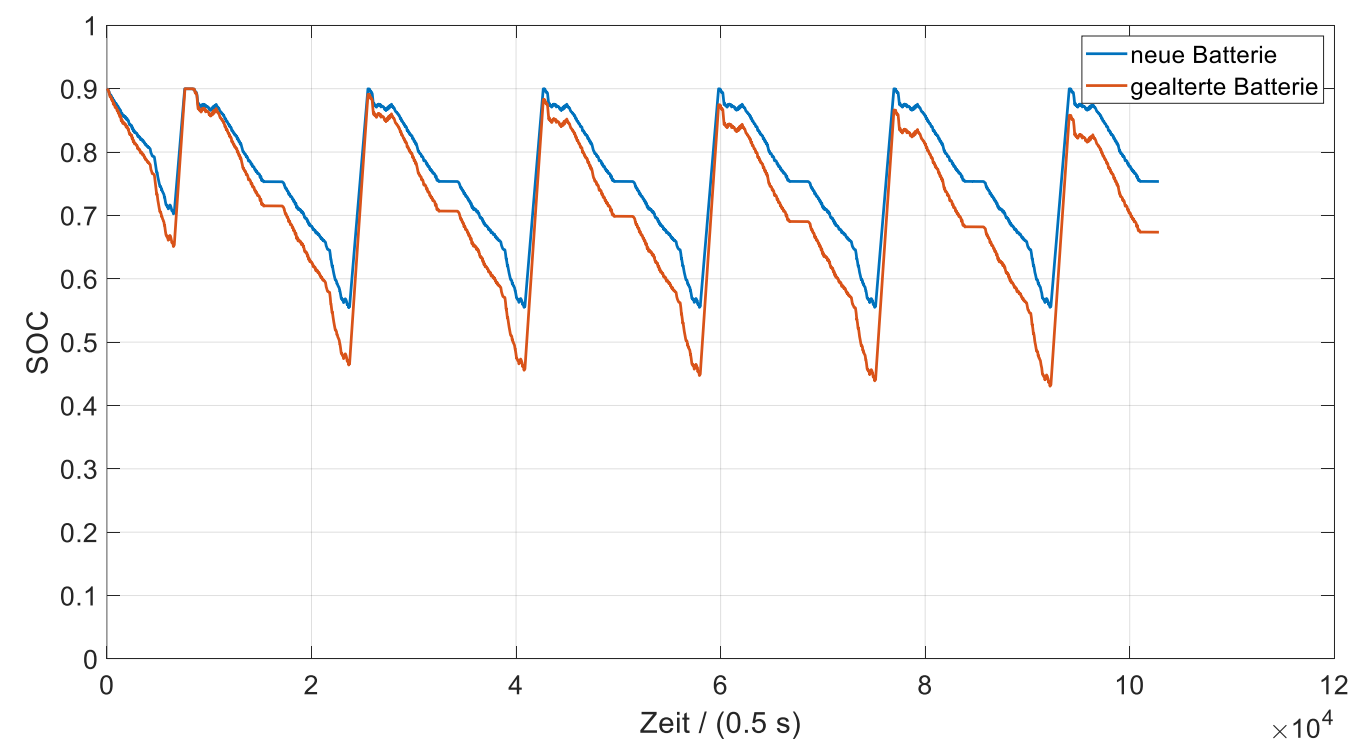

\section{Abbildung 5-31: Resultierender SOC-Verlauf der optimalen Konfiguration bei LP 1/0 (Basisszenario)}

Aus Abbildung 5-28 geht hervor, dass die Systemkosten von LP 1/0 einen ähnlichen Verlauf wie LP $1 / 1$ aufweisen. Die optimale Systemkonfiguration liegt bei einer Ladeleistung von $330 \mathrm{~kW}$ und der zugehörige Energieinhalt beträgt $242 \mathrm{kWh}$. Der SOCVerlauf dieser Systemkonfiguration ist in Abbildung 5-31 dargestellt. Daraus geht hervor, dass mit dieser Systemkonfiguration nur ein ladungserhaltender SOC-Verlauf für eine neue, nicht jedoch für eine gealterte Batterie ermöglicht wird. Damit muss die 
oben getroffene Aussage teilweise korrigiert werden. Die grundlegende Tendenz bleibt jedoch bestehen, wonach die optimale stets in der Nähe eines ladungserhaltenden SOC-Verlaufs während eines Betriebstages liegt. Aus Abbildung 5-28 geht hervor, dass der Verlauf der Kostenkurve kein lokales Minimum aufweist. Das Optimum liegt am Ende der Kurve bei 500 kW Ladeleistung, was der Grenze des Parameterraums entspricht. Der zugehörige Batterieenergieinhalt beträgt $256 \mathrm{kWh}$. Der resultierende SOCVerlauf ist in Abbildung 5-32 dargestellt. Dieser weist ebenfalls nur bei einer neuen Batterie einen ladungserhaltenden SOC-Verlauf auf.

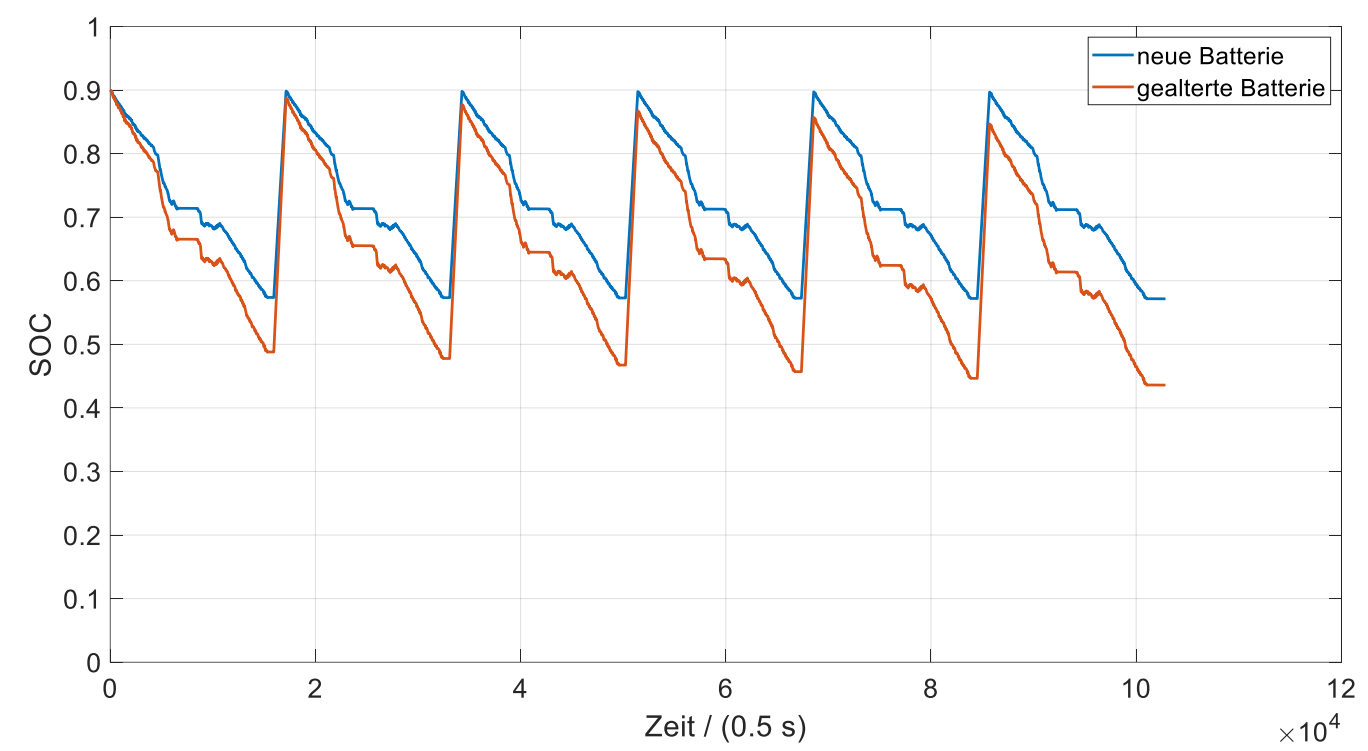

\section{Abbildung 5-32: Resultierender SOC-Verlauf der optimalen Konfiguration bei LP 0/1 (Basisszenario)}

Für eine detaillierte Analyse des resultierenden Kostenverlaufs sind dessen Bestandteile in Abbildung 5-33 für LP 1/1 über der Ladeleistung einzeln aufgetragen. Der Verlauf der Gesamtsystemkosten fällt im Bereich von 90 kW bis 200 kW stark ab. Dies ist vor allem dem Umstand geschuldet, dass in diesem Bereich der Energieinhalt der Batterie mit steigender Ladeleistung abnimmt und somit auch die Batteriekosten fallen (Vgl. Abbildung 5-28). Ab $200 \mathrm{~kW}$ kann der Batterieenergieinhalt nicht mehr signifikant verkleinert werden, da sonst die Mindestenergiemenge zur Fahrt zwischen den Ladepunkten nicht ausreicht. Im Bereich von $200 \mathrm{~kW}$ bis $500 \mathrm{~kW}$ findet kaum noch eine Änderung der Batteriegröße statt, sodass dadurch auch kein erkennbarer Einfluss auf den Verlauf der Gesamtsystemkosten entsteht. Vielmehr geht aus der Abbildung hervor, dass die Kosten für die Ladeinfrastruktur zwar den kleinsten Anteil darstellen, jedoch linear zur Ladeleistung ansteigen. Im vorderen Bereich von $90 \mathrm{~kW}$ bis $200 \mathrm{~kW}$ wird der Anstieg der Ladeinfrastrukturkosten durch den starken Abfall der Batteriekosten überlagert. Im hinteren Bereich von $200 \mathrm{~kW}$ bis $500 \mathrm{~kW}$ dominiert der Anstieg 
der Infrastrukturkosten den Kurvenverlauf der Gesamtsystemkosten. Folgerichtig ergibt sich das Minimum der Kurve an der Stelle, an der der starke Abfall der Batteriekosten endet. Dieses liegt im hier betrachteten Beispiel bei einer Ladeleistung von $200 \mathrm{~kW}$. Die Kosten für die Fahrzeuge (ohne Batterie) verlaufen konstant auf einem Wert, da diese nur abhängig von der Umlaufzeit und der Taktzeit sind und demnach nicht von den Variationsparametern beeinflusst werden. Den größten Anteil an den Gesamtsystemkosten stellen die Energiekosten dar. Im vorderen Bereich der Kurve bis $200 \mathrm{~kW}$ Ladeleistung verlaufen diese leicht fallend. Dies ist vor allem auf die leichter werdenden Fahrzeuge aufgrund des reduzierten Batteriegewichts zurückzuführen. Gleichzeitig wird der Verlauf jedoch von der steigenden Ladeleistung überlagert, welche aufgrund der höheren Verlustleistung einen leichten Anstieg der Energiekosten bedingt. Dementsprechend steigen diese im Bereich von $200 \mathrm{~kW}$ bis $500 \mathrm{~kW}$ sukzessive an. Zusammengefasst haben die Energiekosten jedoch einen geringen Einfluss auf den qualitativen Verlauf der Gesamtkosten.

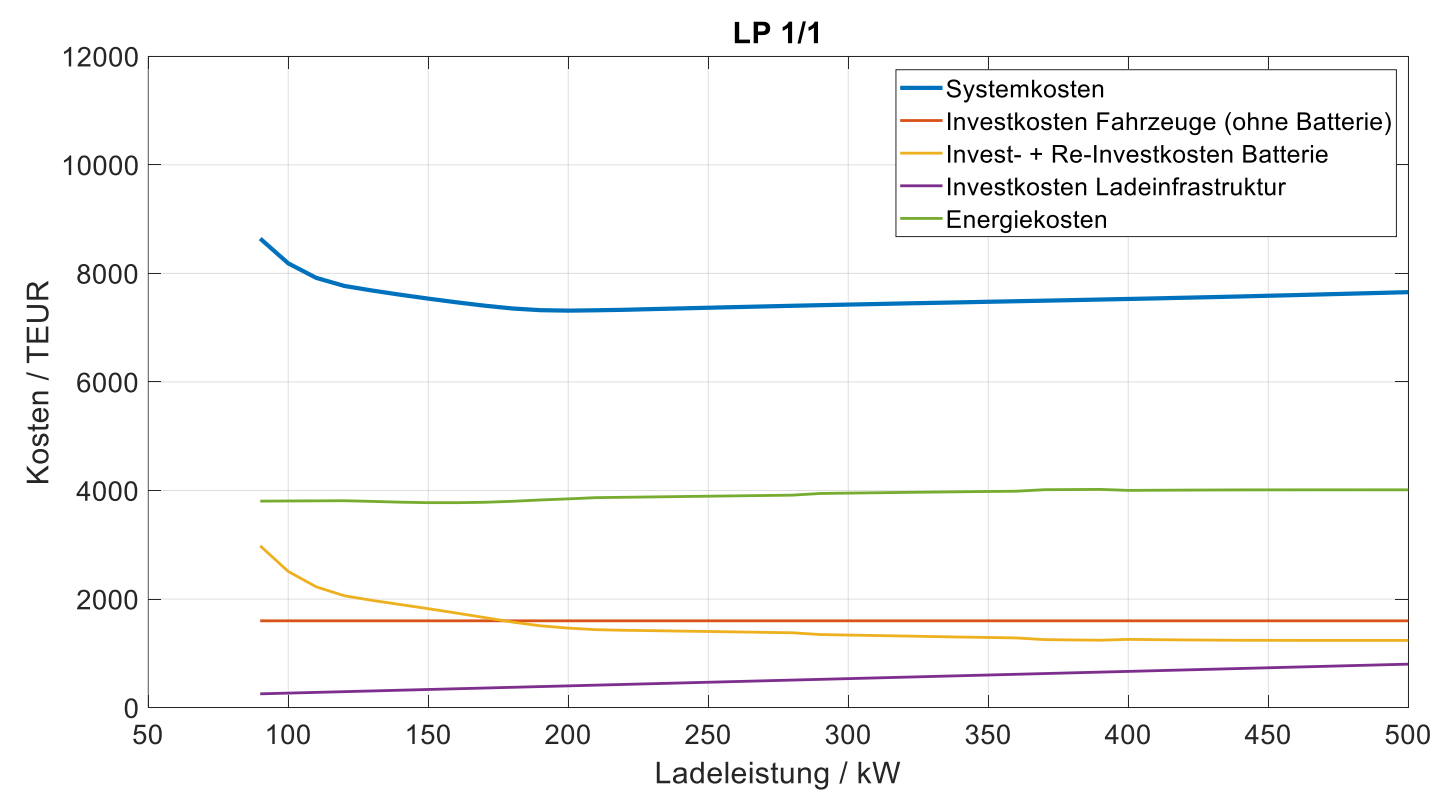

Abbildung 5-33: Kostenbestandteile (LP 1/1, Basisszenario)

\subsubsection{Optimierung mit verteilten Temperaturen, Ruhetagen und Ausfallszenarien}

In diesem Abschnitt wird die Optimierungsrechnung für das erweiterte Szenario nach Abbildung 5-1 durchgeführt. Gegenüber den Berechnungen auf Grundlage des Basisszenarios in Kapitel 5.3.1 werden folgende Randbedingungen gemäß Tabelle 5-13 hinzugefügt. 
Tabelle 5-13: Zusätzliche Randbedingungen zur Optimierungsrechnung des erweiterten Szenarios

Ruhetage

Ausfallszenario

Temperaturverteilung

Batterieheizung ein Ruhetag pro Woche

(entspricht 313 Betriebstagen pro Jahr)

2 Betriebstage mit Ausfallszenario pro Woche

Monatsmittelwerte (Vgl. Abbildung 5-14, links)

Sollwert: $25^{\circ} \mathrm{C}$

Hysterese: $5^{\circ} \mathrm{C}$

Die Ergebnisse der Optimierungsrechnung sind in Abbildung 5-34 dargestellt. Daraus geht hervor, dass keine technisch realisierbaren Lösungen für eine Ausführung als Depot-Charger existieren. Für die übrigen Ladepunktkombinationen stehen deutlich weniger realisierbare Konfigurationen als im Basisszenario zur Verfügung. Dies ist vor allem auf die Ausfallszenarien zurückzuführen, da dadurch ein deutlich größerer SOCBereich erforderlich ist.
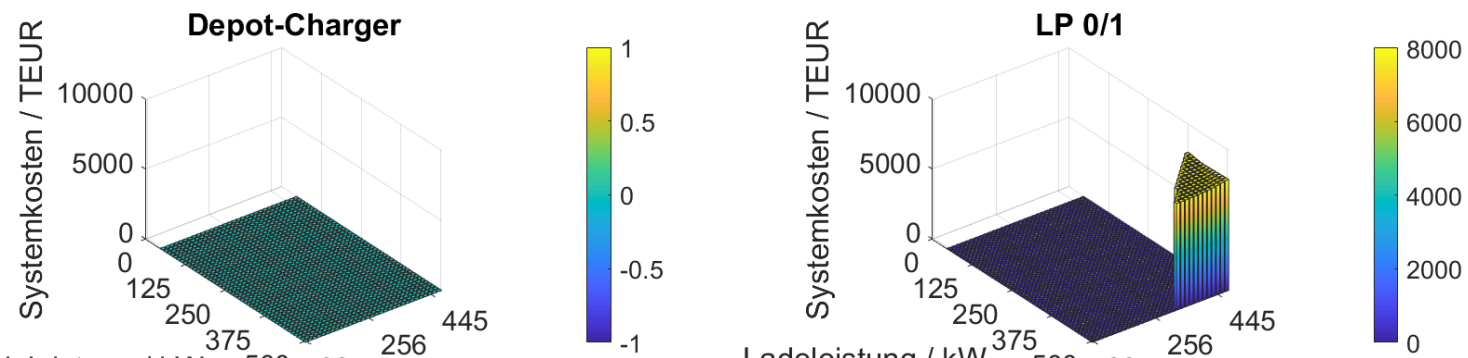

Ladeleistung / kW 50068 Energieinhalt Batterie / kWh Ladeleistung / kW 50068 Energieinhalt Batterie / kWh
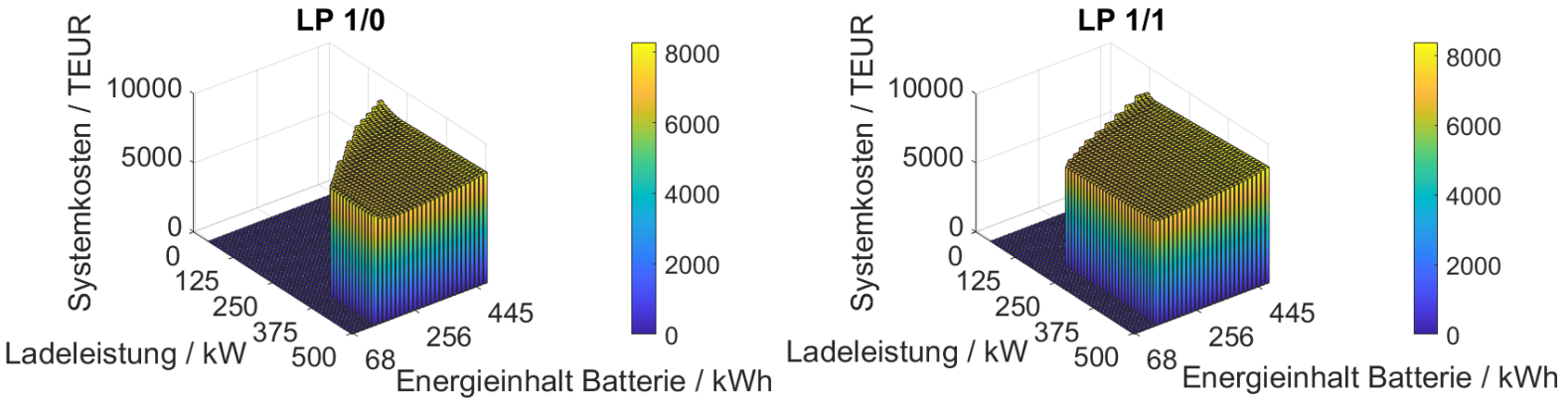

Abbildung 5-34: Optimierungsergebnisse (erweitertes Szenario)

Die kostenoptimale Konfiguration kann analog zum Basisszenario mittels einer Minimumsuchfunktion ermittelt werden. In Abbildung 5-35 sind für jede Ladeleistung die 
minimalen Systemkosten und der jeweils zugehörige Energieinhalt der Batterie dargestellt. Aus der Abbildung geht hervor, dass das absolute Kostenminimum bei Ladepunktkombination LP 1/1 bei einer Ladeleistung von $200 \mathrm{~kW}$ liegt. Der dazugehörige Energieinhalt der Batterie beträgt $162 \mathrm{kWh}$. Die resultierenden Systemkosten betragen 7,233 Mio. EUR. Es ergibt sich eine Lebensdauer der Batterie von 2094 Betriebstagen, was 5,75 Jahren entspricht. Die Ergebnisse der optimalen Systemkonfiguration des erweiterten Szenarios sind in Tabelle 5-14 zusammengefasst. Es ist erkennbar, dass das Kostenminimum des erweiterten Szenarios bei exakt der gleichen Systemkonfiguration wie beim Basisszenario liegt. Ein Vergleich der beiden Optimierungsrechnungen erfolgt in Kapitel 5.3.3.
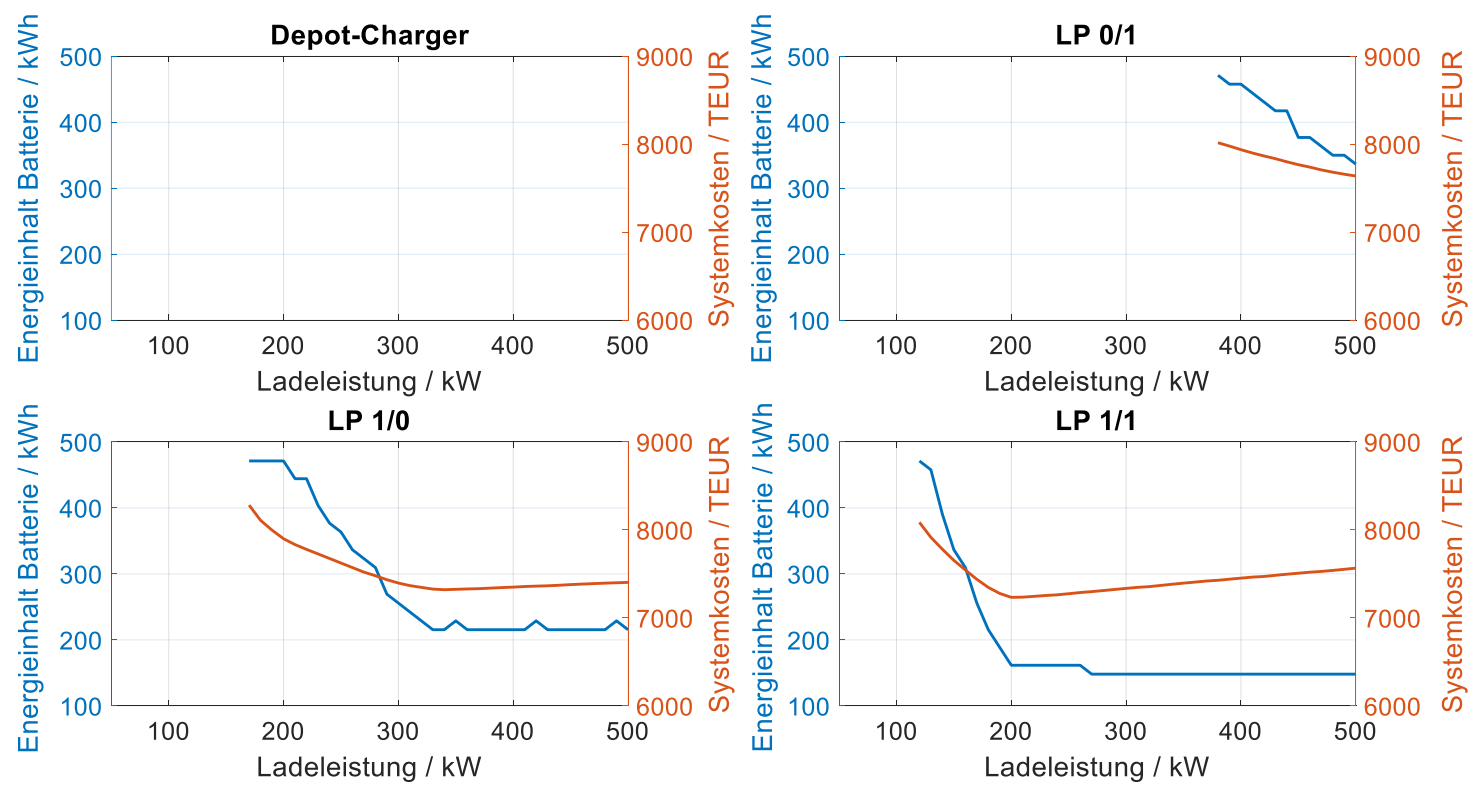

\section{Abbildung 5-35: Kostenminimum für jede Ladeleistung mit zugehörigem Energieinhalt der Batterie (erweitertes Szenario)}

Tabelle 5-14: Zusammenfassung der Ergebnisse der optimalen Konfiguration (erweitertes Szenario)

\begin{tabular}{ll}
\hline Ladepunktkombination & LP 1/1 \\
Energieinhalt Batterie & $162 \mathrm{kWh}$ \\
Ladeleistung & $200 \mathrm{~kW}$ \\
zu installierende Leistung & \\
am Netzanschlusspunkt & $223 \mathrm{~kW}$ \\
Systemkosten & 7,233 Mio. EUR \\
Lebensdauer Batterie & 5,75 Jahre \\
\hline
\end{tabular}


In Abbildung 5-36 ist der resultierende SOC-Verlauf der kostenoptimalen Systemkonfiguration mit Ausfallszenario gegeben. Deutlich erkennbar ist der vergrößerte SOC-Hub aufgrund des Ausfallszenarios im Vergleich zum Basisszenario. Daraus geht hervor, dass der SOC-Verlauf sowohl im ersten Teil des simulierten Tages (vor dem Ausfallszenario) als auch im zweiten Tagesteil einen jeweils annähernd ladungserhaltenden SOC-Verlauf aufweist.

In Abbildung 5-37 sind analog zum Basisszenario die einzelnen Kostenbestandteile für die jeweiligen Ladeleistungen (und jeweils dazugehörigem Batterieenergieinhalt) dargestellt. Der qualitative Verlauf entspricht dem des Basisszenarios. Während der Abschnitt bis zur optimalen Ladeleistung von den fallenden Batteriekosten geprägt ist, dominieren anschließend die Kosten der Ladeinfrastruktur.

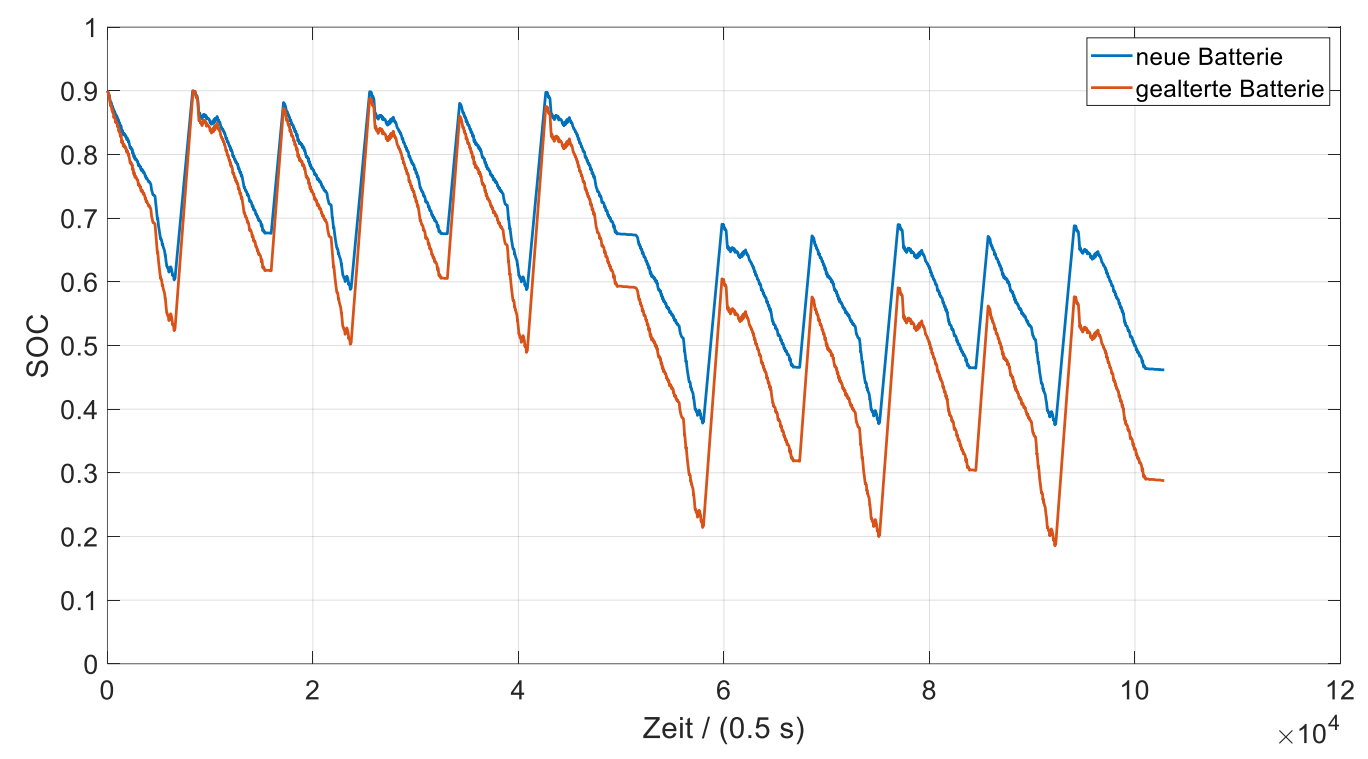

\section{Abbildung 5-36: Resultierender SOC-Verlauf der optimalen Konfiguration mit Ausfallszenario (erweitertes Szenario)}

\subsubsection{Vergleich und Bewertung der Optimierungsrechnungen}

In den Kapiteln 5.3.1 und 5.3.2 wurden Optimierungsrechnungen für zwei unterschiedliche Szenarien durchgeführt, das Basisszenario und ein erweitertes Szenario. Aus den Ergebnissen geht hervor, dass die kostenoptimale Systemkonfiguration bei beiden Rechnungen gleich ist. Es wurden jeweils zwei Ladepunkte entlang der Strecke mit einer Ladeleistung von je $200 \mathrm{~kW}$ und ein Batterieenergieinhalt von $162 \mathrm{kWh}$ ermittelt. Es kann demnach geschlussfolgert werden, dass die Erweiterung des Basisszenarios um betriebliche und technische Szenarien im hier gezeigten Beispiel keinen 
Einfluss auf die optimale Systemkonfiguration hat. Es entstehen jedoch Auswirkungen auf die resultierende Lebensdauer der Batterie. Diese liegt im erweiterten Szenario mit 5,75 Jahren um 8,5\% höher als im Basisszenario. Eine Überlagerung verschiedener Umstände führt zu diesem Ergebnis. Im erweiterten Szenario wurde ein Ruhetag pro Woche berücksichtigt, welcher positiv auf die Lebensdauer wirkt (Vgl. Kapitel 5.1.3). Dagegen wirken sich die Tage mit Ausfallszenario aufgrund des größeren SOC-Hubs negativ auf die Lebensdauer aus ( $\mathrm{Vgl}$. Kapitel 5.1.4). Des Weiteren wurde sowohl mit verteilten Temperaturen als auch mit Batterieheizung gerechnet. Aus den Ausführungen in Kapitel 5.1.5 geht hervor, dass Batterietemperaturen oberhalb von $15^{\circ} \mathrm{C}$ zu einer deutlichen Beschleunigung der Batteriealterung führen. Aus der längeren Lebensdauer im erweiterten Szenario gegenüber dem Basisszenario kann geschlussfolgert werden, dass die negativen Auswirkungen auf die Batteriealterung (Ausfallszenario und Batterieheizung mit $25^{\circ} \mathrm{C}$ ) von der positiven Wirkung des Ruhetages überlagert werden. Für praktische Anwendungen kann demnach festgehalten werden, dass für die Gesamtbilanz der Batteriealterung die wöchentliche Einsatzplanung eine wichtige Rolle einnimmt, wohingegen der operative Betrieb in Form von Verspätungen eine vergleichsweise untergeordnete Rolle spielt. Die erhöhte Lebensdauer führt zu einer Reduzierung der Systemkosten um ca. 1,1\%. Die Ergebnisse der beiden Optimierungsrechnungen sind in Tabelle 5-15 vergleichend gegenübergestellt.

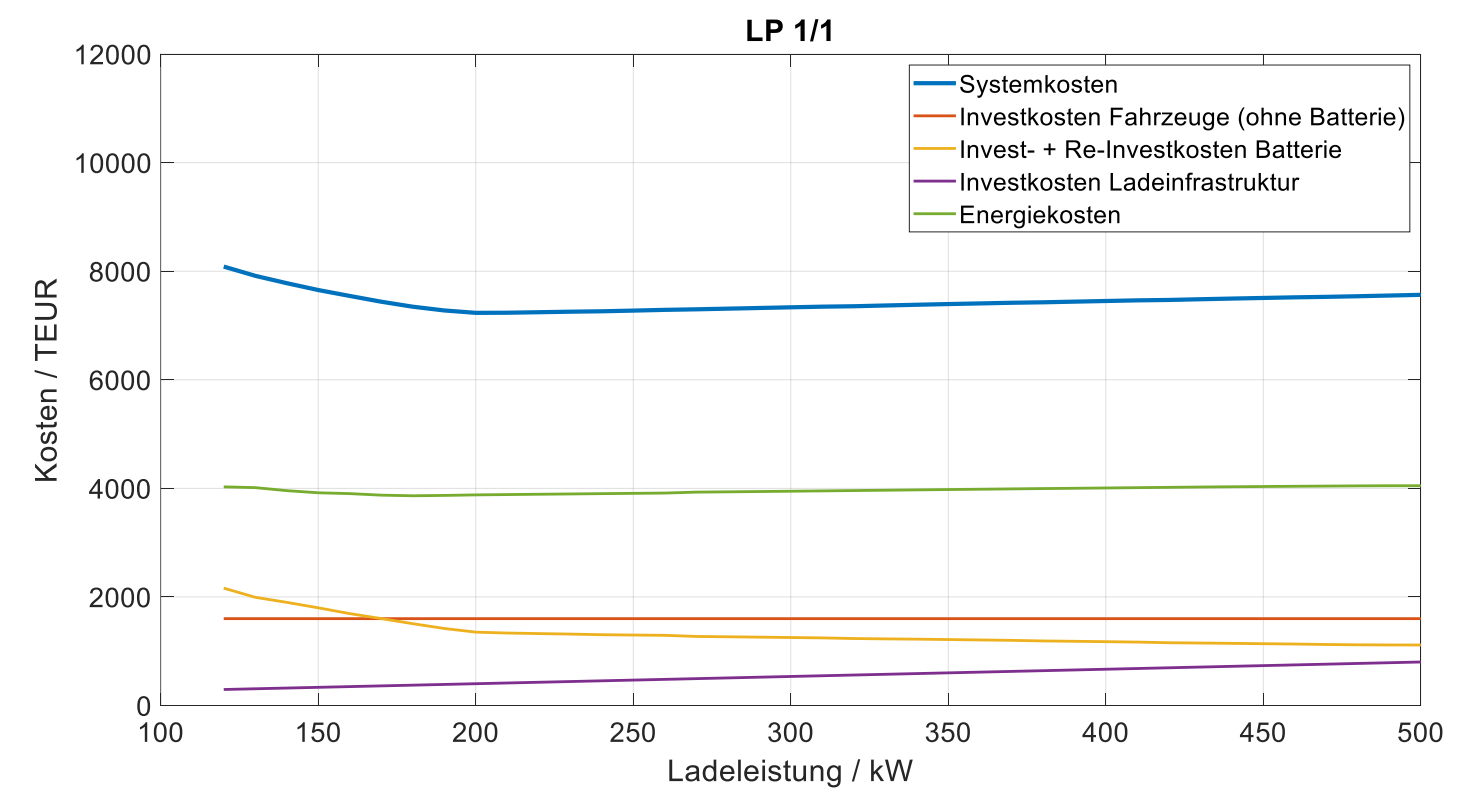

Abbildung 5-37: Kostenbestandteile (LP 1/1, erweitertes Szenario)

Für das erweiterte Szenario wurde die Optimierungsrechnung zusätzlich für die Sollwerte $15^{\circ} \mathrm{C}$ und $20^{\circ} \mathrm{C}$ der Batterieheizung durchgeführt. Die Ergebnisse in Form der dreidimensionalen Balkendiagramme sind in Anhang A.1 und A.2 dargestellt. Die 
jeweils optimalen Systemkonfigurationen sind in Tabelle 5-16 zusammengefasst und gegenübergestellt.

Tabelle 5-15: Vergleich der Optimierungsrechnungen

\begin{tabular}{|c|c|c|}
\hline & Basisszenario & $\begin{array}{l}\text { erweitertes } \\
\text { Szenario }\end{array}$ \\
\hline Ladepunktkombination & LP $1 / 1$ & LP $1 / 1$ \\
\hline Energieinhalt Batterie & $162 \mathrm{kWh}$ & 162 kWh \\
\hline Ladeleistung & $200 \mathrm{~kW}$ & $200 \mathrm{~kW}$ \\
\hline $\begin{array}{l}\text { zu installierende Leistung } \\
\text { am Netzanschlusspunkt }\end{array}$ & $223 \mathrm{~kW}$ & $223 \mathrm{~kW}$ \\
\hline \multirow{2}{*}{ Systemkosten } & 7,314 Mio. EUR & 7,233 Mio. EUR \\
\hline & $100 \%$ & $98,9 \%$ \\
\hline \multirow{2}{*}{ Lebensdauer Batterie } & 5,3 Jahre & 5,75 Jahre \\
\hline & $100 \%$ & $108,5 \%$ \\
\hline
\end{tabular}

Beim Vergleich der jeweils kostenoptimalen Systemkonfigurationen bei unterschiedlichen Sollwerten der Batterieheizung fällt auf, dass sich die Ergebniswerte der Ladeinfrastruktur nicht ändern. Das Optimum beträgt in allen drei Varianten zwei Ladepunkte mit jeweils $200 \mathrm{~kW}$ Ladeleistung. Mit fallender Solltemperatur steigt der Energieinhalt der Batterie an. Dies ist auf die Temperaturabhängigkeit der Kapazität zurückzuführen. Interessant ist der Vergleich der resultierenden Systemkosten. Trotz der größten Batterie aller drei Varianten liegen die Kosten bei $15^{\circ} \mathrm{C}$ Solltemperatur am niedrigsten. Die Kosten für den höheren Energieinhalt werden durch die niedrigeren Kosten aufgrund einer längeren Lebensdauer kompensiert. Diese ergibt sich vor allem aufgrund der niedrigen Soll-Temperatur von $15^{\circ} \mathrm{C}$. Die Kostenunterschiede fallen mit max. 0,18\% jedoch sehr gering aus und stellen für praktische Anwendungen in vielen Fällen voraussichtlich kein Entscheidungskriterium dar. Daraus geht hervor, dass die Wahl einer Solltemperatur für die Batterieheizung im hier gezeigten Beispiel lediglich einen Einfluss auf die Größe der Batterie, nicht jedoch auf die Ladeinfrastruktur und auch nicht auf die Höhe der Systemkosten hat.

Aus den Ergebnissen der Optimierungsrechnungen kann geschlussfolgert werden, dass als optimale Systemkonfiguration bei beiden Szenarien eine verhältnismäßig kleine Batterie und eine moderate Ladeleistung, jeweils im Vergleich zur Größe des Parameterraums, gewählt wurden. Weder sehr große Batterien noch sehr hohe Ladeleistungen führen im betrachteten Beispiel zu einem wirtschaftlichen Optimum. 
Tabelle 5-16: Vergleich der Optimierungsrechnungen mit weiteren Batterieheizungssolltemperaturen

\begin{tabular}{|c|c|c|c|c|}
\hline \multirow[b]{2}{*}{ Sollwert Batterieheizung } & \multirow{2}{*}{$\begin{array}{c}\begin{array}{c}\text { Basis- } \\
\text { szenario }\end{array} \\
--\end{array}$} & \multicolumn{3}{|c|}{ erweitertes Szenario } \\
\hline & & $25^{\circ} \mathrm{C}$ & $20^{\circ} \mathrm{C}$ & $15^{\circ} \mathrm{C}$ \\
\hline Ladepunktkombination & LP $1 / 1$ & LP $1 / 1$ & LP $1 / 1$ & LP $1 / 1$ \\
\hline Energieinhalt Batterie & 162 kWh & 162 kWh & 175 kWh & 189 kWh \\
\hline Ladeleistung & $200 \mathrm{~kW}$ & $200 \mathrm{~kW}$ & $200 \mathrm{~kW}$ & $200 \mathrm{~kW}$ \\
\hline $\begin{array}{l}\text { zu installierende Leistung } \\
\text { am Netzanschlusspunkt }\end{array}$ & $223 \mathrm{~kW}$ & $223 \mathrm{~kW}$ & $223 \mathrm{~kW}$ & $223 \mathrm{~kW}$ \\
\hline \multirow[t]{2}{*}{ Systemkosten } & $\begin{array}{l}\text { 7,314 Mio. } \\
\text { EUR }\end{array}$ & $\begin{array}{l}\text { 7,233 Mio. } \\
\text { EUR }\end{array}$ & $\begin{array}{l}\text { 7,224 Mio. } \\
\text { EUR }\end{array}$ & $\begin{array}{l}\text { 7,220 Mio. } \\
\text { EUR }\end{array}$ \\
\hline & -- & $100 \%$ & $99,88 \%$ & $99,82 \%$ \\
\hline Lebensdauer Batterie & 5,3 Jahre & 5,75 Jahre & 6,23 Jahre & 6,67 Jahre \\
\hline
\end{tabular}

Abschließend wird das Ergebnis hinsichtlich des maximalen und mittleren Optimierungspotentials bewertet. Dafür sind in Tabelle 5-17 die Systemkosten der optimalen Systemkonfiguration mit denen der teuersten Konfiguration sowie dem Mittelwert aller gültigen Systemkonfigurationen vergleichend dargestellt.

Tabelle 5-17: Optimierungspotential des erweiterten Szenarios

\begin{tabular}{ccc}
\hline optimale Konfiguration & teuerste Konfiguration & $\begin{array}{c}\text { Mittelwert aller } \\
\text { Konfigurationen }\end{array}$ \\
\hline 7,233 Mio. EUR & 8,386 Mio. EUR & 7,646 Mio. EUR \\
-- & $\frac{7,233 \text { Mio EUR }}{8,386 \text { Mio EUR }}=86,3 \%$ & $\frac{7,233 \text { Mio EUR }}{7,646 \text { Mio EUR }}=94,6 \%$ \\
\hline
\end{tabular}

Aus dem Verhältnis der Systemkosten der optimalen sowie der teuersten Konfiguration ergibt sich ein maximales Optimierungspotential von 13,7\%, welches im hier dargestellten Berechnungsbeispiel des erweiterten Szenarios erreicht werden kann. Der Mittelwert der Systemkosten aller technisch realisierbaren Konfigurationen beträgt 7,646 Mio. EUR. Im Verhältnis zur optimalen Konfiguration ergibt sich ein mittleres Optimierungspotential von $5,4 \%$. 


\subsection{Sensitivitätsanalyse betrieblicher und ökonomischer Parameter}

In diesem Abschnitt wird untersucht, inwiefern eine Veränderung der betrieblichen und ökonomischen Rahmenbedingungen Auswirkungen auf die optimale Systemkonfiguration haben. Die Untersuchungen in diesem Abschnitt basieren auf den Randbedingungen und Ergebnissen der Optimierungsrechnung des erweiterten Szenarios aus Kapitel 5.3.2.

\subsubsection{Einfluss des Fahrplantaktes auf die optimale Systemkonfiguration}

In Kapitel 3.1 wurde eine Änderung der Umlaufzeiten ausgeschlossen und damit die verfügbaren Ladezeiten als Randbedingungen fest definiert. Aus betrieblicher Sicht wird untersucht, ob eine Veränderung des Taktes eine Auswirkung auf das Optimum der Systemkonfiguration hat. Im Rahmen dieser Arbeit gilt die Annahme, dass eine Verdichtung oder Entzerrung des Taktes keinen Einfluss auf die Umlaufzeiten und somit auf das Betriebsprogramm hat. Es wird dadurch lediglich die Anzahl der notwendigen Fahrzeuge beeinflusst. Diese berechnet sich gemäß Formel (4-38).

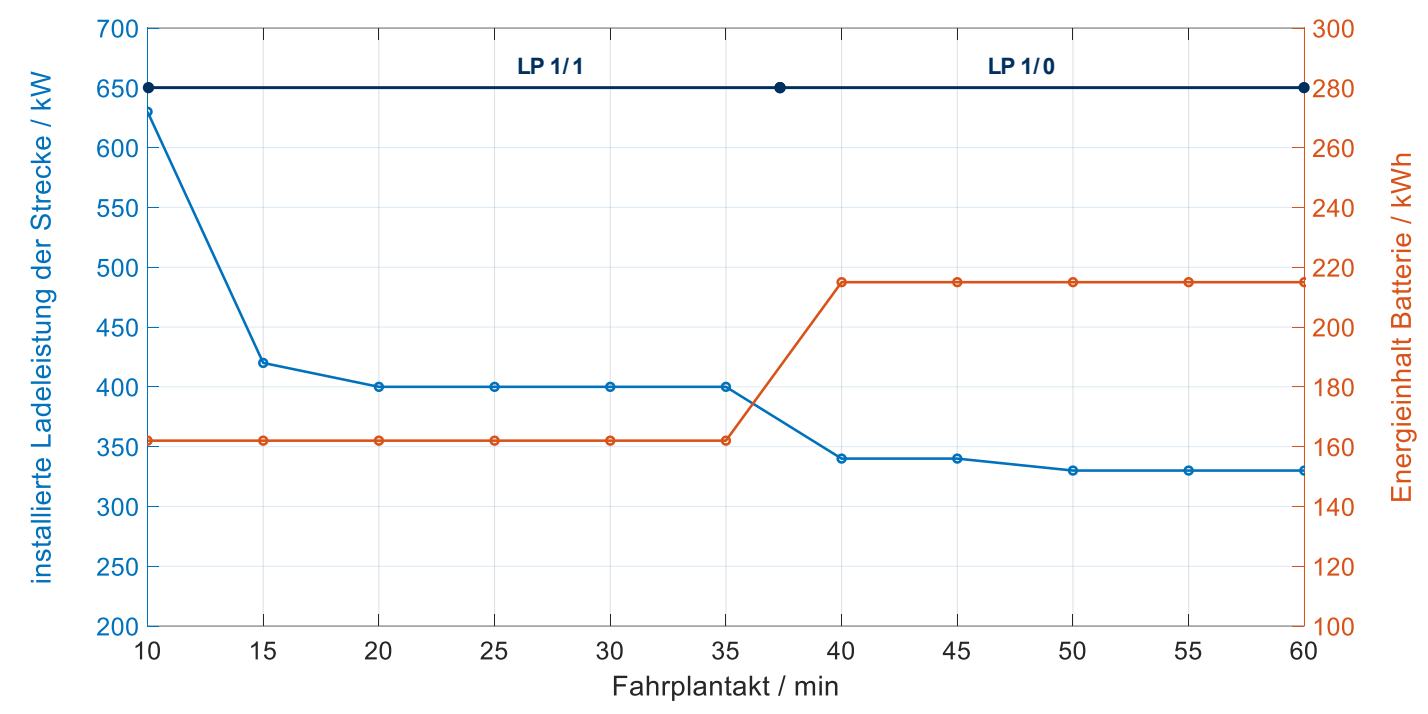

Abbildung 5-38: Optimale Systemkonfiguration als Funktion des Fahrplantaktes

Dem erweiterten Szenario liegt ein Fahrplan von 20 min zugrunde. Dieser wird nun zwischen 10 min und 60 min variiert. Die Ergebnisse der Variation sind in Abbildung 5-38 gegeben. Darin sind die jeweils zu installierende Nachladeleistung der Strecke (Summe aller LP) und der Energieinhalt der Batterie über der Variation des Fahrplantaktes dargestellt. Grundsätzlich ist ein gegenläufiger Verlauf der beiden Kurven Ladeleistung und Energieinhalt erkennbar. Bei einem sehr dichten Takt (entspricht einer hochbelasteten Strecke) führt eine hohe installierte Ladeleistung bei einer eher 
kleineren Batterie zum Systemkostenoptimum. Dagegen weisen schwächere Taktzeiten eine eher geringe Ladeleistung mit größerem Batterieenergieinhalt als Kostenoptimum auf. Im konkreten, hier betrachteten Anwendungsfall, beträgt der Energieinhalt der Batterie zwischen den Taktzeiten 10 min und 35 min jeweils $162 \mathrm{kWh}$. Anschließend folgt ein Sprung auf 215 kWh, was einer Erhöhung auf 133\% entspricht. Die installierte Ladeleistung der Strecke beträgt für schwache Taktzeiten $330 \mathrm{~kW}$. Mit dichter werdendem Takt steigt diese auf bis zu $420 \mathrm{~kW}$ bei einem 15 min Takt an. Aus Abbildung 5-38 geht hervor, dass zwischen den Taktzeiten $35 \mathrm{~min}$ und $40 \mathrm{~min}$ ein Wechsel der Ladepunktkombination auftritt. Während bei LP $1 / 0$ die volle Leistung in einem Ladepunkt installiert werden muss, wird diese bei LP $1 / 1$ auf zwei Ladepunkte aufgeteilt. Die Übergangsstelle ist durch den Anstieg des Energieinhalts der Batterie und die Reduzierung der installierten Leistung gekennzeichnet.

Auffällig ist der starke Anstieg der zu installierenden Ladeleistung bei einem $10 \mathrm{~min}$ Takt. Dies ist über die verfügbare Ladezeit am Ladepunkt 1 begründbar. Hier stehen 15 min Ladezeit zur Verfügung. Bei Realisierung eines 10 min Taktes stehen für 5 min zwei Fahrzeuge parallel am Ladepunkt. Zur Sicherstellung der Nachladung muss dieser Ladepunkt entsprechend doppelt ausgeführt werden. Dies führt in der abschließenden Bewertung dazu, dass die zu installierende Ladeleistung dreimal (einmal an LP 1 und zweimal an LP 2) realisiert werden muss.

\subsubsection{Sensitivitätsanalyse der einzelnen Kostenbestandteile}

Die optimalen Systemkonfigurationen, welche in den Optimierungsrechnungen ermittelt wurden, basieren auf den in dieser Arbeit beispielhaft angenommenen Kostensätzen (Vgl. Abbildung 5-26). Generell unterliegen Kosten und Preise jedoch Schwankungen, welche beispielsweise marktwirtschaftlich oder auch regulatorisch bedingt sein können. In diesem Abschnitt wird deshalb untersucht, inwiefern eine Variation der Kostensätze einen Einfluss auf die resultierenden Systemkosten hat. Dafür wird eine globale Sensitivitätsanalyse der Kostenparameter durchgeführt. Im Gegensatz zu einer lokalen Sensitivitätsanalyse wird dabei die Variation der Einflussfaktoren über ihren kompletten Definitionsbereich betrachtet (Siebertz et al., 2010).

Zur Ermittlung der Sensitivitäten werden die Kostenparameter zwischen 50\% und 150\% des ursprünglich parametrierten Wertes aus Abbildung 5-26 variiert und die daraus resultierenden Systemkosten ermittelt. Um eine Überlagerung von Effekten auszuschließen, wird jeweils nur ein Kostenparameter gleichzeitig variiert. Die resultierenden Systemkosten basieren stets auf der sich ergebenden optimalen Systemkonfiguration.

Die Ergebnisse der Sensitivitätsanalyse sind in Abbildung 5-39 gegeben. Daraus geht hervor, dass sich für alle Parameter ein nahezu linearer Verlauf ergibt. Dies ist über die 
jeweils optimale Systemkonfiguration begründbar, welche nahezu unverändert bleibt. Die Ladeleistung variiert zwischen 200 kW und 210 kW, während der Batterieenergieinhalt sowie die Ladepunktkombination über alle Kostenvariationen hinweg konstant bei $162 \mathrm{kWh}$ bzw. LP 1/1 bleiben. Eine Auflistung der optimalen Konfigurationen aller Berechnungen zur Variation der Kostenparameter ist in Anhang C.1 gegeben.

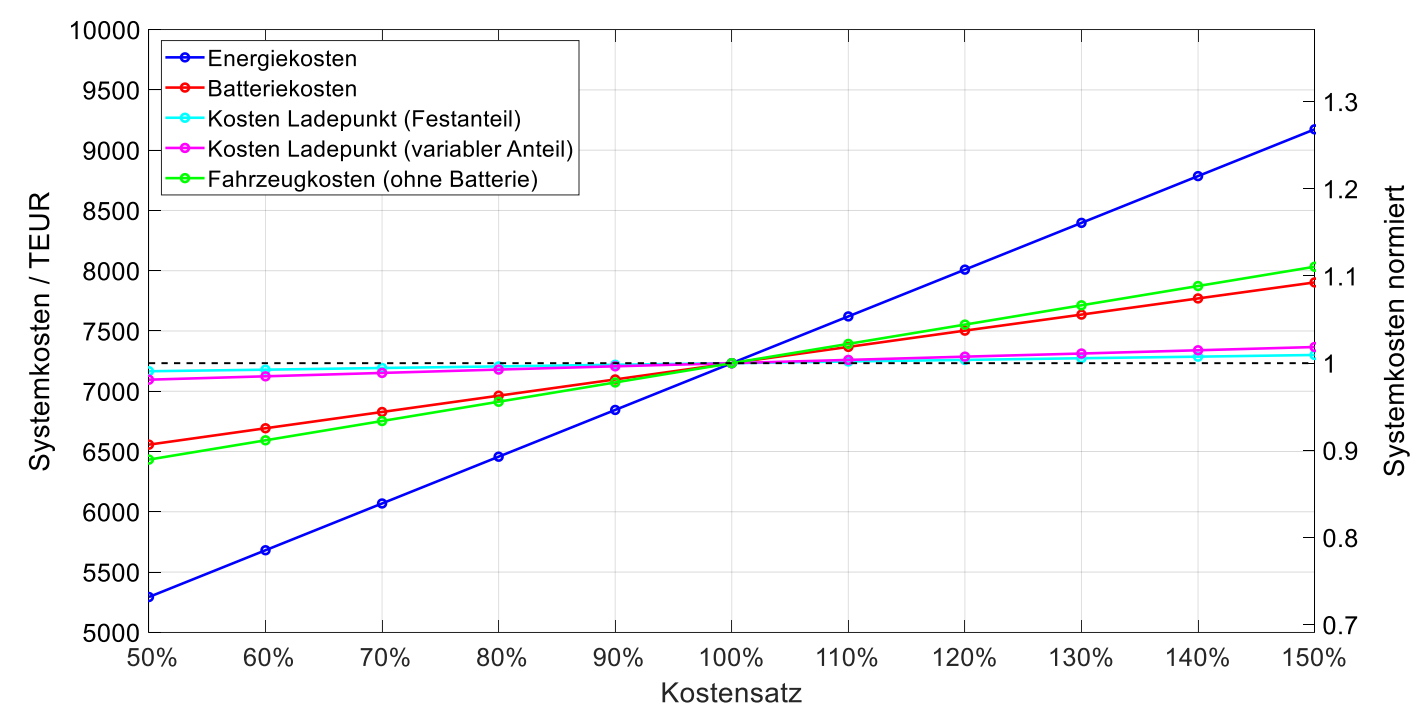

Abbildung 5-39: Sensitivität der Kostenbestandteile

Aus Abbildung 5-39 geht hervor, dass eine Änderung der Energiekosten die resultierenden Systemkosten am stärksten beeinflusst. Eine Halbierung der Energiekosten würde beispielsweise eine Reduktion der Systemkosten um 27\% bewirken. Dieser Zusammenhang ist vor allem deshalb interessant, da Elektrobusse zum gegenwärtigen Zeitpunkt die gesetzliche EEG-Umlage in voller Höhe zahlen müssen, während beispielsweise für Schienenbahnen eine um $80 \%$ reduzierte Umlage ${ }^{20}$ zu entrichten ist (VDV, 2016; EMCEL, 2017). Im Jahr 2019 beträgt die EEG-Umlage 6,405 ct/kWh (BNetzA, 2019). Bei einer Reduzierung um $80 \%$ würde diese noch $1,28 \mathrm{ct} / \mathrm{kWh}$ betragen. Die Auswirkungen auf die Systemkosten sind in Tabelle 5-18 dargestellt. Daraus geht hervor, dass mit einer reduzierten EEG-Umlage die Systemkosten im hier gezeigten Beispiel um ca. $14 \%$ gesenkt werden könnten.

Neben den Energiekosten weisen auch die übrigen Kostenbestandteile deutlich geringere Sensitivitäten auf. Aus Abbildung 5-39 geht hervor, dass die Batteriekosten und die Fahrzeugkosten eine annähernd gleich große Sensitivität auf die Systemkosten haben. Beispielsweise würde eine Halbierung der Batteriekosten eine Reduzierung der Systemkosten um 10\% bewirken. Dagegen haben die Kosten der Ladeinfrastruktur

\footnotetext{
${ }^{20}$ Nach $\S 65$ EEG ist eine Reduzierung um 80\% für Schienenbahnen dann möglich, wenn der jährliche Verbrauch mindestens $2 \mathrm{GWh}$ beträgt.
} 
kaum einen Einfluss auf die Entwicklung der Systemkosten. Eine Halbierung der variablen Ladepunktkosten würde beispielsweise nur zu einer Reduzierung der Systemkosten von $2 \%$ führen.

Tabelle 5-18: Berechnungsbeispiel EEG-Umlage

\begin{tabular}{ll}
\hline Parametrierte Energiekosten & $20 \mathrm{ct} / \mathrm{kWh}$ \\
EEG-Umlage 2019 & $6,405 \mathrm{ct} / \mathrm{kWh}$ \\
Reduzierte EEG-Umlage (um 80\%) & $1,28 \mathrm{ct} / \mathrm{kWh}$ \\
Neue Energiekosten & $14,88 \mathrm{ct} / \mathrm{kWh}(74,4 \%)$ \\
Neue Systemkosten & $6240 \mathrm{TEUR}(86,3 \%)$ \\
\hline
\end{tabular}

Aus den Ergebnissen dieses Abschnitts geht hervor, dass die Energiekosten aus wirtschaftlicher Sicht den größten Stellhebel bei der Systemauslegung einer Elektrobuslinie darstellen. Neben der regulatorischen Möglichkeit einer Reduzierung der EEG-Umlage für Elektrobusse ist es für ein Verkehrsunternehmen empfehlenswert, einen möglichst günstigen Stromtarif mit dem lokalen Energieversorger zu verhandeln. Des Weiteren wird die Erwartung nach einer Kostenreduzierung des Gesamtsystems oftmals mit der Hoffnung auf sinkende Batteriepreise verbunden. In (Bloomberg, 2018) wird eine Reduzierung der Preise für Elektrobusbatteriepacks um mehr als $80 \%$ bis 2030 gegenüber dem Jahr 2016 erwartet. Es wurde jedoch gezeigt, dass bereits mit einer Halbierung der Batteriekosten die Systemkosten mit ca. 10\% nur moderat reduziert werden können. Zusätzlich kann festgehalten werden, dass die Kosten der Ladeinfrastruktur entlang der Strecke einen sehr kleinen Anteil an den Gesamtkosten haben und darüber hinaus nur eine sehr geringe Sensitivität bezüglich der Kostenänderungen aufweisen. 


\section{Zusammenfassung, Fazit und Ausblick}

\subsection{Zusammenfassung und Fazit der Arbeit}

Die Einführung von Elektrobussen als Ersatz konventioneller Dieselbusse in kommunalen Verkehrsunternehmen ist eine wichtige und aktuelle Maßnahme vieler deutscher und europäischer Städte zur Reduzierung von Emissionen und zur Einhaltung gesetzlicher Grenzwerte. Im Gegensatz zu Dieselbussen, welche eine große Reichweite aufgrund der hohen Energiedichte des Kraftstoffs vorweisen, müssen Elektrobusse stets als System aufgefasst werden, bei welchem die Ladeinfrastruktur eine betriebsrelevante Teilkomponente darstellt. Die Einführung von Elektrobussen ist aktuell mit hohen finanziellen Aufwänden verbunden. Die Übernahme eines Teils der investiven Mehrkosten gegenüber Dieselbussen ist derzeit Gegenstand verschiedener nationaler und europäischer Förderprogramme. Ziel dieser Arbeit war die Entwicklung einer Methodik, welche eine Reduzierung der elektrobusspezifischen Mehrkosten ermöglicht und damit eine wirtschaftliche Ausgestaltung des Gesamtsystems sicherstellt.

Als Ergebnis der Literaturrecherche wurde der Fokus dieser Arbeit auf die Entwicklung einer Simulationsumgebung zur Systemsimulation gelegt. Dies beinhaltet insbesondere eine detaillierte, ganzheitliche Simulation der Fahrzeug-Traktionsbatterie inkl. verschiedener Nachladeszenarien. Dafür wurde ein zweistufiger Simulationsansatz mit unterschiedlichen Zeitebenen entwickelt. Die elektro-thermische Belastung wird in Mikrozeitschritten berechnet, die anschließende Berechnung der resultierenden Batteriealterung findet auf Basis von Makrozeitschritten statt. Während die elektrische und thermische Modellierung der Batterie auf teilweise aus der Literatur bekannten Verfahren aufbaut, wurde ein empirisches Alterungsmodell entwickelt. Neben dem Modellaufbau und der Parametrierung stand vor allem die Definition eines Prozesses zur Berechnung realer Fahrszenarien mit dynamischen Stromprofilen im Vordergrund. Das Alterungsmodell sowie der Berechnungsprozess wurden anhand zweier Messreihen validiert. Des Weiteren wurde eine Optimierungsfunktion entwickelt, welche ein integraler Bestandteil der Simulationsumgebung ist. Die Optimierung basiert auf einer Variation wichtiger Systemparameter. Im Rahmen dieser Arbeit sind dies der Energieinhalt der Batterie, Anzahl und Ort der Nachladepunkte und die Ladeleistung. Eine konkrete Kombination dieser Variationsparameter wird als Systemkonfiguration bezeichnet. Die integrierte Optimierungsfunktion ermöglicht eine automatisierte Berechnung einer Vielzahl solcher Konfigurationen, die anhand eines Kostenmodells bewertet und somit vergleichbar gemacht werden. Die optimale Systemkonfiguration für eine konkrete Buslinie ist bei minimalen Systemkosten gegeben. 
Mittels der entwickelten Systemsimulationsumgebung wurden verschiedene Einzelsimulationen und Optimierungsrechnungen einer Elektrobuslinie durchgeführt. Für die betrachtete Buslinie können folgende Aussagen abgeleitet werden:

- Die elektro-thermische Belastung der Batterie wird hauptsächlich durch die konkrete Wahl der Systemkonfiguration sowie betriebliche Aspekte wie Verspätungen bestimmt. Die maßgebliche Größe zur Beurteilung ist der SOC-Hub ${ }^{21}$. Aus Tabelle 5-9 in Kapitel 5.2.1 geht hervor, dass der SOC-Hub die Lebensdauer der Batterie wesentlich beeinflusst. Sowohl die Abnahme der Kapazität als auch der Anstieg des Innenwiderstands nehmen mit steigendem SOC-Hub überproportional zu.

- In der Systemoptimierung führen stets vergleichsweise kleine Batterien zum wirtschaftlichen Optimum. In den Beispielen dieser Arbeit wurde stets ein Batterieenergieinhalt als optimal ermittelt, welcher nur geringfügig über dem erforderlichen Minimum zur Bewältigung eines einzelnen Umlaufs liegt. Die Kosten der Batterien stellen einen wesentlichen Anteil an den Gesamtkosten dar, da in vielen Anwendungsfällen ein Batterietausch im Laufe des Fahrzeuglebenszyklus notwendig ist.

- Aus dem vorherigen Punkt kann abgeleitet werden, dass für eine kostenoptimale Linienauslegung stets angepasste Batteriegrößen für den jeweiligen Anwendungsfall ermittelt werden. Daraus resultiert, dass Elektrobusse gegenüber Dieselbussen eine deutlich eingeschränkte Nutzbarkeit im täglichen Betrieb haben, da einzelne Linien stark unterschiedliche Streckencharakteristiken sowie Einsatzprofile und Laufleistungen aufweisen können. Der vielfach vorgetragene Wunsch von Verkehrsunternehmen, Elektrobusse möglichst genauso flexibel einsetzen zu können wie Dieselbusse, kann mit dem derzeitigen Stand der (Batterie-)Technik eher nicht erfüllt werden, da dies eine Beschaffung von Fahrzeugen nach der Linie mit der größten Beanspruchung bedeuten würde. Dabei würden die Fahrzeuge für übrige Linien zum Teil deutlich überdimensioniert, was wiederum einen erheblichen Kostentreiber darstellt.

- Die Nachladestrategie des Speichers bestimmt über den SOC-Hub maßgeblich dessen Alterung und hat somit einen erheblichen Anteil an den Batteriekosten während des Fahrzeuglebenszyklus. Aus den Ergebnissen der Arbeit kann geschlussfolgert werden, dass grundsätzlich ein ladungserhaltender SOCVerlauf mit möglichst kleinen SOC-Hüben während des Tages zu einer minimalen Alterung führt. Das bedeutet, dass bei einer Nachladung stets der max.

\footnotetext{
${ }^{21}$ Diese Aussage gilt unter der Annahme, dass die Batterietemperatur durch eine ausreichende Kühlung oder Heizung innerhalb des vom Hersteller definierten Betriebsbereichs gehalten wird.
} 
SOC-Wert erreicht wird. Dies gilt sowohl für eine neue als auch für eine gealterte Batterie mit reduzierter Kapazität und erhöhtem Innenwiderstand.

- Die Sicherstellung eines ladungserhaltenden SOC-Verlaufs muss über eine geeignete Wahl der Nachladestrategie und demnach der Ladeinfrastruktur unter Beachtung der verfügbaren Ladezeit und eines pünktlichen Betriebsablaufs realisiert werden. Grundsätzlich kann die Aussage getroffen werden, dass die Batterie dafür möglichst oft während des Tages nachgeladen werden muss.

- Bei der Suche nach einer wirtschaftlich optimalen Systemkonfiguration entstehen teilweise konkurrierende Optimierungsziele. Dieses mehrkriterielle Optimierungsproblem kann mittels Betrachtung der Systemkosten auf ein einzelnes Optimierungsziel vereinfacht werden. Die integrierte Optimierungsfunktion ermöglicht eine Berechnung aller möglichen Kombinationen der Systemparameter innerhalb des definierten Wertebereiches. Somit wird sichergestellt, dass alle technisch realisierbaren Systemkonfigurationen in die Bewertung einbezogen werden. Gegenüber einem heuristischen Ansatz wie in Tabelle 2-4 kann sichergestellt werden, dass die jeweils optimale Systemkonfiguration tatsächlich gefunden wird.

- Energiekosten haben die größte Sensitivität auf die resultierenden Systemkosten und bieten demnach den größten Stellhebel zur Kostenreduktion. Neben regulatorischen Aspekten wie der EEG-Umlage kann der lokale Energieversorger über einen günstigen Stromtarif einen entscheidenden Beitrag zur Kostenreduzierung leisten.

- Die notwendige Ladeinfrastruktur entlang der Strecke hat nur einen sehr geringen Einfluss auf die resultierenden Systemkosten. Darüber hinaus tragen geringere Infrastrukturkosten nur in geringem Maße zur Reduzierung der Gesamtsystemkosten bei.

- Für eine konkrete Linie mit einem vorgegebenen Fahrplantakt führt eine bestimmte Kombination aus Batterieenergieinhalt und Ladeinfrastruktur zum wirtschaftlichen Optimum. Anhand einer Sensitivitätsanalyse wurde gezeigt, dass die Wirtschaftlichkeit der Ladeinfrastruktur eine Abhängigkeit vom Fahrplantakt aufweist. Mit dichter werdendem Takt verlagert sich das Verhältnis aus Energieinhalt der Batterie und installierter Ladeleistung eindeutig in Richtung der Ladeinfrastruktur.

Insgesamt konnte gezeigt werden, dass die elektrobusspezifischen Mehrkosten durch ein effizientes Systemdesign reduziert werden können. Ein wirtschaftliches Optimum kann vor allem aufgrund der limitierten Leistungsfähigkeit der Batterie meist nur für eine bestimmte Linie ermittelt werden. Die Funktionalität der entwickelten Systemsimulation wurde anhand praxisnaher Beispiele gezeigt. Aus den Ergebnissen konnten anwendungsrelevante Aussagen und Parametersensitivitäten abgeleitet werden. 


\section{Kritische Auseinandersetzung}

Im Folgenden werden einige Aspekte der Arbeit behandelt, welche aus Sicht des Autors als verbesserungswürdig einzustufen sind und in künftigen Forschungsarbeiten adressiert werden könnten.

Die Modellierung der Batterie könnte punktuell detailliert werden. Im elektrischen Modell wurden die Parameter des RC-Glieds als konstant angenommen. Eine Erweiterung um eine Temperatur- und SOC-Abhängigkeit würde die Güte des Modells weiter verbessern.

Für die thermische Modellierung könnten neben der Anordnung in x-Richtung weitere Anordnungsformen der Zellen innerhalb eines Moduls berücksichtigt werden, beispielsweise in y-Richtung. Zudem ist die Annahme, dass zwischen einzelnen Modulen keine thermischen Beeinflussungen vorliegen, kritisch zu prüfen. Des Weiteren konnten die Kühlstrategien der forcierten Luftkühlung und der Wasserkühlung aufgrund fehlender Test- und Messtechnik nicht validiert werden.

Das Alterungsmodell weist von allen drei Teilmodellen die größten Abweichungen auf. Aufgrund des sehr langen Messzeitraums von über 1,5 Jahren war es nur schwer möglich, stets konstante Mess- und Umgebungsbedingungen zu garantieren. Außerdem konnte jeder Arbeitspunkt zur Parametrierung nur jeweils einmal angefahren werden, sodass keine statistische Sicherheit besteht. An dieser Stelle sollte ein erneuter Parametrierungs- und Validierungsdurchlauf mit Zellen einer neueren Generation vorbereitet und durchgeführt werden.

Die Modellierung der Ladeinfrastruktur berücksichtigt derzeit nur einen konstanten Wirkungsgrad, bei welchem alle elektrischen und elektronischen Komponenten zusammengefasst werden. Vor allem Transformatoren und Leistungshalbleiter weisen jedoch oftmals einen nichtkonstanten und teilweise nichtlinearen Wirkungsgradverlauf auf, wobei hauptsächlich der Teillastbereich von Interesse ist. Des Weiteren wurde im Rahmen dieser Arbeit keine Rückwirkung auf das speisende Energienetz betrachtet. Dies ist jedoch besonders dann notwendig, wenn bei Flottenumstellungen eine große Anzahl an Fahrzeugen innerhalb kurzer Zeit geladen werden sollen.

Die Ladeinfrastruktur im Betriebshof wurde in dieser Arbeit nicht berücksichtigt. Bei der Elektrifizierung von Busdepots stehen die Verkehrsunternehmen jedoch vor erheblichen Herausforderungen. Neben der räumlichen Installation der Ladeinfrastruktur sind vor allem die intelligente und automatisierbare Steuerung der Nachladung zur Reduzierung von Leistungsspitzen sowie die softwareseitige Integration der Fahrzeuge in bestehende Betriebshofmanagementsysteme von großer Bedeutung. Auch hier ist die Rückwirkung der Nachladung auf das speisende Energienetz ein kritischer Punkt, welcher im Dimensionierungsprozess berücksichtigt werden muss. 
In dieser Arbeit wurde eine Betrachtung einzelner Linien fokussiert, da nach dem derzeitigen Stand viele Verkehrsunternehmen vor Teilumstellung ihrer Flotten stehen. Mit fortschreitender Zeit werden jedoch zunehmend vollständige Flottenumstellungen innerhalb kürzerer Zeiträume erwartet. Eine Erweiterung der Simulationsumgebung hin zur Betrachtung, Berechnung und Optimierung ganzer Busnetze erscheint angesichts dieser Entwicklung als erstrebenswert. Die damit steigende Komplexität des Berechnungsprozesses sollte jedoch nicht zu Lasten einer vereinfachten Batteriesimulation gehen, da die Batterie auch in absehbarer Zeit die sensibelste und teuerste Einzelkomponente eines Elektrobusses darstellen wird.

\subsection{Ausblick}

Die Simulationsumgebung mit integrierter Optimierungsfunktion kann im tatsächlichen Umstellungsprozess eines Verkehrsunternehmens angewendet werden. Hierbei ist besonders wichtig, dass die Parametrierung des Batteriemodells möglichst nah an den Einzelzellen potentieller Systemanbieter liegt. Es wurde gezeigt, dass eine allgemeingültige Parametrierung einer Traktionsbatterie aufgrund der großen Variationsbreite nicht zum gewünschten Ergebnis führt.

Im konkreten Anwendungsfall wird es zudem darauf ankommen, dass die in der Systemsimulation ermittelte optimale Konfiguration auch in exakt dieser Form umsetzbar ist. Vor allem der Energieinhalt der Batterie ist häufig in größeren Schrittweiten (als in dieser Arbeit angenommen) verfügbar. Gleiches betrifft die Leistung von Ladepunkten. Hersteller bieten oftmals nicht beliebig skalierbare Geräte und Systeme an, welche stattdessen nur wenige Leistungsabstufungen ermöglichen. Die Herausforderung besteht darin, die wissenschaftlichen Ergebnisse in eine ausführbare Anwendung zu überführen. Dafür kann es unter Umständen notwendig sein, eine erneute Systemoptimierung mit einem eingeschränkten Parameterraum durchzuführen.

Des Weiteren gilt es, regulatorische und praktische Hürden in der Planung und der Errichtung des Systems zu kalkulieren. Vor allem die Errichtung von Ladeinfrastruktur im öffentlichen Raum kann sich dabei als langwierig erweisen. Beispielsweise musste in Dresden ein funktionsfähiger Ladepunkt außer Betrieb genommen werden, da die notwendige Freigabe durch die technische Aufsichtsbehörde aufgrund einer fehlenden Dokumentation nicht erteilt werden konnte (DNN, 2017).

Ein elementarer Bestandteil der als optimal ermittelten Systemkonfiguration ist die Einhaltung der dazugehörigen Nachladestrategie. Beispielsweise wurde im Rahmen des Projektes "Elektrobus Linie 79" die Entscheidung über eine Nachladung am Wendepunkt dem Fahrer überlassen. Es wurde lediglich eine unverbindliche Empfehlung zur Nachladung mittels eines CAN-Displays angezeigt. Dies führte zu einer großen Variation an SOC-Verläufen (Bunzel et al., 2016). Als Konsequenz ist in der praktischen 
Umsetzung darauf zu achten, dass die in der Systemauslegung ermittelten Nachladevorgänge im regulären Betrieb auch entsprechend umgesetzt werden. Diese sollten für Fahrer obligatorisch sein und keinen Freiheitsgrad darstellen. Mit Blick auf die Zukunft könnte dieses Thema ohnehin obsolet werden, da der Forschungsbereich des automatisierten Fahrens zunehmend den Bereich ÖPNV adressiert. Bei hochautomatisierten Bussystemen wird die Durchführung der Nachladung ein Bestandteil der Automatisierung sein.

Grundsätzlich stellt sich die Frage, ob das Verkehrsmittel Stadtbus auch zukünftig noch Teil der Lösung zur Befriedigung der Mobilitätsbedürfnisse der Menschen, vor allem in immer größeren und dichteren Städten, sein wird. Die zunehmenden Diskussionen um On-Demand-Anbieter, automatisierte Flugtaxis und Shuttles oder aufgeständerte Monorails zeigen, dass die urbane Mobilität vor großen Herausforderungen und Umbrüchen steht. 


\section{Literatur}

A123 Systems (2011), Nanophosphate ${ }^{\circledR}$ Lithium Ion Prismatic Pouch Cell AMP20M1HD-A: Zelldatenblatt.

A123 Systems (2014), Battery Pack Design, Validation, and Assembly Guide using A123 Systems Nanophosphate ${ }^{\circledR}$ Cells: User Documentation.

Akasol AG (2019), High Performance Battery Systems for Buses. Information zur Anwendung in Bussen (abgerufen 31.10.2019).

Baumhöfer, T., Brühl, M., Rothgang, S., Sauer, D.U. (2014), Production caused variation in capacity aging trend and correlation to initial cell performance, Journal of Power Sources, Vol. 247, S. 332-338.

Berthold, K. (2019), Techno-ökonomische Auslegungsmethodik für die Elektrifizierung urbaner Busnetze, Dissertation, Karlsruher Institut für Technologie, Karlsruhe, 2019.

Bloomberg (2018), Electric Buses in Cities: Driving Towards Cleaner Air and Lower $\mathrm{CO} 2$.

BMVI (2016), Statusbericht 2015/16 Hybrid- und Elektrobus-Projekte in Deutschland, Berlin.

BNetzA (2019), EEG-Umlage. Was ist die EEG-Umlage und wie funktioniert sie?, unter: https://www.bundesnetzagentur.de/SharedDocs/FAQs/DE/Sachgebiete/Energie/V erbraucher/Energielexikon/EEGUmlage.html (abgerufen 31.07.2019).

Boettcher, J. (2019), Automatisierte Erkennung und Auswertung verkehrlicher Sonderstellen in ÖPNV-Stadtbus-Trajektorien, Diplomarbeit, Technische Universität Dresden, Dresden, 2019.

Büchner, S. (2015), Ladestationen für Elektrobusse: Flexibel und Sicher, 3. Dresdner Fachkonferenz - Innovation Elektrobus, Dresden.

Buller, S. (2002), Impedance-based simulation models for energy storage devices in advanced automotive power systems, Dissertation, RWTH Aachen, Aachen, 2002.

Bunzel, A., Bäker, B. (2018), Energy consumption of electric city buses Determination as a part of a technological and economic evaluation of bus lines with regards to their electrifiability, in IEEE (Hrsg.), 2018 IEEE International Conference on Electrical 
Systems for Aircraft, Railway, Ship Propulsion and Road Vehicles \& International Transportation Electrification Conference (ESARS-ITEC), S. 1-5.

Bunzel, A., Petersohn, R., Bäker, B. (2016), Dresden's bus route 79 turned into full electric - Opportunity and challenge, in Hoff, C., Sirch, O. (Hrsg.), Elektrik, Elektronik in Hybrid- und Elektrofahrzeugen und elektrisches Energiemanagement VII, Fachbuch / Haus der Technik, expert Verlag, Renningen.

Corneille, M. (2018), E-ÖPNV: Technologie und Einführungsstrategie, DVWG aktuell, Vol. 46, S. 7-8.

DNN (2017), E-Bus der Dresdner Verkehrsbetriebe mit "Anfangsschwierigkeiten", unter: https://www.dnn.de/Dresden/Lokales/E-Bus-der-DresdnerVerkehrsbetriebe-mit-Anfangsschwierigkeiten (abgerufen 05.08.2019).

Doering, E., Schedwill, H., Dehli, M. (2016), Grundlagen der Technischen Thermodynamik: Lehrbuch für Studierende der Ingenieurwissenschaften, 8., überarbeitete und erweiterte Auflage, Springer Vieweg, Wiesbaden.

DVB (2019), Das Unternehmen: Zahlen, Daten und Fakten im Überblick - Stand: 31. Dezember 2018, Dresden.

Einhorn, M., Conte, V., Kral, C., Fleig, J. (2011), Comparison of electrical battery models using a numerically optimized parameterization method, in IEEE (Hrsg.), IEEE Vehicle Power and Propulsion Conference (VPPC), 2011, Chicago, IL, USA, 06.09.2011 - 09.09.2011, IEEE, Piscataway, NJ, S. 1-7.

EMCEL (2017), Was bringt die Ermäßigung der Stromsteuer für Elektrobusse?, unter: https://emcel.com/de/stromsteuer-fuer-elektrobusse/ (abgerufen 31.07.2019).

Farhat, F. (2018), Überführung realer Lastprofile in Laborzyklen zur Untersuchung von Alterungsvorgängen elektrochemischer Speicher, Forschungspraktikum, Technische Universität Dresden, Dresden, 2018.

Fichtl, H., Steinert, F. (2016), IVIsion and IVInet - Tool Chain for the Electrification of City Bus Routes, Transportation Research Procedia, Vol. 14, S. 2554-2563.

Fischhaber, S., Gassmann, G., Schuster, S.F. (2016), Studie: Second-Life-Konzepte für Lithium-Ionen-Batterien aus Elektrofahrzeugen: Analyse von Nachnutzungsanwendungen, ökonomischen und ökologischen Potenzialen Schaufenster Elektromobilität - eine Initiative der Bundesregierung, Ergebnispapier der Begleitund Wirkungsforschung / Deutsches Dialog Institut GmbH, Frankfurt am Main.

Fleckenstein, M. (2013), Modellbasiertes Thermomanagement für Li-lonen-Zellen in elektrischen Fahrzeuganwendungen, Dissertation, Technische Universität Dresden, Dresden, 2013. 
Freitag, A. (2018a), Entwicklung eines Lademanagementsystems für die Depotladung von Elektrobussen, Forschungspraktikum, Technische Universität Dresden, Dresden, 2018.

Freitag, A. (2018b), Intelligentes Thermomanagement zur Vorkonditionierung der Batterie eines Elektrobusses, Diplomarbeit, Technische Universität Dresden, Dresden, 2018.

Gatter, J. (2019), Erstellung, Parametrierung und Validierung eines thermischen Modells für Li-lonen Traktionsbatterien, Forschungspraktikum, Technische Universität Dresden, Dresden, 2019.

Herb, F. (2010), Alterungsmechanismen in Lithium-Ionen-Batterien und PEMBrennstoffzellen und deren Einfluss auf die Eigenschaften von daraus bestehenden Hybrid-Systemen, Dissertation, Universität UIm, UIm, 2010.

Hipp, C. (2019), Thermomanagement für Batterien von Elektrobussen, 10. VDVKonferenz Elektrobusse - Markt der Zukunft!

Holz, V., Lonoce, L., Warner, B. (2017), Grid Compatible Flash Charging Technology: TOSA e-bus infrastructure, 1st E-Mobility Power System Integration Symposium, Berlin.

Jefferies, D., Göhlich, D. (2018), Integrated TCO Assessment of Bus Network Electrification Considering Rescheduling and Delays, in 31st International Electric Vehicle Symposium \& Exhibition \& International Electric Vehicle Conference (EVS31), Kobe, Japan, 30/09/2018 - 03/10/2018, EVS.

Jossen, A. (2016), Modellierung und Simulation moderner Batteriesysteme und hybrider Energiespeicher Topologien, Workshop Dezentrale Sektorkopplung und Hybride Energiespeichersysteme, Dresden.

Jossen, A., Weydanz, W. (2006), Moderne Akkumulatoren richtig einsetzen: 36 Tabellen, Ubooks, Neusäß.

Jossen, A., Weydanz, W. (2019), Moderne Akkumulatoren richtig einsetzen, 2. überarbeitete Auflage, Cuvillier Verlag, Göttingen.

Kampker, A., Vallée, D.,Schnettler, A. (Hrsg.) (2018), Elektromobilität: Grundlagen einer Zukunftstechnologie, 2. Auflage, Springer Vieweg, Berlin.

Knote, T., Haufe, B., Saroch, L. (2017), E-Bus-Standard: "Ansätze zur Standardisierung und Zielkosten für Elektrobusse", Fraunhofer-Institut für Verkehrs- und Infrastruktursysteme (IVI), Dresden. 
Kritzinger, F. (2018a), Erweiterung und Parametrierung eines thermischen Modells für Hochvolt-Lithium-Ionen-Energiespeicher, Forschungspraktikum, Technische Universität Dresden, Dresden, 2018.

Kritzinger, F. (2018b), Erweiterung und Parametrierung eines thermischen Modells für Hochvolt-Lithium-Ionen-Energiespeicher, Verteidigung Forschungspraktikum, Dresden.

Kritzinger, F. (2019), Modellierung des elektrothermischen Verhaltens eines Batteriemoduls mit Hilfe der elektrothermischen Impedanzspektroskopie, Diplomarbeit, Technische Universität Dresden, Dresden, 2019.

Kunith, A. (2017), Elektrifizierung des urbanen öffentlichen Busverkehrs. Technologiebewertung für den kosteneffizienten Betrieb emissionsfreier Bussysteme, Dissertation, Technische Universität, Berlin, 2017.

Kurzweil, P., Dietlmeier, O. (2018), Elektrochemische Speicher: Superkondensatoren, Batterien, Elektrolyse-Wasserstoff, Rechtliche Rahmenbedingungen, 2., aktualisierte und erweiterte Auflage, Springer Vieweg, Wiesbaden.

Lewerenz, M. (2018), Sezierung und quantitative Beschreibung der Alterung von LiIonen-Batterien mittels zerstörungsfreier Methoden validiert durch Post-MortemAnalysen, Disputationsvortrag, Aachen.

Liebers, M., Kloß, R., Bäker, B. (2016), Combined Power Train and Thermal Management Optimization Using an Extended Dynamic Programming, in Bargende, M., Reuss, H.-C., Wiedemann, J. (Hrsg.), 16. Internationales Stuttgarter Symposium, Springer Fachmedien, Wiesbaden, S. 367-381.

Meubrink, L. (2016), Optimierte Ladestrategien für ein batterieelektrisches Fahrzeug im ÖV, Diplomarbeit, Technische Universität Dresden, Dresden, 2016.

Neuendorff, M. (2018), Intelligente Ladestationen und Roboter-Busse, unter: https://www.moz.de/nachrichten/brandenburg/artikel-ansicht/dg/0/1/1650584 (abgerufen 08.07.2019).

Omar, N., Monem, M.A., Firouz, Y., Salminen, J., Smekens, J., Hegazy, O., Gaulous, H., Mulder, G., van den Bossche, P., Coosemans, T., van Mierlo, J. (2014), Lithium iron phosphate based battery - Assessment of the aging parameters and development of cycle life model, Applied Energy, Vol. 113, S. 1575-1585.

Paul, B.S. (2015), Analyse der Ausfallwahrscheinlichkeiten von Lithium-IonenEnergiespeichern in elektrifizierten Fahrzeugen, Dissertation, Universität Ulm, Ulm, 2015. 
Petersohn, R. (2012), Untersuchungen zur Alterung von Lithium-lonen-Zellen, Diplomarbeit, Technische Universität Dresden, Dresden, 2012.

Reif, K. (Hrsg.) (2012), Kraftfahrzeug-Hybridantriebe: Grundlagen, Komponenten, Systeme, Anwendungen, Vieweg+Teubner Verlag, Wiesbaden.

Richter, S. (2017), Weiterentwicklung eines thermisch-elektrischen Modells einer Lithium-Ionen-Zelle unter Einbeziehung von Alterungseffekten, Diplomarbeit, Technische Universität Dresden, Dresden, 2017.

Rogge, M., Sinhuber, P., Sauer, D.U. (2015a), Development of Cost-Efficient Electrification Strategies for Public Transport Bus Networks, in Bäker, B., Morawietz, L. (Hrsg.), Energy efficient vehicles 2015: Visions, trends and solutions for energy efficient vehicle systems, TUDpress, Dresden, S. 185-193.

Rogge, M., Wollny, S., Sauer, D.U. (2015b), Fast Charging Battery Buses for the Electrification of Urban Public Transport-A Feasibility Study Focusing on Charging Infrastructure and Energy Storage Requirements, Energies, Vol. 8 No. 5, S. 4587-4606.

Rudat, R. (2019), Fahrereinflussanalyse auf den Traktionsleistungsbedarf elektrifizierter Stadtbusse, Diplomarbeit, Technische Universität Dresden, Dresden, 2019.

Saidani, A. (2019), Weiterentwicklung eines Ladereglers für die Depotladung von Elektrobussen, Studienarbeit, Technische Universität Dresden, Dresden, 2019.

Schmalstieg, J., Käbitz, S., Ecker, M., Sauer, D.U. (2014), A holistic aging model for Li(NiMnCo)O2 based 18650 lithium-ion batteries, Journal of Power Sources, Vol. 257, S. 325-334.

Schuster, S.F., Bach, T., Fleder, E., Müller, J., Brand, M., Sextl, G., Jossen, A. (2015), Nonlinear aging characteristics of lithium-ion cells under different operational conditions, Journal of Energy Storage, Vol. 1, S. 44-53.

Schwarze, J. (2019), Die Umrüstung der KVB-Busflotte auf elektrische Antriebe, 10. VDV-Konferenz Elektrobusse - Markt der Zukunft!, Berlin.

Siebertz, K., van Bebber, D., Hochkirchen, T. (2010), Statistische Versuchsplanung: Design of Experiments (DoE), VDI-Buch, Springer, Heidelberg.

SILEO (2019), Technische Daten: Sileo S18 (abgerufen 17.01.2019). 
Steinert, F. (2015), Challenges of Pure Electric Driving in the Field of Public Transport. Energy Efficiency as Necessity for Usability, in Bäker, B., Morawietz, L. (Hrsg.), Energy efficient vehicles 2015: Visions, trends and solutions for energy efficient vehicle systems, TUDpress, Dresden, S. 177-184.

Thielmann, A., Sauer, A., Wietschel, M. (2015), Gesamt-Roadmap Energiespeicher für die Elektromobilität 2030, Karlsruhe.

Tschakert, W. (2014), Kein leichtes Spiel, Bus-Fahrt, Vol. 62 No. 10, 816-820.

Uebel, S. (2018), Eine im Hybridfahrzeug einsetzbare Energiemanagementstrategie mit effizienter Längsführung, Dissertation, Technische Universität Dresden, Dresden, 2018.

Ufert, M., Batzdorf, A., Morawietz, L. (2018a), Prädiktion der Lebensdauer von Traktionsbatteriesystemen für reale Nutzungsszenarien, in VDI Wissensforum GmbH (Hrsg.), Innovative Antriebe 2018: Der Ausblick auf die Fahrzeugantriebe für die kommenden Dekaden, VDI Berichte, VDI Verlag GmbH, Düsseldorf, S. 33-48.

Ufert, M., Bunzel, A. (2018b), Projektpräsentation: Forschungsprojekt EKE-ÖPNV, Dresden.

UITP (2017), ZeEUS eBus Report \#2: An updated overview of electric buses in Europe, Brüssel.

VDV (2016), Positionspapier - Elektromobilität weiter fördern, Köln.

Wackernagel, N. (2012), Ladezustandsbestimmung von Lithium-lonen-Zellen mit Hilfe eines Mikrocontrollers, Diplomarbeit, Technische Universität Dresden, Dresden, 2012.

Yan, Y. (2014), Thermische Modellierung einer Lithium-Ionen-Batterie, Diplomarbeit, Technische Universität Dresden, Dresden, 2014. 


\section{Abkürzungs- und Symbolverzeichnis}

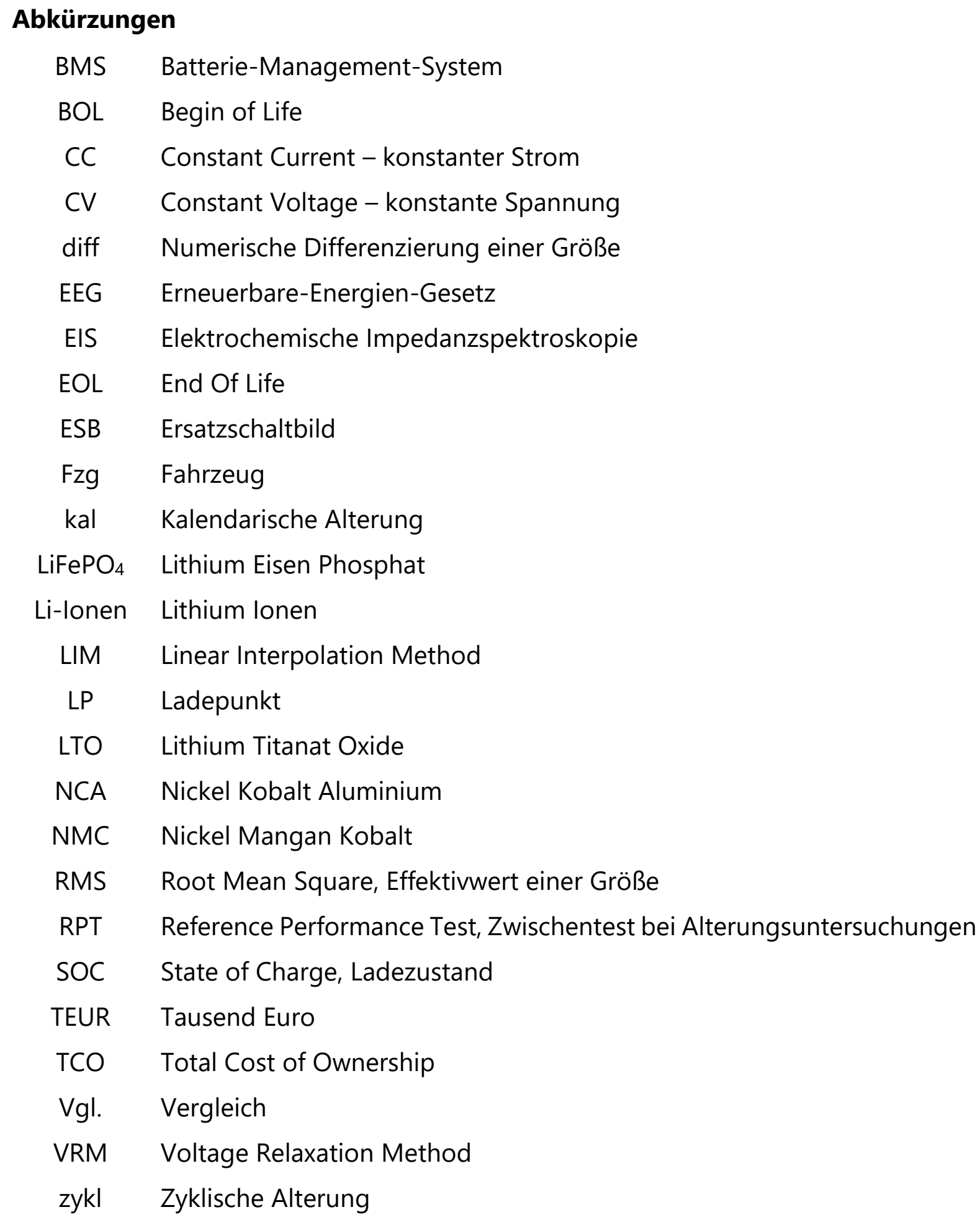




\section{Symbole und Formelzeichen}

C Kapazität

Co Ausgangskapazität

$\mathrm{C}_{\mathrm{N}} \quad$ Nennkapazität

$\mathrm{C}_{\text {act }} \quad$ Aktuelle Kapazität

k Spezifische Kosten

K Absolute Kosten

$\mathrm{m} \quad$ Anstieg einer linearen Funktion

n Anzahl

P Leistung

Pzwk Elektrische Leistung am DC-Zwischenkreis

Q Wärmestrom

Q Ladungsdurchsatz

$\mathrm{R}_{\mathrm{i}} \quad$ Ohmscher Innenwiderstand

$S$ Schädigungsfunktion

$\tau_{\text {mikro }} \quad$ Mikrozeitschritt

$\Delta \tau_{\text {mikro }} \quad$ Mikrozeitschrittweite

$\tau_{\text {makro }} \quad$ Makrozeitschritt

$\Delta \tau_{\text {makro }} \quad$ Makrozeitschrittweite

$\eta \quad$ Wirkungsgrad

T Temperatur

$\mathrm{T}_{\mathrm{amb}} \quad$ Umgebungstemperatur

$\mathrm{T}_{\text {Batt }} \quad$ Batterietemperatur

$\mathrm{t}$ Zeit

Z Kombinationen 


\section{Indizes}

\begin{tabular}{|c|c|}
\hline $\mathrm{chr}$ & Charge, Laden \\
\hline dis & Discharge, Entladen \\
\hline ges & Gesamt \\
\hline $\mathrm{i}, \mathrm{j}, \mathrm{k}, \mathrm{n}$ & Laufvariablen \\
\hline Invest & Investive Kosten \\
\hline kal & Kalendarisch \\
\hline $\mathrm{KI}$ & Klemme der Batterie bzw. Zelle \\
\hline konv & Konvektion \\
\hline LC & Life Cycle \\
\hline leit & Leitung \\
\hline $\lim$ & Limit, Begrenzung eines Wertes \\
\hline mess & Gemessener Wert einer Größe \\
\hline $\mathrm{N}$ & Nominalwert \\
\hline OCV & Open Circuit Voltage - Ruhespannung \\
\hline ref & Referenzwert einer Größe \\
\hline $\operatorname{sim}$ & Simulierter Wert einer Größe \\
\hline strahl & Strahlung \\
\hline Sys & System \\
\hline soll & Sollwert \\
\hline Temp & Temperatur \\
\hline th & Thermisch \\
\hline var & Variabel \\
\hline Verlust & Ohmsche Verluste \\
\hline Zelle & Auf eine Einzelzelle beziehend \\
\hline zykl & Zyklisch \\
\hline
\end{tabular}





\section{Abbildungsverzeichnis}

Abbildung 1-1: Roadmap zur Einführung von Elektrobussen, nach (Corneille, 2018)

Abbildung 2-1: Typische Fahrleistungen von Bussen im ÖPNV an Werktagen, oben: Solobusse (12 m), unten: Gelenkbusse (18 m), (Knote et al., 2017)...

Abbildung 2-2: Hauptbestandteile im System Elektrobus, nach (Rogge et al., 2015a)

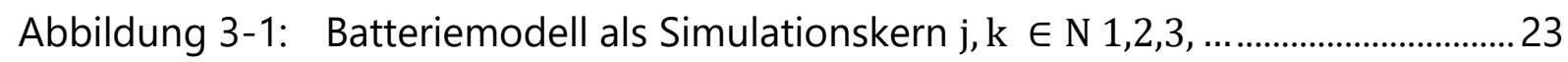

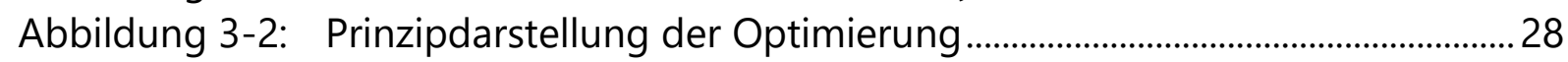

Abbildung 3-3: Globaler Programmablaufplan ........................................................................ 33

Abbildung 4-1: Funktionsprinzip einer Li-Ionen Zelle (Reif, 2012) .................................... 38

Abbildung 4-2: Übersicht der aktuell in Fahrzeugen eingesetzten Kathodenmaterialien (Bloomberg, 2018).

Abbildung 4-3: oben: Spannungsantwort einer Li-lonen Zelle auf einen

Stromsprung, (Jossen et al., 2006), unten: Zuordnung der

Spannungsfälle zum ESB..

Abbildung 4-4: Elektrisches Zell- und Batteriemodell..........................................................4 44

Abbildung 4-5: OCV-Kennlinie der LiFePO4 Zelle (Richter, 2017) ...................................45

Abbildung 4-6: Temperaturabhängige Entladekurven der LiFePO4 Zelle (A123 Systems, 2014)...

Abbildung 4-7: Kennfeld ohmscher Innenwiderstand Ri, Messdaten aus (Meubrink, 2016).

Abbildung 4-8: Beispiel einer Ladung im CC-CV-Verfahren (Jossen et al., 2006) ....... 49

Abbildung 4-9: Kennfelder Strombegrenzung der LiFePO4 Zelle (oben: Laden, unten: Entladen), Daten aus (A123 Systems, 2014)...

Abbildung 4-10: Gemessener Leistungs- und SOC-Verlauf für die Validierung des elektrischen Modells.

Abbildung 4-11: Vergleich Simulation und Messung - Batteriestrom IBatt....................52

Abbildung 4-12: Vergleich Simulation und Messung - Batteriestrom IBatt

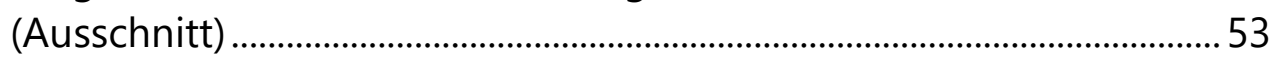

Abbildung 4-13: Vergleich Simulation und Messung - Klemmenspannung UKI ...........53

Abbildung 4-14: Vergleich Simulation und Messung - Klemmenspannung UKI (Ausschnitt)

Abbildung 4-15: Wärmeübertragungsprozesse: a) Wärmeleitung, b)

Wärmekonvektion, c) Wärmestrahlung (Kritzinger, 2019). .56 
Abbildung 4-16: ESB des thermischen Modells (Kritzinger, 2018a)

Abbildung 4-17: Temperatur bzw. Temperaturverteilung in konzentrierten, eindimensionalen und mehrdimensionalen Modellen, nach (Yan, 2014)

Abbildung 4-18: Rechteckiger Körper mit 6 Abstrahlflächen (Kritzinger, 2018b)

Abbildung 4-19: Summe der thermischen Teilwiderstände in Richtung x1, nach (Kritzinger, 2018a)

Abbildung 4-20: Thermische Teilwiderstände einer Einzelzelle in x-Richtung, nach (Kritzinger, 2018a)

Abbildung 4-21: Modellierung von Kühlstrategien (Kritzinger, 2018b)

Abbildung 4-22: Versuchszelle mit zwei Temperatursensoren, 1)

Oberflächentemperatur, 2) Umgebungstemperatur (Richter, 2017)

Abbildung 4-23: Validierungsergebnis thermisches Modell auf Zellebene

Abbildung 4-24: Validierungsergebnis thermisches Modell auf Batterieebene, Daten aus (Gatter, 2019)

Abbildung 4-25: Übersicht zur Alterung von Li-lonen Batterien

Abbildung 4-26: Messergebnisse Einflussfaktor Temperatur (zyklisch) (links), resultierende Schädigungsfunktionen (rechts).

Abbildung 4-27: Messergebnisse Einflussfaktor Temperatur (kalendarisch) (links), resultierende Schädigungsfunktionen (rechts).

Abbildung 4-28: Messergebnisse Einflussfaktor $\triangle \mathrm{SOC}$ (links), resultierende Schädigungsfunktionen (rechts)

Abbildung 4-29: Messergebnisse Einflussfaktor C-Rate (links), resultierende Schädigungsfunktionen (rechts)

Abbildung 4-30: Ladungsdurchsatz in Abhängigkeit der Versuchsdauer (Messreihe: C-Rate)

Abbildung 4-31: Messergebnisse Einflussfaktor SOC (kalendarisch) (links), resultierende Schädigungsfunktionen (rechts) 76

Abbildung 4-32: Workflow zur Berechnung dynamischer Stromprofile ............................ 81

Abbildung 4-33: Superposition von kalendarischer und zyklischer Alterung .................. 81

Abbildung 4-34: Zyklus A für die Validierung des Alterungsmodells .................................. 82

Abbildung 4-35: Zyklus B für die Validierung des Alterungsmodells .................................. 83

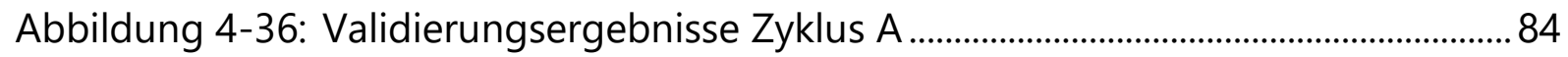

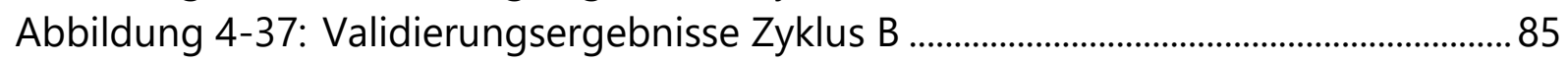

Abbildung 4-38: Übersicht eines konduktiven Ladepunkts (Büchner, 2015) .................... 87

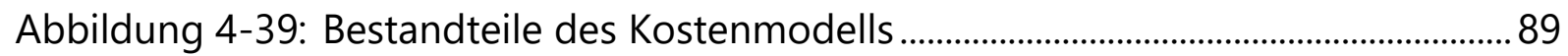

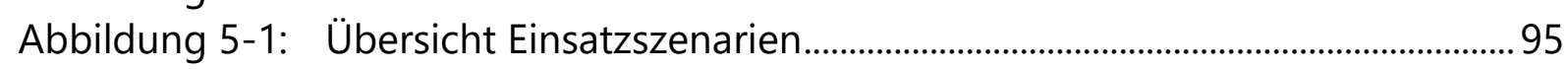

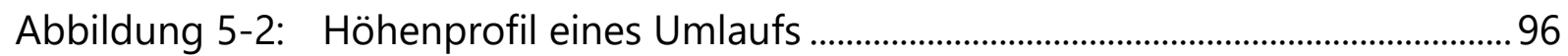

Abbildung 5-3: Simulierter Geschwindigkeitsverlauf eines Umlaufs ...............................97 
Abbildung 5-4: Simulierter elektrischer Leistungsbedarf am Zwischenkreis eines Umlaufs (Fahrzeug ohne Batteriegewicht)

Abbildung 5-5: Schemenhafte Darstellung der geometrischen Zellanordnung in einem Modul, nach (Kritzinger, 2018b)

Abbildung 5-6: SOC-Verlauf Basisszenario (10 parallele Stränge, 135 kWh, 200 $\mathrm{kW})$

Abbildung 5-7: Alterungsverläufe im Basisszenario .101

Abbildung 5-8: Numerisch differenzierte Alterungsverläufe im Basisszenario...........101

Abbildung 5-9: Verlauf der Alterung für 30 Tage bei einem Ruhetag pro Woche...102

Abbildung 5-10: Lebensdauer der Batterie in Abhängigkeit der jährlichen Betriebstage .103

Abbildung 5-11: SOC-Verlauf des Basisszenarios mit Ausfall einer Ladephase............105

Abbildung 5-12: SOC-Verlauf mit Ausfall einer Ladephase (162 kWh, 200 kW)..........105

Abbildung 5-13: Batterielebensdauer in Abhängigkeit der Anzahl an

Wochentagen mit Ausfallszenario.

Abbildung 5-14: Temperaturverläufe, links: Monatsmittelwerte für Deutschland (2001-2017), rechts: Tagesmittelwerte der Station DresdenKlotzsche (1991).

Abbildung 5-15: SOC-Verlauf der Basisszenario-Konfiguration bei $-12.6^{\circ} \mathrm{C}(10$ parallele Zellen, $135 \mathrm{kWh}$ )

Abbildung 5-16: SOC-Verlauf mit 34 parallelen Zellen ( $457 \mathrm{kWh}$ ) bei $-12.6^{\circ} \mathrm{C}$ 109

Abbildung 5-17: Batterielebensdauer bei verschiedenen Granularitätsstufen der Temperaturverteilung

Abbildung 5-18: Batterietemperaturverlauf des Basisszenarios, oben: ohne Batterieheizung, unten: mit Batterieheizung

Abbildung 5-19: Sensitivität der Ergebnisgenauigkeit der Alterungsberechnung in Abhängigkeit der Makrozeitschrittweite (Basisszenario).

Abbildung 5-20: Sensitivität der Lebensdauer in Abhängigkeit der Makrozeitschrittweite, (Variation der Zeitschrittweite und des SOC-Hubs)

Abbildung 5-21: Verlauf der Alterungsfaktoren über Makrozeitschritten

Abbildung 5-22: Änderung der Schädigungsfaktoren über Makrozeitschritten 118

Abbildung 5-23: Vergrößerung des SOC-Hubs durch Alterung (Basisszenario bis $\tau$ makro $=810 \mathrm{~d}$ )

Abbildung 5-24: Vergrößerung des SOC-Hubs durch Alterung und unzureichende Nachladung (Basisszenario von $\tau$ makro $=810 \mathrm{~d}$ bis $\tau$ makro $=1636 \mathrm{~d}$ )

Abbildung 5-25: Detailanalyse SOC-Hub: oben: Zunahme SOC-Hub über Makrozeitschritten, unten: differenzierter Verlauf des Schädigungsfaktors (Kapazität)...

Abbildung 5-26: Parametriertes Kostenmodell 
Abbildung 5-27: Optimierungsergebnisse (Basisszenario)

Abbildung 5-28: Kostenminimum für jede Ladeleistung mit zugehörigem

Energieinhalt der Batterie (Basisszenario)

Abbildung 5-29: Resultierender SOC-Verlauf der optimalen Konfiguration

(Basisszenario)

Abbildung 5-30: Resultierender SOC-Verlauf der Konfiguration 162 kWh, LP 1/1, 190 kW (Basisszenario)

Abbildung 5-31: Resultierender SOC-Verlauf der optimalen Konfiguration bei LP 1/0 (Basisszenario)

Abbildung 5-32: Resultierender SOC-Verlauf der optimalen Konfiguration bei LP 0/1 (Basisszenario)

Abbildung 5-33: Kostenbestandteile (LP 1/1, Basisszenario)

Abbildung 5-34: Optimierungsergebnisse (erweitertes Szenario)

Abbildung 5-35: Kostenminimum für jede Ladeleistung mit zugehörigem

Energieinhalt der Batterie (erweitertes Szenario)

Abbildung 5-36: Resultierender SOC-Verlauf der optimalen Konfiguration mit

Ausfallszenario (erweitertes Szenario).

Abbildung 5-37: Kostenbestandteile (LP 1/1, erweitertes Szenario).

Abbildung 5-38: Optimale Systemkonfiguration als Funktion des Fahrplantaktes ....135

Abbildung 5-39: Sensitivität der Kostenbestandteile 


\section{Tabellenverzeichnis}

Tabelle 2-1: Überschlägiger Reichweitenvergleich von Dieselbus und Elektrobus.......7

Tabelle 2-2: Übersicht der zwei etablierten Ladekonzepte.................................................. 10

Tabelle 2-3: $\quad$ Durchschnittliche Energieverbrauchskennzahlen (BMVI, 2016;

Schwarze, 2019) .................................................................................................... 12

Tabelle 2-4: Beispielrechnung für zwei verschiedene Auslegungsvarianten

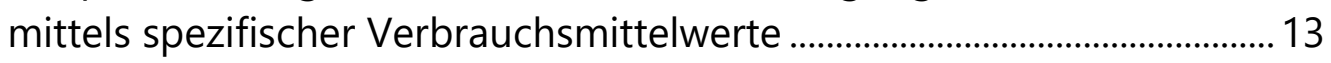

Tabelle 2-5: Übersicht Forschungsaktivitäten ........................................................................ 15

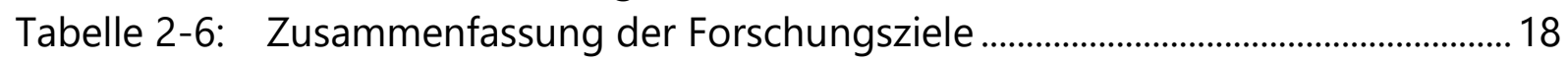

Tabelle 3-1: Optimierungsziele einzelner Systemparameter und

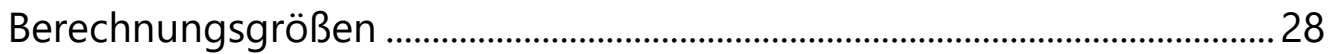

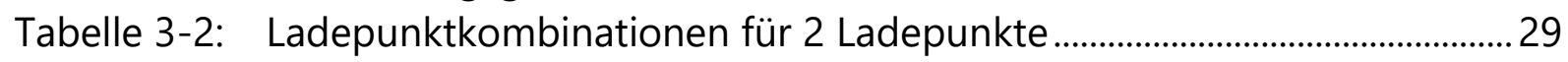

Tabelle 3-3: Beispiel des Wertebereichs und Schrittweiten der

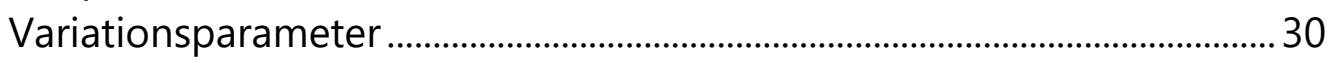

Tabelle 4-1: Übersicht Batteriemodellbildung und Validierung in Kapitel 4.1............ 35

Tabelle 4-2: Vergleich von Modellen für Batteriesysteme, nach (Jossen, 2016) .......... 37

Tabelle 4-3: Auszug aus dem Zelldatenblatt (A123 Systems, 2011, 2014) .....................41

Tabelle 4-4: Parameter des elektrischen ESB und ihre Abhängigkeiten ..........................44

Tabelle 4-5: Zusammenfassung der Validierungsergebnisse der elektrischen

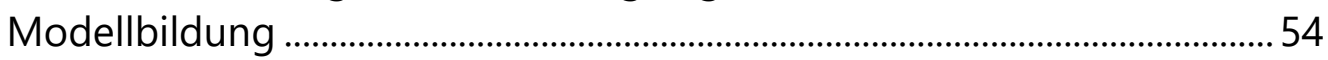

Tabelle 4-6: Übersicht konvektive Wärmeübergänge für verschiedene Kühlungsmechanismen (Kritzinger, 2018a).

Tabelle 4-7: Zusammenfassung der Validierungsergebnisse der thermischen Modellbildung (Zellebene) ....................................................................................... 65

Tabelle 4-8: Zusammenfassung der Validierungsergebnisse der thermischen

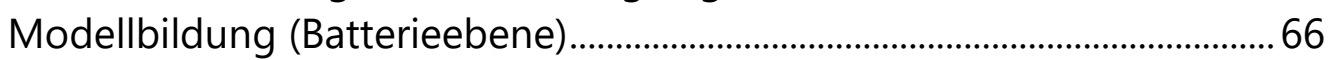

Tabelle 4-9: Messreihen zur Modellbildung (zyklische Alterung)....................................... 71

Tabelle 4-10: Messreihen zur Modellbildung (kalendarische Alterung) ............................. 71

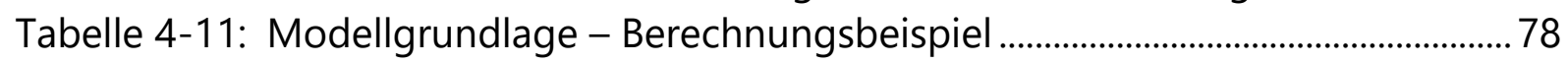

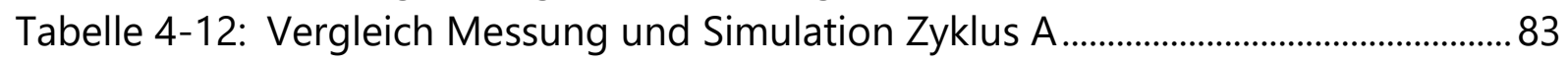

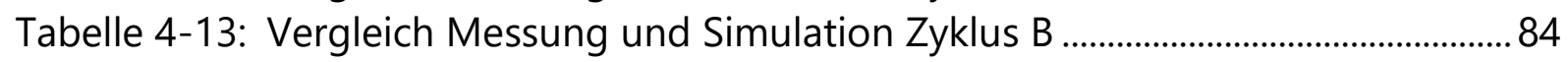

Tabelle 4-14: Wirkungsgrade verschiedener Ladetechnologien (BMVI, 2016) .............88

Tabelle 4-15: Kostenbestandteile der streckenseitigen Ladeinfrastruktur........................91

Tabelle 4-16: Kostenbestandteile und zugehörige Einheiten..............................................93

Tabelle 5-1: Spannungsbereich der LiFePO4 Zelle von A123 Systems............................98

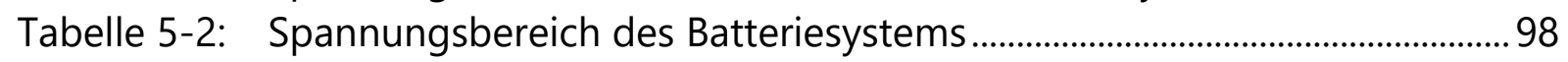


Tabelle 5-3: Betriebliche und technische Daten und Randbedingungen des Basisszenarios

Tabelle 5-4: beispielhaftes Betriebsprogramm.

Tabelle 5-5: Auswirkung der Ruhetage pro Jahr auf die Alterung der Batterie .........103

Tabelle 5-6: Auswirkung der Anzahl an Tagen mit Ausfallszenario auf die Lebensdauer der Batterie.

Tabelle 5-7: Auswirkung der Granularitätsstufen der Temperaturverteilung auf die Lebensdauer.

Tabelle 5-8: Sensitivität der Lebensdauer in Abhängigkeit der

Makrozeitschrittweite (Basisszenario)

Tabelle 5-9: Sensitivität der Lebensdauer (in Tagen) in Abhängigkeit der Makrozeitschrittweite (Variation der Zeitschrittweite und des SOCHubs)....

Tabelle 5-10: Grenzen der Variationsparameter

Tabelle 5-11: Zusammenfassung der Ergebnisse der optimalen Konfiguration (Basisszenario)

Tabelle 5-12: Optimale Systemkonfigurationen der Teilmengen LP 1/0 und LP $0 / 1$

Tabelle 5-13: Zusätzliche Randbedingungen zur Optimierungsrechnung des erweiterten Szenarios

Tabelle 5-14: Zusammenfassung der Ergebnisse der optimalen Konfiguration (erweitertes Szenario).

Tabelle 5-15: Vergleich der Optimierungsrechnungen

Tabelle 5-16: Vergleich der Optimierungsrechnungen mit weiteren

Batterieheizungssolltemperaturen

Tabelle 5-17: Optimierungspotential des erweiterten Szenarios. 134

Tabelle 5-18: Berechnungsbeispiel EEG-Umlage 138 


\section{Anhang}

\section{A.1 Optimierungsergebnis mit Batterieheizung (Sollwert: $15^{\circ} \mathrm{C}$ )}
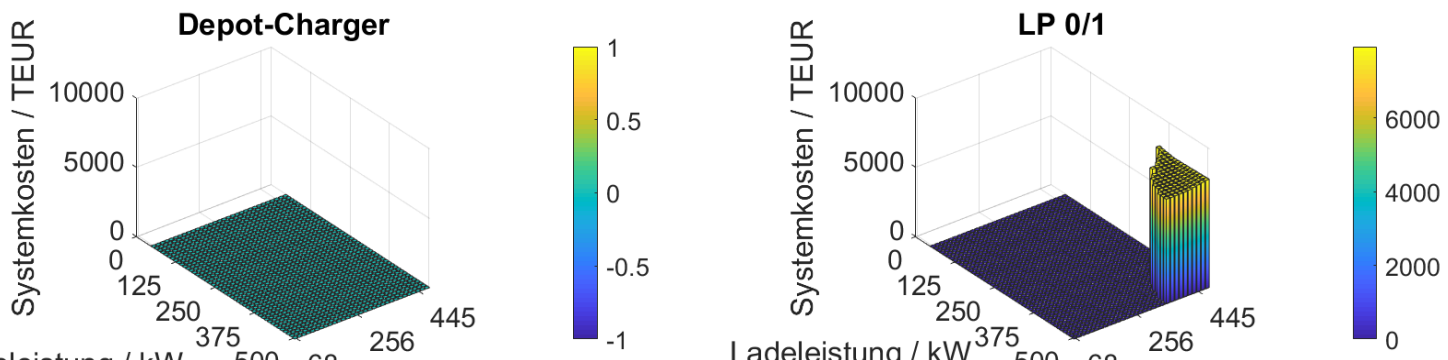

Ladeleistung / kW $500 \quad 68$ Energieinhalt Batterie / kWh Ladeleistung / kW 50068 Energieinhalt Batterie / kWh
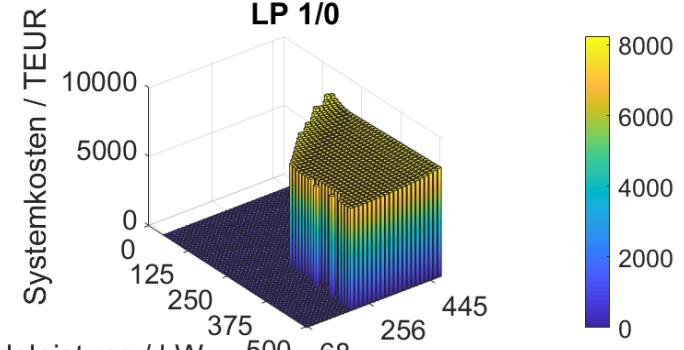

Ladeleistung / kW $\quad 500 \quad{ }^{68}$ Energieinhalt Batterie / kWh

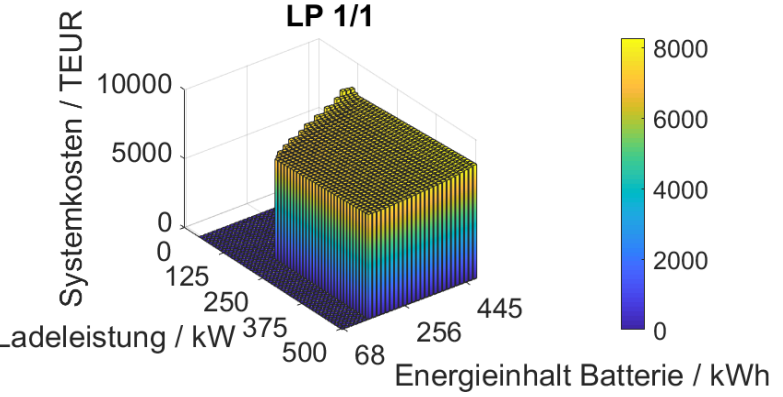

\section{A.2 Optimierungsergebnis mit Batterieheizung (Sollwert: $20^{\circ} \mathrm{C}$ )}
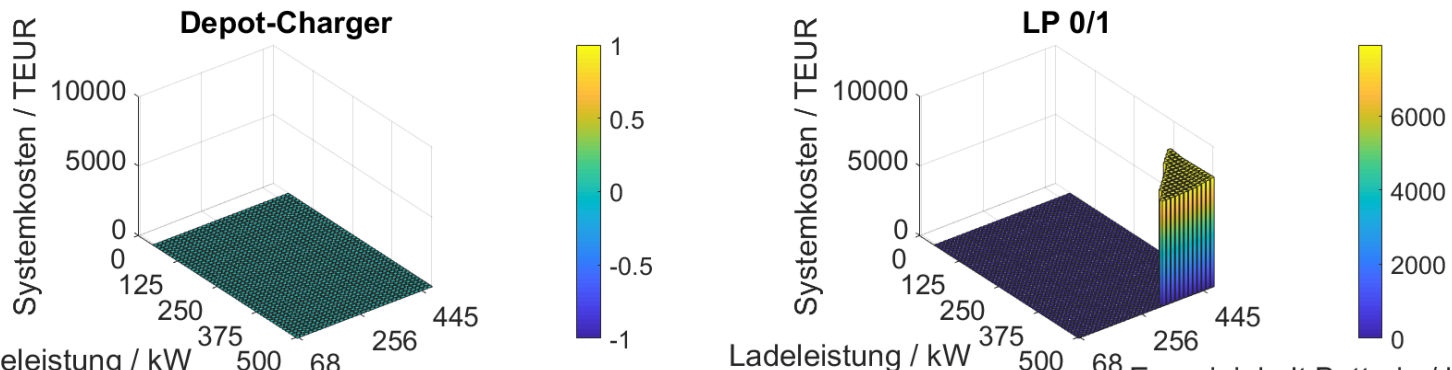

Ladeleistung / kW $500 \quad 68$ Energieinhalt Batterie / kWh

Ladeleistung / kW ${ }_{500}^{375} 68$ Energieinhalt Batterie / kWh

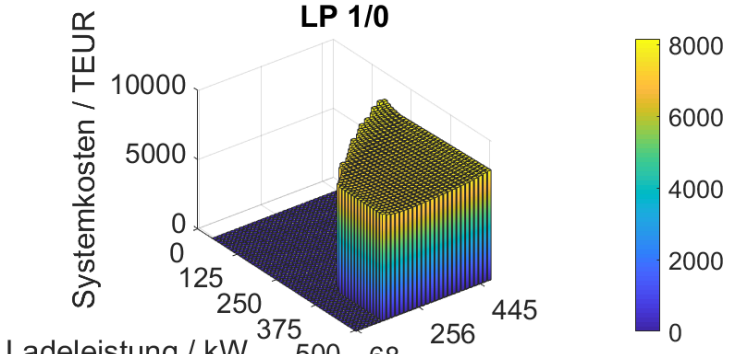

Ladeleistung / kW ${ }_{500}^{375} 68_{\text {Energieinhalt Batterie / kWh }}^{256}$

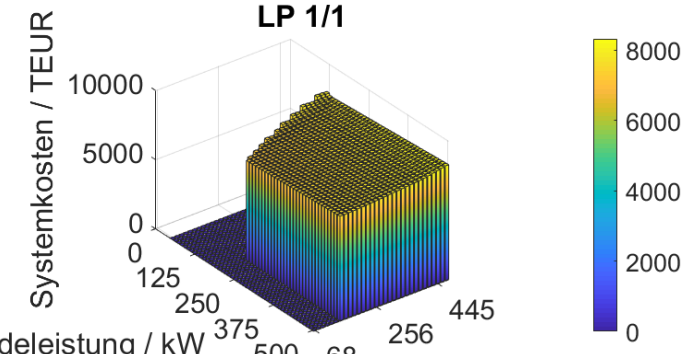

Ladeleistung / kW ${ }_{500}^{375} 68_{\text {Energieinhalt Batterie / kWh }}^{256}$ 


\section{B.1 Optimale Konfigurationen der Sensitivitätsanalyse}

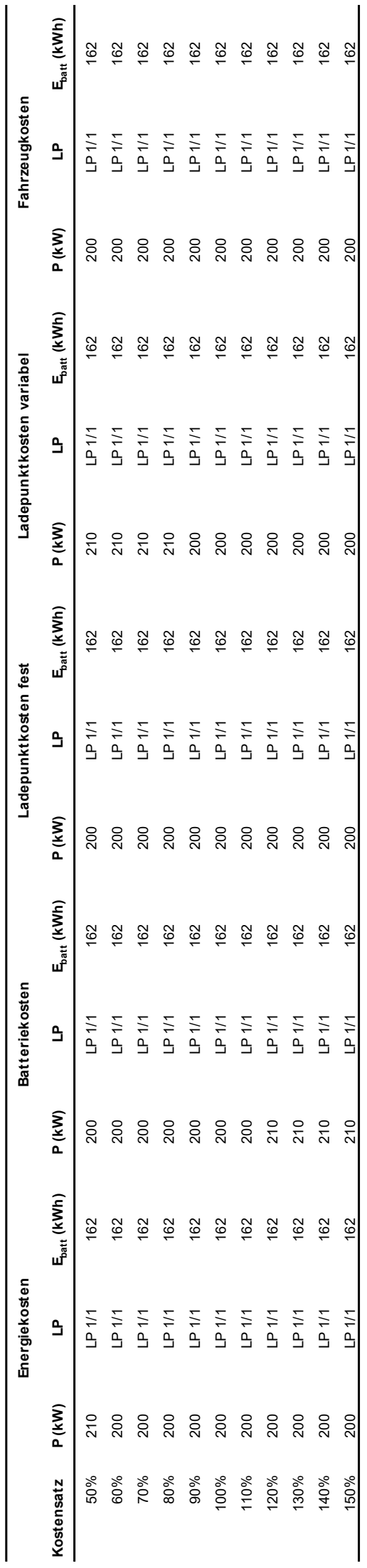


C.1 Stromprofil zur Validierung des thermischen Modells auf Zellebene

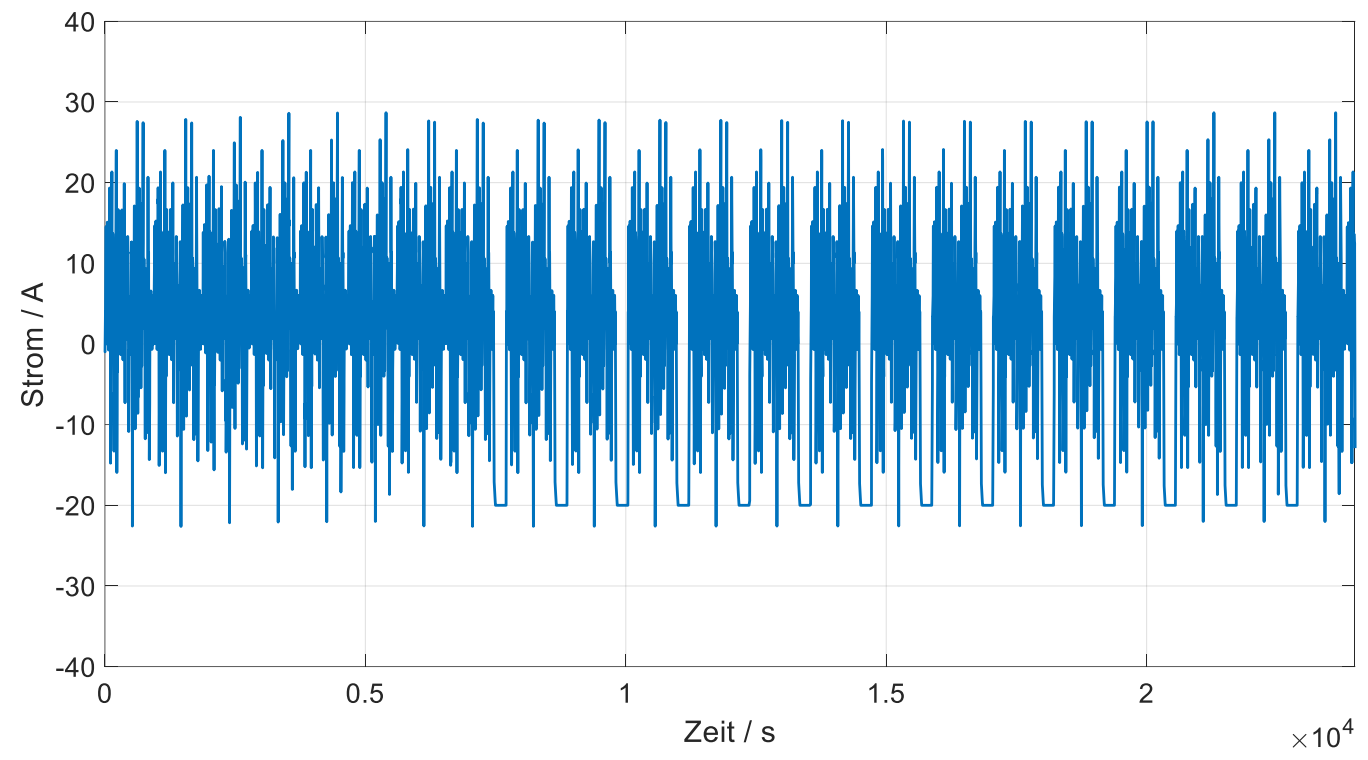

C.2 Stromprofil zur Validierung des thermischen Modells auf Batterieebene

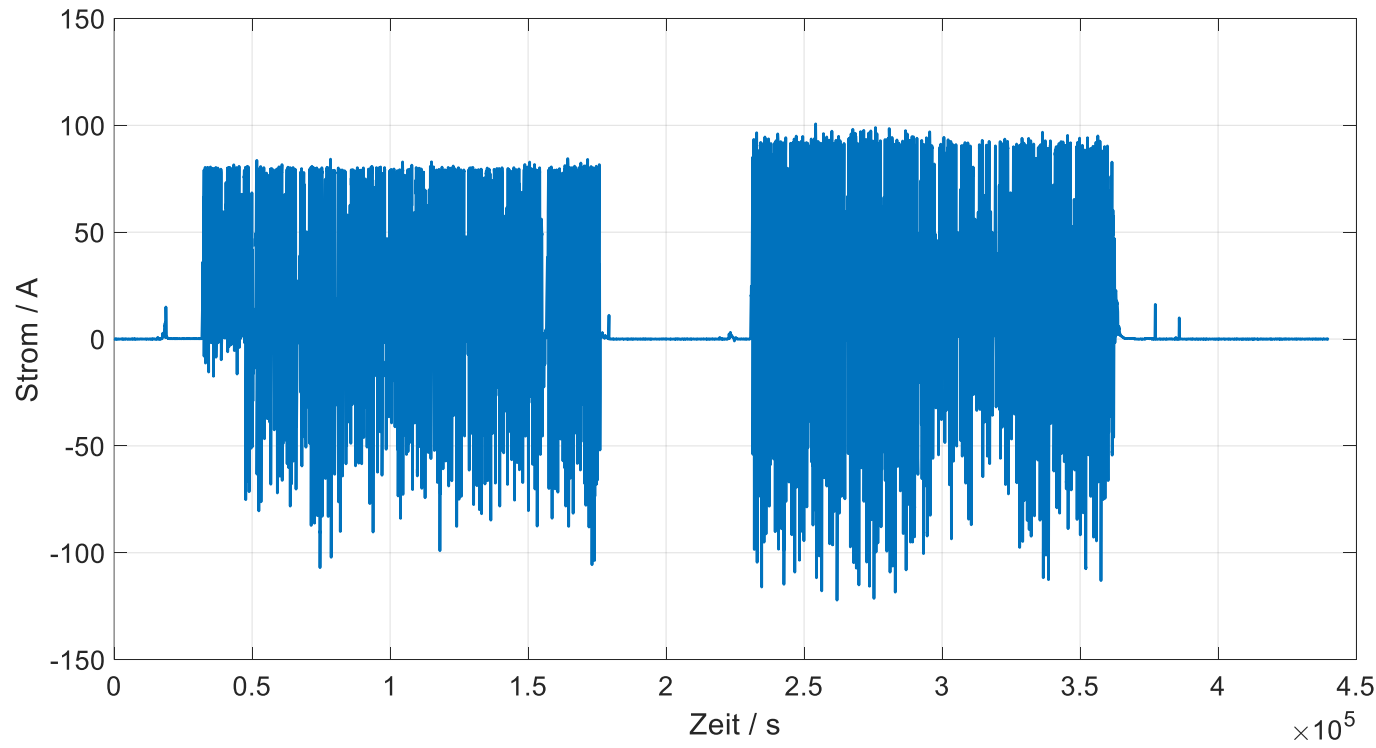




\section{D.1 Aufbau eines Batteriesystems}

Batteriesysteme sind oftmals mehrstufig aufgebaut. Die kleinste Einheit stellen dabei die Einzelzellen dar. Diese werden zu Batteriemodulen zusammengesetzt, wobei die Anzahl je nach Zellbauform variieren kann. Innerhalb des Moduls können die Zellen je nach Anwendungsfall seriell und/ oder parallel verschaltet werden. Mehrere Module bilden wiederum ein Batteriepack, welches dann in das Fahrzeug integriert wird. Die Anzahl der Module je Pack richtet sich vor allem nach dem verfügbaren Bauraum. Analog zu den Einzelzellen können die Module ebenfalls nahezu beliebig seriell und/oder parallel verschaltet werden. Zusätzlich beinhalten die Batteriepacks meist eine Steuerungselektronik in Form eines BMS sowie ein Sicherheits- und Kühlsystem. Die Zusammensetzung eines Batteriepacks aus Modulen und Einzelzellen ist am Beispiel einer prismatischen Zelle in Abbildung D.1-1 schematisch gezeigt (Kampker et al., 2018).

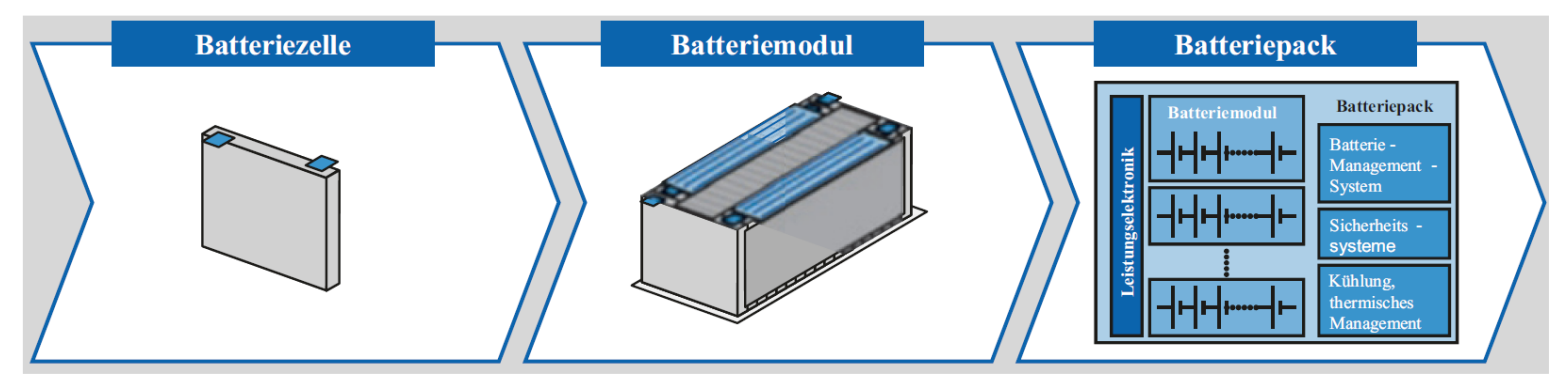

\section{Abbildung D.1-1: Zusammensetzung eines Batteriesystems aus Einzelzellen und Modulen (Kampker et al., 2018)}

Bei Anwendung in Elektrobussen sind oftmals mehrere Batteriepacks pro Fahrzeug erforderlich, um die Anforderungen an Reichweite, Leistungsfähigkeit und Lebensdauer erfüllen zu können. Ein Beispiel eines Batteriepacks für Busanwendung ist in Abbildung D.1-2 gegeben.

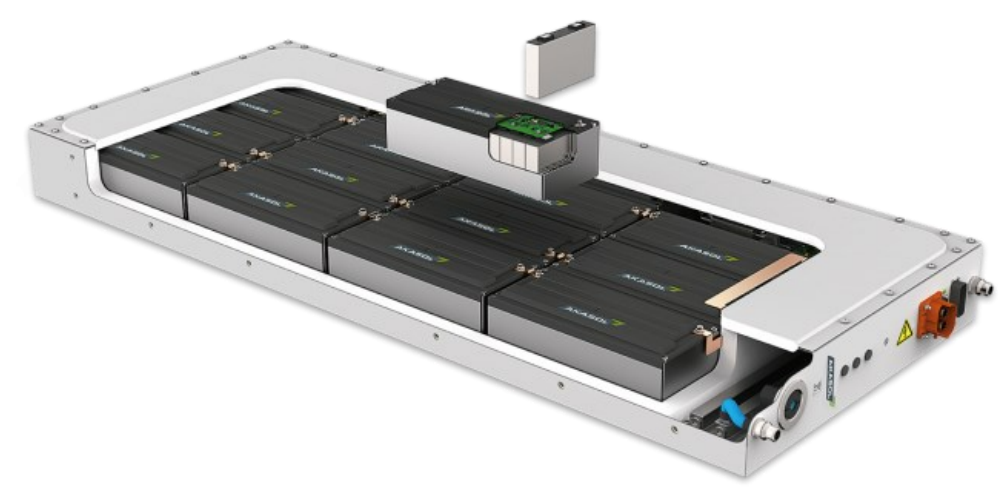

Abbildung D.1-2: praktische Umsetzung eines Batteriepacks (Akasol AG, 2019) 


\section{E.1 Parameter des RC-Glieds aus EIS-Messung und Parameter Fitting}

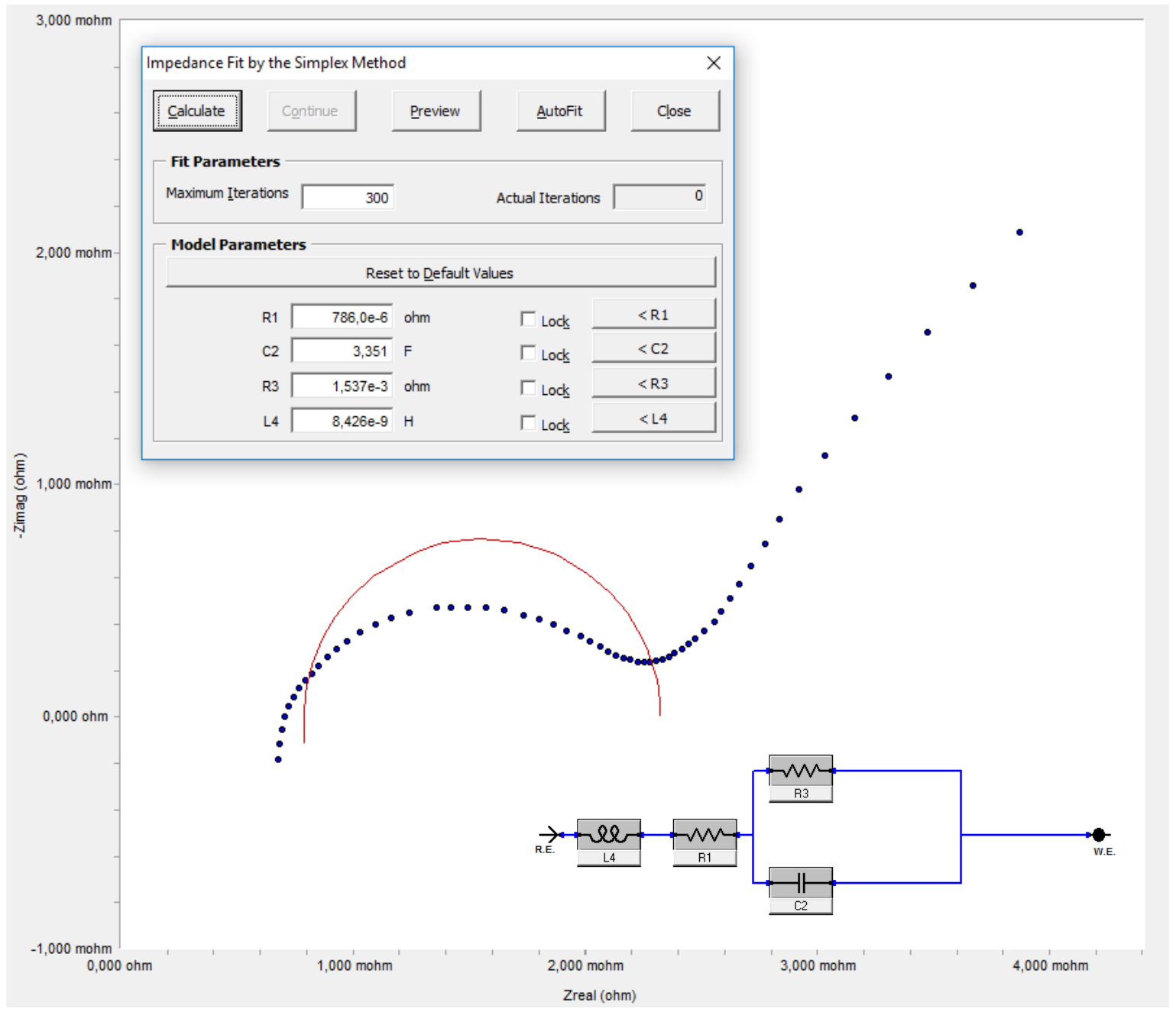

